The neural and behavioral bases of

Active touch and tactile working memory

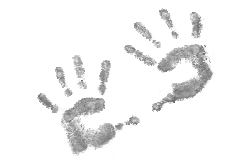

Amanda L. Kaas 
De foto op de omslag is een bewerking van "the weaver" uit de serie "Life in Sonora, Mexico" van L. Gene Richardson, Plano, Texas, USA. Copyright (C) 1999

J.L. Richardson, gereproduceerd met toestemming.

Gedrukt door Datawyse

(C) copyright A.L. Kaas, Frankfurt 2006

Universitaire Pers Maastricht

ISBN 10: 90-5278-573-2

ISBN 13: $978-90-5278-573-8$ 


\title{
The neural and behavioral bases of \\ Active touch and tactile working memory
}

\author{
De neurale en gedragsmatige basis van \\ actieve tast en tactiel werkgeheugen.
}

\section{PROEFSCHRIFT}

ter verkrijging van de graad van doctor aan de Universiteit Maastricht, op gezag van de Rector Magnificus, Prof mr. G.P.M.F. Mols volgens het besluit van het College van Decanen, in het openbaar te verdedigen op vrijdag 20 oktober om 14.00 uur

door

Amanda Leonie Kaas

Geboren op 9 oktober 1975 te Alkmaar

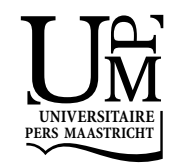




\section{Promotor:}

Prof. dr. R. Goebel

\section{Copromotor:}

Dr. H.I. van Mier

\section{Beoordelingscommissie:}

Prof. dr. G.J. Kok (voorzitter)

Dr. E. Formisano

Prof. dr. A.M.L. Kappers (Universiteit Utrecht)

Prof. dr. R.J. Seitz ～(Heinrich Heine Universität, Düsseldorf, Duitsland)

Dr. P. de Weerd

Het in dit proefschrift beschreven onderzoek werd mede mogelijk gemaakt door een subsidie van de Nederlandse Organisatie voor Wetenschappelijk onderzoek (NWO, no. 410-20-205). 
Voor Nellie, Nico en Annelies 



\section{CONTENTS}

GENERAL INTRODUCTION 11

HAPTIC SPATIAL MATCHING 31

THE EFFECT OF VISUO-HAPTIC CONGRUENCY ON HAPTIC SPATIAL MATCHING 51

THE EFFECT OF BODY POSTURE ON HAPTIC SPATIAL MATCHING 69

THE NEURAL CORRELATES OF HAPTIC WORKING MEMORY FOR ORIENTATION 83

THE NEURAL CORRELATES OF HAPTIC WORKING MEMORY FOR TEXTURE 111

SUMMARY AND CONCLUSIONS 135

SAMENVATTING EN CONCLUSIES 140

EPILOOG 146

CURriculum VITAE 150

APPENDIX 151 



\title{
CHAPTER 1
}

\author{
General Introduction
}


10 | Chapter 1 


\section{General Introduction}

When asked to name the human senses, most people will start with vision and hearing, then go to smell and taste, and finally mention touch. The order of enumeration and omissions in the list might to some extent reflect the dominance each of these sensory modalities has in subjective perception. Only when we are unable to use the faculties of vision and audition, e.g. in a noisy, dark environment or in case of an illness or injury, we become aware of the rich world of information to be discovered by the sense of taste, smell and touch.

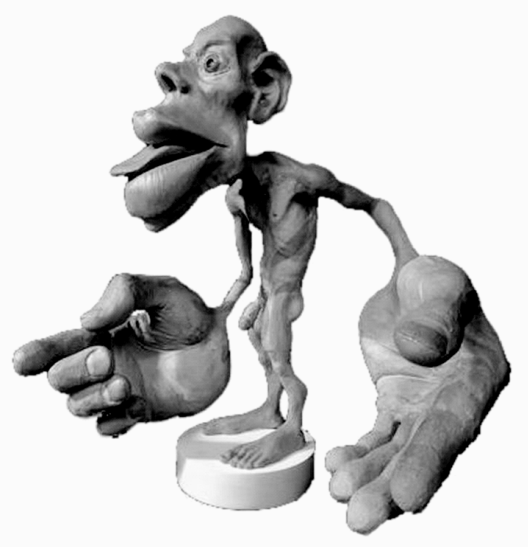

Figure 1. A model of the somatosensory homunculus. The relative size of the body parts indicates the relative density of cutaneous (skin) receptors in these body parts, and the portion of the primary somatosensory cortex dedicated to the processing of signals originating from these receptors. (From the collection of the Natural History Museum, London, U.K.)

Apart from vision, the sense of touch is the only other sense which can give geometrical object information, and it is of crucial importance in skilled object manipulation, ranging from simply lifting a cup to performing complex surgery. Object manipulation skills started to develop from the moment that our prehuman ancestors began walking 
upright, marking a turning point in the evolution of our species (e.g. Donald, 1997). Our advances in the use of tools made it possible to cooperatively mold the world around us according to our needs and liking, and almost literally paved the way for modern society. Moreover, Berkeley and other empiricist philosophers from the eighteenth century even suggested that knowledge of the properties of our environment can only be acquired through active interaction by means of the somatosensory system (e.g. Zimbardo et al., 1995). This process might be reflected in the typical explorative behavior of young infants, grasping all objects within reach. Although Berkeley's claim is at odds with the important role of maturation and observation learning in development, it is evident that hands-on training provides us with important cognitive constraints and insights which are hard to acquire otherwise.

However, just as we are often unconscious of the abounding diversity of information provided by the tactile sense and our hands in particular, the tactile sense only plays a marginal role in cognitive research and is often neglected in theories and models describing human cognition. Nevertheless, a considerable portion of the cortical surface is dedicated to the processing of somatosensory (i.e. body sense) information, and information from the hands in particular (see figure 1).

The goal of the present thesis is to illuminate the spatial reference frames involved in tactile object manipulation, as well as the brain areas underlying short-term maintenance of object information obtained through active touch. To this end, a number of behavioral and brain imaging experiments were carried out, the results of which will be presented and discussed in the next chapters. This introductory chapter will first present background information on the methods and theory relevant to this research goal, and then give an outline of the content and specific aims of the following chapters.

\section{From experimental psychology to cognitive neuroscience}

The research described in this thesis applies the tried and tested method of psychophysics, investigating the laws of the mind by measuring behavioral responses in a controlled experimental set up. This method has a history of more than a century. In addition, the final two chapters used the relatively new technique of functional magnetic resonance imaging (fMRI), by which changes in brain activity in response to cognitive tasks can be measured and localized. This technique has changed the face of cognitive psychology in the last decade. Just imagine: in 1995, our colossal "Introduction to Psychology" book contained just a small paragraph on imaging. Today, a faculty of Psychology seems incomplete when it does not have access to a magnetic resonance scanner...

In stark contrast, the very first experimental psychologists working in Wilhelm Wundt's laboratory in Leipzig, Germany, could do with no more than a paper and a pen to investigate human cognition. In those last decades of the nineteenth century, the main research method was introspection, a method in which highly trained participants were asked to report the contents of their consciousness under carefully controlled conditions. The study of the mind in carefully controlled experimental set-up was a great leap forward from the speculations on the workings of the mind that some important philosophers like Kant, Descartes and Berkeley in the preceding centuries had occasionally engaged in. However, it turned out that although the experimental set-up was rigid, the contents of the mind were very elusive and tended to reflect just 
the theory that the particular researcher who recorded them was hoping to put forward. At the beginning of the twentieth century, the advent of Behaviorism in the United States swept all concerns with consciousness and mental representation aside, stating that psychology should not bother with concepts that cannot be objectively defined, directly observed and quantified. Using objective operationalizations of the concepts under investigation, the behaviorists primarily investigated animal learning and motivation using reaction time and accuracy as their dependent measures. Only after a few decades, some brave scientists calling themselves cognitive psychologists again endeavored to extend their investigations into the "black box", the erratic domain of mental representations; hoping to find a theory of internal processes which would aid the understanding of human behavior. In response to developments in information theory, artificial intelligence and linguistic, boomed by the extraordinary demands posed by World War II, cognitive psychology attempted to decompose cognition into a set of information processing steps. The resulting flow-diagrams, in the best tradition of early computer programming, did not refer to any brain processes or locations, nor did it consider possible neural representation of the information.

In the mean time, medical science had rapidly advanced, establishing links between certain brain areas and specific cognitive functions. In the late nineteenth century, illustrious men like Broca and Wernicke described the remarkable symptoms displayed by patients with particular brain lesions. Around the turn of the century, Gall introduced phrenology, relating the shape of the skull to certain cognitive faculties. In the middle of the $20^{\text {th }}$ century, the Canadian neurosurgeon Wilder Penfield functionally mapped the cerebral cortex by electrical stimulation, to be able to confine surgery to non-vital brain areas as much as possible.

However, study of the brain only seriously started to attract the interest of experimental psychologists with the development of functional magnetic resonance imaging (fMRI). Magnetic Resonance Imaging was first proposed by Nobel laureate Prof. Lauterbur in 1973, and the first anatomical human brain images were obtained in 1980. The first so-called functional images of human brain activity were observed by Prof. Ogawa in 1990. The fascinating possibility to obtain images of the working human brain, without harming it, promoted a rapid expansion of the cognitive neurosciences, a scientific domain at the cross roads between psychology, medicine, and biomedical physics.

\section{Functional magnetic resonance imaging}

Magnetic resonance Imaging (MRI) is non-invasive imaging technique which can provide both high-resolution anatomical information as well as functional data of the human brain. The functional "image" consists of a series of scans with a spatial resolution in the order of millimetres, acquired repeatedly over time. Unlike other functional imaging techniques, fMRI does not require the injection of a contrast fluid, but instead measures changes in the blood oxygenation level dependent (BOLD) signal intensity. To obtain an MR image, the subject has to lie still inside a cylinder and is exposed to a high magnetic field (ranging from 0.5 to 7 Tesla), inducing a slight magnetization of body tissues, due to the alignment of nuclear spins of hydrogen atoms. A 3-D image is constructed slice by slice, beaming a high frequency electromagnetic (radio frequency, RF) pulse through the tissue, which causes energy to be absorbed by hydrogen nuclei 
(protons). This perturbs the spin alignment and, consequently, the magnetization. After turning off the RF pulse, magnetization returns to the equilibrium state, inducing a signal in the MR receiver coils. The type of molecule the hydrogen atom forms part of, and the kind of molecules surrounding it, determine the relaxation time (return to baseline) of its spin and the strength of the emitted signal. The fMRI BOLD response derives from the fact that deoxygenated hemoglobin contains free iron ions which reduce signal strength. When presynaptic neuronal activity in a brain area increases, more oxygen is used from the blood. In response to this, blood flow and the concentration of oxygenated hemoglobin in that neuronal area are augmented, causing a signal increase due to the decreased ratio of deoxy- and oxyhemoglobin. This signal increase, the hemodynamic response, reaches its peak a few seconds after the change in neuronal activity.

Although the ideal of cognitive neuroscience might be to relate the process descriptions of cognitive psychology one-to-one to activity changes in specific brain areas, this is not entirely realistic. In the first place, it would require that the brain is entirely organized in functional modules, which scale would have to correspond to the scale of the available imaging techniques. Although this might be true to some extent, it is very likely that higher cognitive functions involve distributed processes in the brain. A second issue which comes up is whether the functional modules and labels proposed by cognitive psychology correspond to the functional modules of the brain, to the extent that these can be revealed by fMRI. In many occasions, functional imaging has revealed that this is not the case, showing that the behavioural measures used by cognitive psychology afford a different level of description than the brain activity measures in cognitive neuroscience. Therefore, effective experimental designs in cognitive psychology might not always result in interesting fMRI designs. In the third place, there are some practical constraints in fMRI which have to be taken into account. The fact that fMRI requires a strong magnetic field constrains the type of stimuli to nonmagnetic materials. In addition, the narrow bore of the magnet limits lateral motion of the arms, and the head has to remain stable throughout the experiment. Finally, hand and arm movements which are too abrupt or too big in amplitude can cause signal artefacts when propagating to the shoulders, neck and head, or when disturbing the homogeneity of the magnetic field.

Hence, although fMRI provides a unique chance to peek into the "black box" of cognition, the characteristics of this method also changes the type of tasks that can be used, especially in studying active touch. Finding creative fMRI paradigms which can go beyond the domain of perception and cognition to also incorporate the neural bases of action will remain a continuing challenge. Even with fMRI experiments becoming increasingly sophisticated, behavioural and functional data will remain complementary. Both may provide important insights into cognition, especially when studying active touch. Using well-tested paradigms from psychophysics both in and outside the scanner, the current thesis attempts to expand the present knowledge of the anatomy of the somatosensory system and the properties of active touch into the realm of the neural substrates governing human haptic working memory. 


\section{Functional anatomy of the somatosensory system}

The somatosensory system is made up of two different components. One of these is exteroceptive, i.e. specialized to perceive external events, like the texture and movement of objects along the skin. The other component is proprioceptive, i.e. tuned to internal experiences, allowing us to feel the static position and movement of fingers and limbs. More specifically, the term kinesthesia is used to indicate the sense of movement arising from receptors in muscles tendons and joints.

The information obtained by touch and proprioception enters the central nervous system through the spinal dorsal root ganglion cells and the dorsal column. After a synapse in the cuneate nucleus of the medulla, the somatosensory pathway crosses to the contralateral side, and proceeds through the medial lemniscus to the ventral posterio lateral nucleus of the thalamus. There, it synapses on neurons projecting to the primary somatosensory cortex (S1), Brodmann areas (BA) 1, 2 and 3, located in the postcentral gyrus and adjacent areas. Basic processing takes place in BA3, subdivided into part $a$ (deep proprioceptive receptors) and part $b$ (cutaneous inputs). More complex processing occurs in BA1. In BA2, cutaneous and proprioceptive information are combined. The secondary somatosensory cortex (S2), located ventrally from SI, receives its projections principally from SI. Areas BA $3 \mathrm{~b}$ and 1 were shown to contain a complete representation of the body surface in primates (e.g. Kaas et al., 1979). They are characterized by a somatotopic organization: neighboring neurons respond to neighboring parts of the skin (Gardner and Kandel, 2000).

\section{Extracting object features by active touch}

The role of kinesthetic information in touch points to an important characteristic of the tactile sense: it closely links exploratory movements and sensory impressions. In other words, identifying an object using the tactile sense requires actively touching it. Different movement sequences allow us to extract distinct features, such as shape or surface texture (Lederman and Klatzky, 1987; figure 2). Hence, active finger movements are an essential part of tactile perception.

Active tactile perception of the three dimensional world is referred to as haptics, and is considered crucial for skillfull interaction with and manipulation of objects in our environment $($ haptic $=$ relating to the sense of touch, tactile; from the Greek haptesthai = to grasp, touch). Haptic object information exceeds visual information in richness; apart from shape and size, it also gives information on material properties such as surface roughness, compliance and thermal conductance (Lederman and Klatzky, 2004). People are remarkably skilled at recognizing common objects using only the sense of touch. In a study done by Klatzky and Lederman (1985), participants needed only two to three seconds to identify almost $100 \%$ of all the objects that they haptically explored. Moreover, when the hand is guided to an informative part of an object, classification is possible within $200 \mathrm{~ms}$ (Klatzky and Lederman, 1995).

The type of information obtained through haptics can be classified based on the type of object characteristics it reveals. Substance or material properties are at the one extreme of this classification scheme, object structure or geometry is at the other end. In contrast, we can classify haptic sensory impressions based on the type of processing required. Logically, tactile impressions can use an intensive (i.e. scalar amplitude cod- 
ing), spatial, temporal or modal coding (Lederman and Klatzky, 1997). Modal coding relates to the type of physical energy, thermal or mechanical. Intensive coding refers to representations in terms of a scalar quantity without reference to a planar spatial location or orientation, which is a defining feature of spatial representations. The computations in intensive processing are a prerequisite for spatial processing; therefore, properties that are intensively coded become available before those that require spatial processing. Lederman and Klatzky (1997) inferred that material properties (e.g. roughness) were coded intensively, while structural or geometrical properties used either intensive (e.g. abrupt surface discontinuities) or spatial processing (e.g. relative orientation), or a combination of both (e.g. curvature). In line with Treisman's feature integration theory, Sathian and Burton (1991) concluded that roughness might be a tactile primitive, even though it is represented at a cortical and not at a peripheral neuronal level. They based this conclusion on their finding that attentional cues did not affect the accuracy by which participants could detect a roughness change in the presence of constant-roughness distractors.

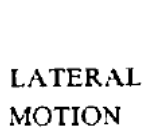
MOTION
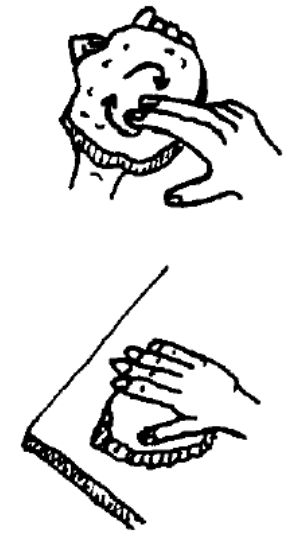

STATIC CONTACT

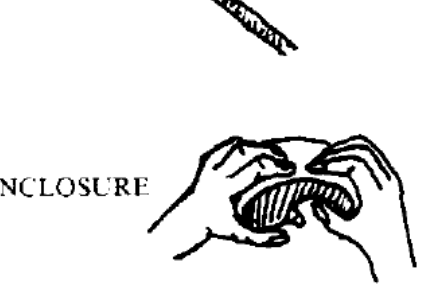

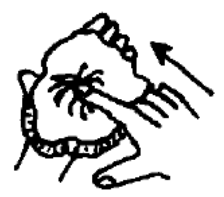
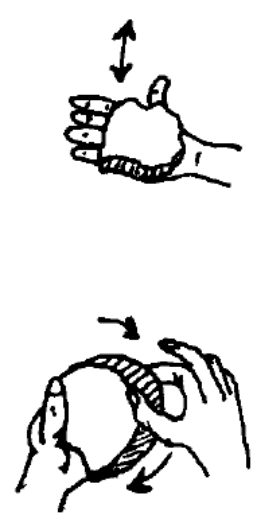

PRESSURE

UNSUPPORTED HOLDING

CONTOUR FOLLOWING

Figure 2. Lateral motion is used to extract texture, pressure for hardness, static contact for temperature, unsupported holding for weight, enclosure for global shape or volume, contour following for global or exact shape. Modified from Lederman and Klatzky (1987). 
There is evidence for the involvement of different brain areas for tactile processing of micro spatial (e.g. roughness) versus macro spatial (e.g. orientation) object features in non-human primates. When subjected to a lesion of Brodmann area 1, non-human primates showed impoverished discrimination learning of micro spatial material features such as roughness, while monkeys with a lesion in area 2 showed retarded discrimination learning of macro spatial features related to object structure, involving surface curvature and angles (squares versus diamonds; Randolph and Semmes, 1974). In humans, there is some evidence for parallel processing of micro spatial and macro spatial features (Roland et al., 1998; Bohlhalter et al., 2002; Merabet et al., 2004; Zhang et al., 2005). Bohlhalter et al. (2002) describe a case study of two patients with lesions to the primary and secondary somatosensory cortex, but with preserved ability to detect light touch, vibration and finger position. Although both patients were impaired in assessing micro spatial roughness differences of tactile gratings, the patient with lesions to the left supramarginal gyrus and left superior parietal lobule also displayed difficulties in tactile macro spatial discrimination of length. Imaging studies in healthy participants revealed that right hand micro spatial discriminations (roughness) specifically activated left secondary somatosensory (Roland et al., 1998) and right angular gyrus (Zhang et al., 2005), while discrimination of macro spatial object features recruited the left anterior intraparietal sulcus (Roland et al., 1998; Bodegård et al., 2001; Zhang et al., 2005) and left anterior supramarginal gyrus (Bodegård et al., 2001).

Some studies operationalized micro and macro spatial processing by using different stimuli (Roland et al., 1998; Bohlhalter et al., 2002), while others varied task instruction (Sathian et al., 1997; Zangaladze et al. 1999; Merabet et al., 2004; Zhang et al., 2005) Comparison of the studies reveals that some used active touch ( Roland et al., 1998; Bohlhalter et al., 2002; Merabet et al., 2004), while others used passive touch (Sathian et al., 1997; Zangaladze et al., 1999; Merabet et al., 2004; Zhang et al., 2005). More importantly, it exposes the use of contradictory task instructions and spatial scales. The distinction made by Bohlhalter et al. and Roland et al. is probably the clearest: they assessed micro spatial processing by asking participants to use active touch to discriminate the roughness of surfaces with wave length variations around $1 \mathrm{~mm}$. Macro spatial processing was induced by judgments of the oblongness of ellipsoids or parallelepipeds with volumes in the order of $11 \mathrm{~cm}^{3}$. However, in these studies, stimulus differences might be a confound when comparing macro versus micro spatial discrimination. Avoiding this confound, Merabet et al. (2004) used identical Braille like stimuli with dot spacings between 1 and $8 \mathrm{~mm}$, asking for a judgment of either roughness (micro spatial) or distance (macro spatial). Also ruling out potential differences in finger movements, Zhang et al. (2004) Zangaladze et al. (1999) and Stoesz et al. (2003) passively applied their tactile stimuli with gap distances up to $3 \mathrm{~mm}$, and considered orientation and grating distance to be independent macro and micro spatial features (this might hold only for passively applied gratings). Hence, whereas passive discriminations of distances up to $3 \mathrm{~mm}$ have been considered microspatial in some studies, active tactile judgments of dot spacings between 1 and $8 \mathrm{~mm}$ were considered macro spatial, and roughness judgments on the same stimuli micro spatial, by another (Merabet et al., 2004). Below $3 \mathrm{~mm}$, perceived roughness and groove width are linearly related. Given the size of cutaneous receptive fields in the finger tip, tactile distances below $3 \mathrm{~mm}$ might still be spatially resolved, but might additionally evoke intensive 
processing, in which spatial features are not singled out but induce a scalar impression of roughness. However, since active touch might increase tactile resolution, the role of spatially resolved processing for distances between 1 and $3 \mathrm{~mm}$ might be stronger in the study by Merabet et al. (2004). On the other hand, intensive processing might be more dominant in the passive grating distance judgments in the experiments of Zhang et al. (2004), Zangaladze et al. (1999) and Stoesz et al. (2003).

\section{Touch and vision}

Discrimination of macro spatial characteristics has been accompanied by reports of visual imagery, and areas belonging to the visual cortical pathway have been reported in imaging studies using macro spatial tasks, involving objects, shapes and grating orientations in sighted participants (Sathian et al., 1997; Zangaladze et al., 1999; Amedi et al., 2001, 2002; James et al., 2002; Pietrini et al., 2004) and objects and Braille in congenitally blind participants (Sadato et al., 1996; Pietrini et al., 2004). The fact that similar results were found for people who have no visual experience, suggest that the activation in supposedly visual areas of particularly the lateral occipito-temporal complex might not be due to visual imagery, but instead reflect modality independent macro spatial neural processing for object discrimination (Amedi et al., 2001). Using grating orientations, Zangaladze et al. (1999) found that transcranial magnetic stimulation (TMS) over the visual cortex interfered with tactile discrimination. In another TMS study using Braille like stimuli, Merabet et al. (2004) found a double dissociation between micro spatial (roughness) and macro spatial (distance) processing of raised dot patterns. Low frequency repetitive (r)TMS over the occipital cortex of sighted and congenitally blind participants impaired tactile judgments on dot pattern spacing, while roughness judgments performed on the same tactile dot patterns remained unaffected. The reverse was true for TMS over primary somatosensory regions, which impaired judgments of roughness but not distance.

Based on their results, Merabet et al. (2004) concluded that extrastriate visual areas are functionally involved in macro spatial tasks but not in micro spatial tasks in both blind and seeing participants. This suggests that the likelihood for visual areas to be recruited for a specific task might be modulated by the effectiveness of a visual strategy for the task at hand. This would be in accordance with the modality appropriateness hypothesis, originally proposed by Welch and Warren in 1980. This hypothesis states that the sensory input which is best suited to a task will be favored. Hence, since audition has a greater temporal acuity than vision, auditory input dominated over visual input in temporal judgments (Welch et al., 1986). Ernst and Bülthoff (2004) refined the hypothesis, pointing out that sensory dominance is not dependent on the appropriateness of the modality or the stimulus, but is determined rather by the reliability by which a certain task-relevant property can be estimated within a specific modality from a given stimulus. Based on this account, they hypothesized that visuo-haptic integration occurs in a statistically optimal fashion, minimizing the variance in the final perceptual estimate. Statistically optimal visuo-haptic integration would hence result in a task-dependent weighting of visual and haptic information, determined by the reliability of the information obtained by vision or haptics for the task at hand. Therefore, since visual (macro spatial) size information is more reliable, size perception showed visual capture. However, changing the reliability of visual size information by adding noise increased 
the weight of the haptic information in determining the eventual percept (Ernst and Banks, 2002). Accordingly, tactile judgments of fine surface texture have been shown to be superior to visual judgments (Jones and O’Neill, 1985; Heller, 1989). While visual assessment of roughness was modulated by incongruous tactile stimulation, tactile roughness judgments were insensitive to visual interference, even when the visual distractors were more discriminable than the tactile targets (Guest and Spence, 2003).

The reliability or appropriateness of the estimates of different object properties is also reflected by the modality that participants choose to use for tasks involving these properties. Participants judging visuo-haptic object properties used vision alone for macro spatial geometric properties and course material judgments; in contrast, to perceive subtle differences in material properties, they used touch (Klatzky et al., 1993). Moreover, when participants haptically sorted objects in the presence of vision, they tended to categorize based on structural objects features, whereas purely haptic sorting changed the sorting strategy towards the use of material features (Klatzky et al., 1987).

\section{Spatial reference frames}

Imagine trying to put up a picture in a room. Usually it is quite hard to get it parallel to the edges of the wall. When you step back, something that looked horizontal suddenly turns out to be not straight at all! Now, imagine performing the same task in the dark...

A large body of research has demonstrated systematic errors in haptic parallelity tasks, in which blindfolded participants are asked to make a test bar parallel to a manually explored reference bar at another location (Kappers, 1999; Newport et al., 2002; Kappers 2003; Kaas and Van Mier, 2006; chapter 2). Whereas vision is characterized by a large field of view and the ability to quickly detect and relate light from different distant sources, the span of our hands is limited and hand movement velocity is more constrained. Hence, the relatively poor spatial performance of the tactile sense, as in haptic parallel matching, might be related to the fact that it is very difficult to integrate the inputs from multiple, spatially separate local contacts. Making two distant tactile orientations parallel to each other requires hand position in space to be factored out, with the prerequisite that exact information of the hand in space is available. However, kinesthetic estimation of hand position might not be accurate enough for this purpose (Vindras et al., 1998; Haggard et al., 2000; Zuidhoek et al., 2005). Accordingly, error direction in haptic parallel matching is correlated with the discrepancy between the natural orientation of the hands at reference and test bar locations (Kappers, 2004; Zuidhoek et al, 2005; figure 3). And just as the orientation difference between the hands increases when the hands are moved away from the body's midsagittal plane, error size also increases with inter manual distance (e.g. Kappers et al., 2004). To achieve veridical parallel matching, the existing spatial relations between the objects in external space need to be preserved, and the spatial representation is to be independent of hand position and orientation. This can only be accomplished when the orientation is coded in an allocentric reference frame, fixed in external space. However, sensory input is initially coded in egocentric frames, fixed to the perceiver and the sensory receptors by which it is acquired. It has been proposed that the tactile sense uses a reference frame intermediate between an egocentric, hand-centered reference frame 
and an allocentric, world-based frame (Flanders and Soechting, 1995; Kappers, 2004; figure 3).

A reference frame is a set of axes crossing at a certain origin or center, from which it often derives its name. Usually, the axes are orthogonal. The term egocentric is sometimes used interchangeably with the term intrinsic, just like the terms allocentric and extrinsic. Nevertheless, they do not entirely overlap. The trajectory of the hand in space may be coded in an intrinsic reference frame, in terms of muscle commands, or in an extrinsic frame, as a trajectory through external (visual) space. However, in both cases, the reference frame in which the trajectory is coded is hand-dependent.

The characteristics (e.g. position) of a certain object can be represented as a combination of numbers (coordinates), referring to the object's position on the axes of a reference frame, which can be identified in the external world, but also at the level of sensory receptors, single neurons, neuronal populations or joints and muscles. At a neural level, each cell has a particular response field, i.e. a set of (abstract) features to which it responds by increasing its firing rate. In the case of retinal cells, each cell responds to a certain region of space, and a certain wave length. When the eye moves, the response field also moves in external space, but stays fixed with respect to the retina. This is also true for cells in the primary visual cortex (V1). Therefore, it is justified to say that V1 uses a retinotopic reference frame. To achieve a stable spatial percept, information of the retinal position of an object needs to be combined with information on gaze orientation, the position of the head on the body and the body in space. These transformations might involve specific regions of the parietal cortex (Culham and Kanwisher, 2001; Andersen and Buneo, 2002)

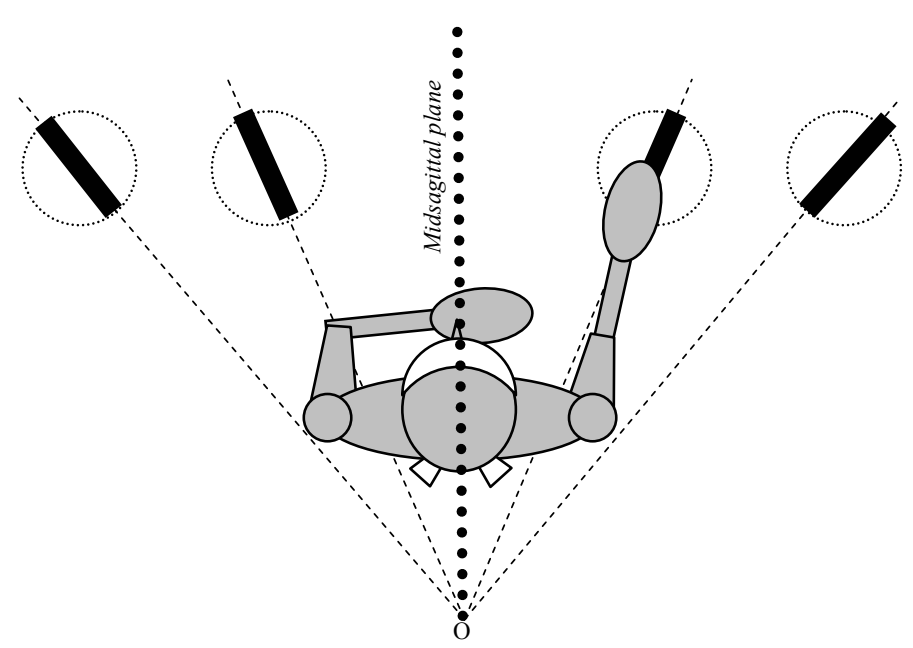

Figure 3. Schematic view of the typical pattern of deviations in haptic parallel matching. The "O" at the bottom represents the hypothetical center of the "intermediate" reference frame governing haptic spatial matching.

In contrast to the large systematic errors in haptic parallel matching, high accuracy is reached in haptic mirror matching, which can be realized in an egocentric, hand- 
centered frame as well as an allocentric reference frame (Kappers, 2004; Newport et al., 2002). This finding supports that it is indeed an inability to account for hand orientation which causes the large errors in haptic parallel matching. In the haptic mirror task, participants turn a test bar to an orientation which is the mirror image of the reference orientation with respect to the midsagittal body plane. Hence, the biasing effect of hand orientation need not be factored out, since it is opposite for both hands. Choosing an optimal reference frame simplifies the task at hand. An egocentric frame might suffice when performing a reach towards a body part, whereas allocentric coding is required for a position-independent description of objects with respect to other objects or other perceivers. Visually guided reaching might be planned in an eyecentered reference frame, because hand trajectory is easily computed by subtracting the eye-centered location of the target and the hand (Buneo et al., 2002). Evidence from single cell recordings and behavioral experiments in humans indicate that reaches are coded at multiple levels, in multiple reference frames, and that different representations come to dominate behavior depending on the circumstances (Battaglia-Mayer et al., 2003). In the dark, pointing showed hand-correlated errors, while the errors when pointing with visual feedback were correlated with gaze direction. Task instruction also influenced spatial error distributions: when participants were told that they were pointing at different locations of an imaginary line, the errors were correlated with this hypothetical line.

In the same vein, parallel and mirror matching might use the same intermediate reference frame in a purely haptic condition, but changing the circumstances by adding non-informative vision (Zuidhoek et al., 2004; Newport et al., 2002) indicated that intrinsic, egocentric frames might be preferred in haptic mirror matching and head- or eye-centered frames in parallel matching.

\section{Haptic working memory}

The integration of sequentially obtained tactile percepts into a unitary object representation draws upon working memory, a process which maintains information online for subsequent use. The concept of working memory was introduced by Baddeley in the 1970s, replacing the idea of a separate short-term store with a limited span. Instead, capacity limits were proposed to depend on the speed of information rehearsal.

To date, most research on the neural substrate of (spatial) working memory has employed stimulation in the visual or auditory modality (e.g., Owen, 1997; Haxby et al., 2000; Wager and Smith, 2003), and only few authors have ventured into the domain of haptic working memory (Stoeckel et al., 2003, 2004; Ricciardi et al., 2005). Nevertheless, there is some evidence that haptic working memory is characterized by three different processing stages, similar to other modalities. Most sensory details of a tactile stimulus are retained up to $500 \mathrm{~ms}$ after stimulus offset. During this stage, perceptual masking can occur, raising the intensity required for detection of a subsequent stimulus (Loomis, 1981; Craig and Rollman, 1999). The second stage is characterized by vivid recollections of uncategorized stimulus information. Although some information is lost, retention is not affected by interfering tasks until approximately 5 seconds after stimulus offset. The third stage lasts up to 30 seconds after stimulus offset, and requires rehearsal mechanisms to preserve only a limited set of feature values (Burton and Sinclair, 2000). Behaviorally, tactile retention up to at least $15 \mathrm{sec}$ has been shown 
(Bowers et al., 1990; Kiphart et al., 1992; Burton and Sinclair 2000; Woods et al., 2004). Zuidhoek et al. (2003) reported that a delay of 10 seconds reduced parallel matching error, being most pronounced in the condition with $120 \mathrm{~cm}$ inter manual distance.

Which brain areas are involved in tactile working memory? Anatomic studies in monkeys showed that tactile information from the somatosensory cortex is directed ventrally through the insula to the frontal cortex for short-term storage (Burton and Sinclair, 2000; Constantinidis and Procyk, 2004). Other non-human primate studies of working memory in the somatosensory domain have shown sustained responses in primary somatosensory regions (Zhou and Fuster, 1996), parietal area 5 (Koch and Fuster, 1989) and prefrontal cortical areas (Romo et al., 1999). Human fMRI studies found activation in ventral prefrontal cortex for maintenance of the oblongness of a manually explored object (Stoeckel et al., 2003), and left anterior intraparietal sulcus for maintenance of kinesthetic information for subsequent haptic shape discrimination (Stoeckel et al., 2004). Dorsal prefrontal activation was observed when a spatiotemporal pattern of pressure pulses had to be maintained online (Numminen et al., 2004).

\section{Outline and research aims of the current thesis}

The current thesis aims to investigate the spatial reference frames in haptic spatial matching (chapters 2-4), as well as the neuronal bases of haptic working memory for object orientation (chapter 5) and fine surface texture or roughness (chapter 6). The dominant reference frames in haptic parallel and mirror matching were probed in a series of psychophysical experiments, whereas fMRI was used to reveal the neural bases of haptic working memory.

It has been suggested that a common intermediate reference frame is involved in parallel and mirror matching (Kappers, 2004). This frame would result from a participantdependent weighted average of an egocentric, hand-centered frame, and an allocentric, world-based frame (Kappers, 2003). However, on the other hand, it was found that parallel matching benefits from non-informative visual information (Newport et al. 2002; Zuidhoek et al. 2004) and orienting towards the exploration hand (Zuidhoek et al. 2004). In addition, studies using pointing tasks showed that optimal reference frames come to dominate behavior (Battaglia-Mayer et al., 2003), suggesting a flexible weighting of reference frames, adapted for the task and the information at hand. The behavioral studies presented in chapters 2, 3 and 4 varied several parameters, such as inter manual distance, visual information and body posture, to investigate their effect on matching errors for the parallel and mirror task in right-handed participants. We assumed that a when a change in matching accuracy would be induced by these parameters, this would indicate that the reference frame underlying performance was dependent on that parameter.

Chapter 2 investigates the effect of distance between reference and test bar $(30$ or 60 $\mathrm{cm})$ and delay $(0.5,5$ and $10 \mathrm{~s})$ between exploration and matching and gender on haptic matching accuracy. It was expected that increasing the distance would have a detrimental effect on parallel matching, but not on mirror matching, whereas performance might be relatively robust across the different delays (Burton and Sinclair, 2000), or might even improve (Zuidhoek et al., 2003). Finally, males were expected to outper- 
form females, in line with gender differences previously reported in haptic spatial matching (e.g. Kappers et al., 2003).

Chapter 3 studies the effect of visual information during the exploration phase of delayed (5 s) haptic parallel and mirror tasks on matching accuracy and time, and investigated the accuracy of haptic ipsilateral matching, in which the location and the hand used in the matching phase were the same as during the exploration phase. We expected a beneficial effect of visual information on haptic parallel matching, relying more on allocentric frames, and no effect or a detrimental effect on mirror matching, relying more on egocentric frames. In addition, we expected that haptic ipsilateral parallel matching and haptic contralateral mirror matching would be achieved with the same accuracy, supporting the idea that both tasks are performed in a similar egocentric frame.

In Chapter 4, body posture is manipulated, resulting in a distortion of body-centered reference frames while head position remained the same. Right-handed participants performed haptic parallel and mirror matching either with their body forward, in a natural position, or turned sideward, at a 90 degree angle with the midsagittal plane of the head, which was maintained in a frontal position. In addition, in half of the trials the left hand was used for exploration of the reference bar while the right hand simultaneously turned the test bar, and vice versa in the other half of the trials. The expectation was that mirror matching performance, relying more heavily on egocentric frames, would show higher errors in the legs sideward condition, whereas parallel matching would remain stable. It was expected that performance might be better when the left hand would be used for exploration, in the light of findings of superior tactuo-spatial abilities of the left hand and right hemisphere (e.g. Dodds et al., 1978).

The fMRI experiment described in Chapter 5 used the same haptic spatial matching task as described in chapter 2 , with a distance of $30 \mathrm{~cm}$ between the bars. The aim was to reveal the neural substrate of haptic working memory for orientation, focusing on those areas for which the activation showed positive correlation with delay length. Based on the description of tactile memory given by Burton and Sinclair (2000) in their review article, different areas were expected to be dominant at different time points in the delay. We expected activity to evolve from prominent involvement of primary sensory and motor cortex just after stimulus offset, to activation of somatosensory association areas in the parietal cortex and regions of prefrontal cortex in later stages. In addition, the neural substrate involved in parallel and mirror matching was compared, expecting to see higher activation in somatosensory areas in the mirror task and higher activation in posterior parietal or parieto-occipital areas for parallel matching, potentially converging for longer delay lengths.

Finally, chapter 6 aims to elucidate the brain areas involved in working memory for tactile surface texture (i.e. roughness). Right-handed participants felt the roughness of one, two, three, or four strips of sandpaper. After a delay of 10 or 12 seconds, they were again presented with a strip of sandpaper, and had to decide whether this stimulus had the same surface texture as one of the stimuli in the previous set. In two control conditions, participants only performed the finger movements, or just remembered which stimulus locations were filled. Areas subserving texture working memory were assumed to show higher delay-activity for higher stimulus loads, as well as higher 
activity for remembering texture versus location, and for texture memory trials versus movement control trials. We expected to find activation in secondary somatosensory areas or anterior parts of the parietal cortex when participants were remembering tactile texture, and no involvement of parieto-occipital areas for this micro spatial object characteristic.

\section{References}

Amedi A, Malach R, Hendler T, Peled S, Zohary E (2001) Visuo-haptic object-related activation in the ventral visual pathway. Nat Neurosci 4: 324-330.

Amedi A, Jacobson G, Hendler T, Malach R, Zohary E (2002) Convergence of visual and tactile shape processing in the human lateral occipital complex. Cereb Cortex 12: 1202-1212.

Andersen RA, Buneo CA (2002). Intentional maps in posterior parietal cortex. Ann Rev Neurosci 25: 189220.

Battaglia-Mayer A, Caminiti R, Lacquaniti F, Zago M (2003) Multiple levels of representation of reaching in the parieto-frontal network. Cereb Cortex 13: 1009-1022.

Bodegård A, Geyer S, Grefkes C, Zilles K, Roland P (2001) Hierarchical Processing of Tactile Shape in the Human Brain. Neuron 31: 317-328.

Bohlhalter S, Fretz C, Weder B (2002) Hierarchical versus parallel processing in tactile object recognition: a behavioural-neuroanatomical study of aperceptive tactile agnosia. Brain 125: 2537-2548.

Bowers RL, Mollenhauer MS, Luxford J (1990) Short-term memory for tactile and temporal stimuli in a shared-attention recall task. Percept Mot Skills 1990 70: 903-13.

Buneo CA, Jarvis MR., Batista AP, Andersen RA (2002). Direct visuomotor transformations for reaching. Nature 416: 632-636.

Burton H, Sinclair RJ (2000) Attending to and remembering tactile stimuli: A review of brain imaging data and single-neuron responses. J Clin Neurophysiol 17: 575-591.

Constantinidis C, Procyk E (2004) The primate working memory networks. Cogn Affect Behav Neurosci 4: 444-465.

Craig JC, Rollman GB (1999) Somesthesis. Annu Rev Psychol 50:305-331.

Culham JC, Kanwisher NG (2001) Neuroimaging of cognitive functions in human parietal cortex. Current Opinion in Neurobiology 11: 157-163.

Dodds AG (1978) Hemispheric differences in tactuo-spatial processing. Neuropsychologia 16:247-250.

Donald, M. (1997). Precis of Origins of the modern mind: Three stages in the evolution of culture and cognition. Behavioral and Brain Sciences 16 (4): 737-791.

Ernst MO, Banks MS (2002) Humans integrate visual and haptic information in a statistically optimal fashion. Nature 415: 429-433.

Ernst MO, Bülthoff HH (2004) Merging the senses into a robust percept. Trends Cogn Sci 8: 162-169.

Flanders M, Soechting JF (1995) Frames of reference for hand orientation. J Cog Neurosci 7: 182-195.

Gardner EP, Kandel ER (2000) Touch. In: Kandel ER, Schwartz JH, Jessell TM (eds) Principles of Neural Science. McGraw-Hill, New York: 451-471.

Guest S, Spence C (2003) Tactile dominance in speeded discrimination of textures. Exp Brain Res 150: 201-207.

Haggard P, Newman C, Blundell J, Andrew H (2000) The perceived position of the hand in space. Percept Psychophys 62:363-377. 
Haxby JV, Petit L, Ungerleider LG, Courtney SM (2000) Distinguishing the functional roles of multiple regions in distributed neural systems for visual working memory. Neuroimage 11: 380-391.

Heller MA (1989) Picture and pattern perception in the sighted and the blind: the advantage of the late blind. Perception 18: 379-389.

James TW, Humphrey GK, Gati JS, Servos P, Menon RS, Goodale MA (2002) Haptic study of threedimensional objects activates extrastriate visual areas. Neuropsychologia 40, 1706-1714.

Jones B, O'Neil S (1985) Combining vision and touch in texture perception. Percept Psychophys 37: 66-72. Kaas AL, Van Mier HI (2006). Haptic spatial matching in near peripersonal space. Exp Brain Res 170: 403413.

Kaas JH, Nelson, RJ, Sur M, Lin CS, Merzenich MM (1979) Multiple representations of the body within the primary somatosensory cortex of primates. Science 204: 521-523.

Kappers AML (1999) Large systematic deviations in the haptic perception of parallelity. Perception 28:1001-1012.

Kappers AML (2003) Large systematic deviations in a bimanual parallelity task: further analysis of contributing factors. Acta Psychol 114:131-145.

Kappers AML (2004) The contributions of egocentric and allocentric reference frames in haptic spatial tasks. Acta Psychol 117:333-340.

Kiphart MJ, Hughes JL, Simmons JP, Cross HA (1992) Short-trem haptic memory for complex objects. Bull Psychon Soc 30: 212-214.

Klatzky RL, Lederman SJ, Metzger V (1985) Identifying objects by touch: an "expert system". Percept Psychophys 37: 299-302.

Klatzky RL, Lederman S, Reed C (1987) There's more to touch than meets the eye: the salience of object attributes for haptics with and without vision. J Exp Psychol Gen 116: 356-369.

Klatzky RL, Lederman SJ, Matula DE (1993) Haptic exploration in the presence of vision. J Exp Psychol Hum Percept Perform 19: 726-743.

Klatzky RL, Lederman SJ (1995) Identifying objects from a haptic glance. Percept Psychophys 57: 11111123.

Koch C, Fuster JM (1989) Unit activity in monkey parietal cortex related to haptic perception and temporary memory. Exp Brain Res 76: 292-306.

Lederman SJ, Klatzky RL (1987) Hand movements: a window into haptic object recognition. Cognitive Psychology 19: 342-368.

Lederman SJ, Klatzky RL (1997) Relative availability of surface and object properties during early haptic processing. J Exp Psychol Hum Percept Perform 23: 1680-1707.

Lederman SJ, Klatzky RL (2004) Multisensory Texture Perception. In: Calvert G, Spence C, Stein BE (eds) The handbook of Multisensory Processes. MIT press, Cambridge MA: pp 107-122.

Loomis JM (1981) Tactile pattern perception. Perception 10:5-27.

Merabet L, Thut G, Murray B, Andrews J, Hsiao S, Pascual-Leone A (2004) Feeling by sight or seeing by touch? Neuron 42: 173-179.

Newport R, Rabb B, and Jackson SR (2002). Noninformative vision improves haptic spatial perception. Curr Biol 12:1661-1664.

Numminen J, Schurmann M, Hiltunen J, Joensuu R, Jousmaki V, Koskinen SK, Salmelin R, Hari R (2004) Cortical activation during a spatiotemporal tactile comparison task. Neuroimage 22: 815-821.

Owen AM (1997) The functional organization of working memory processes within human lateral frontal cortex: The contribution of functional neuroimaging. European Journal of Neuroscience 9: 1329-1339. 


\section{6 | Chapter 1}

Pietrini P, Furey ML, Ricciardi E, Gobbini MI, Wu WH, Cohen L, Guazzelli M, Haxby JV (2004) Beyond sensory images: Object-based representation in the human ventral pathway. Proc Natl Acad Sci U S A 101:5658-5663.

Randolph M, Semmes J (1974) Behavioral consequences of selective subtotal ablations in the postcentral gyrus of Macaca mulatto. Brain Res 70: 55-70.

Ricciardi E, Bonino D, Gentili C, Sani L, Pietrini P, Vecchi T (2006) Neural correlates of spatial working memory in humans: a functional magnetic resonance imaging study comparing visual and tactile processes. Neuroscience 139: 339-349.

Roland PE, O'Sullivan B, Kawashima R (1998) Shape and roughness activate different somatosensory areas in the human brain. Proc Natl Acad Sci U S A. 95: 3295-3300.

Romo R, Brody CD, Hernandez A, Lemus L (1999) Neuronal correlates of parametric working memory in the prefrontal cortex. Nature 399: 470-473.

Sadato N, Pascual-Leone A, Grafman J, Ibanez V, Deiber MP, Dold G, Hallett M (1996) Activation of the primary visual cortex by Braille reading in blind subjects. Nature 380: 526-528.

Sathian K, Burton H (1991) The role of spatially selective attention in the tactile perception of texture. Percept Psychophys 50: 237-248.

Sathian K, Zangaladze A, Hoffman JM, Grafton ST (1997): Feeling with the mind's eye. Neuroreport 8:3877-3881.

Stoeckel MC, Weder B, Binkofski F, Buccino G, Shah NJ, Seitz RJ (2003) A fronto-parietal circuit for tactile object discrimination: An event-related fMRI study. Neuroimage 19: 1103-1114.

Stoeckel MC, Weder B, Binkofski F, Choi H-J, Amunts K, Pierperhoff P, Shah NJ, Seitz RJ (2004) Left and right superior parietal lobule in tactile object discrimination. Eur J Neurosc 19: 1067-1072.

Stoesz MR, Zhang M, Weisser VD, Prather SC, Mao H, Sathian K (2003) Neural networks active during tactile form perception: common and differential activity during macrospatial and microspatial tasks. Int J Psychophysiol 50: 41-49.

Vindras P, Desmurget M, Prablanc C, Viviani V (1998) Pointing errors reflect biases in the perception of the initial hand position. J Neurophysiol 79: 3290-3294.

Wager TD, Smith EE (2003) Neuroimaging studies of working memory: A meta-analysis. Cogn Affect Behav Neurosci 3: 255-274.

Welch RB, DuttonHurt LD, Warren DH (1986) Contributions of audition and vision to temporal rate perception. Percept Psychophys 39: 294-300.

Woods AT, O'Modhrain S, Newell FN (2004) The effect of temporal delay and spatial differences on cross-modal object recognition. Cogn Affect Behav Neurosci 4: 260- 269.

Zhang M, Mariola E, Stilla R, Stoesz M, Mao H, Hu X, Sathian K (2005) Tactile Discrimination of Grating Orientation: fMRI Activation Patterns. Hum Brain Mapp 25: 370 -377.

Zangaladze A, Epstein CM, Grafton ST, Sathian K (1999) Involvement of visual cortex in tactile discrimination of orientation. Nature 401:587-590.

Zimbardo P, McDermott M, Jansz J, Metaal N (1995) Psychology: a European text. Haper Collins, London, UK.

Zhou YD, Fuster JM (1996) Mnemonic neuronal activity in somatosensory cortex. Proc Natl Acad Sci U S A 93: 10533-10537.

Zuidhoek S, Kappers AM, van der Lubbe RH, Postma A (2003) Delay improves performance on a haptic spatial matching task. Exp Brain Res 149: 320-330.

Zuidhoek S, Visser A, Bredero ME, Postma A (2004) Multisensory integration mechanisms in haptic space perception. Exp Brain Res 157: 265-268. 
General introduction | 27

Zuidhoek S, Kappers AML, Postma A (2005) Effects of hand orientation and delay on the verbal judgment of haptically perceived orientation. Perception 34: 741-755. 



\section{CHAPTER 2}

\section{Haptic spatial matching}

Based on: Kaas AL, Van Mier HI (2006). Haptic spatial matching in near peripersonal space. Exp Brain Res 170: 403-413. 


\begin{abstract}
Research has shown that haptic spatial matching at intermanual distances over $60 \mathrm{~cm}$ is prone to large systematic errors. The error pattern has been explained by the use of reference frames intermediate between egocentric and allocentric coding. This study investigated haptic performance in near peripersonal space, i.e. at intermanual distances of 60 $\mathrm{cm}$ and less. Twelve blindfolded participants (6 males and 6 females) were presented with two turn bars at equal distances from the midsagittal plane, 30 or $60 \mathrm{~cm}$ apart. Different orientations (vertical/horizontal or oblique) of the left bar had to be matched by adjusting the right bar to either a mirror symmetric $(/ \backslash)$ or parallel $(/ /)$ position. The mirror symmetry task can in principle be performed accurately in both an egocentric and an allocentric reference frame, whereas the parallel task requires an allocentric representation. Results showed that parallel matching induced large systematic errors which increased with distance. Overall error was significantly smaller in the mirror task. The task difference also held for the vertical orientation at $60 \mathrm{~cm}$ distance, even though this orientation required the same response in both tasks, showing a marked effect of task instruction. In addition, men outperformed women on the parallel task. Finally, contrary to our expectations, systematic errors were found in the mirror task, predominantly at $30 \mathrm{~cm}$ distance. Based on these findings, we suggest that haptic performance in near peripersonal space might be dominated by different mechanisms than those which come into play at distances over 60 centimetres. Moreover, our results indicate that both inter-individual differences and task demands affect task performance in haptic spatial matching. Therefore, we conclude that the study of haptic spatial matching in near peripersonal space might reveal important additional constraints for the specification of adequate models of haptic spatial performance.
\end{abstract}




\section{Haptic spatial matching}

$\mathbf{F}_{\text {or successful interaction with objects in peripersonal space, an accurate representa- }}$ tion of this space seems indispensable. However, evidence indicates that both visual (Cuijpers et al. 2000; Hermens and Gielen 2003; Bingham et al., 2004; Henriques et al., 2005) and haptic (Kappers, 1999, 2002, 2004; Kappers and Koenderink, 1999, Luyat et al., 2001; Newport et al., 2002; Henriques et al., 2004) spatial representations are prone to systematic errors. When participants are instructed to make line segments parallel to each other using active touch (haptic parallel matching), the error pattern indicates that what is perceived as parallel systematically deviates from what is physically parallel (Kappers 1999, 2002, 2004; Kappers and Koenderink, 1999; Newport et al., 2002; Zuidhoek, et al. 2003). Counter clockwise errors are performed when the orientation of bars to the left of the midsagittal plane are to be made parallel to a bar at a location to the right. Conversely, clockwise errors occur when making bars to the right parallel to bars to the left of the midsagittal plane (Kappers, 2003, 2004). The magnitude of the errors increases at greater distances between the bars (Kappers, 1999, 2002; Kappers and Koenderink, 1999; Zuidhoek et al., 2003, 2004).

It might be argued that errors in haptic spatial matching result from a lack of sensitivity or acuity in the haptic domain. However, research by Henriques and Soechting (2003) suggests that haptic perception is comparable to visual perception in terms of acuity, stressing the importance and sensitivity of the haptic system. In spite of haptic acuity, systematic errors are observed in haptic spatial matching tasks. This indicates that the errors arise from non-veridical haptic spatial representations which guide task execution. Hermens et al. (2006) had participants report the orientation of haptically perceived bars as a clock time, and found that the errors were much smaller than the errors on a haptic parallel matching task. In addition, she found that the errors made when turning bars to a predefined orientation were also much smaller than haptic parallel matching errors. Hermens et al. concluded that haptic matching errors do not originate in the haptic perception or production of orientations, but rather in the transfer of the perceived bar orientation to the location of the test bar.

The analysis of systematic error patterns has been used in attempts to reveal the reference frames in which these internal representations are coded (McIntyre et al. 2000, Carrozzo et al. 2002). The directionality of the error pattern in haptic spatial matching as described above has been hypothesized to reflect the influence of an egocentric 
reference frame. Egocentric reference frames are centred on, or referred to the participant's body, as opposed to allocentric reference frames, which are linked to extrabodily cues, like the direction of the gravitational pull or (haptic) contextual cues (Luyat et al., 2001; Batista, 2002; see figure 1).

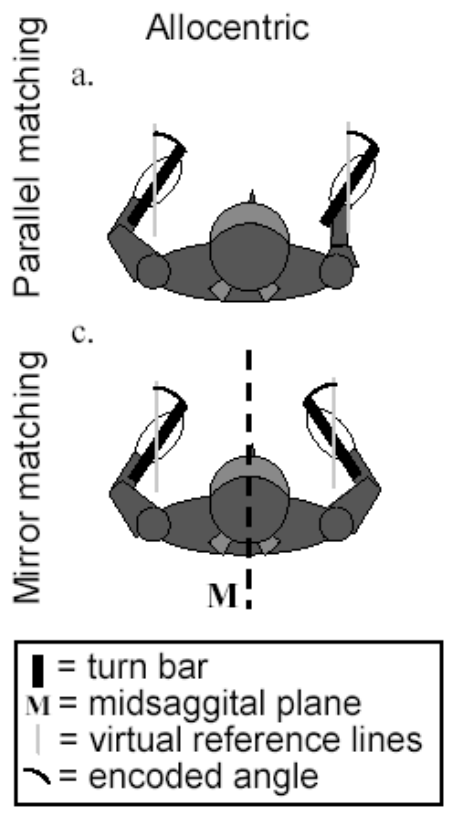

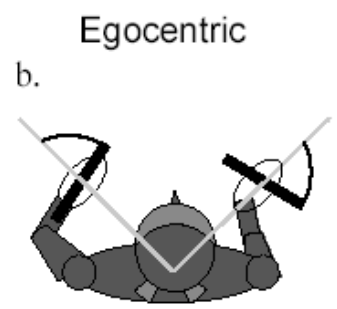

d.

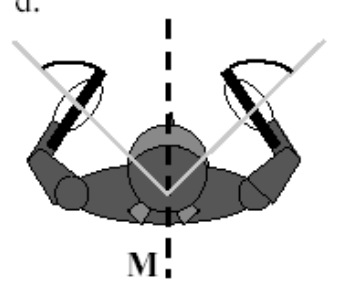

e.

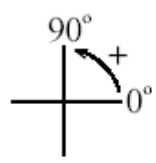

Figure 1. Schematic overview of the parallel and mirror task and hypothetical responses in a purely allocentric or purely egocentric reference frame. The participant's hands have been made transparent to allow view of the turn bars. Grey lines indicate imaginary reference lines through the centre of the turn bar, originating from the participants ego centre or parallel to allocentric reference frame axes. The reference line corresponds to the axis through the pivot of the turnbar that runs parallel to the participant's midsagittal plane in the allocentric reference frame, whereas it corresponds to the line through the participant's ego centre and the pivot of the turnbar in the egocentric reference frame. As opposed to the parallel task, performance of the mirror task requires different processing of the encoded angle. In the mirror task, the angle has to be subtracted from the orientation of the reference line through the pivot of the turnbar, whereas it needs to be added in the parallel task.

a: Parallel matching would be veridical in a purely allocentric frame. b: However, in an egocentric reference frame, parallel matching would show strong deviations, correlated to the natural position of the hand at the location of the test bar, $\mathrm{c}$ and $\mathrm{d}$ : whereas mirror matching would be veridical in both an allocentric and an egocentric frame. e: Definition of the $0^{\circ}$ orientation and the sign convention used in this study.

However, although the use of an egocentric reference frame explains the directional bias of the haptic parallel matching errors very well, it does not explain the size of the errors. That is, the size of the errors which are empirically observed is smaller than to be expected when a purely egocentric frame would be used. Therefore, haptic spatial matching performance has been proposed to be the product of a reference frame intermediate between an allocentric frame, fixed in external space, and an egocentric frame, centred on the midsagittal plane (Flanders and Soechting, 1995; Kappers, 2003, 2004). Although this explanation proved to be very powerful in describing global error 
patterns and individual differences, the fact that the magnitude of the errors in haptic parallel matching tasks is not the same for different orientations still remains unexplained. Vertical and horizontal (cardinal) orientations are usually more accurately processed than oblique orientations. This so called 'oblique effect', first described by Appelle (1972), is observed in the precision (variability) of the responses and the average error size in both visual and haptic tasks, in adults as well as in infants, children, and adolescents (Flanders and Soechting, 1995; Gentaz and Hatwell, 1995; 1998; Kappers, 1999, 2002, 2003, 2004; Gentaz et al., 2001; Luyat et al., 2001; Luyat and Gentaz 2002; Cuijpers et al., 2003; Van Mier et al., 2003; Gentaz and Streri, 2004; Fraussen et al., 2004; Fernandez and Van Mier, 2005).

The extent to which haptic performance is dominated by egocentric representations is participant-dependent, as indicated by great inter-individual variation in error size on the parallel matching task. The variation could not be accounted for by factors such as arm length, shoulder width, familiarity with or specific training on the task (Kappers, 2003; Kappers, 2004: personal communication). However, error size was significantly correlated with gender and profession. That is, male technicians were better at haptic parallel matching than females with administrative jobs, but, interestingly, male physicists also had smaller deviations than female physicists (Kappers, 2003). These findings suggest that the weighting factor for the contribution of the egocentric reference frame might be variable across participants and different between males and females, but stable across different tasks and conditions.

If the proposed intermediate frame is indeed used in haptic spatial matching tasks, we would expect an average error around zero when the participant's task is to produce the mirror image of a haptically explored line orientation in the midsagittal plane, because this task can be performed accurately in both reference frames that contribute to the intermediate frame (see figure 1, b and d). Additionally, one would expect that distance between the hands would not affect the error size for mirror symmetry matching. With respect to the former, in a study in which participants performed both a mirror and a parallel matching task, Kappers (2004) actually found an average error of zero for mirror symmetry matching at $120 \mathrm{~cm}$. However, in a mirror symmetry experiment at $20 \mathrm{~cm}$ distance, Newport et al. (2002) found clockwise and counter clockwise errors, depending on the orientation of the bar. The errors reported for parallel matching did follow the expectations based on the intermediate reference frame model. Note that Newport's analyses used signed errors, in which any residual perceptual or executive error would cancel to zero, due to its normal distribution around the veridical response. Therefore, no directed errors could have arisen, had the participants' behaviour been guided by the intermediate frame proposed by Kappers, and these unexpected results indicate that different mechanisms might become dominant in near peripersonal space, at distances close to the midsagittal plane. However, since parallel and mirror matching were not compared within the same participants in Newport's study, the results might also be attributed to inter-individual differences.

The present study directly compared parallel and mirror matching tasks in near peripersonal space within the same participant sample. The orientation of a bar on the lefthand side was manually explored, and subsequently matched by turning another bar at the right-hand side, equidistant from the body midline. Blindfolded participants were instructed to either match the bars by making them parallel, i.e. to match the orienta- 
tion in the allocentric, Cartesian coordinates of the outside world, or to create the mirror image of the orientation with respect to the midsagittal plane of the body, i.e. to match the orientation in egocentric, limb-based coordinates. Distance between the bars was either 30 or $60 \mathrm{~cm}$. Based on the predictions following from the intermediate reference frame model, we expected an effect of task (hypothesis 1), i.e. clockwise errors in the parallel task and no systematic errors in the mirror task, because the mirror task, unlike the parallel task, can be performed correctly in both an egocentric and an allocentric reference frame (see figure $1 \mathrm{~b}$ and $\mathrm{d}$ ). Besides this, we expected a task by distance effect (hypothesis 2), in the sense that the mirror task would not be affected by distance, whereas the parallel task would show a greater error size at the large compared to the short distance, due to the increasing mismatch between the true allocentric reference lines and the reference lines in the intermediate reference frame used when performing the task. In addition, we expected differential errors for the $90^{\circ}$ orientation in parallel and mirror matching (hypothesis 3), reflecting the different task instruction, even though this orientation requires exactly the same response in both tasks, and allows no variation in the positioning of the hands. Moreover, based on results from prior studies, we expected an effect of orientation (hypothesis 4) resulting in an "oblique effect" (hypothesis 5), that is, smaller average errors for the $0^{\circ}$ and $90^{\circ}$ orientation (the cardinal orientations) compared to the other, oblique orientations. Using a parallel task, Kappers (2003, 2004) and Van Mier et al. (2003) previously observed a between-subjects reversal in the direction of the oblique effect in participants with the highest average deviations. Those participants showed greater deviations for the cardinal orientations compared to the oblique orientations. Such a reversal would not be unlikely, considering the fact that the "oblique" orientations are defined with respect to a purely allocentric external reference frame. A participant using a more egocentric, intermediate reference frame might experience the externally defined oblique orientations as cardinal orientations. Therefore, we expected a reversal of the oblique effect for participants using a more egocentric reference frame, i.e. with the highest average errors on the parallel task, showing better performance for oblique instead of cardinal orientations (hypothesis 6). Finally, we expected an effect of gender (hypothesis 7), with male participants outperforming female participants.

The current behavioural study was carried out in relation with an fMRI study (Kaas et al., in press; chapter 5) investigating the neural bases of working memory processes related to haptic orientation matching. For this purpose, a delay was introduced between the exploration and the matching phase. The introduction of a delay has previously been reported to improve parallel matching performance (Zuidhoek et al., 2003). It was found that after a $10 \mathrm{sec}$ delay, performance was significantly better than without a delay. This effect was explained by an increasing dominance of the allocentric reference frame after a delay. Hu and Goodale (2000) reported a similar positive effect in grasping movements after a delay of $5 \mathrm{sec}$. To track possible changes in performance due to a delay, delay length was varied from 5 to $10 \mathrm{sec}$. We hypothesized that a delay could potentially eliminate or decrease the difference between the parallel and mirror task (hypothesis 8). 


\section{Materials and methods}

\section{Participants}

Twelve healthy participants (average age $23 \pm 4$ years; six males, six females) were paid to participate in the present study. All participants were undergraduate students at Maastricht University and were strongly right-handed as assessed by a Dutch translation of the Edinburgh Handedness Inventory (Oldfield, 1971). Informed consent was obtained prior to the experiment. Participants had never seen the set-up, were naïve with respect to the stimulus orientations, and were unaware of the objectives of the present study. Participants wore headphones and were blindfolded to prevent visual input from influencing task performance. The study was approved by the local ethics committee and was performed in accordance with the declaration of Helsinki of 1964.

\section{Apparatus and stimuli}

Two turn bars $(10 \times 1 \mathrm{~cm})$ with a blunt plastic needle at each end were mounted on a table. The orientation of the bars could be read with an accuracy of 0.5 degree. The distance between the pivots of the bars was $30 \mathrm{~cm}$ (small) or $60 \mathrm{~cm}$ (large), depending on the experimental condition. The participant was seated on a chair, of which the legs were placed at a fixed position with respect to the table, to ensure that the body symmetry plane coincided with the plane that orthogonally bisected the line between the pivots of the bars. Five participants performed the small distance condition as part of an fMRI experiment (Kaas et al., in press; chapter 5). They lay supine in the scanner, their heads and arms stabilized with foam padding. A small table was placed over the scanner bed below the waistline, with the two turn bars at $35 \mathrm{~cm}$ distance on top. We found no significant differences between participants performing the parallel and mirror task at the small distance condition in or out of the scanner. For reasons of simplicity, we will refer to this distance also as $30 \mathrm{~cm}$ in the remainder of the paper.

\section{Procedure}

Participants were blindfolded throughout practice and experimental trials. They were instructed to feel the orientation of a reference bar positioned to the left of the body with the left hand. After a variable delay, a test bar had to be turned with the right hand to an orientation that matched the orientation of the reference bar. A different matching criterion was used in the two experimental conditions. In the parallel condition, the test bar had to be turned to an orientation parallel to the reference bar. In the mirror condition, the test bar had to be turned to coincide with the mirror image of the left bar with respect to the midsagittal plane of the body. Figure 1 shows a schematic overview of the set-up for both tasks. Each trial was composed of an exploration phase $(1500 \mathrm{~ms})$, a delay phase (short (500ms), intermediate (5000ms) or long $(10000 \mathrm{~ms})$ delay), and a response phase $(2000 \mathrm{~ms})$. Start and end of the exploration and response phases were indicated by auditory signals presented through headphones (pure tones, $1000 \mathrm{~Hz}$ for the exploration phase and $2000 \mathrm{~Hz}$ for the response phase). Participants were instructed to remove their left hand from the reference bar at the end of the exploration phase and return it to their body. The same procedure was applied regarding the right hand at the end of the response phase. The only explicit instruction given to the participants concerning the starting and return position of the hands was 
that they should touch neither the table nor the bars. In practice, most participants kept their hands in the same resting position at breast height next to their torso throughout the experiment. This allowed them to start exploring or matching as soon as the respective auditory signals sounded.

Before each trial, the experimenter positioned the reference bar in one of six predetermined orientations $\left(0^{\circ}, 30^{\circ}, 60^{\circ}, 90^{\circ}, 120^{\circ}\right.$ and $150^{\circ}$, see figure 2$)$ while the test bar was positioned in either $55^{\circ}$ or $125^{\circ}$, varied across runs. After the response, the experimenter read the orientation indicated by the needle attached to the test bar, and wrote it down. Participants performed a total of 8 runs, two for each combination of task and distance. Each run was made up of a pseudo-random series of 36 trials (3 delays x 6 orientations $\times 2$ repetitions) of either the parallel or mirror task at 30 or 60 $\mathrm{cm}$ distance, resulting in a total of 288 trials. The order of conditions was counterbalanced over participants. Participants had practiced both tasks prior to the experiment, and could perform exploration and response within the time indicated by the auditory signals.

\section{Data analyses}

The orientation parallel to the midsagittal plane was defined as $90^{\circ}$, and the counter clockwise direction as positive. Signed errors were calculated for every trial by subtracting the orientation of the (left) reference bar from the orientation of the (right) test bar. In the mirror condition, matching errors were expressed as deviations from the mirrored reference orientation (i.e. $60^{\circ}=>120^{\circ}$ ). Errors were subsequently normalized to lie between $-89^{\circ}$ and $90^{\circ}$ and averaged over runs.

The errors were analyzed in a 2 (Task: parallel/mirror) x 2 (Distance: $30 / 60 \mathrm{~cm}$ ) x 6 (Orientation: $0^{\circ}, 30^{\circ}, 60^{\circ}, 90^{\circ}, 120^{\circ}$ and $150^{\circ}$ ) x 3 (Delay: 500/5000/10000 ms) withinsubjects repeated measurement ANOVA, with gender as between-subjects factor. In an additional analysis, the six different orientations were clustered in two categories: $0^{\circ}$ and $90^{\circ}$ trials were averaged and entered as cardinal orientations, and all other orientations were aggregated to one oblique variable resulting in a within-factor Obliqueness with 2 levels.

An additional analysis was performed on the variability of the responses, using a delay (3) by task (2) by distance (2) by orientation (6) repeated measures GLM with gender as between factor, and a delay (3) by task (2) by distance (2) by obliqueness (2) repeated measures GLM with gender as between factor.

\section{Results}

Hypothesis 8, concerning the effect of delay was not confirmed. There was no significant main effect of delay on subsequent matching error $(\mathrm{p}<0.148)$, nor were there any significant interactions with delay. We therefore averaged over Delay. Unless otherwise indicated, all $\mathrm{p}$-values reported in the following paragraph originate from the subsequent 2 (Task: parallel/mirror) x 2 (Distance: $30 / 60 \mathrm{~cm}$ ) × 6 (Orientation: $0^{\circ}, 30^{\circ}, 60^{\circ}$, $90^{\circ}, 120^{\circ}$ and $150^{\circ}$ ) ANOVA, with gender as between-subjects factor. Figure 2 shows the mean responses from individual subjects for each task, distance and orientation. 


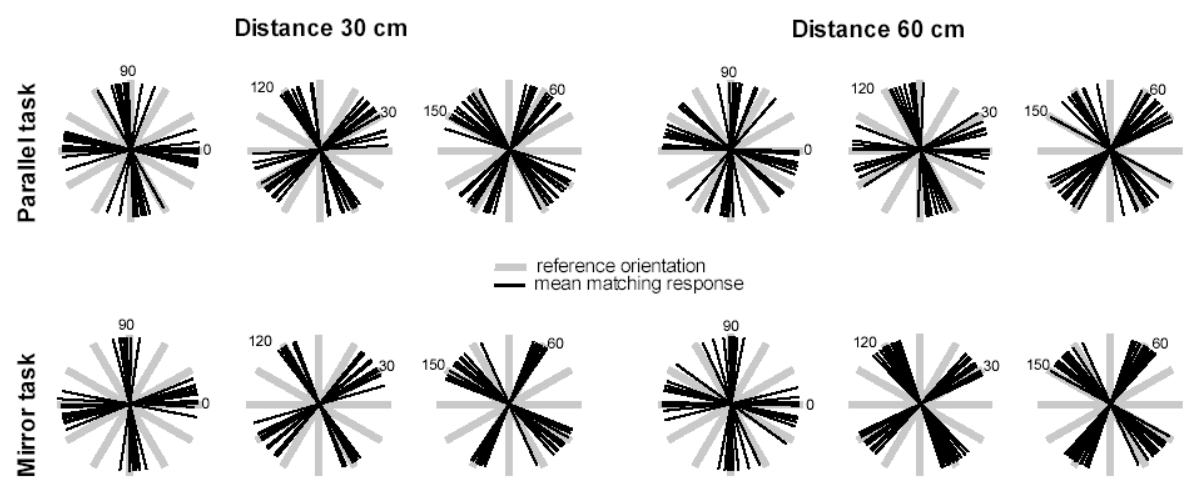

Figure 2. Mean responses of all participants for each orientation, task and distance. To allow the reader to discriminate the responses for each individual reference orientation, each panel shows the responses for two reference orientations separated by at least 60 degrees.

\section{Effects of task, distance and orientation}

A significant main effect was found for Task $(\mathrm{F}(1,10)=16.0, \mathrm{p}<0.003)$, confirming hypothesis 1 . Signed error was smaller for mirror versus parallel matching. The parallel task showed clockwise errors of 11 degrees on average, whereas the mirror task was characterized by an average error of 1 degree clockwise.

Hypothesis 2, concerning the interaction between task and distance was also confirmed. There was a significant interaction $(\mathrm{F}(1,10)=26.8, \mathrm{p}<0.000)$, with the distance effect being most pronounced in the parallel task. Errors during parallel matching increased on average from 2 degrees clockwise to 17 degrees clockwise when the distance between the bars was changed from 30 to $60 \mathrm{~cm}$, while the errors during mirror matching on average increased from 2 degrees counter clockwise at $30 \mathrm{~cm}$ to 3 degrees clockwise at $60 \mathrm{~cm}$. Distance also showed a main effect $(\mathrm{F}(1,10)=75.2, \mathrm{p}<0.000)$. In general, increasing the distance between the bars from 30 to $60 \mathrm{~cm}$ was associated with an error increase of on average 10 degrees in clockwise direction. Counter clockwise errors occurred for mirror matching at a distance of $30 \mathrm{~cm}$, whereas parallel matching at the same distance lead to small clockwise errors. For the $0^{\circ}(\mathrm{t}(11)=2.5), 30^{\circ}(\mathrm{t}(11)$ $=2.3)$ and $90^{\circ}(\mathrm{t}(11)=2.5)$ orientations, these counter clockwise mirror matching errors differed significantly from zero, as demonstrated by one-sided t-tests with an alpha of 5 percent.

Hypothesis 3 , regarding the expected task effect for $90^{\circ}$ trials, was only confirmed for the $60 \mathrm{~cm}$ distance $(\mathrm{F}(1,10)=5.1, \mathrm{p}<0.047)$. This was due to the interaction of Task by Distance which was also significant in the separate analysis including only the $90^{\circ}$ trials $(\mathrm{F}(1,10)=10.7, \mathrm{p}<0.008)$. At $60 \mathrm{~cm}$ distance, parallel matching of the $90^{\circ}$ orientation was associated with an average error of 9 degrees in clockwise direction, which was significantly greater than the 2 degree counter clockwise error observed for mirror matching of the same orientation. By contrast, at a distance of $30 \mathrm{~cm}$ between the bars, both parallel and mirror matching of the $90^{\circ}$ orientation induced a counter clockwise error: 6 degrees for parallel matching and 4 degrees for mirror matching, resulting in a non-significant difference $(F(1,10)=0,231 ; \mathrm{p}<0,641)$. 
The significant main effect for Orientation $(\mathrm{F}(5,50)=12.7, \mathrm{p}<0.000)$ confirmed the fourth hypothesis. When looking at the average across tasks and distances, orientations of $120^{\circ}$ and $150^{\circ}$ resulted in the largest deviations, while $30^{\circ}$ and $90^{\circ}$ were performed most accurately. Orientation interacted with both Task $(\mathrm{F}(5,50)=4.0, \mathrm{p}<0.004)$ and Distance $(\mathrm{F}(5,50)=2.9, \mathrm{p}<0.022)$ (see figure 3 ). The task effect was largest at $30^{\circ}$ and smallest at $90^{\circ}$, with 17 degrees and 5 degrees respectively. The distance effect was largest at $0^{\circ}$ (15 degrees) and smallest at $150^{\circ}$ (4 degrees). In addition, there was a three-way Task x Distance x Orientation interaction $(F(5,50)=4.7, \mathrm{p}<0.001)$.

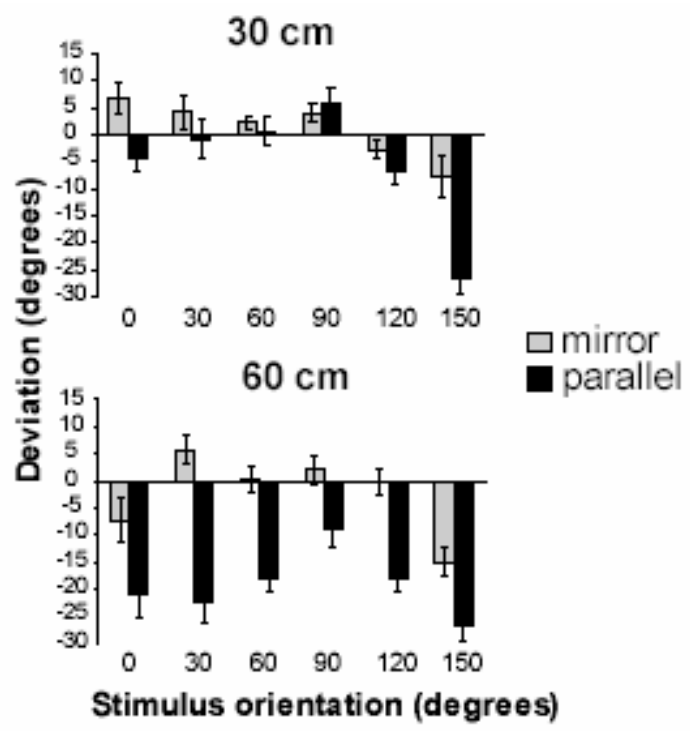

Figure 3. Mean signed error for each task, intermanual distance and reference bar orientation. Error bars represent the standard error of the mean. Mean signed error was calculated by subtracting the orientation of the left reference bar from the orientation of the right test bar.

\section{Effect of obliqueness}

In line with our fifth hypothesis, the analysis regarding the separate orientations assigned to the categories cardinal or oblique, showed that oblique orientations generally yielded more clockwise (negative) deviations than oblique orientations $(F(1,10)=11.6$, $\mathrm{p}<0.007)$ with an average deviation of 3 degrees clockwise for cardinal and 7 degrees clockwise for oblique orientations (see figure 4). However, in the sense of absolute error sizes, we observed a (within-subjects) reversal of the direction of the oblique effect in the mirror task compared to the parallel task. By this, we mean that cardinal orientations in the mirror task showed a greater error than oblique orientations, especially at the $30 \mathrm{~cm}$ distance. At this distance, an error of 5 degrees was observed in counter clockwise direction for cardinal orientations, and of 1 degree clockwise for oblique orientations. At $60 \mathrm{~cm}$, the cardinal error for mirror matching was 3 degrees clockwise, which was only slightly bigger than the 2 degree clockwise oblique error. By contrast, in the parallel task, increasing the distance between the bars by $30 \mathrm{~cm}$ caused 
significantly larger clockwise errors for both cardinal and oblique orientations. This resulted in a Task x Distance x Obliqueness interaction $(\mathrm{F}(1,10)=9.4, \mathrm{p}<0.012)$.

\section{Obliqueness $\mathrm{x}$ task $\mathrm{x}$ distance}

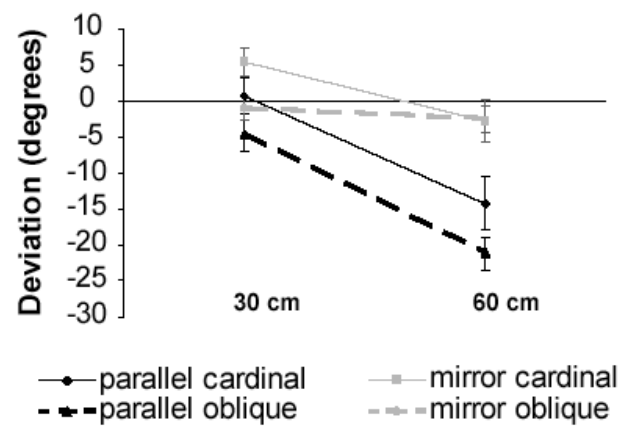

Figure 4. Mean signed errors and standard errors displayed for task, intermanual distance, and cardinal or oblique reference bar orientations.

As can be seen in figure 5, hypothesis 6 was not confirmed. We did not observe a reversal of the oblique effect for participants with the highest overall average error. Almost all participants showed greater deviations for the oblique compared to the cardinal orientations in the parallel task. However, fitted linear trend lines confirmed that the difference between the errors for cardinal and oblique orientations was smaller for participants with larger average errors. When interpolating the fitted linear trend lines for the parallel task at $60 \mathrm{~cm}$, the oblique effect would be expected to reverse for participants with an average error over approximately -38 degrees as can be seen in figure 5 .

\section{Effect of gender}

Hypothesis 7, concerning better performance of male participants, was confirmed. On average, men outperformed women on both haptic matching tasks $(p<0.032)$, with deviations of 2 degrees clockwise for men and 10 degrees clockwise for women. There was a trend towards a Gender x Task interaction $(\mathrm{p}<0.066)$, indicating that the effect of gender was most pronounced for parallel matching, where average errors of 5 degrees clockwise were observed for male participants versus 17 degrees clockwise for female participants (see figure 6).

\section{Precision effects}

In the additional analysis on the variation of the responses, using a delay (3) by task (2) by distance (2) by orientation (6) repeated measures GLM with gender as between factor, we found significant main effects for orientation and delay, and significant interactions for orientation by gender, delay by distance, task by gender and orientation by distance by gender. 
- parallel cardinal _ linear (parallel cardinal)

- parallel oblique _linear (parallel oblique)

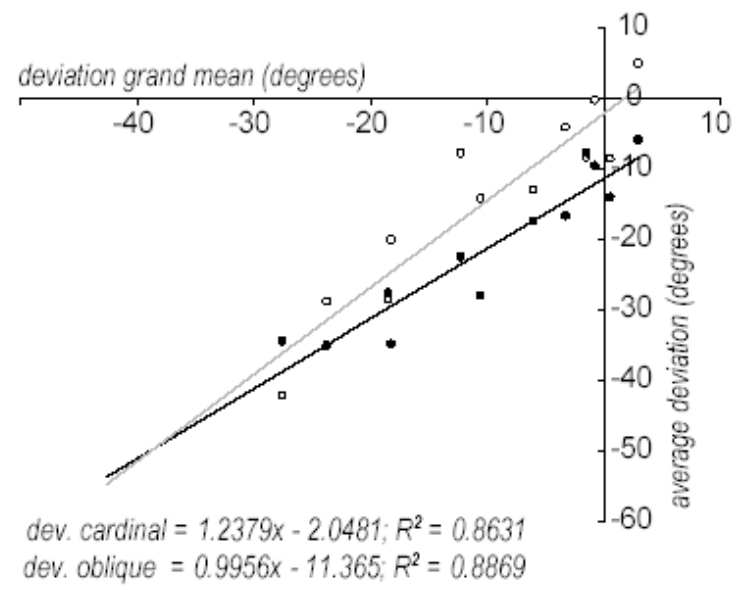

Figure 5. Each participant's average signed error for parallel matching of cardinal and oblique orientations at $60 \mathrm{~cm}$ intermanual distance, plotted against his/her overall average signed error, and the trend lines fitted through these data points.

\section{Task by gender}

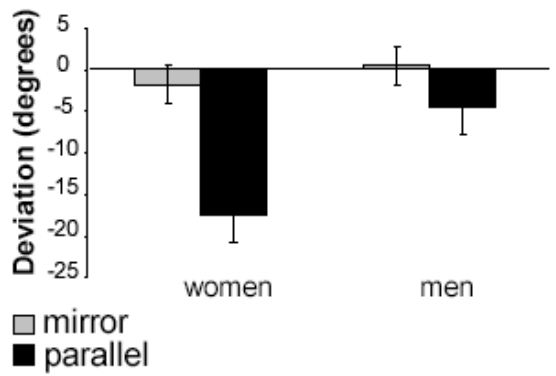

Figure 6. Mean signed error and standard error for each task and gender.

The main effect for orientation $(\mathrm{F}(5,50)=2.734, \mathrm{p}<0.029)$ resulted from a significantly lower variability for the 90 degree orientation, whereas the means for the other 5 orientations did not differ from each other. Orientation interacted with gender $(\mathrm{F} 5,50)=2.597, \mathrm{p}<0.036)$, in the sense that apart from the 90 degree orientation, the 0 degree orientation was also associated with lower variability in male participants. We found a main effect of delay $(F(2,20)=4.890, p<0.019)$, due to the fact that the short delay of $500 \mathrm{~ms}$ was associated with the lowest variability in the error, whereas the intermediate (5s) and long (10s) delay showed a similar, higher variability. The effect of delay interacted with distance $(\mathrm{F}(2,20)=3.896, \mathrm{p}<0.037)$, delay caused a greater increase in the variability at the largest $(60 \mathrm{~cm})$ distance. Although there was no main 
effect for task, there was a task by gender interaction $(F(1,10)=17.523, \mathrm{p}<0.002)$ : while variability was approximately equal in males and females for the parallel task, men showed a slight decrease in variability for the mirror task, whereas women showed a slight increase for the mirror task, compared to the parallel task. The interaction between orientation, distance and gender $(\mathrm{F}(5,50)=2.963, \mathrm{p}<0.02)$ was caused by the fact that men and women showed different orientation-dependent patterns of variability at the largest distance. Whereas the locations of minimum and maximum variability were very similar at $30 \mathrm{~cm}$ intermanual distance, the locations were shifted by approximately thirty degrees at the $60 \mathrm{~cm}$ distance. Men showed the lowest variability for the 0 and 60 degree orientations and the highest for 30 and 150 degree orientations, while women were most precise for 30 and 90 degree orientations and least precise for 0 and 60 degrees. Note that we did not find an oblique effect in the precision of the responses in analyses where we categorized orientations as oblique or cardinal (delay $\mathrm{x}$ task $\mathrm{x}$ distance $\mathrm{x}$ oblique $\mathrm{x}$ gender analysis: $\mathrm{F}(1,10)=0.061(\mathrm{p}<0.810)$ and task $\mathrm{x}$ distance $x$ oblique $x$ gender analysis: $F(1,10)=2.619(\mathrm{p}<0.137))$.

Although the 90 degree orientation was associated with the lowest variable error, we did not find an oblique effect when performing a delay (3) by task (2) by distance (2) by obliqueness (2) repeated measures GLM with gender as between factor, nor did we find an oblique by gender interaction.

\section{Discussion}

The present study examined the effect of instruction on haptic matching performance. Blindfolded participants were instructed to rotate a test bar with their right hand in such a way that it paralleled or mirrored the orientation of a reference bar, which could appear in different orientations and was felt with the left hand. Intermanual distance, i.e. distance between the reference and test bar, was either 30 or $60 \mathrm{~cm}$. In accordance with hypothesis 1 , we found an effect of Task: participants made significantly smaller errors in the mirror task compared to the parallel task. In addition, we also found a significant Task by Distance interaction. Parallel matching performance was characterized by large clockwise errors, which increased at greater intermanual distance and when oblique orientations had to be matched. Mirror matching performance induced small clockwise errors at $60 \mathrm{~cm}$, whereas it induced counter clockwise errors for some orientations at $30 \mathrm{~cm}$ distance between the bars. For the $90^{\circ}$ trials, we found a task effect at $60 \mathrm{~cm}$, but not at $30 \mathrm{~cm}$. In line with hypothesis 4 , orientation of the reference bar had a significant effect on error size. Orientation was found to interact with Task and with Distance. As hypothesized, there was a gender effect. Male participants outperformed female participants, an effect which was most prominent for parallel matching. Finally, Delay did not affect matching error.

\section{Effects of task, distance and orientation}

A significant task effect due to superior performance for mirror compared to parallel matching has previously been reported by Newport et al. (2002) and Kappers (2004). A performance difference between parallel versus mirror matching is especially striking if it occurs for orientations that require identical responses in both mirror and parallel 
task, i.e. at $0^{\circ}$ and $90^{\circ}$. At $0^{\circ}$, the participant still has the choice to position the hand with the thumb towards or away from the body. Thus, task effects on the $0^{\circ}$ trials might still be caused by motor-executive differences, meaning that participants might have preferred to put their hands in a mirrored position (both thumbs towards or away from the body) in the mirror task, and in a parallel configuration in the parallel task. However, a variation in the positioning of the hands would not affect the $90^{\circ}$ trials, where physical limitations ensure that participants will always have their thumbs pointing towards the midsagittal plane. Therefore, in the $90^{\circ}$ trials, any remaining difference in performance most likely reflects a cognitive-computational difference between the tasks. Interestingly, Kappers (2004) indeed reported differences between deviations in both tasks at $90^{\circ}$ at $120 \mathrm{~cm}$, although she did not statistically compare mirror and parallel matching at the $90^{\circ}$ orientation. The current study extends these results to distances within normal reaching range, reporting a significant task difference for $90^{\circ}$ trials at $60 \mathrm{~cm}$ distance.

The performance difference for identical trials in the mirror and parallel matching condition shows that our task instruction worked and that participants approached both tasks in different ways, and, more importantly, it suggests that participants used a different cognitive-computational strategy to solve both tasks, at least at $60 \mathrm{~cm}$. Adhering to the intermediate reference frame model proposed by Kappers (2004), the strategy difference could occur as a task-dependent weighting of the contributions of the allocentric Cartesian frame and the egocentric polar coordinate system fixed on the body symmetry plane. Kappers uses a similar explanation for inter-individual differences in parallel matching performance. The results from the present study, as well as other studies, indicate that reference frame weighting might also vary intra-individually, depending on task instruction (Kappers, 2004; Newport et al., 2002) and/or implicit task demands, such as the availability of (noninformative) visual input (Newport et al. 2002; Zuidhoek et al. 2004) and orienting towards the test hand (Zuidhoek et al., 2004). However, the intermediate reference frame model could also explain the difference between parallel and mirror matching at the $90^{\circ}$ orientation as a result of the opposing operations performed with respect to the reference line through ego centre and test bar pivot (see figure $1, \mathrm{~b}$ and $\mathrm{d}$ and $7, \mathrm{~b}$ and $\mathrm{d}$ ). To obtain the response in the parallel task, the orientation difference between the reference bar and the reference line through ego centre and reference bar pivot is subtracted from the orientation of reference line running through the test bar pivot, whereas it is added in the mirror task. Our finding that the task difference for the $90^{\circ}$ trials does not persist at $30 \mathrm{~cm}$ distance between the bars might indicate a strategy shift. Alternatively, it might be caused by a ceiling effect in the parallel matching errors, due to the fact that the detrimental effect of the egocentric reference frame diminishes at small distances between the hands.

In line with the results of the current study, Kappers (2003) and Zuidhoek et al. (2003) reported significantly better performance for parallel matching at smaller distances between the bars. In terms of the intermediate reference frame model (Kappers, 2003) the improvement can be interpreted to result from the fact that the virtual reference lines (figure 1) of the intermediate reference frame become more parallel to the Cartesian axes at smaller distances between the bars, reducing the discrepancy between what is perceived as parallel and what is truly parallel. In Kappers' intermediate reference frame model, all errors in haptic spatial matching arise from the egocentric reference 
frame. Since mirror matching can be performed without errors in the egocentric frame, mirror performance should not show any systematic errors and should be independent from the distance between the hands.

In addition, the intermediate reference frame model predicts that performance is independent from the orientation of the bars. However, a significant effect of orientation was found for mirror and parallel matching both in our study and in the study by Newport et al. (2002). Moreover, the direction of the errors was in some cases contrary to the direction of "pull" of the egocentric reference frame, especially at the shorter distance between the bars. At $30 \mathrm{~cm}$, counter clockwise average errors occurred in the mirror task for all orientations, except $120^{\circ}$ and $150^{\circ}$. In addition, mirror matching also resulted in counter clockwise errors for $30^{\circ}$ and $90^{\circ}$ trials at $60 \mathrm{~cm}$, and for $90^{\circ}$ trials in parallel matching at $30 \mathrm{~cm}$. Therefore, adjustments to the intermediate reference frame model might be necessary to describe these variations in error direction and orientation dependency. Although the use of egocentric forelimb based axes might account for the counter clockwise errors observed for the 90 degree orientation in parallel matching at $30 \mathrm{~cm}$, it cannot account for the fact that parallel matching of the other 5 orientations lead to opposite clockwise errors at the $30 \mathrm{~cm}$ distance, the reverse is true for the intermediate reference frame model.

The different error sizes for different orientations might reflect the influence of the asymmetric and anisotropic ability to rotate the hand, pulling responses towards the most natural orientation of the hand for a given arm configuration, in addition to a lower perceptual accuracy at extreme joint angles (Rossetti et al., 1994). The expected pattern of effects for parallel matching would be that orientations at increasingly counter clockwise distances from the most natural hand orientation would be encoded and reproduced with increasingly large clockwise deviations (and vice versa), on top of the general bias induced by the natural orientation of the hand. In general, the effect would be smaller in the mirror task, and would average to zero when the reference orientations are chosen in such a way that they are distributed equally over the range of all possible orientations. To some extent, this account can explain the general shape of the orientation dependency of the error in our data and in the data by Newport et al. (2002), as well as the interaction with distance, since the most natural orientation of the hand will vary at different positions with respect to the midsagittal plane. Nevertheless, it is in contradiction with the finding that the orientation effect does not average to zero in the mirror task, and with the results found by Kappers (2004), who does not report any significant systematic errors for mirror matching. Her mirror data show only small deviations in clockwise direction, for oblique orientations when the left hand was used for exploration of the reference bar and for cardinal orientations when the right hand was used for exploration. Apart from the inclusion of trials where the right hand was used for exploration of the reference bar, the most important difference in the study by Kappers (2004) is the use of a larger intermanual distance; being $120 \mathrm{~cm}$, whereas a distance of $20 \mathrm{~cm}$ in the study by Newport et al. (2002) and 30 and $60 \mathrm{~cm}$ in the present study. However, the rotational abilities of the hand affect all intermanual distances. Therefore, the orientation effect is most likely caused or moderated by another factor, dependent on intermanual distance and the direction of matching (whether the left or right hand is used for exploration). 
A factor that might be of importance to explain systematic errors in the mirror task is the positioning of the plane that the participant uses for the mirror operation with respect to the symmetry axes of the body, which coincided with the plane that orthogonally bisected the line between the pivots of the bars. If these planes are misaligned, systematic counter clockwise errors could arise in the mirror task. It can be derived that the overall effect of this shift is larger at small intermanual distances, because the relative discrepancy of the angles between the mirror plane and reference lines on the left and on the right becomes larger at smaller distances. This would be in agreement with the fact that the effect did not persist equally strong at the larger distance $(60 \mathrm{~cm})$ between reference and test bar, and has only been reported in the study by Newport et al. (2002) using short distances. The direction of the shift might depend on the matching direction (left hand exploration, right hand response). It might also reflect an asymmetry in spatial attention, i.e. an over-representation of the left hemispace, due to right-hemispheric dominance for spatial attention. For the parallel task, Zuidhoek et al. (2003) showed that direction of spatial attention had an effect on the error size. In our study, counter clockwise errors occurred mainly for orientations where the right hand pointed to the left.

The use of egocentric forelimb based axes might account for the counter clockwise errors observed for the 90 degree orientation in parallel matching at $30 \mathrm{~cm}$, which cannot be accounted for by the intermediate reference frame model. On the other hand, whereas the intermediate reference frame model can account for the fact that parallel matching of the other 5 orientations lead to opposite clockwise errors at the 30 $\mathrm{cm}$ distance, a model using egocentric forelimb axes could not. Both an egocentric forelimb model and the intermediate reference frame model fail to explain the systematic errors occurring in the mirror task, since both models predict error free mirror matching, because of their symmetry in the midsagittal plane of the body. Models which do not share this symmetry axis would be able to explain systematic errors in the mirror symmetry matching task. We therefore feel that it is worthwhile to include an example of such a model. Although speculative, we feel that the shifted midline model might be a plausible and elegant candidate, both from a mathematical and a neuropsychological perspective. Loosening the constraint that the centre of the reference frame should lay on the midsagittal plane of the body, it provides a one-parameter description of all phenomena explained by the intermediate reference frame model, extending it to the current observations for mirror matching in near peripersonal space.

The present study shows the need to incorporate the variability in error direction and size in models describing haptic matching for different stimulus orientations by including factors such as asymmetrical and anisotropic hand rotation as well as the potential effects of spatial attention. It might be that these factors can be accounted for by proper adjustments of the intermediate reference frame model. However, it might also be that a new model using different assumptions or a different level of description will prove more effective in the end.

\section{Oblique effect, gender and delay}

In accordance with previous studies (Gentaz and Hatwell, 1995; Kappers, 1999, 2002, 2003, 2004; Gentaz et al., 2001; Luyat et al., 2001; Luyat and Gentaz, 2002; Hermens and Gielen, 2003; van Mier et al., 2003; Fraussen et al., 2004; Fernandez and van Mier, 
2005) we found an oblique effect, in the sense that the average error for vertical and horizontal (cardinal) orientations was smaller than the average error for oblique orientations. In our view, the distinction between oblique and cardinal orientations is meaningful as long as there is evidence that the orientations joined in these categories evoke similar matching responses, and behave similarly across conditions. However, this was not the case in our data. There were orientations for which the error was close to zero, but these orientations were not necessarily cardinal. A change in the distance between the bars also changed the ranking order of the orientations with respect to the error. Most previous studies have tested at intermanual distances of $60 \mathrm{~cm}$ or more, using oblique orientations of 45 and 135 degrees, whereas we tested at 30 and $60 \mathrm{~cm}$, using $30,60,120$ and 150 degrees. We only found a special role for the 90 degree orientation, parallel to the body midline, independent of the distance between the bars and hands. For that reason, it may be important to keep in mind that the cardinal/oblique distinction is allocentrically defined. It seems very likely that what is experienced as oblique or cardinal is participant dependent, linked to participant-specific reference frames (Luyat et al., 2001; Kappers, 2004). Therefore, it may be more meaningful and informative to analyse separate orientations, instead of assuming oblique and cardinal clusters a-priori. We did not observe a reversal of the oblique effect for participants with large average errors as reported by Kappers (2003). Based on interpolation of the fitted linear trend lines, we expect that the oblique effect might reverse for potential participants with an average error over $40^{\circ}$ for the parallel task at $60 \mathrm{~cm}$ (across all orientations and both distances) in our study. Kappers (2003) reported a reversed oblique effect for participants with average errors of $57^{\circ}$ or higher. In their study, the distance between the bars $(120 \mathrm{~cm})$ was twice as big as our largest distance $(60 \mathrm{~cm})$, thus inducing larger errors. In addition, the oblique orientations $\left(45^{\circ}\right.$ and 135$)$ used by Kappers are different from ours.

The gender effect observed in our study is in line with gender differences previously reported in spatial tasks (Hammer et al., 1995; Voyer et al., 1995; Crucian and Berenbaum, 1998; Parsons et al., 2004) and more specific in haptic parallelity tasks for adults, adolescents and children (Kappers, 2003; Van Mier et al., 2003; Fernandez and van Mier, 2005). Although sighted and blind women were reported to have better tactile acuity than men (van Boven et al., 2000; Goldreich and Kanics, 2003), they apparently do not benefit from this perceptual advantage in macro geometric tasks in the somatosensory domain. The gender difference was most striking for parallel matching at $60 \mathrm{~cm}$, which might point out that women are more egocentrically biased than men.

A previous study by Zuidhoek et al. (2003) reported that a delay of 10 seconds reduced parallel matching error at 120 and $60 \mathrm{~cm}$ distances between reference and test bar. In our study, a 5 or $10 \mathrm{sec}$ delay did neither improve nor deteriorate matching error at either distance. This might be due to a ceiling effect at shorter distances, since the egocentric bias affecting parallel matching becomes smaller at smaller distances. It might also have to do with the mixed presentation of delays in the current study, given that Zuidhoek et al. (2003) used a blocked presentation of delays. The fact that delay did not result in a significant deterioration of performance, shows that memory traces and maintenance strategies for haptic stimuli were still effective at $10 \mathrm{sec}$ retention intervals, comparable to other tactile studies showing retention up to at least $15 \mathrm{sec}$ 
(Kiphart et al., 1992; Bowers et al., 1990; Burton and Sinclair, 2000; Woods et al., 2004).

\section{Precision effects}

In our view, the effects for the precision of the responses do not fundamentally change our interpretation of the data as described in the previous paragraphs. The effect of delay can be explained as increasing noise in the representation when stored in memory. The effect of orientation parallels the effects observed for the mean errors. There was no effect of task, indicating that the two tasks are probably executed in the same reference frame. The interaction between task and gender signalizes that men and women might use a different reference frame for the mirror task. Independent of task, intermanual distance has a differential effect of the orientation dependent pattern of precision for men and women. Women were always most precise for the 90 degree orientation, whereas men showed a shift at the largest distance. This might indicate that women have a stronger representation of the midsagittal plane of the body.

\section{Conclusion}

Greater errors were found for parallel versus mirror orientation matching, for oblique versus cardinal orientation matching, for large versus small distances between the bars and for female versus male participants. In addition, we found a significant difference between parallel and mirror matching at an intermanual distance of $60 \mathrm{~cm}$ for the $90^{\circ}$ orientation, an orientation that in principle required the same response in both tasks. This result clearly suggests an effect of task demand.

However, for mirror matching, we found counter clockwise errors at $30 \mathrm{~cm}$ distance between the bars. The direction of these errors is not in accordance with the view that haptico-spatial information is represented in a frame intermediate between an allocentric Euclidean frame and an egocentric polar frame, centred on the midsagittal plane. Based on these findings, we suggest that haptic performance in near-peripersonal space might be dominated by different mechanisms than those that play a role at distances over 60 centimetres.

Summarizing, the present study extends previous findings of systematic errors for haptic spatial matching, and, in addition, revealed unexpected effects at small distances from the body, distances that correspond to the natural dimensions of haptic workspace. The results provide additional constraints, necessary for the specification of an adequate mathematical and neuronal model of the processes involved in haptic spatial tasks.

\section{Acknowledgement}

We thank the participants and Kim Huiskamp for her help collecting the data. 


\section{References}

Apelle S (1972) Perception and discrimination as a function of stimulus orientation: the "oblique effect" in man and animals. Psychol Bull 78: 266-278.

Batista A (2002) Inner space: reference frames. Curr Biol 12: R380-383.

Bingham GP, Crowell JA, Todd JT. (2004) Distortions of distance and shape are not produced by a single continuous transformation of reach space. Percept Psychophys 66: 152-169.

Bowers RL, Mollenhauer MS, Luxford J (1990) Short-term memory for tactile and temporal stimuli in a shared-attention recall task. Percept Mot Skills 70: 903-913.

Burton H, Sinclair RJ (2000) Attending to and remembering tactile stimuli: a review of brain imaging data and single-neuron responses. J Clin Neurophysiol 17: 575-591.

Carrozzo M, Stratta F, McIntyre J, Lacquaniti F (2002) Cognitive allocentric representations of visual space shape pointing errors. Exp Brain Res 147: 426-436.

Crucian GP, Berenbaum SA (1998) Sex differences in right hemisphere tasks. Brain Cogn 36: 377-389.

Cuijpers RH, Kappers AM, Koenderink JJ (2000) Large systematic deviations in visual parallelism. Perception 29: 1467-1482.

Cuijpers RH, Kappers AM, Koenderink JJ (2003) The metrics of visual and haptic space based on paralellity judgements. J Math Psychol 47: 278-291.

Fernandez I, van Mier H. (2005) Haptic perception of paralellity in adolescents and adults. 14th conference of the European Society for Cognitive Psychology (ESCoP).

Flanders M, Soechting JF (1995) Frames of reference for hand orientation. J Cogn Neurosci, 7: 182-195.

Fraussen M, Kappers A, van Mier H (2004) Haptic perception of spatial relations in children and adults. Third Dutch Endo-Neuro-Psycho meeting, pp. 244.

Gentaz E, Hatwell Y (1995) The haptic oblique effect in children's and adults' perception of orientation. Perception, 24: 631-646.

Gentaz E, Hatwell Y (1998) The haptic oblique effect in the perception of rod orientation by blind adults. Percept Psychophys 60: 157-167.

Gentaz E, Luyat M, Cian C, Hatwell Y, Barraud PA, Raphel C (2001) The reproduction of vertical and oblique orientations in the visual, haptic, and somato- vestibular systems. Q J Exp Psychol A. 54: 513-526.

Gentaz E, Streri A. (2004) An "Oblique Effect" in infants' haptic perception of spatial orientations. J Cogn Neurosci 16: 253-259.

Goldreich D, Kanics IM (2003) Tactile acuity is enhanced in blindness. J Neurosci 23: 3439-3445.

Hammer RE, Hoffer N, King WL (1995) Relationships among gender, cognitive style, academic mayor, and performance on the Piaget water-level task. Perc Motor Skills 80: 771-778.

Henriques DY, Flanders M, Soechting JF (2004) Haptic synthesis of shapes and sequences. J Neurophysiol 91: 1808-1821.

Henriques DY, Flanders M, Soechting JF (2005) Distortions in the visual perception of shape. Exp Brain Res 160: 384-393.

Henriques DY, Soechting JF (2003) Bias and sensitivity in the haptic perception of geometry. Exp Brain Res 150: 95-108.

Hermens F, Gielen S (2003) Visual and haptic matching of perceived orientations of lines. Perception 32: 235-248.

Hermens F, Kappers AML, Gielen SCAM (2006). The structure of fronto-parallel haptic space is task dependent. Percept Psychophys 68: 62-75.

Hu Y, Goodale MA (2000) Grasping after a delay shifts size-scaling from absolute to relative metrics. J Cogn Neurosci 12: 856-868. 
Kaas AL, van Mier HI, Goebel R. The neural correlates of human working memory for haptically explored object orientations. Cereb Cortex. In Press.

Kappers AML (1999) Large systematic deviations in the haptic perception of parallelity. Perception 28: 1001-1012.

Kappers AML (2002) Haptic perception of parallelity in the midsagittal plane. Acta psychol 109: 25-40.

Kappers AML (2003) Large systematic deviations in a bimanual parallelity task: further analysis of contributing factors. Acta Psychol 114:131-145.

Kappers AML (2004) The contributions of egocentric and allocentric reference frames in haptic spatial tasks. Acta Psychol 117:333-340.

Kappers AML, Koenderink JJ (1999) Haptic perception of spatial relations. Perception 28:781-795.

Kiphart MJ, Hughes JL, Simmons JP, Cross HA (1992). Short-term haptic memory for complex objects. Bull Pyschon Soc 30: 212-214.

Luyat M, Gentaz E, Corte TR, Guerraz M (2001) Reference frames and haptic perception of orientation: body and head tilt effects on the oblique effect. Percept Psychophys 63: 541-554.

Luyat M, Gentaz E (2002) Body tilt effect on the reproduction of orientations: studies on the visual oblique effect and subjective orientations. J Exp Psychol Hum Percept Perform 28: 1002-1011.

McIntyre J, Stratta F, Droulez J, Lacquaniti F (2000) Analysis of pointing errors reveals properties of data representations and coordinate transformations within the central nervous system. Neural Comput 12: 2823-2855.

Newport R, Rabb B, Jackson SR (2002) Noninformative vision improves haptic spatial perception. Curr Biol 12: 1661-1664.

Oldfield, R.C. (1971) The assessment and analysis of handedness: the Edinburgh inventory. Neuropsychologia 9: 97-113.

Parsons TD, Larson P, Kratz K, Thiebaux M, Bluestein B, Buckwalter JG, Rizzo AA (2004) Sex differences in mental rotation and spatial rotation in a virtual environment. Neuropsychologia 42: 555-562.

Rossetti Y, Meckler C, Prablanc C (1994) Is there an optimal arm posture? Deterioration of finger localization precision and comfort sensation in extreme arm-joint postures. Exp Brain Res 99: 131-136.

Van Boven RW, Hamilton RH, Kauffman T, Keenan JP, Pascual-Leone A (2000) Tactile spatial resolution in blind Braille readers. Neurology 54: 2230-2236

Van Mier HI, Blommaert J, Kappers AM (2003) Haptic perception of parallelity in children and adults. Program No. 196.18. Society for Neuroscience.

Voyer D, Voyer S, Bryden MP (1995) Magnitude of sex differences in spatial abilities: a meta-analysis and consideration of critical variables. Psychol Bull 117: 250-270.

Woods AT, O'Modhrain S, Newell FN (2004) The effect of temporal delay and spatial differences on cross-modal object recognition. Cogn Affect Behav Neurosci 4: 260-269.

Zuidhoek S, Kappers AM, van der Lubbe RH, Postma A (2003) Delay improves performance on a haptic spatial matching task. Exp Brain Res 149: 320-330.

Zuidhoek S, Visser A, Bredero ME, Postma A (2004) Multisensory integration mechanisms in haptic space perception. Exp Brain Res 157: 265-268. 


\section{CHAPTER 3}

\section{The effect of visuo-haptic congruency on haptic spatial matching}




\begin{abstract}
Accurate eye-hand coordination is of crucial importance for everyday visuo-haptic object-manipulation. A previous study found a positive effect of non-informative vision on haptic parallel matching, in which the manually explored orientation of a reference bar is subsequently matched by turning a test bar to a parallel orientation. However, vision was detrimental to mirror matching, in which a haptic orientation is mirrored in the body symmetry-plane.

The current study aimed to investigate the systematic effect of specific visual information on haptic tasks, presenting congruent and parametrically varied incongruent visual stimulus orientations during the exploration phase of a delayed haptic parallel and mirror matching task.

We found that visual incongruent information parametrically modulated task performance only for the parallel task, depending on the size and direction of the incongruence. However, haptic parallel matching did not improve significantly and mirror matching deteriorated when only congruent visual information was provided throughout a run. These results clearly show that there is no effect of visual input per se, but that the effect of visual information on haptics depends on the type of haptic task, the stability of visuo-haptic correspondence and the visuo-haptic incongruency level. Our study demonstrates the flexible use of different strategies, either visual or somatosensory, in haptic tasks and their specific susceptibility to multi-sensory integration.
\end{abstract}




\section{The effect of visuo-haptic congruency on haptic spatial matching}

$\mathbf{T}$ he haptic sense allows us to skillfully interact with objects in our environment. Reaching towards an object, as well as grip scaling require accurate visuo-haptic transfer of spatial object information. Whereas the haptic (active tactile) sense exceeds the visual sense when it comes to the extraction of material object properties, it is less suited for the extraction of spatial information (Lederman and Klatzky, 2004).

Systematic errors occurred when blindfolded participants were asked to match the orientation of a manually explored reference bar by turning a test bar to a parallel orientation, using the contralateral hand (Kappers, 1999, 2003; Newport et al., 2002; Kaas and Van Mier, 2006). In principle, veridical performance on this parallel matching task can only be achieved in an allocentric reference frame, preserving the metric of extrapersonal space. Yet, error size and direction show a high correlation with the natural orientation of the hands at the bar locations. Error size increased with larger horizontal distances between reference and test bar. In addition, errors typically showed a centrifugal direction, that is, the far end of the test bars was turned too far away from the midsagittal plane (centrifugal error, see figure 4). This indicates that the haptic system cannot factor out the biasing effect of hand orientation (Kappers, 2004).

In contrast, a similar spatial task, haptic mirror matching, induced much smaller errors. In this task, participants turned a test bar to an orientation which was the mirror image of a reference orientation with respect to the midsagittal plane of the body (Kappers, 2004; Kaas and Van Mier, 2006; Newport et al., 2002). Kappers showed that the parallel and mirror matching performance can be described in a common reference frame. This so-called intermediate reference frame is a weighted average of an egocentric, hand-centered frame, and an allocentric, world-based frame, in which the position of an object is coded with respect to other objects. Although the tasks can be described within a common reference frame, an accuracy difference arises between parallel and mirror matching because in mirror matching, the biasing effect of hand orientation is opposite for both hands. Hence, while parallel matching is characterized by a systematic centrifugal bias, the biasing effect of hand orientation in mirror matching cancels out.

A recent imaging study did not find any differences in the neural substrates underlying parallel and mirror matching (Kaas et al., in press; chapter 5). This might indicate that 
mirror and parallel matching also share a common reference frame at the cortical level. In fact, similar sensorimotor, frontal and parieto-occiptital areas were found in the delay between haptic exploration for subsequent parallel or mirror matching. Comparable parieto-occipital areas were previously reported to support visual and tactile orientation discrimination (Zangaladze et al., 1999; Valyear et al., 2006).

Even though haptic parallel and mirror matching might be supported by the same brain areas and a common intermediate reference frame, Newport et al. (2002) found that these tasks were differentially affected by "non-informative" visual cues from the surrounding room. Whereas parallel matching improved when vision was added, mirror matching was unaffected or even deteriorated. In this study, the abundance of vertical and horizontal cues in the visual surroundings, together with the presence of a fixed haptic default orientation parallel to the midsagittal plane, might have provided a stable visuo-haptic reference frame, changing the strategies used for parallel and mirror matching in the visual condition. Newport et al. interpreted this finding as evidence for a preference for the use of extrinsic, visual coordinates in the parallel task, and intrinsic, limb-based coordinates for the mirror task.

Klatzky et al. (1987) have shown that haptic sorting strategies change when visual input is available. Other studies have shown the importance of vision for motor planning and the calibration of somatosensation. Rock and Victor (1964) showed that haptic perception of shape was changed in participants wearing minifying lenses. In addition, it has been shown that the felt finger position is recalibrated according to the retinal position in prism adaptation studies (Clower and Boussaoud, 2000), that visual context shapes pointing errors (Battaglia-Mayer et al., 2003), and that the transformation between eye- and hand-centered reach locations is achieved in an eye-centered reference frame (Buneo et al., 2002). Finally, in a review, Shimojo and Shams (2001) describe a series of classic experiments in which they show how uncertainty in one modality is resolved by using temporally contingent information from other modalities.

The aim of the current study was to investigate the effect of visual (in)congruent orientation cues in the exploration phase of haptic parallel and mirror matching tasks. We assumed that parallel matching favors the use of visual coordinates and mirror matching prefers a somatosensory frame. Our first hypothesis was that the addition of congruent visual orientation cues during the exploration phase of a delayed haptic parallel task would improve performance, whereas the mirror accuracy would be unaffected or reduced (H1a and H1b). Secondly, we hypothesized that parallel matching accuracy would be parametrically reduced for the visual incongruent orientations compared to the congruent condition ( $\mathrm{H} 2 \mathrm{a}$ ), but better compared to the purely haptic condition (H3a). Mirror accuracy, on the other hand, was not expected to be parametrically dependent on visuo-haptic incongruence level (H2b), but generally reduced compared to the blindfolded condition (H3b). These hypotheses were tested by comparing visuohaptic and purely haptic (blindfolded) runs for both tasks. In the visuo-haptic runs exploration and matching was performed with contralateral hands. In the haptic part of the experiment, half of the runs was performed contralaterally, and the other half was performed ipsilaterally, i.e. using the same hand for exploration and matching. This allowed us to compare the orders of magnitude of the effect of vision and the effects of haptic spatial and intermanual transformations, and to make a comparison between the performace of each hand. Previous studies of haptic working memory 
have shown accurate maintenance across delays of up to 30 seconds (Burton and Sinclair, 2000; Kaas and Van Mier, 2006), and we therefore expected ipsilateral parallel matching, that is, simple replication of the explored orientation, to reveal errors close to zero. We tested the hypothesis that the errors in the ipsilateral parallel task would be comparable in size and direction to the errors in the contralateral mirror task, in which the orientation can be replicated by adopting a homologous posture with the contralateral hand (H4a). The effect of spatial transformation of a haptic orientation was tested in an ipsilateral mirror matching condition, in which the mirror operation was to be performed in the plane parallel to the midsagittal plane, through the center of the turn bar. This removes the possibility of using a purely hand centered matching strategy and also eliminates the advantage that the egocentric bias cancels out, as in contralateral mirror matching. Therefore, we expected to find that ipsilateral mirror matching would show errors with a similar direction and size closer to the errors in contralateral parallel matching (H4b). Finally, we explored the effect of exploration hand on matching accuracy, asking half of the participants to perform exploration with their left hand, while the other half used the right hand. Previous studies reported a slight advantage for the left hand (right hemisphere) on haptic tasks involving spatial features (Dodds, 1978; Minami et al., 1994).

\section{Materials and Methods}

\section{Participants}

Thirty right-handed participants (6 males and 24 females, average age 21.9 years, sd 2.6) were recruited through advertisements posted in several University buildings. Handedness was assessed with a Dutch translation of the Edinburgh Handedness Inventory (Oldfield, 1971). Participants had either normal or corrected-to-normal visual acuity. They were naive with respect to the purpose of the study, and were rewarded with a gift certificate or credits to fulfill their course requirements for participating in the experiment. The study was approved by the local ethics committee. Prior to the experiment, participants were naïve with respect to experimental goals and setup. Before starting the experiment, they received training on the different tasks. It was not until they fully understood the entire procedure that the actual experiment began.

\section{Apparatus}

The setup consisted of two turn bars $(10 \times 1 \mathrm{~cm})$ with a blunt plastic needle at each end, mounted on a large table. The bars' centers were $60 \mathrm{~cm}$ apart and positioned positioned at $11.5 \mathrm{~cm}$ from the edge (of the participant's side) of the table. The orientation of the bars could be read with an accuracy of 0.5 degrees. In the following, the bar used to present the reference orientation will be referred to as "reference bar", and the bar which participants had to turn to a matching orientation will be called "test bar". A large button box was removed directly behind the test bar (see figure 1a). Two small round patches of cloth were attached near the edge of the table, just in front of the bars' centers, indicating the resting positions for the fingers when partici- 
pants were not touching the bars. The hands or belly did not touch the edges of the table.

Participants did not receive any direct visual information from the two turn bars. A black vertical board (height $75 \mathrm{~cm} \mathrm{x}$ width $90 \mathrm{~cm}$ ) attached to the table top right in front of the participant, prevented any visual information of the bars and the hands. The board contained two square indentations (width $25 \mathrm{~cm}$, height $15 \mathrm{~cm}$ ) for the hands in the lower left and right corners, allowing participants to comfortably touch the turn bars without seeing their hands. Another circular opening (diameter $20 \mathrm{~cm}$ ), was located in the middle of the board, its center $50 \mathrm{~cm}$ above the table top. A black tube (diameter $20 \mathrm{~cm}$ x length $50 \mathrm{~cm}$ ) was attached with one end to the opening in the board and the other end to a computer screen (40 cm diagonal). This ensured that the participant could only see the screen itself, and would not be influenced by the edges of the computer screen or other external cues. Participants were seated on a chair with adjustable height, making sure that each participant was able to view the screen in a comfortable way. A black curtain, glued to the top of the board was placed over the participant's head and back, eliminating all visual input except for the stimuli presented on the computer screen (see figure 1b). Auditory instruction cues were presented to the participants through a set of headphones.
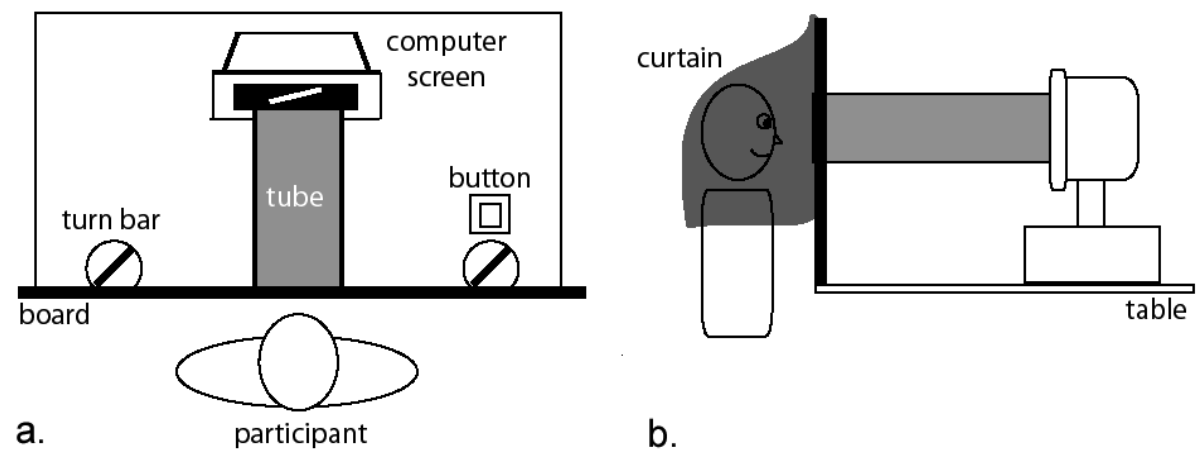

Figure 1. A schematic overview of the experimental set-up, a: seen from above and b: from aside. Participants were blindfolded in the haptic runs. The curtain used in the visuo-haptic runs is displayed as transparent grey in the picture. However, it was black and opaque in reality.

\section{Procedure}

Participants were sitting in front of the table supporting the two turn bars, their midsagittal plane coinciding with the centre plane of the table, halfway between the bars. An auditory cue $(1000 \mathrm{~Hz})$ indicated that participant's had to feel the orientation of the reference bar, in a time interval corresponding to the length of the tone $(1500 \mathrm{~ms}$; see figure 2). A visual stimulus was presented in the same time interval. Half of the participants used the left hand for exploration, and the other half the right hand. When the tone ended, the visual stimulus was removed, and participants returned their fingers to the resting position on a piece of cloth in front of the bar. After a delay of $5000 \mathrm{~ms}$, another tone $(2000 \mathrm{~Hz}, 100 \mathrm{~ms})$ announced that participants had to turn the test bar, either to a parallel or a mirrored version of the reference orientation, depending on the 
task instruction. The trial ended when participants pressed the response button behind the test bar. They were instructed to respond as quickly and accurately as possible. After finishing the trial, participants returned their fingers to the resting position, on the round patches of cloth in front of the bar. The inter trial interval (ITI) lasted for $6000 \mathrm{~ms}$, during which the experimenters wrote down the orientation of the test bar and prepared the reference orientation for the next trial.

The experiment consisted of a haptic and a visuo-haptic part. The visuo-haptic part of the experiment contained only contralateral runs of both parallel and mirror matching, with either congruent or incongruent visual feedback. This resulted in the following four visuo-haptic runs: bimanual parallel congruent, bimanual mirror congruent, bimanual parallel incongruent, and bimanual mirror incongruent. In the haptic part, participants were blindfolded, and completed four different runs: an ipsilateral parallel, ipsilateral mirror, contralateral parallel and contralateral mirror run. In the ipsilateral runs, the reference bar and test bar were explored and turned by the same hand, at the same location, in the contralateral runs with the contralateral hand, at $60 \mathrm{~cm}$ distance. The use of left and right hand was varied across participants. The order of visuo-haptic and haptic parts and the order of the runs within each part were counterbalanced across participants. Trials were presented in pseudorandom order.

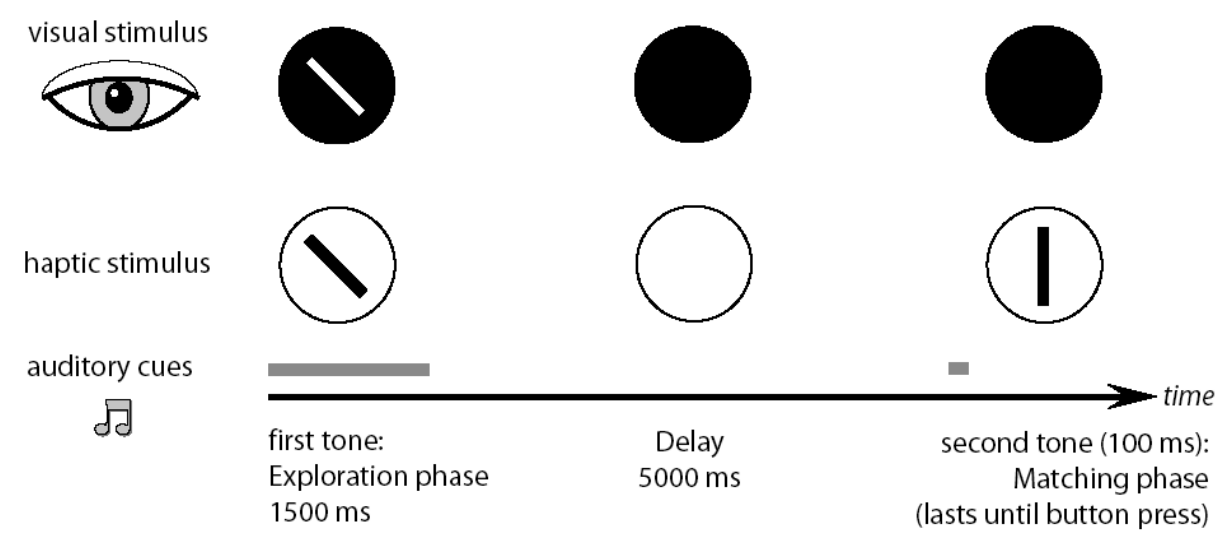

Figure 2. A sketch of the stimulus timing. The period during which the participant heard the auditory cues is indicated by a grey bar. In the visuo-haptic runs, visual input was added during the $1.5 \mathrm{~s}$ haptic exploration phase.

\section{Haptic stimuli}

To avoid standard 'clock time' orientations allowing the use of simple mnemonic strategies, twelve different orientations were used for the reference bar, within 10 degrees from 0, 45, 90 and 135 degrees (i.e. -10, 0, 10; 35, 45 55; 80, 90, 100; 125, 135, 145). The midsagittal plane of the participant's body was parallel to the 90 degree orientation. The orientation of the test bar was varied between 0 and 90 degrees, making sure that, within each run, the average turning distance was the same for all four reference orientations. Participants performed 12 (reference orientation) x 2 (tasks) x 2 (ipsi-contralateral) $\times 2$ (default orientation) $=96$ haptic trials, 24 trials per run. 


\section{Visual stimuli}

Visual stimuli consisted of a white stripe $(10 \mathrm{x} 1 \mathrm{~cm}$; visual angle approximately 10 degrees) on a black background, presented on a 15 inch computer screen (screen resolution $1024 \mathrm{x}$ 768). In the congruent runs, the visual orientations could be transformed into haptic orientations by a translation, followed by a single rotation in the midsagittal plane (90 degrees). The transformation matrix for this operation was invariant for all congruent trials. In the incongruent condition, visual stimuli differed from the haptic reference by an additional rotation in the frontoparallel plane of 16 or 8 degrees in either clockwise or counterclockwise direction, varying across trials (see figure 3). A pilot showed that participants were only able to visually discriminate orientation differences of 8 degrees or more.

Participants performed 12 (reference orientations) $\times 2$ (task) $\times 2$ (default) $=48$ trials in the two congruent visuo-haptic runs, and 12 (reference orientations) x 2 (task) x 4 (visual incongruence) $=96$ trials in the two incongruent runs. In the incongruent runs, the default orientation was varied in such a way that the average turning distance to the correct parallel or mirrored orientation was constant for each reference orientation and level of incongruence (8 or 16 degrees difference) within each run.

\section{Haptic stimulus}
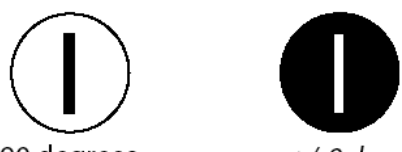

+/-O deg

Congruent

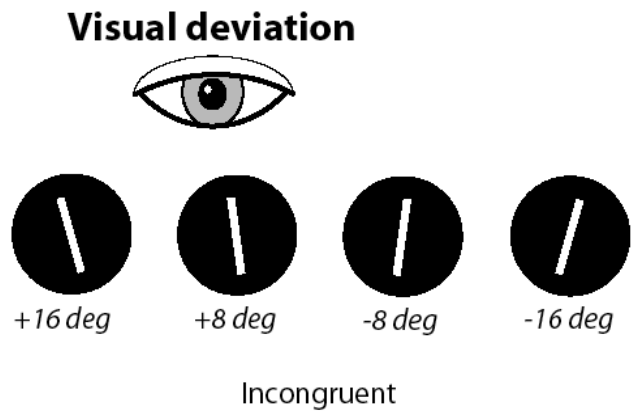

Figure 3. An example of the different visual stimuli presented during exploration of a particular haptic orientation in the visuo-haptic congruent ( 0 degree deviation) and incongruent $(+/-8$ and $+/-16$ degree deviation) runs.

\section{Data analysis}

Signed errors were calculated for every trial by subtracting the orientation of the reference bar from the orientation of the test bar. In the mirror condition, matching errors were expressed as deviations from the mirrored reference orientation (i.e. 60 degrees $=>120$ degrees). Errors were subsequently normalized to lie between -89 and +90 degrees and averaged within conditions. The sign was adjusted in such a way that a negative sign always corresponded to a centrifugal error direction, i.e. hands turned too far away from the body's midsagittal plane, whereas a positive error corresponded to a centripetal error direction, i.e. hands turned too far towards the midsagittal plane (see figure 4). 
Three repeated-measures ANOVAs were performed on the signed matching errors and the matching times separately, each with exploration hand as between-subject factor. The first repeated-measures ANOVA evaluated all runs together, with task (parallel or mirror) and condition (ipsilateral, contralateral, visual congruent, incongruent 8 degree, incongruent 16 degree) as within-subjects factors. Subsequently, a repeated-measures ANOVA was performed on the haptic runs, including within-subjects factors task (parallel or mirror) and haptic matching condition (ipsilateral or contralateral hand). A last repeated-measures ANOVA on the visuo-haptic incongruent runs included task and incongruence level (16 centrifugal, 8 centrifugal, 8 centripetal and 16 centripetal) as within-subjects factors, testing the potential systematic variation due to incongruence level $(\mathrm{H} 2 \mathrm{a}$ and $2 \mathrm{~b})$. Incongruence direction was defined with respect to the response hand; that is, trials with a visual deviation of 8 degrees clockwise were assigned to the 8 degree centripetal condition for participants responding with the left hand and to the 8 degree centrifugal condition for participants responding with the right hand, etc. Finally, hypotheses 1, 3, and 4 were directly tested with 6 paired t-tests, using a Bonferroni corrected alpha-level of $0.05 / 6=0.008$.

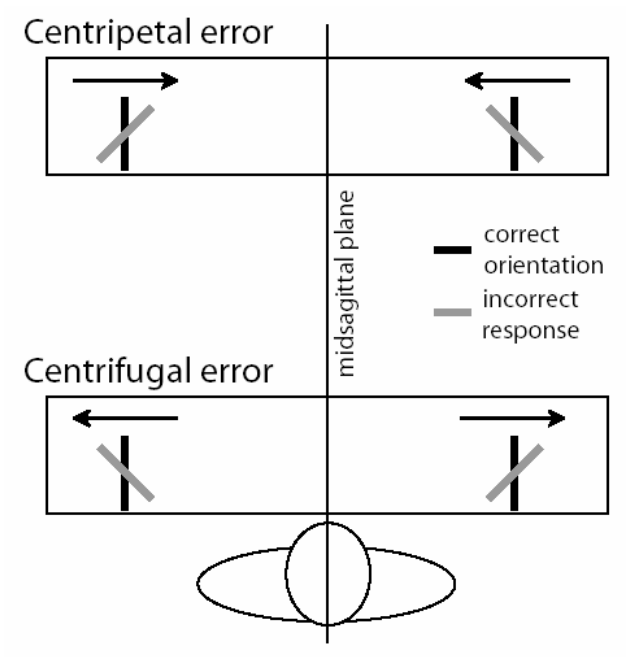

Figure 4. Centripetal errors occur when the test bar (grey line) deviates from the correct target orientation (black line) because the hand was turned too far towards the midsagittal plane, whereas centrifugal errors result from turning the hand too far away from the midsagittal plane.

\section{Results}

\section{Analysis on all runs}

Matching error Due to missing values, data of 1 participant from the right-hand exploration group had to be disregarded in this analysis. The condition (5) by task (2) by exploration hand (2) repeated-measures ANOVA on the signed matching errors showed a main effect for condition and task $(\mathrm{F}(2.40,67.10)=6.86$, Greenhouse-Geisser corrected for non-sphericity, $\mathrm{p}<0.05 ; \mathrm{F}(1,28)=69.67, \mathrm{p}<0.05)$, and an interaction between the two $(\mathrm{F}(2.63,73.63)=28.53 ; \mathrm{p}<0.05)$. The effect of condition was caused by signifi- 
cant differences between ipsilateral haptic matching and the contralateral visual congruent haptic matching, visual congruent and 8 degree incongruent haptic matching, and 8 degree incongruent and 16 degree incongruent haptic matching (within-subjects contrast $\mathrm{F}(1,28)=14.77 ; \mathrm{F}(1,28)=19.38 ; \mathrm{F}(1,28)=18.59 ; \mathrm{p}<0.05)$. The interaction effect was due to the opposite effects of ipsi- and contralateral task performance on the accuracy of mirror and parallel matching (within-subjects contrast: $F(1,28)=41.12$; $p<0.05$; see figure 5). The decreased accuracy for mirror matching in the congruent visual condition, as opposed to the slight improvement in parallel matching performance in the visual congruent condition also contributed (within-subjects contrast: $F(1,28)=28.54$; $\mathrm{p}<0.05)$. In addition, parallel matching accuracy decreased for 16 degree visuo-haptic incongruent condition compared to 8 degree incongruent, whereas accuracy on the mirror task was unaffected by this change in visual information (within-subjects contrast: $\mathrm{F}(1,28)=12.39 ; \mathrm{p}<0.05)$. When performing a separate repeated measures ANOVA on the haptic parallel task only, we found no significant difference between the contralateral blindfolded and visual congruent condition, and a trend for higher accuracy on the blindfolded versus 8 degree incongruent contrast $(F(1,28)=2.95$; $\mathrm{p}=0.097)$.

Matching time The repeated-measures ANOVA with condition and task as within- subjects factors and exploration hand as between-subjects factor, revealed a main effect of condition $(\mathrm{F}(2.49,69.58)=10.41$, Greenhouse-Geisser corrected for non-sphericity; $\mathrm{p}<0.05)$. The main effect of condition was due to increasingly slow matching times for the three visuo-haptic conditions compared to contralateral haptic matching (congruent: $\mathrm{F}(1,28)=12.76 ; 8$ degree incongruent: $\mathrm{F}(1,28)=18.18 ; 16$ degree incongruent: $\mathrm{F}(1,28)=11.66 ; \mathrm{p}<0.05$; see figure 5).

\section{Task by Condition}
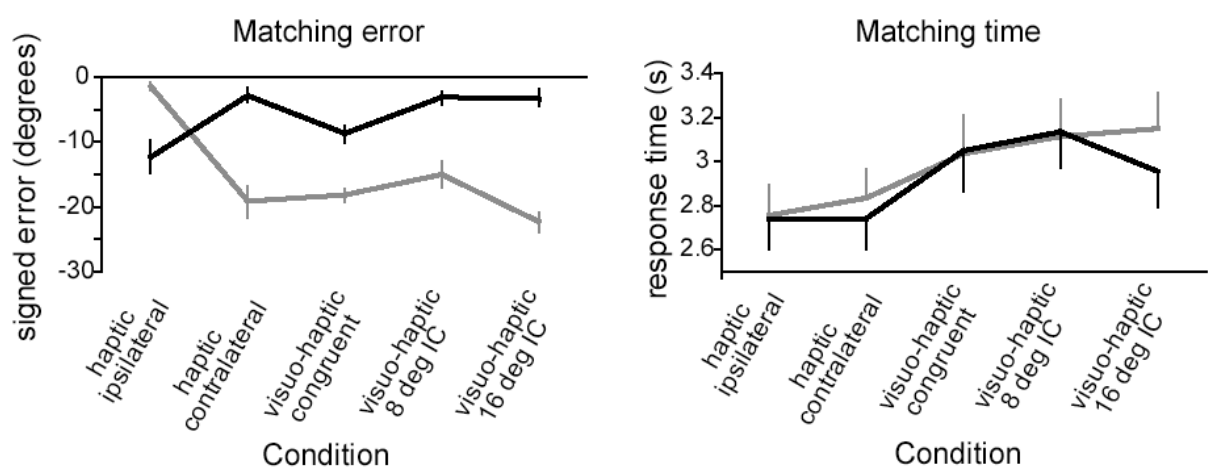

$$
\begin{aligned}
& \text { - parallel task } \\
& \text { — mirror task }
\end{aligned}
$$

Figure 5. Average signed matching errors and matching times, for each condition and task. The task by condition interaction in the matching errors was caused by opposite effects for ipsi- versus contralateral matching in the parallel and mirror tasks, by decreased accuracy for the mirror task with congruent visual information and decreased parallel matching accuracy in the 16 degree incongruent condition. Matching times became increasingly slow when adding visual congruent, 8 degree incongruent and 16 degree incongruent information. 


\section{The effects of matching condition and task on blindfolded haptic matching performance}

Matching error The task by matching condition by exploration hand analysis on the errors, revealed a significant interaction between task and matching condition $(\mathrm{F}(1,28)=41,12 ; \mathrm{p}<0.05)$. While ipsilateral parallel matching resulted in very small errors, contralateral parallel matching showed much larger errors in centrifugal direction (see figure 6). This pattern was reversed for mirror matching. There were trends for an effect of both task and matching condition $(F(1,28)=3.63 ; p=0.07$ and $F(1,28)=3.59$; $\mathrm{p}=0.07)$. On average, the parallel task resulted in larger errors $(-10)$ than the mirror task (-8). The matching-hand trend was caused by the larger centrifugal errors for contralateral (-11) versus ipsilateral matching $(-7)$.

Matching time The task by matching condition repeated-measures ANOVA with exploration hand as between-subjects factor showed a significant interaction effect for exploration hand and matching condition $(\mathrm{F}(1,28)=4.85, \mathrm{p}<0.05)$. For the group using the left hand for exploration, matching times did not significantly change when the ipsilateral (left) or contralateral (right) hand was used for the matching. The group using the right hand for exploration was faster when the same hand was used for the matching. When analyzing the left and right hand groups separately, no significant effects of matching and task were found for the left hand, but the right hand showed a trend for faster mirror matching $(\mathrm{F}(1,14)=4.45 ; \mathrm{p}=0.053)$, and faster ipsilateral matching $(\mathrm{F}(1,14)=3,97 ; \mathrm{p}=0.066$; see figure 6$)$.

\section{The effect of visuo-haptic incongruence direction}

Matching error The task (2) by incongruence level (4) by exploration hand (2) analysis on the signed error revealed significant main effects for task and incongruence $(\mathrm{F}(1,28)=65.09, \mathrm{p}<0.05 ; \mathrm{F}(2.35,84)=24.78$, Greenhouse-Geisser corrected for nonsphericity, $\mathrm{p}<0.05)$, a significant interaction between task and incongruence level $(\mathrm{F}(3,84)=13.85 ; \mathrm{p}<0.05$; figure 7$)$, and a trend for an interaction between incongruence level and exploration hand $(\mathrm{F}(2.35,84)=2.64$, Greenhouse-Geisser corrected for nonsphericity; $\mathrm{p}=0.070$ or $\mathrm{F}(3,84)=2.64$, uncorrected; $\mathrm{p}=0.055$; see figure 8 ). As indicated by figure 7 , incongruence level did not affect mirror task performance, whereas the parallel task showed a reduced centrifugal error size for centripetal compared to centrifugal incongruent visual information. The lowest parallel matching error occurred when an 8 degrees centripetal visual stimulus was shown at stimulus exploration. The improvement was slightly stronger for left than right hand exploration (repeated contrast for task by incongruence by exploration hand: $F(1,28)=4.77 ; \mathrm{p}<0.05)$, as shown in figure 8 . When directly comparing parallel matching accuracy for the different incongruence levels to the purely haptic parallel matching condition (in a repeated measures ANOVA with information as within- and exploration hand as between factor; see dotted line in figure 8), we found that the 16 degree centrifugal level significantly decreased parallel matching accuracy $(\mathrm{F}(1,28)=14.06 ; \mathrm{p}<0.05)$, while the 8 degree centripetal level of visual incongruence significantly improved parallel matching accuracy $(\mathrm{F}(1,28)=12.38 ; \mathrm{p}<0.05$; Note that the direction of visual deviation was defined with respect to the exploration hand. A centrifugal direction indicates that if the exploration hand would be made parallel to the visual orientation, it would be turned too far away 
from the body's midsagittal plane, whereas the reverse is true for the centripetal deviation). In contrast, the mirror task remained unaffected by the visual information.

Matching time The task (2) by incongruence (4) by exploration hand (2)analysis only revealed a significant effect of task on matching time $(F(1,28)=7.05 ; \mathrm{p}<0.05)$. Parallel matching was performed significantly slower than mirror matching (3.2 seconds versus 3.1 seconds on average).

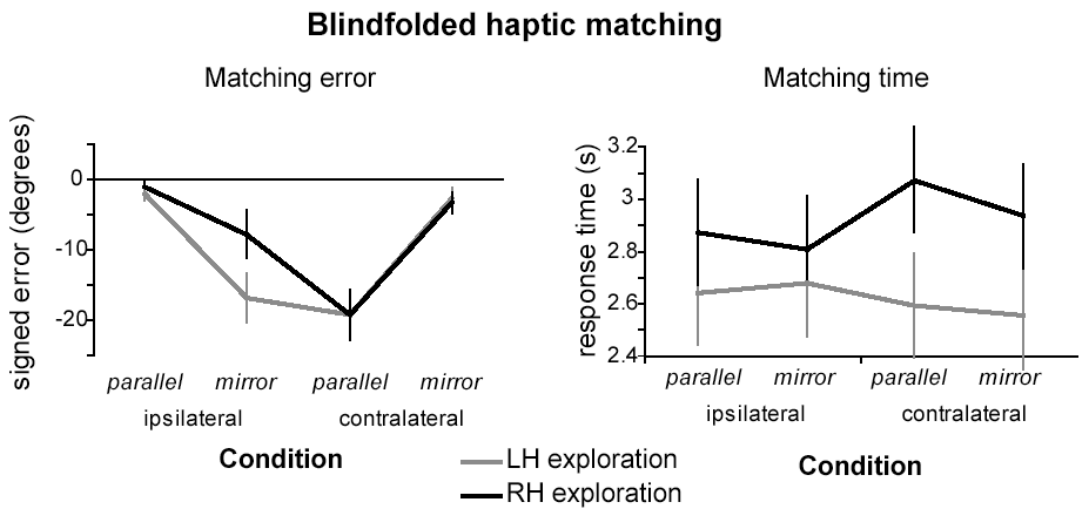

Figure 6. Average signed matching errors and matching times from the (blindfolded) haptic matching runs.

\section{Task by incongruence level}

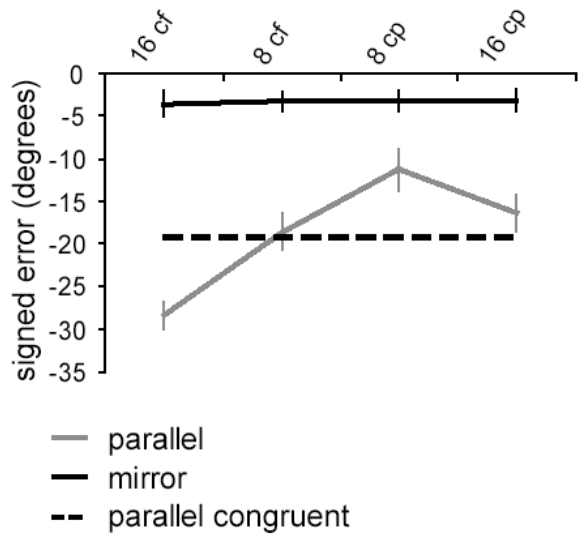

Figure 7. The parallel and mirror task were differentially affected by the levels of visuo-haptic incongruence (cf $=$ centrifugal, $\mathrm{cp}=$ centripetal). The average parallel matching error in the visuo-haptic congruent condition is also plotted for comparison (dotted line). 


\section{Exploration hand by Incongruence}

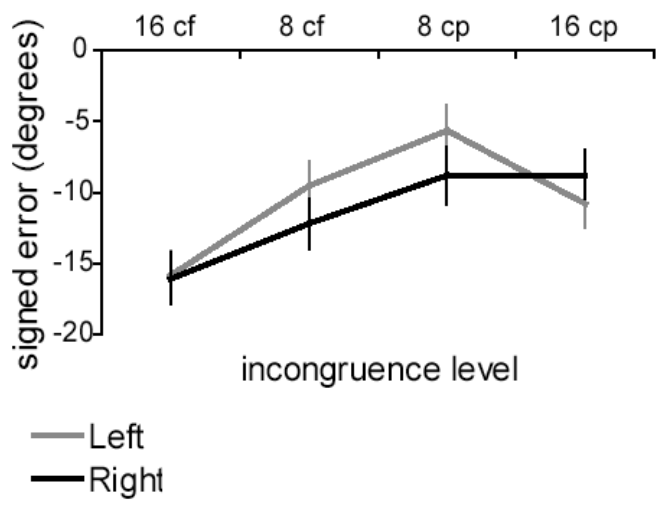

Figure 8. Average signed matching errors for the different levels of visuo-haptic incongruence $(\mathrm{cf}=\mathrm{cen}-$ trifugal, $\mathrm{cp}=$ centripetal), for left hand $(\mathrm{LH})$ and right hand $(\mathrm{RH})$ exploration.

\section{Paired t-tests on matching error hypotheses 1, 3 and 4}

Matching error Hypothesis 1a, concerning the positive effect of visuo-haptic congruence on haptic matching was rejected. Visuo-haptic congruence did not improve haptic parallel matching compared to the blindfolded contralateral condition $(t(29)=0.37$; $\mathrm{p}=0.72$ ). Hypothesis $1 \mathrm{~b}$ was not rejected, haptic mirror accuracy was reduced in the presence of visual congruent information $(\mathrm{t}(29)=-5.33 ; \mathrm{p}<0.0005)$. Hypothesis $3 \mathrm{a}$, stating an overall positive effect of the visuo-haptic incongruent information on parallel matching compared to the blindfolded contralateral condition was rejected: there was no accuracy difference between these conditions $(t(29)=0.24 ; \mathrm{p}=0.81)$. Hypothesis $3 \mathrm{~b}$, stating that there would be a lower or equal accuracy for the incongruent mirror condition compared to the blindfolded mirror condition was not rejected; the accuracy did not significantly differ between these conditions $(t(29)=0.50 ; p=0.62)$. Finally, both hypothesis $4 \mathrm{a}$ and $4 \mathrm{~b}$ were not rejected. The errors in haptic ipsilateral parallel matching were similar to the errors in haptic contralateral mirror matching $(t(29)=1.15$; $\mathrm{p}=0.26$ ), and, although showing a trend for being smaller in size, the errors in haptic ipsilateral mirror matching were not significantly different from those found in haptic contralateral parallel matching $(\mathrm{t}(29)=1.95 ; \mathrm{p}=0.061)$.

\section{Discussion}

The main objective of our study was to investigate the effect of visual orientation information on delayed haptic orientation matching. We provided congruent and parametrically varied incongruent visual information during the exploration phase of a haptic parallel and mirror task. In line with our hypotheses, we found that the accuracy of parallel matching parametrically varied with the level and direction of incongruent visual information (H2a). While centrifugal visual information (with an incongruence 
direction compatible with the direction of the egocentric bias) decreased parallel matching accuracy, a moderately centripetal visual deviation improved parallel matching accuracy. In contrast, mirror matching accuracy was unaffected in runs with incongruent visual information ( $\mathrm{H} 2 \mathrm{~b}$ and $\mathrm{H} 3 \mathrm{~b}$ ), while it was even reduced during runs with only congruent visual information (H1b). Contrary to our hypothesis, there was no general beneficial effect of visual information on parallel matching accuracy, neither for congruent visual runs (H1a), nor for incongruent visual runs (H3a).

\section{The effect of exploration hand and of ipsilateral/contralateral haptic matching}

In addition to the effect of visuo-haptic incongruence, we investigated the accuracy of delayed (blindfolded) haptic matching using the same hand or different hands for exploration and matching. In line with our expectation, we found that the error for the replication (i.e. ipsilateral parallel matching) of a haptically explored orientation after a delay of five seconds was close to zero, demonstrating the accuracy of the haptic sense and the efficiency of haptic working memory. In addition, the accuracy for the replication of a haptically explored orientation with the same hand (ipsilateral parallel matching) did not significantly differ from the accuracy of replication with the contralateral hand (contralateral mirror matching; $\mathrm{H} 4 \mathrm{a}$ ). When haptic matching required a spatial transformation, there was no significant difference in the errors for ipsilateral compared to contralateral haptic matching (ipsilateral mirror vs. contralateral parallel task; H4b), although the errors for ipsilateral mirror matching were smaller.

Exploring the effect of hand used for exploration, we found faster matching times for the left hand. Right hand matching times were modulated by matching condition and task. Matching times were faster for right hand contralateral mirror matching versus parallel matching, and right hand ipsilateral versus contralateral matching. Both ipsilateral matching and contralateral mirror matching might depend more on sensorimotor skills, while contralateral parallel matching requires an abstract spatial representation. Therefore, the generally increased matching times for right hand matching, and for contralateral parallel matching in particular, suggest the recruitment of ipsilateral resources in the right hemisphere in these conditions. This would be in accordance with the view that the left hand (i.e. right hemisphere) is dominant for spatial tasks (Smith et al., 1977; Dodds, 1978; Minami et al., 1994). However, since the matching time measure is fairly indirect and noisy, this interpretation has to be treated with caution.

\section{The appropriateness of congruent visual information for parallel and mirror matching}

Our results clearly show that the effect of visual information depends on the type of haptic task and the level of congruency of the visual information. Congruent visual information was detrimental to the mirror task, which allowed a purely somatosensory strategy. Incongruent visual information had no effect on this task, but did affect the parallel task, which requires an allocentric reference frame for veridical performance.

The visual and haptic orientations in the current study were presented at different locations, in different spatial planes. In the congruent runs, the spatial transformation to establish the congruence of the visual and haptic orientation was stable, and in accordance with proprioceptive information on the position of the head and eyes with respect to the hand. Therefore, in both the parallel and the mirror task, the congruence 
might be established more or less automatically and reliably. This might bias the weighting of visual and haptic information in favor of the visual modality and consequently, the strategy used for the mirror task. The increased matching times for mirror matching in the visual congruent runs, equivalent to those for parallel matching, might support the view that a different, less somatosensory matching strategy is used in the matching phase. However, due to the intrinsic egocentric bias in converting orientations in haptic space to an allocentric representation, the attempt to integrate the visual information causes greater errors for the mirror task in congruent runs.

In the incongruent runs, the visual and haptic information are not proprioceptively congruent. An additional rotation in the plane of the visual or haptic bar is needed to equate the visual and haptic orientation. A similar rotation would also be required in blindfolded contralateral parallel matching to factor out the difference in the orientation of the hands at different locations. Such an extra processing step is not needed for accurate mirror performance, and therefore, the less computationally demanding somatosensory strategy might prevail, resulting in the active blocking of visual information on this task. This is exemplified by the performance level, which was comparable to the level for blindfolded mirror matching. The increased matching times most likely reflect the active inhibition of the visual matching strategy, which is easier for trials with higher visuo-haptic incongruence. In contrast, the visual incongruent information did affect performance in the parallel task, depending on the judged congruence, i.e. the size of the visuo-haptic rotation, which differed between trials in the incongruent condition. The tendency to underestimate the centrifugal rotation of the hand also affected the judgment of the visual orientations. The 8 degree centripetal visual orientation was apparently still within the range of orientations that was judged as congruent, and could therefore slightly compensate for the centrifugal egocentric bias in haptic parallel matching, whereas the 16 degree centrifugal visual orientation, apparently also within the range of "congruent" orientations, strengthened the egocentric bias. The fact that we found such a hand-dependent effect of the direction of the visual deviation, indicates that visuo-haptic integration is determined by handdependent congruency. The fact that the 8 degree centrifugal information in the incongruent runs had the same effect on haptic parallel matching as the visual information in the congruent run might indicate that participants perceived this orientation as reflecting the highest level of congruence in the incongruent run.

Why did we not find an improvement with additional visual information, as reported in earlier studies (Newport et al., 2002; Zuidhoek et al., 2004)? In the current study, the way the visual information was presented did not provide any information allowing the construction of an allocentric, world-based reference frame. The visual information was only presented during the exploration, therefore only allowing to establish the transformation between one hand and the external visual reference (presenting the visual information during all task phases would have changed the task to a visual orientation matching task). This would not suffice to improve the computation of the spatial transformation of the orientation to the other hand.

It might be that the beneficial effects of non-informative vision on haptic parallel matching in previous studies (Newport et al., 2002; Zuidhoek et al., 2004) were not 
brought about by the influence of focal vision, but rather by peripheral visual input. There is evidence that different cortical systems are involved for reaching in central and peripheral vision, with a more extended cortical network including the parietooccipital junction associated with the latter (Prado et al., 2005). Peripheral vision can have a crucial influence on the perceived orientation of visually presented rods, by providing a frame of reference (Asch and Witkin, 1992). This is also demonstrated by the occurrence of the rod-and-frame illusion. In this illusion, the tilting of a peripheral visual frame induces the perception of an opposite tilt in a vertical rod (e.g. Dyde and Milner, 2002). Peripheral vision also has considerable impact on the perception and stabilization of the body in space (Berensci et al., 2005).

Our results are in accordance with the modality appropriateness hypothesis, which states that the interference by a task-irrelevant modality is reduced if processing accuracy of the task-relevant modality is high (Rock and Victor, 1964). Our findings are also in line with Ernst and Banks (2002), who showed that visuo-haptic integration occurs in a statistically optimal fashion, minimizing the variance in the final perceptual estimate. This results in a task-dependent weighting of visual and haptic information, determined by the reliability of the information obtained by vision or haptics for the task at hand. Hence, since visual size information is more reliable, size perception shows visual capture. However, changing the reliability of visual size information by adding noise increased the weight of the haptic information in determining the eventual percept.

In the mirror task, somatosensory processing accuracy is high, therefore the incongruent visual information has no effect. The congruent information, on the other hand, being task relevant, did interfere with mirror matching performance, because it biased the system to use a less task-efficient visual strategy. In the parallel task, associated with low somatosensory accuracy, performance was systematically affected by incongruent visual information. We propose that haptic tasks that require an allocentric, world-based representation might use a broader reliability range for visuo-haptic congruency, also integrating visual information which is actually incongruent.

Finally, congruent information did not improve the veridicality of parallel matching, demonstrating that vision alone is not enough to allow the computation of an allocentric reference frame. Apart from the visual orientation cues, other visual cues might be needed to help create an allocentric reference frame to infer the relative position of the body in space.

\section{Conclusion}

The current study aimed to investigate the effect of visuo-haptic (in)congruence on haptic tasks, presenting congruent and parametrically varied incongruent visual orientations during the exploration phase of a delayed haptic parallel and mirror matching task.

Our results showed that haptic mirror matching was only affected by congruent visual information, as demonstrated by a reduced accuracy. In contrast, parallel matching was affected by a range of incongruent visual orientations, parametrically modulating task 
performance, depending on the size and direction of the incongruence. Performance on the parallel task deteriorated when the visual deviation was congruent to the egocentric haptic bias, whereas it improved when the visual deviation subtly counteracted the direction of the haptic egocentric bias.

Interestingly, in contrast to previous studies, we did not find a general beneficial effect for parallel matching with vision. This indicates that peripheral vision is needed to establish the allocentric, world-based reference frame, which is required for veridical parallel matching.

We conclude that haptic task performance is not determined by a single strategy or reference frame, but flexibly adapts the weighting of visual and somatosensory information, according to task demands and the level of congruency and reliability of the information.

\section{Acknowledgement}

We thank the technical personnel of the University Maastricht for their kind assistance.

\section{References}

Asch SE, Witkin HA (1992) Studies in space orientation. II. Perception of the upright with displaced visual fields and with body tilted. J Exp Psychol Gen 121: 407-418.

Battaglia-Mayer A, Caminiti R, Lacquaniti F, Zago M (2003) Multiple levels of representation of reaching in the parieto-frontal network. Cereb Cortex 13: 1009-1022.

Berensci A, Ishihara M, Imanaka K (2005) The functional role of central and peripheral vision in the control of posture. Hum Mov Sci 24: 689-709.

Buneo CA, Jarvis MR, Batista AP, Andersen RA (2002) Direct visuomotor transformations for reaching. Nature 416: 632-636.

Burton H, Sinclair RJ (2000) Attending to and remembering tactile stimuli: A review of brain imaging data and single-neuron responses. J Clin Neurophysiol 17: 575-591.

Clower DM, Boussaoud D (2000) Selective use of perceptual recalibration versus visuomotor skill acquisition. J Neurophysiol 84: 2703-2708.

Dodds AG (1978) Hemispheric differences in tactuo-spatial processing. Neuropsychologia 16: 247-250.

Dyde RT, Milner DA (2002) Two illusions of perceived orientation: one fools all of the people some of the time; the other fools all of the people all of the time. Exp Brain Res 144: 518-527.

Ernst MO, Banks MS (2002) Humans integrate visual and haptic information in a statistically optimal fashion. Nature 415: 429-433.

Kaas AL, van Mier HI (2006). Haptic spatial matching in near peripersonal space. Exp Brain Res 170: 403413.

Kaas AL, van Mier HI, Goebel R. The neural correlates of human working memory for haptically explored object orientations. Cereb Cortex. In Press.

Kappers AML (1999) Large systematic deviations in the haptic perception of parallelity. Perception 28:1001-1012.

Kappers AML (2003) Large systematic deviations in a bimanual parallelity task: further analysis of contributing factors. Acta Psychol 114:131-145. 
Kappers AML (2004) The contributions of egocentric and allocentric reference frames in haptic spatial tasks. Acta Psychol 117:333-340.

Klatzky RL, Lederman S, Reed C (1987) There's more to touch than meets the eye: the salience of object attributes for haptics with and without vision. J Exp Psychol Gen 116: 356-369.

Lederman SJ, Klatzky RL (2004) Multisensory Texture Perception. In: Calvert G, Spence C, Stein BE (eds) The handbook of Multisensory Processes. MIT press, Cambridge MA: pp 107-122.

Minami K, Kay V, Bryden MP, Free T (1994) Laterality effects for tactile patterns. Int J Neurosci 74: 55-69. Newport R, Rabb B, Jackson SR (2002). Noninformative vision improves haptic spatial perception. Curr Biol 12:1661-1664.

Oldfield RC (1971) The assessment and analysis of handedness: The Edinburgh inventory. Neuropsychologia 9: 97-113.

Prado J, Clavagnier S, Otzenberger H, Scheiber C, Kennedy H, Perenin MT (2005) Two cortical systems for reaching in central and peripheral vision. Neuron 48: 849-858.

Rock I, Victor J (1964) Vision and touch: an experimentally induced conflict between the two senses. Science 143: 594-596.

Shimojo S, Shams L (2001) Sensory modalities are not separate modalities: plasticity and interactions. Curr Opin Neurobiol 11: 505-509.

Smith MO, Chu J, Edmonston WE Jr (1977) Cerebral lateralization of haptic perception: interaction of responses to Braille and music reveals a functional bias. Science 197: 689-690.

Valyear KF, Culham JC, Sharif N, Westwood D, Goodale MA (2006) A double dissociation between sensitivity to changes in object identity and object orientation in the ventral and dorsal visual streams: a human fMRI study. Neuropsychologia 44: 218-228.

Zangaladze A, Epstein CM, Grafton ST, Sathian K (1999) Involvement of visual cortex in tactile discrimination of orientation. Nature 401: 587-590.

Zuidhoek S, Visser A, Bredero ME, Postma A (2004) Multisensory integration mechanisms in haptic space perception. Exp Brain Res 157: 265-268. 
Chapter 4

\section{The effect of body posture on haptic spatial matching}

Based on: Kaas AL., Siep N, Van Mier HI. Body posture affects haptic egocentric but not allocentric matching. Submitted. 


\section{Abstract}

Large systematic errors occur in a haptic parallel matching task, in which participants manually explore a reference orientation and rotate a test bar to make it parallel. These errors reflect a hand-centered bias. Better results are obtained in a haptic mirror matching task, in which a test bar is turned to create the mirror image of the reference orientation in the midsagittal plane. Veridical haptic parallel matching requires an allocentric reference frame, and has been argued to prefer an extrinsic, visual reference frame. In contrast, the haptic mirror task can be performed in an egocentric frame, and evidence indicates that it favors the use of an intrinsic, body-centered frame.

In the current study, body posture was manipulated, resulting in a distortion of body-centered reference frames while head position remained the same. Participants performed haptic parallel and mirror matching either with their body forward, in a natural position, or turned sideward, the legs a 90 degree angle with the midsagittal plane of the head, which was maintained in a frontal position. In line with our expectations, we found that mirror matching accuracy was reduced in the body sideward condition, while parallel matching was unaffected. In-depth analyses revealed that this effect was most pronounced in those participants who maintained their body in a leftward position, when they used their right hand for exploration of the reference bar.

The body sideward condition forced the shoulder of the opposite arm into an uncomfortable position. Therefore, the asymmetric effects for the leftward body position in the haptic mirror task suggest that spatial representations obtained by exploration with the right hand were coded in a shoulder-centered reference frame, while the left hand used an elbow-centered representation. These results indicate that mirror matching performance, but not parallel matching performance, critically depends on body-centered representations. Furthermore, it provides preliminary evidence that different reference frames might dominate left and right hand haptic exploration, the latter using shoulder-centered representations. 


\title{
The effect of body posture on haptic spatial matching
}

\begin{abstract}
$\mathbf{M}$ aking two bars parallel requires a spatial representation in an allocentric, worldbased reference frame. When blindfolded participants turned a test bar to make it parallel to a reference bar which they manually explored, they tended to turn the test bar too far away from the midsagittal plane, i.e. in a centrifugal direction (Kappers, 1999; Newport et al., 2002; Kappers, 2003; Kaas and Van Mier, 2006; see chapter 3, figure 4). These centrifugal errors increased in size with larger distances between the hands (e.g. Kappers, 1999), indicating that hand-centered reference frames have a detrimental influence on haptic parallel matching.

In contrast, when a test bar has to be turned to make it the mirror image of a reference bar in the midsagittal plane, the resulting errors are much smaller. Mirror matching can be achieved in an allocentric reference frame, but also in an egocentric reference frame, by mirroring the position of the hands (Newport et al., 2002; Kappers, 2004; Kaas and Van Mier., 2006).

The typical response patterns for haptic parallel and mirror matching can arise from the use of a single reference frame for both tasks, intermediate between an allocentric, world-based and an egocentric hand-centered reference frame (Flanders and Soechting, 1995; Kappers, 2004). In addition, we did not find differential brain activation patterns for haptic parallel and mirror matching in an imaging study, indicating that both tasks are most likely subserved by the same neuronal substrate, including primary sensorimotor areas, parieto-occipital cortex and anterior prefrontal cortex (Kaas et al., in press; chapter 5).

Nevertheless, adding vision had a differential effect on haptic parallel and mirror matching. When participants could freely view the workspace above their hands, but not their hands, haptic mirror matching was unaffected or deteriorated (Newport et al., 2002). However, such non-informative vision improved the accuracy of haptic parallel matching in another participant sample. Zuidhoek et al. (2004) separated the effect of vision perse and orienting towards the reference hand, and found that both had a beneficial effect on haptic parallel matching accuracy.

These findings suggest that the haptic parallel task might rely more on a visual, extrinsic reference frame, while haptic mirror matching might be most efficiently solved in an intrinsic, body-centered frame.
\end{abstract}


Assuming that both tasks are indeed associated with two distinct strategies, one favoring a more visual, world-based reference frame and the other a more egocentric bodycentered reference frame, visual information should differentially affect the former, while leaving the latter unaffected. Indeed, unpublished data from our group indicate that presenting incongruent visual orientations during haptic exploration modulates parallel matching accuracy, while it does not affect mirror matching (chapter 3 of this thesis). On the other hand, changes within the egocentric reference frame, e.g. by manipulating body posture, should have the opposite differential effect.

In the current study, we investigated the effect of a manipulation of body posture on haptic matching. Participants performed a haptic parallel and a mirror matching task, either with their body forward, in a natural position, or with their body turned sideward, at a 90 degree angle with the midsagittal plane of the head. If participants use the midsagittal plane of the head as a reference in haptic orientation perception, the body posture manipulation would have no effect. However, if they use the midsagittal plane of the body, we would expect a difference between the body forward and body sideward condition. Accordingly, in the body forward condition, we expected to find small mirror matching errors, and larger centrifugal parallel matching errors. In the body sideward condition, we hypothesized no change would occur in the error size for parallel matching, while we expected mirror matching performance to be reduced. In addition, we investigated the effect of task and exploration hand, expecting higher accuracy for mirror matching than for parallel matching, and a higher accuracy for left versus right hand exploration as the left hand was found to be better at haptic spatial tasks (Dodds, 1978).

\section{Materials and Methods}

\section{Participants}

Seventeen healthy right-handed females $(23 \pm 4$ years $)$ participated in the present study, and received a gift certificate or credit points for their efforts. Only female participants were recruited for this study, to eliminate potential effects of gender differences in haptic matching performance and lateralization (e.g. Kappers, 2003; Toga and Thompson, 2003). They were all right-handed as assessed by a Dutch translation of the Edinburgh Handedness Inventory (Oldfield, 1971). Participants never saw the set-up, and received instructions and a short training before starting the experiment. The experiment was approved by the local ethics committee, and all participants gave their informed consent.

\section{Apparatus and stimuli}

The experimental set-up consisted of two turn bars $(10 \times 1 \mathrm{~cm})$ with a blunt plastic needle at each end, mounted on a table with a height of $74 \mathrm{~cm}$. The centers of the bars were $60 \mathrm{~cm}$ apart, and positioned at $11.5 \mathrm{~cm}$ from the participant's side of the table. One bar, the reference bar, was used to present a reference orientation to the participant. The other bar, the test bar, had to be turned to a matching orientation by the participant. The orientation of the bars could be read with an accuracy of 0.5 degrees. Two small round patches of cloth were attached near the edge of the table, just in 
front of the bars' centers, indicating the resting positions for the fingers. A computer program was used to present auditory cues, indicating the start and end of each trial. After finishing the experiment, subjects were asked to fill in an exit questionnaire.

\section{Procedure}

Participants were blindfolded and seated in a chair. The hands or belly never touched the edges of the table. The experimenter placed the participants' fingers on the round patches of cloth indicating the resting positions, when participants were not touching the bars. Upon hearing the first tone $(1000 \mathrm{~Hz}$ for $200 \mathrm{~ms})$, participants started the trial by simultaneously exploring the reference bar with one hand, and rotating the test bar with the other hand to turn it to a matching orientation. In the parallel condition, this was the orientation parallel to the reference orientation; in the mirror condition, it was defined as the mirror image of the reference orientation in the midsagittal plane. After six seconds, a second tone was presented (500 Hz for $200 \mathrm{~ms}$ ), indicating that the participant had to remove her hands from the bars and place them at the resting positions. In half of the trials, participants used the left hand for exploration of the reference bar on the left, and the right hand for matching with the test bar on the right, and vice versa in the other half, alternating pseudorandomly. The orientations used for the reference bar were $0,45,90$ and 135 degrees. Two different default orientations, 115 and 25 degrees, were used for the test bars.

There were two body posture conditions, in which participants either performed the trials with their body straight ahead ("body forward"; see figure 1a), or the legs aligned with the side of the table, keeping their head in a frontal position ("body sideward"; see figure $1 \mathrm{~b}$ ). Nine participants were positioned with their body to the right and eight with their body to the left. The order of the conditions was counterbalanced across participants. Both the "forward" and the "sideward" condition contained one block of parallel matching and one block of mirror matching. Per block, participants completed 2 (default orientations) x 4 (reference orientations) x 2 (left/right exploration hands) $\mathrm{x}$ 3 repetitions $=48$ trials. Participants were not given any information about their performance during the experiment. During the task, participants did not rest their arms on the table, nor touch the edges of the table. Hence, they had no reference point on the table other than the turn bars.
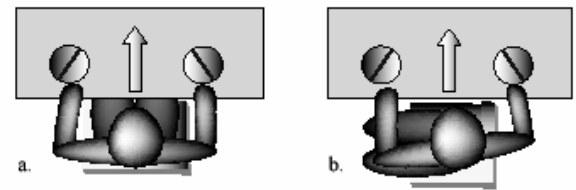

Figure 1. a: body posture in the "body forward" conditions. Participants were seated with their body midline along the center of the table. Note that the hands are not shown. b: body posture in the "body sideward" condition for the "leftward" group. Participants in the "rightward" group were seated with their body turned to the right in the "body sideward" condition. The legs were parallel to the long side of the table, at an angle of 90 degrees with respect to the table midline and the midsagittal plane of the head. The head was maintained in a frontal position. 


\section{Data analyses}

Signed errors were calculated for every trial by subtracting the reference orientation from the orientation of the test bar. For the mirror block, matching errors were expressed as deviations from the mirrored reference orientation (i.e. 45 degrees $=>135$ degrees). Errors were subsequently normalized to lie between -89 and +90 degrees and averaged within conditions. The errors in right hand exploration trials were multiplied with -1 , in order to be able to directly compare trials with left hand and right hand exploration. Hence, in the analyses, a negative error direction corresponded to the expected centrifugal error, i.e. hands turned too far away from the body's midsagittal plane, whereas a positive error corresponded to a centripetal error direction, i.e. hands turned too far towards the midsagittal plane (see see chapter 3, figure 4).

The errors were averaged over orientations, and analyzed within a four way repeated measures ANOVA with task (mirror versus parallel) x exploration hand (left/right) $\mathrm{x}$ body position (forward/sideward) as within-subject factors and side (leftward/rightward) as between-subject factor.

\section{Results}

The 2 (task) x 2 (exploration hand) x 2 (body forward/sideward) repeated measures ANOVA with side (body left/body right) as between-subjects factor revealed significant main effects for task and exploration hand, in line with our expectations. The effect of task was due to the higher average accuracy for the mirror task than the parallel task $(\mathrm{F} 1,15)=199.844 ; \mathrm{p}=0.000)$. The exploration hand effect arose from the higher average accuracy when the left hand was used for exploration and the right hand for matching than vice versa $(\mathrm{F}(1,15)=11.911 ; \mathrm{p}=0.004)$. In addition, there were three-way interactions of task by exploration hand by body side $(\mathrm{F}(1,15)=5.233 ; \mathrm{p}=0.037)$, task by exploration hand by body position $(F(1,15)=8.068 ; \mathrm{p}=0.012)$, and a four-way interaction of task by exploration hand by body position by body side $(\mathrm{F}(1,15)=4.981$; $\mathrm{p}=0.041)$. Figure 2 clearly demonstrates that these interaction effects stem from an effect in the group of participants which had their body turned to the left: they showed a much lower mirror matching accuracy when the right instead of the left hand was used for exploration of the reference bar in the body sideward condition. Importantly, the between-subject variable body side did not have a significant effect on parallel or mirror accuracy with a forward body posture (paired t-tests parallel LH exploration: $t(7)=0.246, p=0.813$; parallel $\mathrm{RH}$ exploration: $\mathrm{t}(7)=-0.431, \mathrm{p}=0.679$; mirror LH exploration: $\mathrm{t}(7)=0.004, \mathrm{p}=0.997$; mirror $\mathrm{RH}$ exploration: $\mathrm{t}(7)=-0.468, \mathrm{p}=0.654$ ).

To confirm the source of the interaction effects, we performed separate analyses for the parallel and mirror task, with exploration hand and body position as within-subject factors and body side as between-subject factor. The analysis of the parallel task showed a significant effect of exploration hand $(\mathrm{F}(1,15)=5.584 ; \mathrm{p}=0.032)$ and an interaction between exploration hand and body position $(F(1,15)=9.975 ; \mathrm{p}=0.006)$. When the left hand was used for exploration, overall accuracy was higher, and similar whether the body was in a forward position or turned sideward. When the right hand was used for exploration, there was a trend for higher accuracy in the body sideward condition (paired t-test: $\mathrm{t}(16)=-2.052, \mathrm{p}=0.057$ ). 


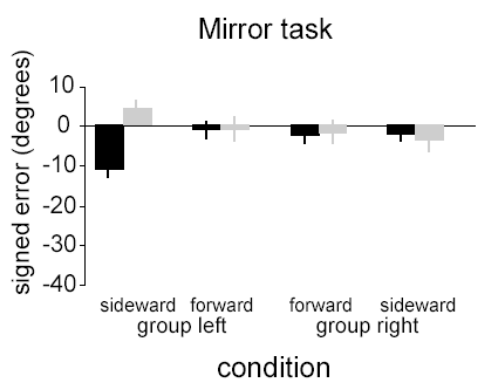

Parallel task

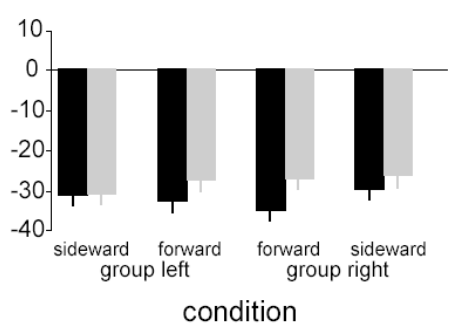

condition

right hand exploration

left hand exploration

Figure 2. Average signed matching errors and standard errors for the parallel and mirror task, displayed separately for each group and for left and right hand exploration.

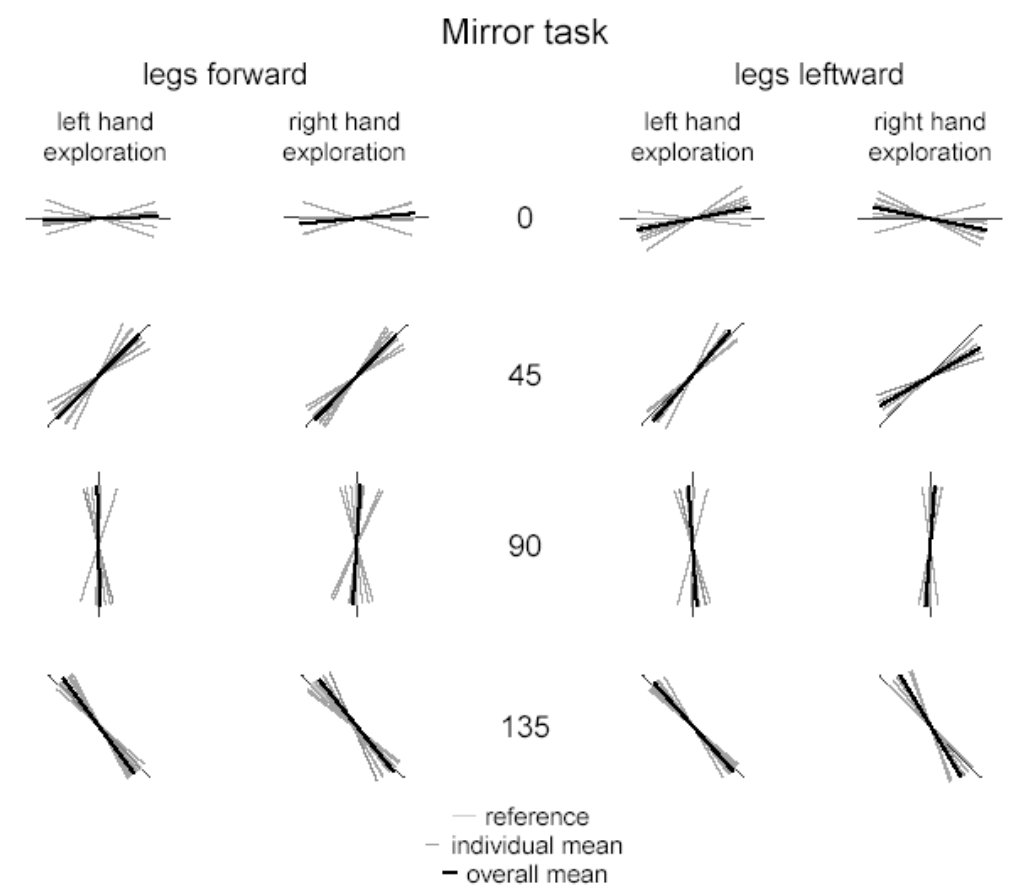

Figure 3. Group and individual average matching responses for each of the four reference orientations in the mirror task, from participants $(\mathrm{N}=8)$ executing the task with the legs forward and turned leftward. Responses were mirrored, to enable direct comparison with the reference orientation

For the mirror task (see figure 3), the exploration hand by body position by body side analysis revealed effects for body position $(\mathrm{F}(1,15)=7.742 ; \mathrm{p}=0.014)$, an exploration hand by body side interaction $(\mathrm{F}(1,15)=4.593 ; \mathrm{p}=0.049)$ and a three-way exploration hand by body position by body side interaction $(\mathrm{F}(1,15)=5.807 ; \mathrm{p}=0.029)$. Analyses for 
each exploration hand separately showed no significant effects when the left hand was used for exploration, but a significant body position effect and a body position by body side interaction $(\mathrm{F}(1,15)=6.463 ; \mathrm{p}=0.023$ and $\mathrm{F}(1,15)=7.508 ; \mathrm{p}=0.015)$ for right hand exploration. The interaction was brought about by the fact that the accuracy for right hand reference bar exploration and left hand mirror matching was only significantly reduced in the body leftward compared to the body forward condition $(\mathrm{F}(1,7)=$ 7.982; $\mathrm{p}=0.026)$. A small tendency in this direction was observed for left hand exploration and right hand mirror matching $(\mathrm{F}(1,7)=2.024 ; \mathrm{p}=0.198)$.

\section{Discussion}

Seventeen female participants performed bimanual haptic parallel and mirror matching tasks with their body either straight forward, or sideward while keeping the head oriented to the front, aligned with the center plane of the table. The resulting misalignment between the midsagittal planes of the head and body had no effect on parallel matching, while it significantly decreased accuracy for mirror matching in the midsagittal plane. Interestingly, the effect was asymmetric; it only occurred when the body was turned to the left, and was strongest when the right hand was used for exploration and the left hand for matching. Overall accuracy was higher when the left instead of the right hand was used for exploration. Finally, the current study replicated previous findings of higher accuracy for haptic parallel matching compared to mirror matching

\section{Higher accuracy for haptic mirror matching compared to parallel matching}

The small errors in mirror matching correspond to the view that haptic tasks are performed in a reference frame intermediate between an allocentric, world-based representation, and an egocentric, body-centered representation (Kappers, 2004; Flanders and Soechting, 1995). While veridical parallel matching requires an allocentric reference frame, the mirror task can be accurately solved with an egocentric strategy as well as with an allocentric strategy. Therefore, performance of the mirror task does not suffer from the egocentric bias causing the large parallel matching errors, dependent on inter manual distance. Studies regarding mirror matching using $120 \mathrm{~cm}$ distance between the bars (Kappers, 2004) found comparable deviations to those using distances of $60 \mathrm{~cm}$ or less (Newport et al., 2002; Kaas and Van Mier, 2006).

However, contrasting error directions were obtained for inter manual distances above $30 \mathrm{~cm}$ versus $30 \mathrm{~cm}$ or lower, tending to more centripetal errors in the latter (Kaas and Van Mier, 2006). Such an error direction cannot be described in the intermediate reference frame proposed by Kappers, and indicate that other mechanisms might come in to play at small inter bar distances, and at locations close to the body's midsagittal plane. In the present study, a centripetal error was observed in the body leftward condition, using the left hand for exploration. In this condition, the left hand reference bar location is close to the body midsagittal plane. No centripetal errors arose for the opposite condition, in which the body was turned rightward and exploration was performed with the right hand. Interestingly, participants in the studies by Newport et al. (2002) and Kaas and Van Mier (2006) also used the left hand for exploration. Spidalieri and Sgolastra (1999) found that left hand pointing errors to the head midline were 
strongly influenced by head-on-body position, deviating to the body midsagittal plane when the head was tilted. This might be interpreted as evidence that the representation of the head midsagittal plane and hence the mirror plane also deviated towards the left in the left hand exploration body leftward condition.

\section{Left hand superiority in haptic spatial tasks}

We found a generally higher accuracy when the left hand was used for exploration of the reference bar orientation, and the right hand for matching. This effect might be explained by the fact that haptic spatial information extracted with the left hand is first processed in the right hemisphere, which has been shown to be better suited for at spatial processing (e.g. Vallar, 1997; Toga and Thompson et al., 2003). A left hand advantage in haptic (spatial) perception has been reported in previous studies (Milner and Taylor, 1972; Kumar, 1977; Dodds, 1978; Millar and Al-Attar, 2003; Zuidhoek et al. 2005; chapter 3 of this thesis) especially in situations involving bimanual task performance (Minami et al., 1994). Zuidhoek et al. (2005) investigated the accuracy of haptic perception perse, asking participants to report the orientation of the haptically explored bar in minutes. They found left hand superiority when the verbal response was to be given immediately. However, accuracy of the right hand perception increased, equaling left hand accuracy, when a 10 second delay was imposed between haptic exploration and verbal response. This finding was interpreted to reflect a right hemispheric superiority for haptic (spatial) processing. Imaging studies have shown that the right parietal cortex is involved in the integration and working memory maintenance of tactile information for both the left and the right side of the body (Knecht et al., 1996; chapter 6 of this thesis).

\section{Different reference frames for the dominant and non-dominant hand?}

The specific effect of the body posture manipulation on haptic mirror matching indicates that this task depends on a body-centered reference frame. Conversely, more extrinsic, head-centered visual frames might be dominant in haptic parallel matching. Indeed, previous results showed that parallel matching performance was modulated by non-informative vision, gaze orientation towards the reference position (Newport et al., 2002; Zuidhoek et al. 2004) and incongruent visual orientations (chapter 3 of this thesis), whereas mirror matching was unaffected or even deteriorated by visual input. When the body was turned to one side, a considerable strain was placed on the shoulder of the opposite side, while the arm to the same side of the body could move freely. The stronger effect of a leftward versus a rightward turn therefore indicates that twisting the shoulder had a stronger effect on the right hand than on the left hand. Although the side to which the body was turned was varied between subjects in the current study, both groups performed equally well on parallel and mirror tasks with their body forward, and on the parallel task with their body sideward. Therefore, it is unlikely that the asymmetric mirror accuracy decrease in the leftward body condition be ascribed to performance differences in between the groups.

Then, how did the asymmetric effect of body position arise? Sainburg and Kalakanis (2000) compared the coordination patterns employed for the left and right arms during rapid targeted reaching movements, comparable with the movements used in our study. Their results revealed substantial differences in the coordination of left and right 
arm muscles. Unlike the left hand, the dominant right hand was able to skillfully coordinate muscle actions with inter joint dynamics. This indicates that the central nervous system developed internal models that could accurately predict musculoskeletal and environmental dynamics for the dominant hand (Bagesteiro and Sainburg, 2002). Such predictions are a prerequisite for achieving the variable control over the hand trajectory displayed by the dominant hand under different task conditions. Although the left hand could not rule out the effects of more distal muscles and joints, right hand coordination was characterized by a more proximal control strategy, in which the movement of the distal joints was primarily driven by the shoulder muscles, accurately taking into account the inter joint interactions. A contribution of the upper arm to right hand grasp orientation was also reported by Marotta et al. (2003).

In the current study, mirror matching accuracy was lowest when the body was in a leftward position, forcing the right shoulder in an uncomfortable posture. In contrast, maintaining the body in a rightward position, which forced the left shoulder into a similarly uncomfortable position, did not greatly affect mirror matching, possibly because the more distal joints were not strained by this manipulation. This suggests that the right shoulder has an important effect on both haptic exploration and matching.

When participants explored the reference bar with the right hand while maintaining a leftward body position, they experienced a strong backward pull on the right shoulder. This backward pull might be similar to what is experienced when extending and turning your right arm in a clockwise direction. Therefore, the backward pull on the right shoulder during the body leftward condition might have created the illusion that participants were feeling a more centrifugal orientation than they actually were. This, in turn, could explain why the test bar was turned to a more centrifugal orientation in this condition. Moreover, it might also explain the centripetal error direction for right hand matching.

In the body leftward condition, the error size was higher for (dominant) right hand exploration than for right hand matching (i.e.(non-dominant) left hand exploration). This implies that, in mirror matching, more serious effects are brought about by distortion of the reference frame of the hand used for exploration than for (mirror) matching. Hermens et al. (2006) found that haptic matching errors do not originate in the haptic perception or production of orientations, but rather in the transfer of the perceived bar orientation to the location of the test bar. Hence, our results might indicate an asymmetry in information transfer between the two hands.

A study by Criscimagna-Hemminger et al. (2003) showed that generalization of learned movement dynamics occurred only from the dominant to the non-dominant hemisphere, and only in extrinsic coordinates. On the other hand, Sainburg and Wang (2002) reported that there was transfer of visuomotor adaptation to the contralateral arm, independent whether the left or right arm was initially trained at reaching towards a rotated visual display. They concluded that the neural substrate controlling either arm has access to the information acquired during opposite arm learning. However, the information is used differently by each arm controller, depending on the proficiency at specifying particular movement features (i.e. final position for the left hand and initial movement direction of right hand). Since control of the left and right arm is predominantly contralateral, the observed asymmetry for body leftward and left hand exploration versus body leftward and right hand exploration might be brought about by the 
different functional specializations of the right and left hemispheres. Left hand exploration and right hand matching might optimally address the right hemisphere abilities in haptico-spatial cognition (Vallar, 1997; Knecht et al., 1996; Coghill et al., 2001; Naito et al, 2005; Zuidhoek et al., 2005; chapter 6 of this thesis) and the left hand specialization in fine motor processing and praxis (e.g. Frey et al., 2005; Gabbard et al., 2005). While the right hand might be highly proficient in accounting for inter joint interactions in motor planning, the left hand might compensate by relying more on proprioceptive feedback to adjust the end point and end posture of a movement (Sainsburg and Kalakanis, 2000). Therefore, the errors arising from the distortion of the right hand reference frame might be better compensated when the right hand is used for matching than when it is used for haptic exploration and perception.

\section{Conclusion}

The current study investigated the effect of body posture on a haptic spatial task. Participants performed haptic parallel and mirror matching either with their body forward, in a natural position, or turned sideward, at a 90 degree angle with the midsagittal plane of the head.

In line with our expectations, we found that mirror matching accuracy was reduced in the body sideward condition, while parallel matching was unaffected. In-depth analyses revealed that this effect was most pronounced in those participants who maintained their body in a leftward position, when they used their right hand for exploration of the reference bar.

These results indicate that mirror matching relies on body-centered representations, whereas parallel matching might use a more head-centered frame. The body sideward condition forced the shoulder of the opposite arm into an uncomfortable position. Therefore, the asymmetric effects for the leftward body position in the haptic mirror task, suggests that spatial representations obtained by exploration with the right hand were coded in a shoulder-centered reference frame.

\section{Acknowledgement}

We thank Johan Lataster for his help in collecting the data, and Alexander Sack for helpful comments on the manuscript.

\section{References}

Bagesteiro LB, Sainburg RL (2002) Handedness: dominant arm advantages in control oflimb dynamics. J Neurophysiol 88: 2408-2421.

Coghill RC, Gilron I, Iadarola M (2001) Hemispheric lateralization of somatosensory processing. J Neurophysiol 85: 2602-2612.

Criscimagna-Hemminger SE, Donchin O, Gazzaniga MS, Shadmehr R (2003) Learned dynamics of reaching movements generalize from dominant to non-dominant arm. J neurophysiol 89: 168-176. 
Dodds AG (1978) Hemispheric differences in tactuo-spatial processing. Neuropsychologia 16:247-250. Flanders M, Soechting JF (1995) Frames of reference for hand orientation. J Cog Neurosci 7: 182-195.

Frey SH, Funnel MG, Gerry VE, Gazzaniga MS (2005) A dissociation between the representation of tooluse skills and hand dominance: insights from left- and right-handed callosotomy patients. J Cog Neurosci 17: 262-272.

Gabbard C, Ammar D, Rodrigues L (2005) Hand effects on mentally simulated reaching. Hum Mov Sci 24: 484-495.

Hermens F, Kappers AML, Gielen SCAM (2006). The structure of fronto-parallel haptic space is task dependent. Percept Psychophys 68: 62-75.

Kaas AL, Van Mier HI (2006). Haptic spatial matching in near peripersonal space. Exp Brain Res 170: $403-$ 413.

Kaas AL, Van Mier HI, Goebel R. The neural correlates of human working memory for haptically explored object orientations. Cereb Cortex. In Press.

Kappers AML (1999) Large systematic deviations in the haptic perception of parallelity. Perception 28:1001-1012.

Kappers AML (2003) Large systematic deviations in a bimanual parallelity task: further analysis of contributing factors. Acta Psychol 114:131-145.

Kappers AML (2004) The contributions of egocentric and allocentric reference frames in haptic spatial tasks. Acta Psychol 117:333-340.

Knecht S, Kunesch E, Schnitzler A (1996) Parallel and serial processing of haptic information in man: Effects of parietal lesions on sensorimotor hand function. Neuropsychologia 34: 669-687.

Kumar S (1977) Short term memory for a nonverbal tactual task after cerebral commissurotomy. Cortex 13: 55-61.

Marotta JJ, Medendorp WP, Crawford JD (2003) Kinematic rules for upper and lower arm contributions to grasp orientation. J Neurophysiol 90: 3816-3827.

Millar S, Al-Attar Z (2003) Spatial reference and scanning movements with the left and right hand. Perception 32: 1499-1511.

Milner B, Taylor L (1972) Right-hemisphere superiority in tactile pattern-recognition after cerebral commissurotomy: evidence for nonverbal memory. Neuropsychologia 10: 1-15.

Minami K, Kay V, Bryden MP, Free T (1994) Laterality effects for tactile patterns. Int J Neurosci 74:55-69.

Naito E, Roland PE, Grefkes C, Choi HJ, Eickhoff S, Geyer S, Zilles K, Ehrsson HH (2005) Dominance of the right hemisphere and role of area 2 in human kinesthesia. J Neurophysiol 93:1020-1034.

Newport R, Rabb B, and Jackson SR (2002). Noninformative vision improves haptic spatial perception.

Curr Biol 12:1661-1664.

Oldfield RC (1971) The assessment and analysis of handedness: The Edinburgh inventory. Neuropsychologia 9: 97-113.

Sainburg RL and Kalakanis D (2000) Differences in control of limb dynamics during dominant and nondominant arm reaching. J Neurophysiol 83: 2661-2675.

Sainburg RL, Wang J (2002) Interlimb transfer of visuomotor rotations: independence of direction and final position information. Exp Brain Res 145:437-447.

Spidalieri G, Sgolastra R (1999) The head midline as a reliable reference frame for encoding head-on-body orientation. NeuroReport 10: 2473-2476.

Toga AW, Thompson PM (2003) Mapping brain asymmetry. Nat Rev Neurosci 4: 37-48. Vallar G (1997) Spatial frames of reference and somatosensory processing: a neuropsychological perspective. Philos Trans R Soc Lond B Biol Sci 352: 1401-1409. 
The effect of body posture on haptic spatial matching 79

Zuidhoek S, Visser A, Bredero ME, Postma A (2004) Multisensory integration mechanisms in haptic space perception. Exp Brain Res 157: 265-268.

Zuidhoek S, Kappers AML, Postma A (2005) Effects of hand orientation and delay on the verbal judgment of haptically perceived orientation. Perception 34: 741-755. 



\section{CHAPTER 5}

\section{The neural correlates of haptic working memory for orientation}

Based on: Kaas AL, Van Mier HI, Goebel R. The neural correlates of human working memory for haptically explored object orientations. Cereb Cortex. In Press. 


\begin{abstract}
Skillful interaction with objects requires that haptically explored spatial object characteristics such as orientation are adequately represented in working memory. In the current event-related fMRI study, seven healthy right-handed participants explored a bar-shaped reference object with the left hand and memorized its orientation. After a variable delay $(0.5,5$ or 10 seconds), participants used their right hand to match the orientation by rotating a second, identical object.

Event-related analysis of the BOLD response during the haptico-spatial working memory delay showed that right sensorimotor cortex was active in the first seconds after the end of stimulus exploration, whereas clusters in left anterior prefrontal cortex (BA 10) became dominant two seconds after the end of exploration, showing sustained activity for several seconds. In contrast, left parieto-occipital cortex was involved at the end of the delay interval.

Our results indicate that the processing of haptico-spatial information in the delay between haptic stimulus exploration and orientation matching is subserved by a dynamic network of brain areas. We propose that the haptic sensory trace, maintained in contralateral sensorimotor cortex, is transformed into a more abstract haptico-spatial representation in the early stages of the delay. The maintenance of this haptico-spatial representation engages anterior PFC and parieto-occipital cortex. While the anterior prefrontal cortex possibly integrates the spatial and motor components of haptico-spatial working memory, the parieto-occipital cortex might be involved in haptico-spatial orientation imagery, supporting working memory and the preparation of haptic matching.
\end{abstract}




\title{
The neural correlates of haptic working memory for orientation
}

\begin{abstract}
W orking memory, a concept introduced in the 1970s (Baddeley, 1998), refers to the short term maintenance and manipulation of information necessary for upcoming tasks. To date, most research on the neural substrate of (spatial) working memory has employed stimulation in the visual or auditory modality (e.g., Owen, 1997; Haxby et al., 2000; Wager and Smith, 2003). The haptic modality - or active tactile perception - has remained largely untouched. The lack of scientific attention for haptic perception might be due in part to practical problems related to stimulus presentation and response recording in the context of functional neuroimaging, as well as to the dominance of the visual modality in humans. However, haptic stimulus processing plays a key role in the skillful and sensitive interaction with our surroundings, potentially drawing upon a modality specific type of short-term memory system to keep extracted stimulus features online in the course of our daily activities.

There is some evidence that haptic working memory is characterized by three different processing stages, similar to other modalities. Most sensory details of a tactile stimulus are retained up to $500 \mathrm{~ms}$ after stimulus offset. During this stage, perceptual masking can occur, raising the intensity required for detection of a subsequent stimulus (Loomis, 1981; Craig and Rollman, 1999). The second stage is characterized by vivid recollections of uncategorized stimulus information. Although some information is lost, retention is not affected by interfering tasks until approximately 5 seconds after stimulus offset. The third stage lasts up to 30 seconds after stimulus offset, and requires rehearsal mechanisms to preserve only a limited set of feature values (Burton and Sinclair, 2000). Anatomic studies in monkeys support the idea that tactile information from the somatosensory cortex is directed ventrally through the insula to the frontal cortex for short-term storage (Burton and Sinclair, 2000; Constantinidis and Procyk, 2004). Some non-human primate studies of working memory in the somatosensory domain have shown sustained responses in primary somatosensory regions (Zhou and Fuster, 1996), parietal area 5 (Koch and Fuster, 1989) and prefrontal cortical areas (Romo et al., 1999). Recent human fMRI studies found activation in ventral prefrontal cortex for maintenance of the oblongness of a manually explored object (Stoeckel et al., 2003), and left anterior intraparietal sulcus for maintenance of kinesthetic information for subsequent haptic shape discrimination (Stoeckel et al., 2004). Dorsal prefron-
\end{abstract}


tal activation was observed when a spatiotemporal pattern of pressure pulses had to be maintained online (Numminen et al., 2004).

The present study investigated working memory maintenance of spatial representations obtained by haptic (i.e. active tactile) exploration. Haptic perception involves the integration of tactile and proprioceptive information. The existence of distinct exploration strategies to extract different types of information, like object shape and roughness, shows that it is a domain where perception and action are pre-eminently intertwined (Soechting et al., 1996). In addition, haptic spatial perception is intrinsically linked to peripersonal space, the space within a hand's reach. Psychophysical studies have shown that haptic peripersonal space is probably represented in an egocentric reference frame (centered on the body). This is indicated by systematic error patterns on tasks which appeal to allocentric reference frames (centered in the external world), like haptic parallel setting. In such a task, blindfolded participants are asked to match the orientation of a reference bar by turning a test bar to a parallel orientation. Veridical performance requires the hands to assume different postures for each bar. To achieve this, participants would need to be guided by a representation that factors out the orientation of their own body parts. However, the direction of the errors was found to be highly correlated to the natural orientation of the hand in workspace (Kappers and Koenderink, 1999; Kappers, 1999, 2004; Zuidhoek et al., 2003; Kaas and Van Mier, 2006). In contrast, a task involving haptic orientation mirroring in the mid-sagittal plane induced smaller errors. This task can be accurately performed by using a representation of the bar orientation with respect to the hands (Newport et al., 2002; Kappers, 2004; Kaas and Van Mier, 2006). There is modest empirical evidence that such egocentric representations, i.e. representations linked to the body, are associated with a different pattern of brain activity than allocentric representations, i.e. of objects with respect to other objects. When compared to allocentric judgments, egocentric judgments involving the computation of the mid-sagittal plane evoked higher bilateral parieto-frontal activation with a right-hemisphere emphasis, in fMRI studies using visual tasks (Vallar et al., 1999; Galati et al., 2000).

The current fMRI study used an allocentric and an egocentric haptic delayed match-tosample task to investigate the neural correlates of haptico-spatial working memory. In a delayed match-to-sample task, participants have to match a test stimulus to a reference stimulus which has been maintained in working memory during a delay interval. The use of delayed match-to-sample tasks in the context of event-related fMRI designs permits the segregation of neural processes related to temporally separable task components (Zarahn, 2000; Linden et al., 2003; Manoach et al., 2003). In the present study, the event-related design allowed the separation of processes related to haptico-spatial working memory from processes related to manual exploration and active manual orientation matching. Activation related to stimulus encoding (exploration), maintenance in working memory (delay) and response (haptic matching), was isolated by introducing a variable delay between the haptic exploration and matching response, with the assumption that area(s) which respond to the parametric increase of the memory delay are likely to represent the neuronal substrate for haptico-spatial working memory. The delay lengths were chosen to correspond to the hypothesized first $(0.5 \mathrm{~s})$, second (5 s) and third (10 s) haptic working memory stages (Burton and Sinclair, 2000). In addition, we explored the possibility that the distribution of activation in the cortical 
network supporting haptico-spatial working memory would be sensitive to task rules favoring either an allocentric or an egocentric representation (Vallar et al., 1999; Galati et al., 2000).

\section{Materials and methods}

\section{Participants}

Seven healthy participants ( 3 males, average age 23.7 sd 5.3 years) were paid to participate in the present study. All participants were right-handed as assessed by a Dutch translation of the Edinburgh Handedness Inventory (Oldfield, 1971). Informed consent was obtained prior to the experiment. Participants were unfamiliar with the set-up, the stimulus orientations and the purpose of the study. Six participants took part in a separate behavioral training session in the week prior to scanning, consisting of at least two runs of each task. The seventh participant practiced one run of each task on the day of the experiment. The study was approved by the local ethics committee.

\section{Experimental procedure}

Participants were blindfolded and lay supine in the scanner. Their head and arms were stabilized using foam padding. A small table was placed over the scanner bed, below the waistline, close to the bore entrance. The position of the table and the angle of the table top were adjusted to a comfortable position. Participants kept both hands on foam padding, just in front of the table. Attached to the tabletop were two turn bars $(10 \times 1 \mathrm{~cm})$, with a plastic needle at each end. The lateral motion of the arms was limited by the space within the magnet bore; therefore the bars were separated by the furthest distance that still allowed them to be comfortably reached $(35 \mathrm{~cm}$ centercenter distance).

Each trial was composed of an exploration phase (duration $1.5 \mathrm{~s}$ ), a delay phase and a matching phase (duration $2 \mathrm{~s}$ ). The delay phase could be either short $(0.5 \mathrm{~s})$, intermediate $(5 \mathrm{~s})$ or long $(10 \mathrm{~s})$. The onset of each trial was synchronized with the start of the acquisition of a new volume. The inter-trial interval (ITI) was $8.33 \mathrm{~s}$. Start and end of the exploration and matching phases were indicated by four auditory signals presented through headphones (pure tones, $1000 \mathrm{~Hz}$ for the exploration phase and $2000 \mathrm{~Hz}$ for the matching phase).

During each trial, the first auditory signal indicated that the participant had to feel the orientation of the left reference bar with the left hand. The second auditory cue signaled the end of the exploration phase and the beginning of the variable delay. Upon this cue, the participant stopped the exploration of the reference bar and positioned the left hand in the resting position on the foam padding, next to the right hand. The third auditory cue signaled the end of the delay and the beginning of the matching phase, during which the participant used the right hand to turn the test bar to an orientation matching the orientation of the reference bar (see figure 1). At the last auditory cue, indicating the end of the matching phase, the participant positioned the right hand next to the left hand in the resting position, until the start of the next trial, at which time the procedure described above was repeated (see figure 1a). 
A different matching criterion was used in each of the two experimental conditions. In the mirror task, the test bar had to be turned in such a way that its orientation matched the mirror image of the left bar with respect to the midsagittal plane of the body (see figure $1 \mathrm{~b}$ ). In the parallel task, the orientation of the test bar had to be turned parallel to the reference bar (see figure 1c).

a.

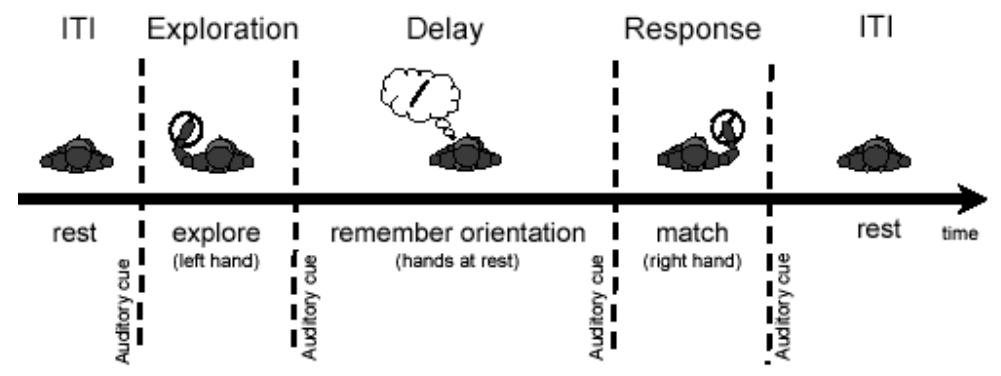

b.

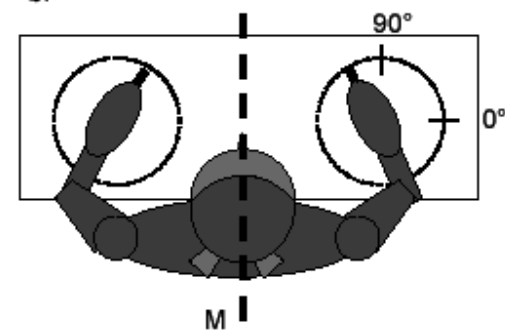

c.

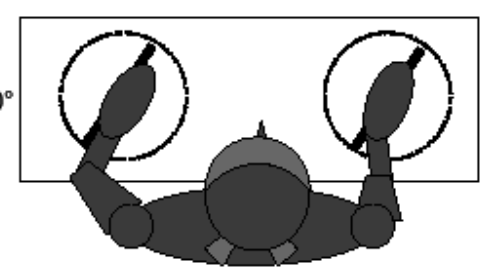

Figure 1. a: Diagram illustrating the temporal succession of task events.

Panel b and c: schematic illustration of the mirror (b) and parallel (c) matching tasks, viewed from behind the head for a participant lying in the scanner. The table top was not orthogonal with respect to the scanner bed, but slightly tilted to obtain a comfortable orientation. In the mirror task, the orientation of test bar and right hand had to be the mirror image of the orientation and hand posture on the left with respect to the midsagittal plane, which is indicated by the dashed line ' $\mathrm{M}$ ' in part b.

During all experimental runs, two assistants were present in the scanner room. For each trial, the first assistant set the reference orientations. The second assistant noted the orientation of the test bar and returned it to a default position (either $55^{\circ}$ or $125^{\circ}$, depending on the run). Accuracy for setting and recording of the orientations by the assistants was tested in advance, and was found to be within 0.5 degree. Six different orientations were used for the reference bar $\left(0^{\circ}, 30^{\circ}, 60^{\circ}, 90^{\circ}, 120^{\circ}\right.$ and $150^{\circ}$, see figure 3). Participants performed two twelve-minute runs for each task. Each run was made up of a pseudo-random series of 36 trials ( 3 delays x 6 orientations x 2 repetitions) of either the parallel or mirror task. Parallel and mirror runs were alternated, and the order was counterbalanced over participants. At the end of the experiment, participants were asked to elaborate on the strategy they had used to perform the tasks. 


\section{Behavioral data analysis}

Signed errors were calculated for every trial by subtracting the orientation of the (left) reference bar from the orientation of the (right) response bar. In the mirror task, matching errors were expressed as deviations from the inverse reference orientation (i.e. $60^{\circ}=>-60^{\circ}$ ). Errors were subsequently normalized to lie in the range $-89^{\circ}<0<$ $90^{\circ}$ and were analyzed using a 3 (delay) x 2 (task) within-subjects ANOVA.

\section{Image acquisition}

A high-resolution anatomical image was obtained from each participant in a 1.5 Tesla MR scanner using a T1-weighted MPRAGE sequence (Siemens Magnetom Sonata, matrix: 256x256x176, voxel size: $1 \times 1 \times 1 \mathrm{~mm}^{3}$ ). fMRI data were subsequently acquired in the same session using a T2*-weighted echo-planar sequence (matrix: $64 \times 64 \times 25$, voxel size: $4 \times 4 \times 4.5 \mathrm{~mm}^{3}$, $\mathrm{TE} / \mathrm{TR}=40 / 1970 \mathrm{~ms}, \mathrm{FA}=90^{\circ}$ ) covering the whole brain with the exception of the lower cerebellum. All images were obtained in one scanning session, which comprised four functional runs of on average 338 volumes. Presentation of the first auditory cue was synchronized with the fMRI sequence at the beginning of each trial.

\section{fMRI Data analysis}

The first two volumes of each run were discarded to remove T1 saturation effects. Standard preprocessing was performed, including motion correction, temporal smoothing (high pass filter, 5 cycles per run), slice scan time correction and linear trend removal, as implemented in the BrainVoyager software package 4.9/QX1.1 (Brain Innovation B.V. Maastricht, the Netherlands; Goebel et al., 2006). Functional images from different runs were aligned to the first run and coregistered with the Talairach transformed anatomical images, interpolating the functional images to obtain a volume time course with a resolution of $3 \times 3 \times 3 \mathrm{~mm}^{3}$. The resulting fMRI time series were analyzed with a general linear model (GLM). We used a design which aimed to separate different trial phases (exploration, delay, matching) and trial lengths (short, intermediate and long), using stick predictors with a width of one TR. This resulted in two predictors for the short delay trial (1 exploration, 0 delay, and 1 matching predictor), four predictors for the intermediate delay trial (1 exploration, 2 delay and 1 matching predictor), and seven for the long delay trial (1 exploration, 5 delay and 1 matching predictor), adding up to a total of thirteen predictors for each run. The predictors were shifted forward in time by two volumes (3.94 s) to account for hemodynamic sluggishness (see figure 2).

We chose stick predictors, because we did not want to make assumptions about the specific shape of the hemodynamic response, which is unlikely to be the same for all brain areas involved in the task. In addition, stick predictors allow visualization of the foci of mass activation within each TR of the delay. Previously, stick predictors were successfully applied for the detection of temporally restricted activation in the delay of a visual working memory task (Linden et al., 2003). To identify those predictors mainly reflecting the delay-related activity, we used the standard assumption that the hemodynamic response peaks approximately four seconds after the stimulus or neural event. 


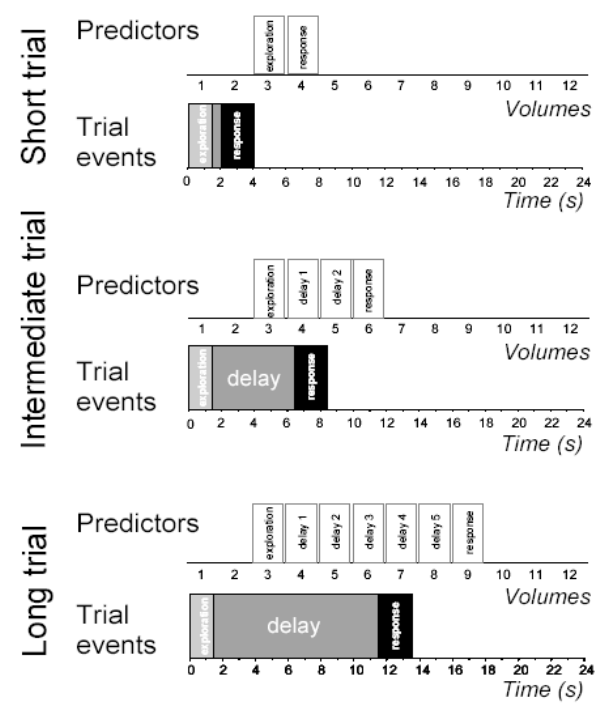

Figure 2. Trial events for each trial type (short, intermediate and long delay) and corresponding stick predictors, shifted forward in time by 2 volumes to account for the hemodynamic delay. Separate stick predictors model the exploration and response phases. The intermediate and long delays are modeled by 2 and 5 separate stick predictors, respectively.

A fixed effects group analysis was performed on 26 runs, 14 for the parallel task and 12 for the mirror task, correcting for serial correlations (one of the mirror task runs was missing in two participants, due to technical problems). In addition, single-subject analyses were performed to rule out dominance of the results by a small number of participants. Statistical maps were interpolated to a resolution of $1 \times 1 \times 1 \mathrm{~mm}^{3}$ and projected on the average of the Talairach brains of all participants. For the contrast analyses described below, only those clusters are reported which fell within gray matter and which were over $50 \mathrm{~mm}^{3}$ in size.

\section{fMRI Contrast analyses: haptic exploration and matching response}

A conjunction of the exploration and matching predictors from the short and long delay trials was used to visualize the activation in the exploration and response phases. The predictors from the short and long delay trials were used, since they were most similar (see figure 2). The matching predictor of the intermediate delay trial also included a small portion of the end of the delay, due to the uneven delay length of 5 seconds. The same conjunction of long and short trials was then also used for the exploration phase, to be able to maintain the same threshold for these very similar trial phases. In addition, the contrasts between parallel and mirror task were computed separately for exploration and matching to check for areas related to the performance of these different tasks.

\section{fMRI Contrast analyses: delay-related activity}

To disclose areas showing potential task differences, the parallel versus mirror contrast was computed for the delay phase. Subsequently, two different contrast analyses were 
used to evaluate delay-related activation. The first analysis was performed to identify brain areas showing a greater or longer response with increasing delay length, by computing the unbalanced contrast maps of all four intermediate delay trial predictors minus the two short delay trial predictors and all seven long delay trial predictors minus all four intermediate delay trial predictors. Exploration- and matching-related differences will cancel out in this analysis, since all trials were identical except for the length of the delay. In this way, regions were revealed showing a greater or longer response for increasing delay lengths. In the short delay trial, potential non-linearities in the summation of the BOLD response related to exploration and matching were assumed to be reduced or precluded by the fact that exploration and matching are carried out by different hands, with the restriction that this holds true for lateralized areas, but not for areas responding more or less independent of hand use.

The second analysis aimed to illustrate the temporal evolution of activation across the (longest) delay phase. For this purpose, a contrast map was computed for separate two-second epochs of the delay phase against ITI. Only delay epoch 2, 3 and 4 were considered, from $2 \mathrm{~s}$ after exploration offset to $2 \mathrm{~s}$ before matching onset, to reduce overlap with the descending tail of the exploration BOLD response as well as the ascending slope of the matching response. The predictor for delay epoch 2 was available from both the intermediate and long delay trials (see figure 2); for that reason a conjunction of the contrasts of this predictor against ITI from the intermediate and long delay trial was used to visualize the activation corresponding to delay epoch 2 . The predictors for delay epoch 3 and 4 were only available from the long delay trial. Areas which were stimulus related, but had a longer or shorter hemodynamic delay (time to peak) were potentially included in the delay-epoch analysis. Therefore, this analysis was used complementary to the first, and only areas that were also found in the first delay analysis, comparing trials of different delay lengths, were interpreted.

A statistical threshold of $\mathrm{q}(\mathrm{FDR})<0.05$ (false discovery rate; Genovese et al., 2002) was used for all group analyses. However, the exploration and matching phases, which entailed perceptual and motor activity, were associated with higher BOLD responses than the delay phase, which involved only cognitive processes. Therefore, the significance thresholds for figure 4 and tables 1 and 2 were set to $t(8695)>3.75$, $\mathrm{q}($ FDR $)<0.001$. Single subject analyses were thresholded at $\mathrm{p}<0.05$ (uncorrected) for all participants. In some cases $(20 \%)$, the threshold was raised to prevent the inclusion of clearly unrelated anatomical regions.

\section{Results}

\section{Behavioral performance}

The 3 (delay) by 2 (task) within-subject ANOVA on the signed matching errors did not reveal significant effects for delay lengths $(F(2,14)=0.90 ; \mathrm{p}=0.91)$, nor between mirror and parallel task $(\mathrm{F}(1,7)=1.21 ; \mathrm{p}=0.31)$. The mean signed matching error was -2.5 degree $(\mathrm{se}=4.0)$ for the parallel task, displaying a clockwise deviation, and 1.4 degree $(\mathrm{se}=2.5)$ for the mirror task, showing a counter clockwise deviation (see figure 3). 
Six out of seven participants reported having used a strategy involving some form of visualization for the parallel task. Five participants used a similar visualization strategy for the mirror task, four of whom explicitly stated that they directly recoded the reference orientations by mirroring in the 90 degree axis of the left turntable, therefore in effect performing a parallel matching with respect to this recoded reference. Two participants mentioned having used a more somatosensory strategy on the mirror task. The fact that delay did not result in a significant deterioration of performance, shows that memory traces and maintenance strategies for haptic stimuli were still effective at $10 \mathrm{sec}$ retention intervals, comparable to other psychophysical tactile studies showing retention up to at least $15 \mathrm{sec}$ (Bowers et al., 1990; Kiphart et al., 1992; Burton and Sinclair, 2000; Woods et al., 2004). Other studies reported significantly higher accuracy for mirror matching compared to parallel matching (Newport et al., 2002; Kappers, 2004; Kaas and Van Mier, 2006). However, these studies used larger distances between the turnbars in the frontoparallel plane. Decreasing the distance between the turnbars decreased the error size in haptic parallel matching (Kappers, 1999), whereas mirror matching remained unaffected (Kaas and Van Mier, 2006). The 3 (delay) by 2 (task) within-subject ANOVA on the signed matching errors did not reveal significant effects for delay lengths $(\mathrm{F}(2,14)=0.90 ; \mathrm{p}=0.91)$, nor between mirror and parallel task $(\mathrm{F}(1,7)=$ $1.21 ; \mathrm{p}=0.31)$. The mean signed matching error was -2.5 degree $(\mathrm{se}=4.0)$ for the parallel task, displaying a clockwise deviation, and 1.4 degree $(\mathrm{se}=2.5)$ for the mirror task, showing a counter clockwise deviation (see figure 3).

\section{Imaging Data}

Haptic exploration and matching response The statistical contrast between the parallel and mirror task did not yield significant results for exploration or matching. The contrast of exploration predictors against inter trial interval revealed that haptic exploration with the left hand was associated with a large activated region in the right hemisphere with its center of gravity in the central sulcus (primary motor cortex, M1). In addition, there was a large patch of activation in right lateral sulcus, including secondary somatosensory cortex and primary auditory cortex, and a smaller focus was found in right 
putamen. In the left hemisphere, activation was found in the cerebellar vermis, superior temporal cortex, left precentral sulcus, precuneus, frontal eye fields (FEF), medial occipital cortex and thalamus. Bilateral activation was observed in the ventral premotor area (vPMA) (see figure $4 \mathrm{a}$ and table 1).

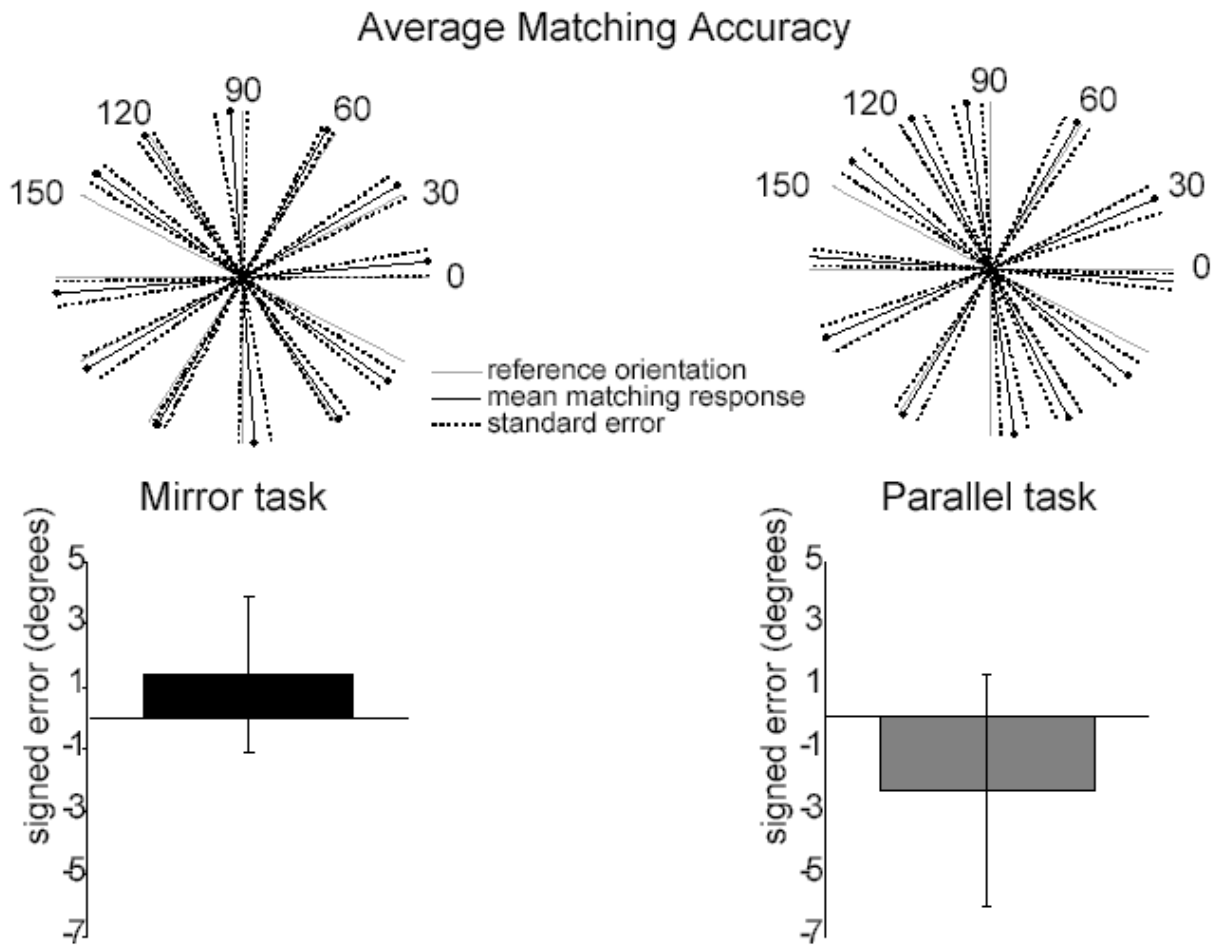

Figure 3. Average matching accuracy for mirror and parallel haptic matching. The top panel is a graphical display of the reference orientations (gray lines) and the corresponding average matching response (black lines). Dotted lines indicate the average matching response plus/minus one standard error. The panel below shows the average matching response and standard error across reference orientations.

The contrast of matching predictors against the inter-trial interval revealed the highest levels of activation in the left hemisphere. The center of gravity of the largest cluster was localized in the left primary motor cortex, consistent with use of the right hand during matching. Additional left hemispheric clusters were found in the putamen, superior temporal cortex, frontal eye fields (FEF), superior occipital cortex, superior parietal lobule, inferior temporal cortex and left precuneus. In the right hemisphere, activation clusters were centered in the insula, superior temporal gyrus, postcentral gyrus (S1) and right inferior parietal lobule. Bilateral activity was observed in cuneus and parieto-occipital sulcus (see figure $4 \mathrm{~b}$ and table 2). 


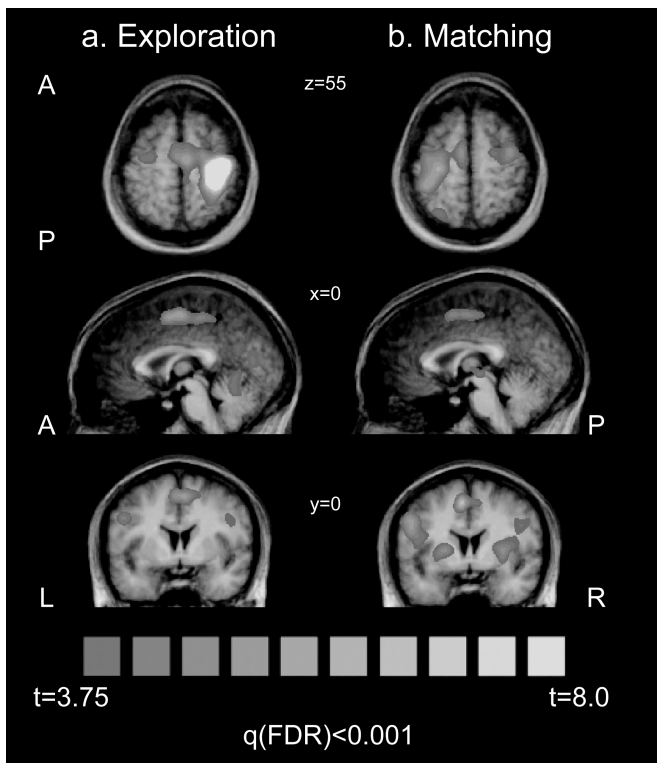

Figure 4. Statistical maps for the contrast against inter trial interval of the (a.) exploration phase and (b.) the matching phase. Maps were obtained from the fixed effects group $(\mathrm{N}=7)$ general linear model analysis, and are projected on the average of the Talairach normalized brains of all participants. The statistical threshold was set to $\mathrm{t}>3.75, \mathrm{q}(\mathrm{FDR})<0.001$. Only clusters over 50 voxels are shown, which $\mathrm{t}$-values exceeded the threshold in both short and long delay trials.

Table 1 Haptic exploration network. Areas activated for the exploration versus the inter-trial interval (ITI) contrast, conjoined for short and long trials. Voxel size is $1 \mathrm{~mm} 3$

\begin{tabular}{lrrrrrr}
\hline Brain region (COG) & BA & x & y & z & $\begin{array}{r}\text { \# of } \\
\text { voxels }\end{array}$ & $\begin{array}{r}\text { In \# } \\
\text { subjects }\end{array}$ \\
\hline L anterior cerebellum (vermis) & & & & & & \\
L superior temporal cortex & $22 / 42$ & -48 & -33 & 12 & 9317 & 7 \\
L inferior frontal sulcus (vPMA) & 44 & -44 & 3 & 29 & 1390 & 7 \\
L precentral sulcus & 6 & -27 & -10 & 53 & 1295 & 6 \\
L precuneus & 7 & -16 & -73 & 44 & 330 & 7 \\
L middle frontal gyrus (FEF) & 8 & -31 & 25 & 37 & 325 & 6 \\
L medial occipital cortex & 19 & -39 & -71 & 13 & 247 & 6 \\
L thalamus & & -7 & -20 & 1 & 64 & 7 \\
R central sulcus (M1) & 4 & 22 & -20 & 53 & 35251 & 4 \\
R superior temporal cortex & 42 & 39 & -24 & 15 & 17090 & 7 \\
R inferior frontal sulcus (vPMA) & 44 & 43 & 6 & 29 & 1966 & 7 \\
R putamen & & 28 & -6 & -1 & 78 & 7 \\
\hline
\end{tabular}

Talairach coordinates of the centers of gravity (COG) of significantly activated clusters, over 50 voxels in size, at $q(F D R)<0.001, t(8695)>3.75$. The group map was spatially smoothed with a Gaussian kernel of $8 \mathrm{~mm}$ FWHM. $L=$ left; $\mathrm{R}=$ right; $M 1=$ primary motor cortex; $S M A=$ supplementary motor area; $v P M A=$ ventral premotor cortex. 
Table 2 Haptic response network. Areas activated for the response versus ITI contrast, conjoined for short and long trials. Voxel size is $1 \mathrm{~mm} 3$

\begin{tabular}{|c|c|c|c|c|c|c|}
\hline Brain region $(\mathrm{COG})$ & $\mathrm{BA}$ & $\mathrm{x}$ & $\mathrm{y}$ & $\mathrm{z}$ & $\begin{array}{r}\text { \# of } \\
\text { voxels }\end{array}$ & $\begin{array}{r}\text { In \# } \\
\text { subjects }\end{array}$ \\
\hline L central sulcus (M1) & 4 & -12 & -16 & 52 & 26949 & 7 \\
\hline L putamen & & -27 & -3 & 13 & 15293 & 7 \\
\hline L superior temporal cortex & $22 / 42$ & -51 & -35 & 12 & 6379 & 7 \\
\hline L middle frontal gyrus (FEF) & 8 & -34 & 28 & 37 & 1733 & 6 \\
\hline L superior occipital cortex & 19 & -19 & -73 & 41 & 1288 & 6 \\
\hline L superior parietal lobule & 7 & -23 & -59 & 57 & 806 & 6 \\
\hline $\mathrm{L}$ inferior temporal cortex & 37 & -41 & -62 & 2 & 224 & 5 \\
\hline L parieto-occipital sulcus & 31 & -15 & -69 & 19 & 148 & 6 \\
\hline L precuneus & 7 & -10 & -49 & 49 & 77 & 5 \\
\hline cuneus & & 0 & -76 & 9 & 87 & 6 \\
\hline $\mathrm{R}$ insula & & 36 & 5 & 16 & 9118 & 7 \\
\hline $\mathrm{R}$ superior temporal cortex & 41 & 46 & -27 & 13 & 2655 & 7 \\
\hline $\mathrm{R}$ parieto-occipital sulcus & 31 & 14 & -64 & 14 & 1952 & 6 \\
\hline R postcentral gyrus (S1) & 2 & 33 & -32 & 48 & 963 & 7 \\
\hline $\mathrm{R}$ inferior parietal lobule & 40 & 47 & -39 & 28 & 57 & 5 \\
\hline
\end{tabular}

Delay-related activity The contrast of intermediate versus short delay trials revealed areas in the left parieto-occipital cortex (BA 19) and the ventral premotor cortex (BA 44). In the right hemisphere, primary motor cortex (M1) and occipital cortex (BA 18) were activated (see table 3a). By contrasting the long minus intermediate delay trials, significant $t$-values were found for voxel clusters in left calcarine sulcus (BA 17), left parietooccipital cortex (BA 19), left anterior prefrontal cortex (aPFC: BA 10), left putamen, bilateral middle occipital gyrus (BA 18), and right anterior cingulate gyrus (see table $3 \mathrm{~b})$. For the majority of the participants, these areas were also found to be active in single-subject analyses (see tables $3 \mathrm{a}$ and $\mathrm{b}$ ). Figure 5 displays selected activated regions and corresponding event-related time courses for both contrasts overlaid onto anatomical images, as well as the equivalent regions from the single-subject analyses. The averaged event related time course from the primary sensorimotor cluster shows a reactivation of this area in the matching phase. By contrast, left POC becomes reactivated near the end of the delay, well before the start of the matching phase, while left aPFC shows a sustained response during the first half of the long delay. The matching cue for the intermediate delay trials occured $6500 \mathrm{~ms}$ after trial onset, that is, just after volume $3(5910 \mathrm{~ms})$. The curves for left aPFC start to diverge at the next timepoint, at volume $4(7880 \mathrm{~ms})$, and the long delay trial remains high until volume 5 . 


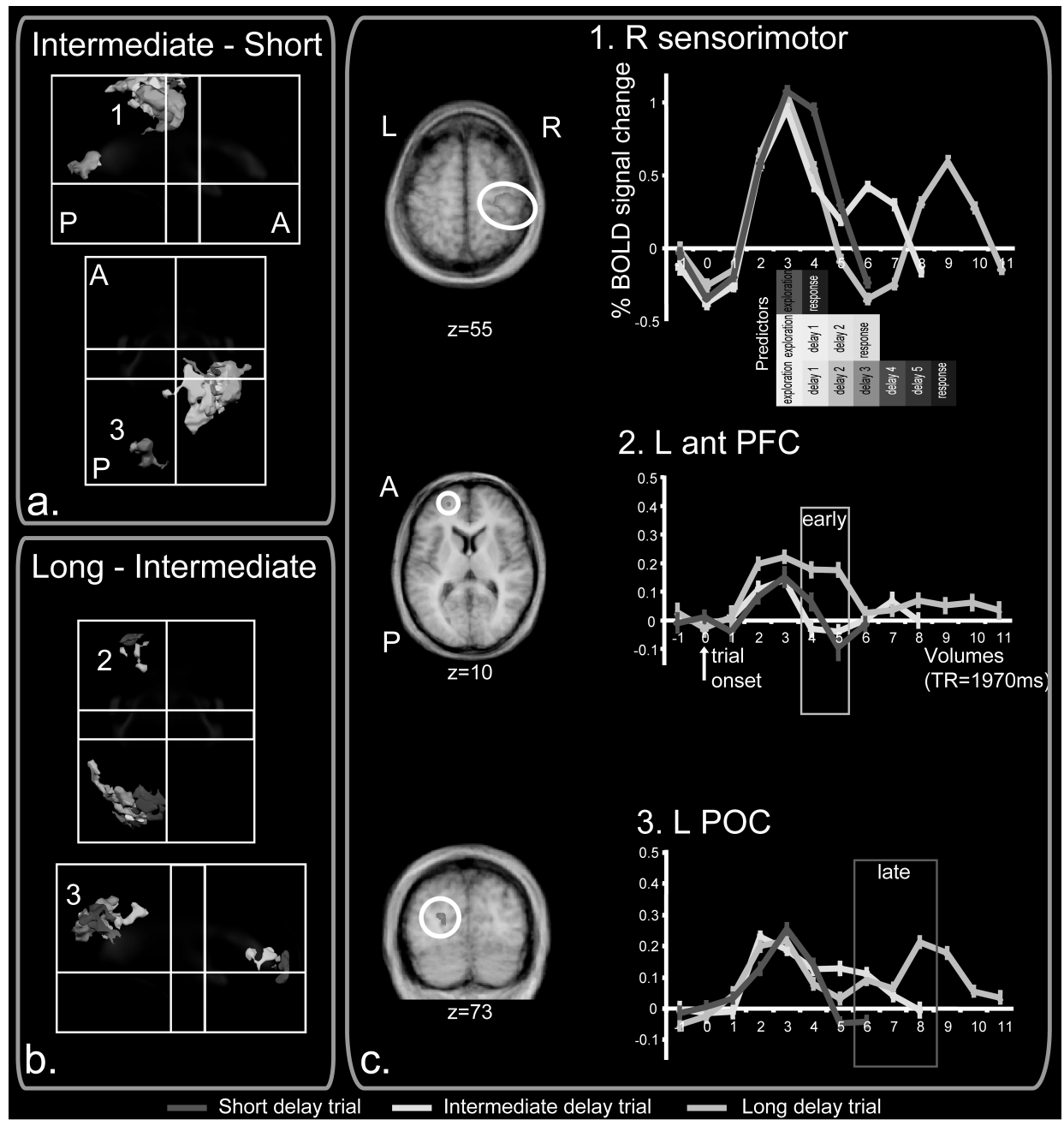

Figure 5. Selected delay-length dependent areas from single-subject (a. and b.) and group analyses (c.). Individual regions of interest for the contrast of intermediate minus short trials (a.) and long minus intermediate trials (b.) are projected in a glass brain (right and top views). $1=$ right sensorimotor cortex; $2=\mathrm{left}$ anterior prefrontal cortex ( $\mathrm{aPFC}) ; 3=$ left parieto-occipital cortex (POC). Clusters c.: The right sensorimotor cluster was obtained from the contrast of intermediate minus short delay trials (group: $t>2.9$, $\mathrm{q}(\mathrm{FDR})<0.05$; single-subject: $\mathrm{t}>2, \mathrm{p}<0.05)$, and the left aPFC cluster from the contrast of long minus intermediate trials (group: $\mathrm{t}>3.3$, $\mathrm{q}(\mathrm{FDR})<0.05$; single-subject: $\mathrm{t}>2, \mathrm{p}<0.05$ ). A similar left POC cluster was found in both contrast analyses; displayed is the time course from the cluster found by subtracting short from intermediate trials. A square window indicates the time points of interest for the left aPFC and left POC. The group clusters were projected on the group average of the Talairach normalized brains. Note that the slight spilling of the clusters from the glass brain surface is due to the fact that the surface does not reach until the superior end of Talairach space. See last pages for color version. 
The neural correlates of haptic working memory for orientation | 95

Table 3a Contrast Intermediate minus Short trial

\begin{tabular}{|c|c|c|c|c|c|}
\hline Region/Subject & $\mathrm{BA}$ & $\mathrm{x}$ & $\mathrm{y}$ & $\mathrm{z}$ & $\begin{array}{c}\text { \# of } \\
\text { voxels }\end{array}$ \\
\hline L mIFG(vPMA) & 44 & -38 & 21 & 32 & 187 \\
\hline mean $(N=5)$ & & -39 & 20 & 30 & 953 \\
\hline 2 & & -41 & 22 & 28 & 45 \\
\hline 3 & & -36 & 24 & 34 & 21 \\
\hline 5 & & -46 & 15 & 24 & 3008 \\
\hline 6 & & -38 & 18 & 28 & 1681 \\
\hline 7 & & -32 & 20 & 34 & 12 \\
\hline L SOG (POC) & 19 & -22 & -72 & 16 & 93 \\
\hline mean $(N=4)$ & & -25 & -74 & 15 & 678 \\
\hline 1 & & -29 & -74 & 14 & 188 \\
\hline 4 & & -21 & -71 & 16 & 48 \\
\hline 5 & & -27 & -73 & 17 & 506 \\
\hline 7 & & -21 & -79 & 13 & 1970 \\
\hline R CS (M1) & 4 & 32 & -26 & 58 & 3611 \\
\hline mean $(N=7)$ & & 31 & -29 & 60 & 4319 \\
\hline 1 & & 31 & -19 & 62 & 391 \\
\hline 2 & & 35 & -36 & 56 & 1166 \\
\hline 3 & & 38 & -33 & 60 & 4776 \\
\hline 4 & & 28 & -28 & 62 & 12371 \\
\hline 5 & & 27 & -33 & 63 & 3619 \\
\hline 6 & & 31 & -25 & 55 & 7750 \\
\hline 7 & & 29 & -27 & 60 & 158 \\
\hline R lingual gyrus & 18 & 12 & -86 & -9 & 156 \\
\hline mean $(N=6)$ & & 10 & -86 & -7 & 1313 \\
\hline 1 & & 28 & -90 & -4 & 900 \\
\hline 2 & & 14 & -85 & -6 & 268 \\
\hline 3 & & 15 & -87 & -9 & 102 \\
\hline 4 & & 0 & -82 & -14 & 1824 \\
\hline 5 & & 6 & -83 & -6 & 1507 \\
\hline 7 & & -2 & -90 & -5 & 3275 \\
\hline
\end{tabular}

Center-of-gravity Talairach coordinates from group analysis clusters significant at $q(\mathrm{FDR})<0.05$ and corresponding clusters from individual analyses significant at (uncorrected) $p<0.05, t>2.0$. The group map was spatially smoothed with a Gaussian kernel of $4 \mathrm{~mm}$ FWHM. Voxel size is $1 \mathrm{~mm}^{3}$. $L=$ left $; \mathrm{R}=$ right.

Group analysis $\mathrm{t}(8695)>2.9 . \mathrm{mIFG}=$ middle inferior frontal gyrus; vPMA = ventral premotor area; $S O G=$ superior occipital gyrus; $P O C=$ parieto-occipital cortex; $C S=$ central sulcus; $M 1=$ primary motor cortex.

Table 3b Contrast Long minus Intermediate trial

\begin{tabular}{cccccr}
\hline Region/Subject & BA & x & y & z & $\begin{array}{c}\text { \# of } \\
\text { voxels }\end{array}$ \\
\hline L SOG (POC) & $\mathbf{1 9}$ & $\mathbf{- 2 6}$ & $\mathbf{- 8 2}$ & $\mathbf{3 2}$ & $\mathbf{3 1 3}$ \\
mean $(\mathrm{N}=6)$ & & -30 & -79 & 33 & 1194 \\
2 & & -46 & -62 & 37 & 2089 \\
3 & -19 & -79 & 26 & 33 \\
4 & -29 & -75 & 36 & 4296 \\
5 & -25 & -84 & 39 & 284 \\
6 & -24 & -88 & 29 & 232 \\
& & -35 & -84 & 29 & 229
\end{tabular}


Table 3b Contrast Long minus Intermediate trial - continued

\begin{tabular}{|c|c|c|c|c|c|}
\hline Region/Subject & $\mathrm{BA}$ & $\mathrm{x}$ & $\mathrm{y}$ & $z$ & $\begin{array}{c}\text { \# of } \\
\text { voxels }\end{array}$ \\
\hline L putamen & & -26 & 10 & -9 & 142 \\
\hline mean $(N=7)$ & & -22 & 8 & -10 & 517 \\
\hline 1 & & -24 & 20 & -7 & 1431 \\
\hline 2 & & -27 & 7 & -15 & 379 \\
\hline 3 & & -19 & 6 & -11 & 173 \\
\hline 4 & & -28 & 4 & -11 & 288 \\
\hline 5 & & -11 & 7 & -10 & 997 \\
\hline 6 & & -23 & 9 & -7 & 38 \\
\hline 7 & & -19 & 6 & -11 & 313 \\
\hline L MOG & 18 & -11 & -95 & 7 & 104 \\
\hline mean $(N=6)$ & & -17 & -93 & 9 & 816 \\
\hline 2 & & -22 & -96 & 18 & 295 \\
\hline 3 & & -9 & -93 & -1 & 184 \\
\hline 4 & & -6 & -97 & 2 & 574 \\
\hline 5 & & -17 & -96 & 11 & 1615 \\
\hline 6 & & -35 & -80 & 10 & 1637 \\
\hline 7 & & -15 & -96 & 13 & 593 \\
\hline L CaS (V1) & 17 & -5 & -78 & 7 & 93 \\
\hline mean $(N=5)$ & & -7 & -58 & 5 & 293 \\
\hline 3 & & -5 & -84 & 0 & 849 \\
\hline 4 & & -6 & -76 & 6 & 351 \\
\hline 5 & & -5 & -76 & 8 & 195 \\
\hline 6 & & -1 & -82 & 0 & 180 \\
\hline 7 & & -3 & -76 & 8 & 22 \\
\hline L MFG (aPFC) & 10 & -21 & 49 & 10 & 57 \\
\hline mean $(N=6)$ & & -23 & 47 & 9 & 158 \\
\hline 2 & & -34 & 46 & 15 & 115 \\
\hline 3 & & -20 & 50 & 4 & 21 \\
\hline 4 & & -16 & 52 & 9 & 77 \\
\hline 5 & & -21 & 39 & 11 & 603 \\
\hline 6 & & -22 & 49 & 3 & 25 \\
\hline 7 & & -23 & 45 & 11 & 107 \\
\hline R ACG & 24 & 2 & 32 & 25 & 53 \\
\hline mean $(N=7)$ & & 5 & 16 & 18 & 518 \\
\hline 1 & & -3 & 39 & 14 & 2760 \\
\hline 2 & & 1 & 35 & 26 & 56 \\
\hline 3 & & 0 & 23 & 25 & 9 \\
\hline 4 & & 29 & -85 & 10 & 147 \\
\hline 5 & & 1 & 34 & 16 & 505 \\
\hline 6 & & 0 & 34 & 21 & 54 \\
\hline 7 & & 4 & 33 & 16 & 95 \\
\hline R MOG & 18 & 26 & -91 & 9 & 51 \\
\hline mean $(N=6)$ & & 26 & -89 & 13 & 1305 \\
\hline 2 & & 37 & -87 & 16 & 133 \\
\hline 3 & & 28 & -82 & 11 & 278 \\
\hline 4 & & 27 & -93 & 6 & 167 \\
\hline 5 & & 23 & -88 & 24 & 5530 \\
\hline 6 & & 25 & -86 & 11 & 676 \\
\hline 7 & & 16 & -98 & 9 & 1044 \\
\hline
\end{tabular}

See table 3a. group analysis: 1 (8695)>3.3. ACG = anterior cingulate gyrus; $M O G=$ medial occipital gyrus; $C a S=$ calcarine sulcus; $V 1=$ primary visual area; $a \mathrm{PFC}=$ anterior prefrontal cortex. 
The results from the epoch analysis illustrating the spatiotemporal dynamics in the delay interval are presented in figure 6 and table 4. The conjunction of the contrasts against ITI of the second delay predictor showed left hemisphere activation in the vermis of the cerebellum, aPFC (BA 10) and inferior parietal lobule (BA 40), and right hemisphere activation in the lingual and fusiform gyrus (BA 18 and 19) and the pericentral cortex (M1/S1). The contrast against ITI of the third delay predictor, available for the long delay trial only, corresponded to activation in left anterior prefrontal cortex (BA 9, BA 10) and left inferior frontal gyrus (BA 47). The contrast of the fourth delay predictor against ITI revealed significant left hemisphere clusters in the aPFC (BA 10), pre-SMA and the parieto-occipital cortex (BA 19).
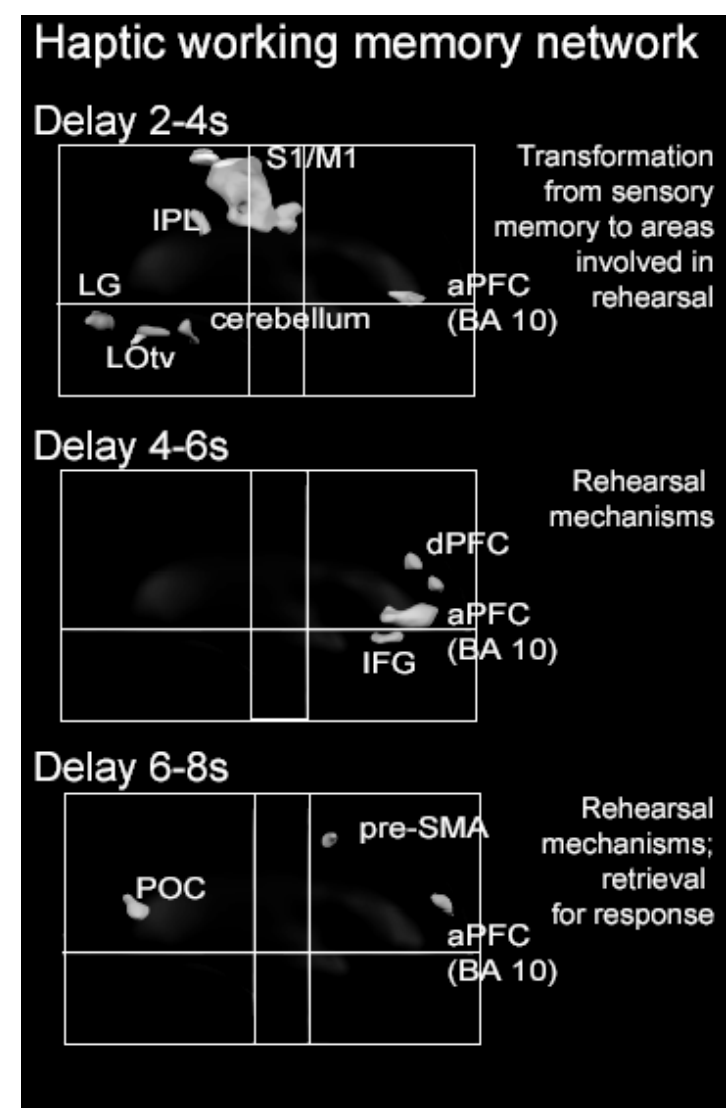

Figure 6. Surface projection of volumes of interest in a glass brain (right view), representing the dynamical neural network activated in the middle of the working memory delay. Volumes of interest for the three middle delay epochs ( 2 to $8 \mathrm{~s}$ ) were obtained from the contrasts of separate delay predictors against baseline (table 4). 
Table 4 Delay related network. Areas activated for the contrast of each of the three predictors for the middle of the delay interval against ITI.

\begin{tabular}{|c|c|c|c|c|c|c|c|}
\hline$\frac{\vec{\sigma}}{\text { อ̆ }}$ & Brain region $(\mathrm{COG})$ & $\mathrm{BA}$ & $\mathrm{x}$ & $\mathrm{y}$ & z & $\begin{array}{r}\text { \# of } \\
\text { vox- } \\
\text { els }\end{array}$ & $\begin{array}{r}\text { In \# } \\
\text { subjects }\end{array}$ \\
\hline \multirow{7}{*}{ 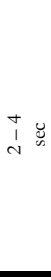 } & L middle frontal gyrus (aPFC) & $10 \mathrm{a}$ & -25 & 44 & 3 & 542 & 3 \\
\hline & L cerebellum & & -10 & -47 & -13 & 317 & 4 \\
\hline & $\mathrm{L}$ inferior parietal lobule & 40 & -28 & -43 & 38 & 316 & 4 \\
\hline & & & & & & 1076 & \\
\hline & R central sulcus (M1) & 4 & 31 & -25 & 53 & 1 & 7 \\
\hline & $\mathrm{R}$ lingual gyrus & 18 & 6 & -84 & -8 & 1251 & 4 \\
\hline & $\mathrm{R}$ fusiform gyrus (LOtv) & 19 & 48 & -64 & -14 & 408 & 6 \\
\hline \multirow{4}{*}{$\begin{array}{l}0 \\
i \\
+\end{array}$} & L middle frontal gyrus (aPFC) & $10 \mathrm{a}$ & -20 & 44 & 7 & 2198 & 7 \\
\hline & L inferior frontal gyrus & 47 & -41 & 33 & -3 & 437 & 7 \\
\hline & L superior frontal gyrus (aPFC) & $10 \mathrm{~b}$ & -13 & 54 & 21 & 371 & 6 \\
\hline & L superior frontal gyrus & 9 & -12 & 44 & 32 & 350 & 6 \\
\hline \multirow{3}{*}{$\begin{array}{l}\infty \\
1 \\
b\end{array}$} & L superior occipital gyrus (POC) & 19 & -27 & -71 & 20 & 464 & 6 \\
\hline & L superior frontal gyrus (aPFC) & $10 \mathrm{~b}$ & -13 & 56 & 23 & 334 & 6 \\
\hline & $\begin{array}{l}\text { L medial superior frontal gyrus (pre- } \\
\text { SMA) }\end{array}$ & 6 & -15 & 9 & 53 & 320 & 7 \\
\hline \multicolumn{8}{|c|}{$\begin{array}{l}\text { Center-of-gravity Talairach coordinates for significantly activated clusters over } 300 \text { voxels in size at } \\
q(\mathrm{FDR})<0.05 \text { (corresponding to } t(8695)>2.5) . \text { Group maps were spatially smoothed with a Gaussian } \\
\text { kernel of } 8 \mathrm{~mm} F W H M . \text { Voxel size is } 1 \mathrm{~mm}^{3} . \mathrm{L}=\text { left; } \mathrm{R}=\text { right; aPFC= anterior prefrontal cortex; } M 1= \\
\text { primary motor cortex; LOtv = lateral occipital tactile-visual area; } P O C=\text { parieto occipital cortex; } S M A= \\
\text { supplementary motor area. }\end{array}$} \\
\hline
\end{tabular}

\section{Discussion}

A dynamical cortical network of brain areas was revealed, using a haptic orientation matching task with a variable delay between stimulus exploration and active matching response, showing higher or sustained activation for trials of increasing delay length. Right primary sensorimotor cortex, left vPMA, right lingual gyrus (BA 18) and left parieto-occipital cortex were the main foci of activation when contrasting intermediate versus short trials, while left anterior PFC (BA 10), left parieto-occipital and bilateral medial occipital gyrus were the most important areas activated in the contrast between long and intermediate delay trials. Analysis of separate two-second delay epochs uncovered the temporal flow of mass activation in the middle of the longest delay interval, with right primary somatosensory, anterior prefrontal and parieto-occipital cortex appearing in subsequent time intervals.

\section{Activation in exploration and matching phases}

The areas revealed in the exploration and matching phases correspond to areas commonly reported to be involved in haptic sensorimotor processes (Van Mier et al., 1998, 2004; Stoeckel et al., 2003; Numminen et al., 2004; Lehéricy et al., 2005). The pattern of lateralization can be explained by the fact that the left hand was used for exploration and the right hand for matching of the stimulus bars. The activation in bilateral superior temporal regions is most likely related to the processing of the auditory stimuli, 
whereas the activations in left inferior temporal cortex in the matching phase are in line with previous reports of activation in this area during exploration of tactile objects (Pietrini et al., 2004). The involvement of occipital and parieto-occipital clusters in exploration and matching is consistent with subjective reports of visual imagery from the majority of our participants and the important role of (higher-order) visual areas for spatial processing (e.g. Zangaladze et al., 1999; Sathian and Zangaladze, 2002; Merabet et al., 2004; Zhang et al., 2004; 2005).

\section{Effect of task}

The contrast of parallel versus mirror task, separately performed for the exploration, delay and matching predictors, did not reveal any areas that responded differently during parallel or mirror task. This might indicate that the tasks are subserved by overlapping neuronal networks, which cannot be disentangled with the current spatiotemporal resolution and experimental design. The absence of a significant difference in both the behavioral measure and the imaging data might also be the result of a ceiling effect in haptic matching performance: former studies have shown that the error size for parallel matching decreases with smaller distances between the bars (Kappers, 1999; Kaas and Van Mier, 2006). However, the results could also indicate that both tasks were solved in the same, visuo-spatial reference frame. This interpretation would be in accordance with the reports of five out of seven participants stating that they had used the same strategy for both tasks.

\section{First four seconds of the delay: from sensory trace to haptico-spatial imagery}

The activity in right primary somatosensory cortex found in the contrast of intermediate and short trials and in the second (2-4 s) epoch of the delay is in line with previous findings of involvement of areas belonging to the somatosensory pathway in tactile memory (Koch and Fuster, 1989; Zhou and Fuster, 1996; Romo et al., 1999; Burton and Sinclair, 2000; Stoeckel et al., 2003; Constantinidis and Procyk, 2004; Numminen et al., 2004). The corresponding event-related time course showed substantial coactivation of the ipsilateral hemisphere in the matching phase (see figure 5). The activation of left cerebellar vermis, showing up in the second delay epoch, did not appear in the contrast of intermediate versus short delay trials. Still, the role of the right primary somatosensory area in the delay is difficult to establish based on the current fMRI study. Our interpretation would be that primary somatosensory cortex is involved in the early sensory stage of haptic working memory. This is in line with the model by Burton and Sinclair (Burton and Sinclair, 2000) and with previous studies reporting involvement of primary somatosensory cortex in short-term maintenance of vibrotactile stimuli (Harris et al., 2002) and tactile texture stimuli (Zhou and Fuster, 1996). These studies circumvented the problem of the hemodynamic confound in fMRI by using temporally resolved transcranial stimulation (TMS) and single cell recordings, respectively.

The contrast of intermediate versus short delay trials also revealed activity in left ventral premotor cortex (BA 44), which might correspond to a region involved in visuomotor transformations for grasping and manipulating objects in macaques (Rizzolatti et al., 2002) and humans (Binkofski et al., 1999; Johnson-Frey et al., 2005). Lesions of left ventral premotor cortex are observed in patients suffering from ideomotor apraxia 
(Haaland et al., 2000), a pathological condition characterized by the performance of spatiotemporal errors when pantomiming transitive tool-use gestures, as well as intransitive gestures, e.g. waving goodbye (Choi et al., 2001). The fact that this region remained when subtracting short from intermediate trials, but not when calculating long minus intermediate trials, suggests that the long and intermediate delay trials involve equal activation levels in vPMA, whereas different pathways are used for the sensorimotor transformations in the short trials.

The left parieto-occipital cluster (POC) revealed in the contrast between intermediate and short trials is similar to clusters found in the delay epoch 6-8 s after exploration offset and in the contrast between long and intermediate trials. These POC clusters might be involved in directing haptico-spatial attention (Simon et al., 2002). An area postero-medial from POC has been interpreted as the neural substrate of tactile position recognition, because of its role in integrating proprioceptive posture information on visuo-tactile matching trials performed with crossed hands (Misaki et al., 2002). Alternatively, the POC region might support specific haptico-spatial orientation imagery, supporting haptic working memory. Visual imagery has been shown to activate primary and secondary (parieto-occipital) visual areas (Amedi et al., 2005). The area found in the current study might be the human homologue of the macaque V6 complex, which contains a large representation of the periphery of the contralateral visual hemifield (Pitzalis et al., 2006). Alternatively, it might be functionally homologous to the region known as caudal intraparietal sulcus (c-IPS) in the macaque, which responded selectively to the longitudinal axis of elongated objects that were presented visually (Sakata et al., 2005). Left hemisphere areas with more dorsal coordinates than POC were reported to show preferential activation for tools as compared to other classes of objects (Johnson-Frey, 2004). In addition, parieto-occipital areas have been found to be of vital importance for tactile discrimination of macrostructural object attributes such as orientation in normally sighted humans (Sathian et al., 1997; Zangaladze et al., 1999; Sathian and Zangaladze, 2002; Zhang et al., 2005). Orientationselective adaptation effects related to visual higher-order shape processing were found in a right POC area (Talairach coordinates: 22, -81, 19; Valyear et al., 2006).

In line with the lingual gyrus activation in the present study, Stoeckel et al. found left lingual gyrus activity when a manipulandum was explored with the right hand, interpreting the activation as the neural correlate for a visual imagery strategy for the encoding of geometric properties (Stoeckel et al., 2003). Primary and secondary visual areas were also reported in a study involving mental rotation of tools (Vingerhoets et al., 2002). In our study, right medial lingual gyrus (BA 18) activity was accompanied by right LOtv (BA 19; Amedi et al., 2001) and left inferior parietal activity in the twosecond epoch approximately two to four seconds after exploration offset. This constellation of areas might support a haptico-spatial imagery strategy which aids working memory maintenance of haptic macrospatial features.

In general, the recruitment of visual areas at various stages in the current haptic orientation matching task is consistent with the dominance of vision over haptics in macrospatial tasks (Lederman and Klatzky, 2004). The functional relevance of visual areas for haptic tasks has been demonstrated by impaired haptic performance after disruption of occipital cortex by transcranial magnetic stimulation (TMS; Zangaladze et al., 1999; Merabet et al., 2004). Recruitment of visual areas can be accompanied by subjective 
experiences of visual imagery (Kosslyn et al., 2001; Ganis et al., 2004; Zhang et al., 2004). In the present study, six out of seven participants reported having used a visual imagery strategy.

\section{Four to ten seconds into the delay: the neural correlate of haptico-spatial main- tenance}

In the delay epoch from approximately 4-6 s after exploration, activation moved entirely to the prefrontal areas, with four activated clusters: one in dorsal (BA 9), one in ventral prefrontal cortex (BA 47) and two in anterior prefrontal cortex (BA 10). The last cluster was also found in the contrast of long versus intermediate delay trials, possibly representing abstract working memory maintenance of information relevant to the upcoming task (e.g. Courtney et al., 1996, 1998; D'Esposito et al., 1998; Linden et al., 2003).

Working memory theorists have long attempted to unravel the organizational principles governing the functional topography of working memory in the prefrontal cortex. One view, supported by differential anatomical connectivity and functional data, is that there is a segregation based on content domain, with working memory for objects, faces and the color or pattern of a stimulus engaging ventral portions of prefrontal cortex, and spatial working memory engaging more dorsal prefrontal areas, (Courtney et al., 1996, 1998; D'Esposito et al., 1998; Linden et al., 2003; Romanski, 2004; Mohr et al., 2006). On the other hand, evidence has been found that working memory in prefrontal cortex is organized based on the kind of cognitive processing required (Owen, 1997; Nyberg et al., 2003), with maintenance processes activating ventral areas and manipulation processes activating dorsal areas. The exact nature of the processes reflected is subject of debate. For instance, it has been argued that dorsolateral prefrontal cortex supports response selection processes rather than working memory per se (Rowe et al., 2000). In a number of studies in the visual modality, activity in PFC has been suggested to be instrumental in biasing sensory processing and response selection to ultimately achieve a desired behavioral outcome (Wallis and Miller, 2003; Reynolds and Chelazzi, 2004).

Christoff and Gabrieli proposed a rostrocaudal hierachical organization in prefrontal cortex (Christoff and Gabrieli, 2000). In their account, ventrolateral regions (BA $45 / 47 / 12$ ) are at the bottom of the hierarchy, being involved in the maintenance of one or only a few items. Dorsolateral regions (BA 9/46) have a role in the evaluation of externally generated information, and finally, frontopolar prefrontal regions (BA 10) are at the top of the hierarchy, involved in the evaluation of internally generated information. In a review integrating anatomical and functional knowledge on the anterior prefrontal (frontopolar) cortex (BA 10), Ramnani and Owen conclude that this region has a specific role in the integration of the outcomes of two or more separate cognitive operations in the pursuit of a higher behavioural goal (Ramnani and Owen, 2004). Anterior PFC has been labeled as the neural substrate for prospective memory, allowing the execution of an intended act after a delay, keeping a mental agenda of what to do when (Burgess et al., 2001). In line with these interpretations, we suggest that in the present study, aPFC might integrate the different sources of information necessary for the subsequent reproduction of the haptically explored spatial orientation, while at the same time anticipating the cue for the matching response. Anterior PFC might bring 
into register the information of areas involved in haptico-spatial imagery, e.g. (parieto)occipital cortex (BA 17/18) and the information from ventral premotor cortex (BA 44) on visuomotor transformations required for object manipulation, unleashing motor preparatory processes in areas such as supplementary motor cortex, cingulate motor cortex and premotor cortex (Van Mier et al., 1998, 2004; Toni et al., 2001, 2002; Cunnington et al., 2003) some time before the anticipated cue for the start of the matching. Although the design of the present study did not aim at drawing specific conclusions with respect to the various models of prefrontal involvement in working memory, our results do indicate that many pivotal areas in the above mentioned models are also engaged in haptic working memory. A dynamical pattern of activation was revealed in prefrontal regions across the haptic working memory delay, involving aPFC (BA10) as well as additional foci of activation were observed in dorsal and ventral prefrontal areas (BA 9 and 47). This suggests that various prefrontal regions might be involved in the haptic working memory delay, reflecting the hierarchical organization proposed by Christoff and Gabrieli (Christoff and Gabrieli, 2000). In our view, future attempts to formulate a comprehensive theoretical perspective on human working memory should no longer be based on a subset of the human sensory modalities, but should (among others) incorporate the tactile sense, which despite its pre-eminent importance for goal-directed behavior, has until recently been largely ignored.

\section{Specific implications for theories of haptic working memory}

Our study provides important insights into the neural bases of haptico-spatial working memory, identifying a network of areas supporting this crucial human function. Integrating the results obtained from the different delay analyses and comparing them to results reported in the literature, a few areas emerge which are likely to represent the neuronal correlate of haptic memory (see figure 7 ). The first region is located around the right central sulcus, the second in the left parieto-occipital cortex and the third in left anterior prefrontal cortex. The three processing stages in tactile memory proposed by Burton and Sinclair (Burton and Sinclair, 2000) might be reflected by subsequent dominance of each of these regions in the working memory delay. Consequently, the first stage of haptic sensory memory might be subserved by the right primary sensorimotor cortex for stimuli explored with the left hand, while prefrontal areas might be the most important neuronal correlate for the third stage. The second stage, characterized by vivid recollections of uncategorized stimulus information and immunity to interfering tasks, might be brought about by the temporal overlap of the descending activation in the primary somatosensory cortex and increasing dominance of occipitalparietal areas as well as prefrontal regions.

The pattern of lateralization observed in the sensorimotor and occipital areas might be largely attributable to the use of the left hand for haptic exploration and the right hand for matching. We consider it less likely that the left lateralization observed in the anterior frontal areas be influenced by a change in hand use. The involvement of right dorsolateral prefrontal cortex has been found to be independent of the hand used for tactile shape discrimination (Harada et al., 2004). Although the effect of hand use on the lateralization of haptic working memory remains to be tested, left lateralized brain activity was also revealed in the delay of the only other haptic working memory study 
known to us (Stoeckel et al., 2003). The participants in this study used their right hand for both exploration and discrimination.

Each component of the proposed memory network might provide an interesting focus for future investigation, eventually aiming to disentangle the precise contribution and functional relevance of the sub-processes involved in haptico-spatial working memory.

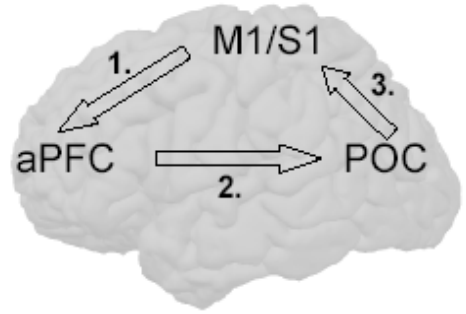

Figure 7. Schematic illustration of selected brain areas involved in haptic working memory, and potential temporal order of activation. (1) The sensory trace first stored in sensorimotor cortex (S1/M1) (2) is transformed to a more abstract haptico-spatial representation, maintained in anterior prefrontal cortex (aPFC). This representation is converted to haptico-spatial imagery by parieto-occipital cortex (POC) in the latest stages of the delay (3) preparing the haptic matching response.

\section{Conclusion}

This study represents a first attempt to unravel the dynamical cortical network involved in haptico-spatial working memory. By parametrically varying the delay length in a haptico-spatial delayed match-to-sample task, we were able to spatially map the cortical areas that showed increased activation during longer working memory delays. Our results indicate that a dynamical network of brain areas underlies working memory maintenance of haptico-spatial information in the delay between haptic stimulus exploration and orientation matching. We propose that the haptic sensory trace, maintained in contralateral sensorimotor cortex, is transformed into a more abstract hapticospatial image in the early stages of the delay. The maintenance of this haptico-spatial representation engages anterior PFC and parieto-occipital cortex. While the anterior prefrontal cortex possibly integrates the spatial and motor components of hapticospatial working memory, the parieto-occipital cortex might be involved in hapticospatial orientation imagery, supporting working memory and the preparation of haptic orientation matching.

The results of this study constitute a first step to spatiotemporally disentangle and label the subcomponents of the neural network involved in haptico-spatial working memory. Haptic working memory intricately links somatosensory, motor and cognitive processes, forming a crucial relay station between cognition and action, enabling us to shape the world around us. 


\section{Acknowledgement}

We thank Paul Gaalman and the technical personnel of the Donders center for Cognitive Neuroscience for their kind assistance, Alexander Sack, Joel Reithler and the reviewers for helpful comments.

\section{References}

Amedi A, Malach R, Hendler T, Peled S, Zohary E (2001) Visuo-haptic object-related activation in the ventral visual pathway. Nat Neurosci 4: 324-330.

Amedi A, Malach R, Pascual-Leone A (2005) Negative BOLD differentiates visual imagery and perception. Neuron 48: 859-872.

Baddeley AD. 1998. Recent developments in working memory. Curr Opin Neurobiol 8: 234-238.

Binkofski F, Buccino G, Posse S, Seitz RJ, Rizzolatti G, Freund H-J (1999) A fronto-parietal circuit for object manipulation in man: evidence from an fMRI-study. Eur J Neurosci 11: 3276-3286.

Bowers RL, Mollenhauer MS, Luxford J (1990) Short-term memory for tactile and temporal stimuli in a shared-attention recall task. Percept Mot Skills 1990 70: 903-13.

Burgess PW, Quayle A, Frith CD (2001) Brain regions involved in prospective memory as determined by positron emission tomography. Neuropsychologia 39:545-555.

Burton H, Sinclair RJ (2000) Attending to and remembering tactile stimuli: A review of brain imaging data and single-neuron responses. J Clin Neurophysiol 17: 575-591.

Choi SH, Na DL, Kang E (2001) Functional magnetic resonance imaging during pantomiming tool-use gestures. Exp Brain Res 139: 311-317.

Christoff K, Gabrieli JDE (2000) The frontopolar cortex and human cognition: Evidence for a rostrocaudal hierarchical organization within the human prefrontal cortex. Psychobiology 28: 168-186.

Constantinidis C, Procyk E (2004) The primate working memory networks. Cogn Affect Behav Neurosci 4: 444-465.

Courtney SM, Ungerleider LG, Keil K, Haxby JV (1996) Object and spatial visual working memory activate separate neural systems in human cortex. Cereb Cortex 6: 39-49.

Courtney SM, Petit L, Maisog JM, Ungerleider LG, Haxby JV (1998) An area specialized for spatial working memory in human frontal cortex. Science 279: 1347-1351.

Craig JC, Rollman GB (1999) Somesthesis. Annu Rev Psychol 50:305-331.

Cunnington R, Windischberger C, Deecke L, Moser E (2003) The preparation and readiness for voluntary movement: A high-field event-related fMRI study of the bereitschafts-bold response. Neuroimage 20: 404412.

D'Esposito M, Aguirre GK, Zarahn E, Ballard D, Shin RK, Lease J (1998) Functional MRI studies of spatial and nonspatial working memory. Brain Res Cogn Brain Res 7: 1-13.

Galati G, Lobel E, Vallar G, Berthoz A, Pizzamiglio L, Le Bihan D (2000) The neural basis of egocentric and allocentric coding of space in humans: A functional magnetic resonance study. Exp Brain Res 133: 156164.

Ganis G, Thompson WL, Kosslyn SM (2004) Brain areas underlying visual mental imagery and visual perception: An fMRI study. Brain Res Cogn Brain Res 20: 226-241.

Genovese CR, Lazar NA, Nichols T (2002) Thresholding of statistical maps in functional neuroimaging using the false discovery rate. Neuroimage 15: 870-878. 
Goebel R, Esposito F, Formisano E (2006) Analysis of FIAC data with BrainVoyager QX: From singlesubject to cortically aligned group GLM analysis and self-organizing group ICA. Hum Brain Mapp 27: 392 401.

Haaland KY, Harrington DL, Knight RT (2000) Neural representations of skilled movement. Brain 123: 2306-2313.

Harada T, Saito DN, Kashikura K, Sato T, Yonekura Y, Honda M, Sadato N (2004) Asymmetrical neural substrates of tactile discrimination in humans: a functional magnetic resonance imaging study. J Neurosci 24: 7524-7530.

Harris JA, Miniussi C, Harris IM, Diamond ME (2002) Transient storage of a tactile memory trace in primary somatosensory cortex. J Neurosci 22: 8720-8725.

Haxby JV, Petit L, Ungerleider LG, Courtney SM (2000) Distinguishing the functional roles of multiple regions in distributed neural systems for visual working memory. Neuroimage 11: 380-391.

Johnson-Frey SH (2004) The neural bases of complex tool use in humans. Trends Cogn Sci 8: 71-78.

Johnson-Frey SH, Newman-Norlund R, Grafton ST (2005) A distributed left hemisphere network active during planning of everyday tool use skills. Cereb Cortex 15: 681-695.

Kaas AL, Van Mier H (2006). Haptic spatial matching in near peripersonal space. Exp Brain Res 170: $403-$ 413.

Kappers AM, Koenderink JJ (1999) Haptic perception of spatial relations. Perception 28: 781-795.

Kappers AM (1999) Large systematic deviations in the haptic perception of parallelity. Perception 28: 1001 1012.

Kappers AML (2004) The contributions of egocentric and allocentric reference frames in haptic spatial tasks. Acta Psychol (Amst). 117:333-40.

Kiphart MJ, Hughes JL, Simmons JP, Cross HA (1992) Short-trem haptic memory for complex objects. Bull Psychon Soc 30: 212-214.

Koch C, Fuster JM (1989) Unit activity in monkey parietal cortex related to haptic perception and temporary memory. Exp Brain Res 76: 292-306.

Kosslyn SM, Ganis G, Thompson WL (2001) Neural foundations of imagery. Nature Rev Neurosci 2: 635 642.

Lederman SJ, Klatzky RL (2004) Multisensory Texture Perception. In: Calvert G, Spence C, Stein BE, editors. The handbook of multisensory processes. Cambridge, MA: The MIT Press. p 107-122.

Lehéricy S, Bardinet E, Tremblay L, Van de Moortele PF, Pochon JB, Dormont D, Kim DS, Yelnik J, Ugurbil K (2005) Motor control in basal ganglia circuits using fMRI and brain atlas approaches. Cereb Cortex 16: 149-161.

Linden DE, Bittner RA, Muckli L, Waltz JA, Kriegeskorte N, Goebel R, Singer W, Munk MH (2003) Cortical capacity constraints for visual working memory: Dissociation of fMRI load effects in a frontoparietal network. Neuroimage 20: 1518-1530.

Loomis JM (1981) Tactile pattern perception. Perception 10:5-27.

Manoach DS, Greve DN, Lindgren KA, Dale AM (2003) Identifying regional activity associated with temporally separated components of working memory using event-related functional MRI. Neuroimage 20: 1670-1684.

Merabet L, Thut G, Murray B, Andrews J, Hsiao S, Pascual-Leone A (2004) Feeling by sight or seeing by touch? Neuron 42: 173-179.

Misaki M, Matsumoto E, Miyauchi S (2002) Dorsal visual cortex activity elicited by posture change in a visuo-tactile matching task. Neuroreport 13: 1797-1800.

Mohr HM, Goebel R, Linden DEJ (2006) Content- and Task- specific dissociations of frontal activity during maintenance and manipulation in visual working memory. J Neurosci 26: 4465-4471. 
Newport R, Rabb B, Jackson SR (2002) Noninformative vision improves haptic spatial perception. Curr Biol 12: 1661-1664.

Numminen J, Schurmann M, Hiltunen J, Joensuu R, Jousmaki V, Koskinen SK, Salmelin R, Hari R (2004) Cortical activation during a spatiotemporal tactile comparison task. Neuroimage 22: 815-821.

Nyberg L, Marklund P, Persson J, Cabeza R, Forkstam C, Petersson KM, Ingvar M (2003) Common prefrontal activations during working memory, episodic memory, and semantic memory. Neuropsychologia 41: 371-377.

Oldfield RC (1971) The assessment and analysis of handedness: The edinburgh inventory. Neuropsychologia 9: 97-113.

Owen AM (1997) The functional organization of working memory processes within human lateral frontal cortex: The contribution of functional neuroimaging. European Journal of Neuroscience 9: 1329-1339.

Pietrini P, Furey ML, Ricciardi E, Gobbini MI, Wu WH, Cohen L, Guazzelli M, Haxby JV (2004) Beyond sensory images: Object-based representation in the human ventral pathway. Proc Natl Acad Sci U S A. 101:5658-5663.

Pitzalis S, Galetti C, Huang R-S, Patria F, Committeri G, Galati G, Fattori P, Sereno MI (2006) Wide-field retimotopy defines human cortical visual area V6. J Neurosci 26: 7962-7973.

Ramnani N, Owen M (2004) Anterior prefrontal cortex: insights into function from anatomy and neuroimaging. Nat Rev Neurosci 5:184-94.

Reynolds JH, Chelazzi L (2004) Attentional modulation of visual processing. Annual Review of Neuroscience 27: 611-647.

Rizzolatti G, Fogassi L, Gallese V (2002) Motor and cognitive functions of the ventral premotor cortex. Curr Opin Neurobiol 12: 149-154.

Romanski LM (2004) Domain specificity in the primate prefrontal cortex. Cogn Affect Behav Neurosci 4: 421-429.

Romo R, Brody CD, Hernandez A, Lemus L (1999) Neuronal correlates of parametric working memory in the prefrontal cortex. Nature 399: 470-473.

Rowe JB, Toni I, Josephs O, Frackowiak RS, Passingham RE (2000) The prefrontal cortex: Response selection or maintenance within working memory? Science 288: 1656-1660.

Sakata H, Tsutsui K-I, Taira M (2005) Toward an understanding of the neural processing for 3D shape perception. Neuropsychologia 43: 151-161.

Sathian K, Zangaladze A, Hoffman JM, Grafton ST (1997) Feeling with the mind's eye. Neuroreport 8: 3877-3881.

Sathian K, Zangaladze A (2002) Feeling with the mind's eye: Contribution of visual cortex to tactile perception. Behav Brain Res 135: 127-132.

Simon SR, Meunier M, Piettre L, Berardi AM, Segebarth CM, Boussaoud D (2002) Spatial attention and memory versus motor preparation: Premotor cortex involvement as revealed by fMRI. J Neurophysiol 88: 2047-2057.

Soechting JF, Tong DC, Flanders M (1996). Frames of reference in sensorimotor integration: Position sense of the arm and hand. In: Haggard P, Wing AM, Flanagan RJ, editors. Hand and brain: The neurophysiology and psychology of hand movements. San Diego, CA: Academic Press. p 151-168.

Stoeckel MC, Weder B, Binkofski F, Buccino G, Shah NJ, Seitz RJ (2003) A fronto-parietal circuit for tactile object discrimination: An event-related fMRI study. Neuroimage 19: 1103-1114.

Stoeckel MC, Weder B, Binkofski F, Choi H-J, Amunts K, Pierperhoff P, Shah NJ, Seitz RJ (2004) Left and right superior parietal lobule in tactile object discrimination. Eur J Neurosc 19: 1067-1072.

Toni I, Thoenissen D, Zilles K (2001) Movement preparation and motor intention. Neuroimage 14: S110117. 
Toni I, Thoenissen D, Zilles K, Niedeggen M (2002) Movement preparation and working memory: A behavioural dissociation. Exp Brain Res 142: 158-162.

Vallar G, Lobel E, Galati G, Berthoz A, Pizzamiglio L, Le Bihan D (1999) A fronto-parietal system for computing the egocentric spatial frame of reference in humans. Exp Brain Res 124: 281-286.

Valyear KF, Culham JC, Sharif N, Westwood D, Goodale MA (2006) A double dissociation between sensitivity to changes in object identity and object orientation in the ventral and dorsal visual streams: a human fMRI study. Neuropsychologia 44: 218-228.

Van Mier H, Tempel LW, Perlmutter JS, Raichle ME, Petersen SE (1998) Changes in brain activity during motor learning measured with PET: Effects of hand of performance and practice. J Neurophysiol 80: 2177 2199.

Van Mier HI, Perlmutter JS, Petersen SE (2004) Functional changes in brain activity during acquisition and practice of movement sequences. Motor Control 8: 500-520.

Vingerhoets G, de Lange FP, Vandemaele P, Deblaere K, Achten E (2002) Motor imagery in mental rotation: An fMRI study. Neuroimage 17: 1623-1633.

Wager TD, Smith EE (2003) Neuroimaging studies of working memory: A meta-analysis. Cogn Affect Behav Neurosci 3: 255-274.

Wallis JD, Miller EK (2003) From rule to response: Neuronal processes in the premotor and prefrontal cortex. J Neurophysiol 90: 1790-1806.

Woods AT, O'Modhrain S, Newell FN (2004) The effect of temporal delay and spatial differences on cross-modal object recognition. Cogn Affect Behav Neurosci 4: 260- 269.

Zangaladze A, Epstein CM, Grafton ST, Sathian K (1999) Involvement of visual cortex in tactile discrimination of orientation. Nature 401: 587-590.

Zarahn E (2000) Testing for neural responses during temporal components of trials with bold fMRI. Neuroimage 11: 783-796.

Zhang M, Weisser VD, Stilla R, Prather SC, Sathian K (2004) Multisensory cortical processing of object shape and its relation to mental imagery. Cogn Affect Behav Neurosci 4: 251-259.

Zhang M, Mariola E, Stilla R, Stoesz M, Mao H, Hu X, Sathian K (2005) Tactile Discrimination of Grating Orientation: fMRI Activation Patterns. Hum Brain Mapp 25: 370 -377.

Zhou YD, Fuster JM (1996) Mnemonic neuronal activity in somatosensory cortex. Proc Natl Acad Sci U S A 93: 10533-10537.

Zuidhoek S, Kappers AM, van der Lubbe RH, Postma A (2003) Delay improves performance on a haptic spatial matching task. Exp Brain Res 149: 320-330. 

CHAPTER 6

\section{The neural correlates of haptic working memory for texture}

Based on: Kaas AL, Van Mier HI, Visser M, Goebel R. The neural substrate of tactile working memory for fine surface texture revealed by parametric variation of stimulus load. Submitted. 


\section{Abstract}

A smooth object will slip from our hands when fingertip forces exceed surface friction. Hence, mental representations of tactile surface texture are crucial for adequate scaling of precision grip force. The current study investigated the neural substrate for tactile texture working memory. Ten healthy blindfolded right-handed male participants performed a delayed match-to-sample task with the dominant and non-dominant hand, in which 1 to 4 sandpaper stimuli had to be maintained in working memory during a 10 to $12 \mathrm{sec}$ delay. Functional magnetic resonance imaging revealed activation of primary and secondary somatosensory cortices during tactile encoding and matching, as well as delay-related activity in bilateral anterior inferior parietal lobule (aIPL), pre-supplementary motor area (pre-SMA) and right dorsolateral prefrontal cortex (dlPFC). Individual volume-of-interest analysis showed specific texture load dependent responses in right aIPL and right dlPFC, independent of the hand used for tactile encoding and matching. Left aIPL showed load-dependent responses and a texture preference for the dominant right hand only. Additionally, only on these dominant hand trials, right aIPL showed higher delay activation for accurate versus inaccurate trials. Pre-SMA did not reveal a higher response for the texture task compared to a location control task, nor did it show modulations for different texture loads.

Our results indicate a right-hemispheric dominance for tactile texture working memory in males. We propose that right dlPFC might subserve abstract cognitive monitoring processes and organization of multiple items in working memory. Right aIPL activation, on the other hand, might reflect domain-specific maintenance of tactile surface texture representations. 


\section{The neural correlates of haptic working memory for texture}

$\mathbf{H}_{2}$ uman grasping and skillful object manipulation require working memory maintenance of several visual and tactile object features, like orientation, shape and surface texture. Anatomic studies in monkeys showed that tactile information from the somatosensory cortex is directed ventrally through the insula to the frontal cortex for shortterm storage (Burton and Sinclair, 2000; Constantinidis and Procyk, 2004). Some nonhuman primate studies of working memory in the somatosensory domain have shown sustained responses in primary somatosensory regions (Zhou and Fuster, 1996), parietal area 5 (Koch and Fuster, 1989) and prefrontal cortical areas (Romo et al., 1999). In humans, dorsal prefrontal activation was observed when a spatiotemporal pattern of pressure pulses had to be maintained online (Numminen et al. 2004). Other recent human fMRI studies have used abstract geometric stimuli to study haptic (active tactile) working memory. For delays up to 5 seconds, maintenance of orientation information was supported by primary sensorimotor areas and parieto-occipital cortex. Maintaining higher order shape information across longer delays involved ventral prefrontal areas (Stoeckel et al., 2003; Kaas et al., in press; chapter 5), while left anterior intraparietal sulcus subserved maintenance of kinesthetic information (Stoeckel et al. 2004). In addition, these studies found delay-related activation in medial occipital, occipitoparietal and fusiform cortex. The involvement of these "visual" areas might reflect the use of a visual imagery strategy, arguing against the existence of modality-specific neural pathways for tactile working memory.

There is substantial evidence that the neural substrates for tactile processing of material features, such as roughness, are different compared to the neural substrates for tactile processing of geometrical aspects, such as orientation or distance (Klatzky et al., 1987; Randolph and Semmes, 1974; Roland et al., 1998; Bohlhalter et al., 2002; Bodegård et al., 2001; Stoesz et al., 2003; Merabet et al., 2004; Zhang et al. 2005). While common areas was observed in the primary somatosensory cortex, right hand discrimination of roughness specifically activated left secondary somatosensory (Roland et al., 1998) and right angular gyrus (Sathian et al. 1997; Zhang et al., 2005), whereas discrimination of geometrical object features recruited the left anterior intraparietal sulcus (Roland et al., 1998; Bodegård et al., 2001; Zhang et al., 2005) and left anterior supramarginal gyrus (Bodegård et al., 2001). Tactile processing of geometrical features might also involve areas common to visual processing, whereas material object features 
might be best extracted by cortical areas belonging to the tactile neural pathway. Typically, studies demonstrating a role for the visual cortex in tactile processing made use of geometrical tasks, involving objects, shapes and grating orientations in sighted participants (Sathian et al., 1997; Zangaladze et al., 1999; Amedi et al., 2001, 2002; Pietrini et al., 2004). Using grating orientations, Zangaladze et al. (1999) found that transcranial magnetic stimulation (TMS) over the visual cortex interfered with tactile discrimination. In another TMS study using Braille like stimuli, Merabet et al. (2004) found a double dissociation between micro spatial (roughness) and macro spatial (distance) processing of raised dot patterns. Low frequency rTMS over the occipital cortex of sighted and congenitally blind participants impaired tactile judgments on dot pattern spacing, while roughness judgments performed on the same tactile dot patterns remained unaffected. The reverse was true for TMS over primary somatosensory regions, which impaired judgments of roughness but not distance.

These results suggest that the likelihood that visual areas are recruited for a specific task might be modulated by the effectiveness of a visual strategy for the task at hand. In tactile geometrical tasks, the somatosensory trace might be converted to a visual image and maintained and manipulated in a temporary visual store (Kaas et al., in press; chapter 5; Bonda et al., 1996; Easton et al., 1997; Bushnell and Baxt, 1999; Deibert et al., 1999; Reales and Ballesteros, 1999). Conversely, the neural substrate specific to tactile working memory, might be revealed when tactile material properties have to be maintained online for an upcoming task.

The tactile system processes information about material properties (e.g., texture) more efficiently than information about geometrical properties (e.g., orientation; Klatzky et al., 1987). When participants were required to judge object properties which could be seen and touched, they used vision alone for geometric properties and coarse material judgments. However, to perceive subtle differences in material properties, they used touch (Klatzky et al.; 1993). Furthermore, tactile judgments of fine surface texture have been shown to be superior to visual judgments (Jones and O’Neill, 1985; Heller, 1989). While visual assessment of roughness was modulated by incongruous tactile stimulation, tactile roughness judgments were insensitive to visual interference, even when the visual distractors were more discriminable than the tactile targets (Guest and Spence, 2003). This implies that there is only partial equivalence between tactile and visual texture percepts. While the eye can only extract spatial frequency, the hand has direct access to both spatial frequency and roughness (Picard, 2006).

Therefore, to study the neural bases of tactile working memory, the current study presented blindfolded participants with sandpaper stimuli of different microgeometric texture (roughness). Subtle tactile discrimination of this common material feature is likely to primarily involve somatosensory processing, and no visual imagery or verbal strategies. Using a delayed texture match-to-sample task with varying memory load, we investigated the functional role and capacity limits of the cortical areas involved in tactile working memory. We assumed that those areas supporting tactile working memory would show activity changes for parametrical variations of stimulus load. Furthermore, we assumed a differential response for the texture task, in which tactile texture had to be maintained, and the control task, in which participants just remembered which stimulus locations were filled. Based on previous reports of activations in primary somatosensory cortex (Bodegård et al. 2001), anterior parietal cortex (Merabet 
et al., 2004), angular gyrus (Sathian et al., 1997; Zhang et al., 2005) and secondary somatosensory cortex (Roland et al., 1998) in tactile microspatial discrimination tasks, as well as activations in prefrontal cortex in tactile working memory (Klingberg et al. 1996; Numminen et al., 2004), we hypothesized that the maintenance of tactile roughness would involve these areas.

\section{Materials and methods}

\section{Participants}

Thirteen healthy male participants (average age 25 sd 3 years) were paid to participate in the present study. Data from three participants were discarded from further fMRI analyses, due to excessive head movement or average performance below chance level. The average age and standard deviation remained unchanged when computed for the remaining 10 participants. Only male participants were recruited for this study, to eliminate potential effects of gender differences that have been reported in studies of tactile performance and lateralization (e.g. Hiscock et al., 1999; Sadato et al., 2000; Toga and Thompson, 2003). All participants were right-handed as assessed by a Dutch translation of the Edinburgh Handedness Inventory (Oldfield, 1971). Informed consent was obtained prior to the experiment. The study was approved by the local ethics committee.

Participants were unfamiliar with the set-up, the stimuli and the purpose of the study. They took part in a behavioral training session prior to scanning. This session included one or more practice runs to learn the task and become proficient at correctly pacing the movements, as well as a complete behavioral experiment with the same tasks that were to be performed in the scanner.

\section{Experimental procedure}

Participants were blindfolded and lay supine in the scanner. Their head and arms were stabilized using foam padding. A small table was placed over the scanner bed below the waistline. The position of the table and the angle of the tabletop were adjusted to a comfortable position for each participant. A wooden pin on the table top marked the center of the stimulus array, and served as the default position for the index finger of the hand used for stimulus exploration. A button box was attached to the other hand. The participant's task was to perform a delayed match-to-sample task, judging whether the texture of a sample of sandpaper stimuli matched a probe, which was presented after a delay. Memory load was varied by increasing the number of stimuli in the sample from zero to four. Load zero was included as a motor control condition. Nine out of ten participants performed an additional control task, in which they judged whether the location of a sandpaper probe stimulus matched with one of the locations which was filled in the stimulus array that was previously encoded.

Each trial was composed of an encoding phase, a delay phase and a matching phase. The start of the encoding phase was indicated by an auditory warning signal $(2000 \mathrm{~Hz})$. Participants were instructed to move the index finger of the exploration hand, keeping pace with four auditory stimuli $(1000 \mathrm{~Hz}, 1 / 2 \mathrm{TR} / 985 \mathrm{~ms}$ duration), and making two lateral left-to-right sweeps along each of the four potential stimulus positions. During 
the delay phase, participants kept the index finger at the default position, on the wooden pin in the middle of the stimulus board (figure 1). The start of the matching phase was indicated by a second warning signal, alerting participants that they would have to feel the probe with two lateral left-to-right sweeps of the index finger, again paced by auditory stimuli. In the location control condition, participants also explored which was only used as a location probe. four locations in the matching phase, one of which was filled with a fixed texture

After feeling the probe, participants responded whether it was a match (left button) or a non-match (right button) by a button press with the index or middle finger of the other hand. In the load zero trials, participants responded by randomly pressing the left or right button. For the load trials, the ratio of matching and non-matching probes was set to $50 \%$ in each run. The stimulus onset asynchrony (SOA) between sample and probe was 5 or 6 TRs (TR $=1970 \mathrm{~ms} ; 5.5 \mathrm{TRs} / 10835 \mathrm{~ms}$ on average). The SOA between the probe and the next sample was a multiple of the TR, between 5 and 8 TRs (6.5 TRs/12805 ms on average). Presentation of the auditory warning signal at the beginning of each trial was synchronized with the fMRI sequence (Presentation version 9.2, Neurobehavioral Systems Inc., Albany CA, USA; nbs.neuro-bs.com).

The texture task and the location control task were performed using identical stimulus samples. The tasks were presented in two separate blocks of 10 trials (2 repetitions for each load) within one run. An auditory cue in the middle of the run indicated that participants had to switch to the other task. Both the behavioral pretest and the MRI session contained four runs, during which the experimenter presented the samples and the probes manually. All ten participants used their right hand for tactile exploration and matching. Five participants repeated the fMRI experiment with their left hand in a separate session. One participant used both left and right hand in a single session, starting with the left hand and switching to the right hand half way through the experiment. This participant only performed the texture task, completing 4 runs (40 trials) with each hand.

\section{Tactile Stimuli}

Samples of tactile stimuli were created by gluing four $1 \times 2 \mathrm{~cm}^{2}$ pieces of textured paper (sandpaper) and/or smooth paper in a square arrangement to small wooden cards $\left(10 \times 12 \mathrm{~cm}^{2}\right)$. Each wooden card contained a rectangular $1 \times 2 \mathrm{~cm}^{2}$ recess on the bottom. This allowed the card to be slid around the wooden pin which marked the default position for the participant's index finger. To ensure that participants had no trouble finding the stimulus strips, they were fixed at four standard positions with respect to the recess and by consequence also with respect to the wooden pin (figure 1).

In a separate group of participants (7 female, 8 male), a selection of six sandpaper types was tested for reliable discrimination. Each combination of two strips of different sandpaper types was presented four times. Participants explored each strip (1 x 2 $\mathrm{cm}^{2}$ ) by making two lateral sweeps with the right index finger for $985 \mathrm{~ms}(1 / 2 \mathrm{TR})$, subsequently responding whether the textures were the same or different. Results for each combination of sandpapers were analyzed across participants. Five different types of sandpaper (grain size $60,80,120,180,280$ ) showing mutual above chance discrimination, were selected to create the stimulus samples for the current fMRI experiment. 
The samples' stimulus load was varied by increasing the amount of sandpaper strips from zero to four. Strips of smooth plastic foil were used to fill the empty positions. The load zero samples consisted of four strips of smooth plastic foil, in order to provide participants with feedback that they were performing the exploration of the stimulus positions correctly. The load one samples, in turn, consisted of three pieces of smooth foil and one piece of sandpaper, load two samples of two pieces of smooth foil and two pieces of sandpaper, etc. Eight different stimulus samples were created for

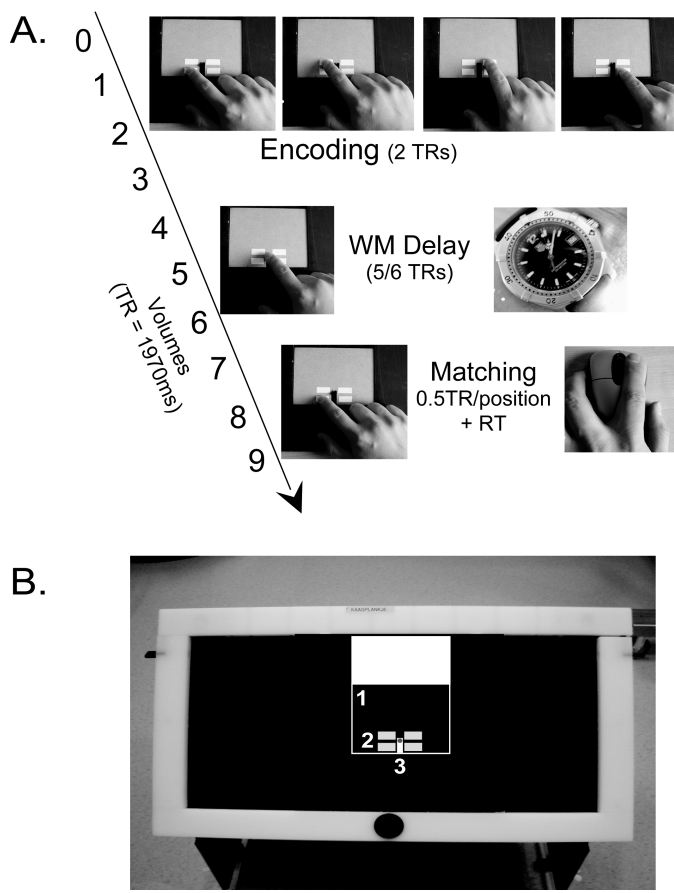

Figure 1. a: The temporal succession of the different trial phases for the texture task. While only the first probe position was explored in the matching phase of the texture task, all four positions had to be explored in the location matching task, taking 2 TRs in total. b: the table used in the scanner, with the stimulus board, and stimulus card (1) on top. For clarity, the four stimulus positions are marked by yellow squares (2) in the picture. Likewise, a red dot marks the wooden pin which served as the resting position for the finger during delay and inter trial interval. In these intervals, the stimulus cards were exchanged by carefully pulling the old card away and sliding the new card around the wooden pin, which fitted exactly in the card's recess (3).

each load, four for the pretest and four for the fMRI session. Each stimulus array was presented twice, once with a matching probe and once with a non-matching probe. To make sure performance would not be influenced by erosion of the stimulus textures, several cards were created for each stimulus array, and exchanged between participants. The stimulus samples for the texture and the location control task were identical, but the probes were different; this in order to prevent participants from matching sample and probe based on a conjunction of texture and location. In the location task, the 
probe stimulus could be in any of the four stimulus positions and always had the same texture (grain size 80). In the texture task, the probe stimulus always appeared in the same location (the first stimulus position), and was drawn from the set of five textures used to create the different sample arrays.

\section{Behavioral data analysis}

For each participant, the average percentage correct was calculated per task and load. Two participants with an average percentage correct below chance (50\% correct) in the fMRI session were excluded from further analyses. T-tests were performed to determine whether the overall average percentage correct was significantly different from chance. The effect of task and load on percentage correct was analyzed in a 2 (task) by 4 (load) within-subjects ANOVA. In addition, the effect of hand was investigated in a 2 (hand) by 4 (load) within-subjects ANOVA on the fMRI behavioral data from those participants who repeated the task with their left hand.

\section{Image acquisition}

A high-resolution anatomical image was obtained from each participant in a 3 Tesla MR scanner (Siemens Allegra, matrix: 256x256x176, voxel size: $1 \mathrm{~mm}^{3}$ ) using a T1weighted MDEFT sequence. fMRI data were subsequently acquired in the same session using a T2*-weighted echo-planar sequence (matrix: 256x256x27, voxel size: 3 $\mathrm{mm}^{3}$, gap: $1 \mathrm{~mm}, \mathrm{TE} / \mathrm{TR}=30 / 1970 \mathrm{~ms}, \mathrm{FA}=90^{\circ}$ ) covering the whole brain with the exception of the lower cerebellum. Data from nine participants were collected in a session containing four functional runs of 301 volumes. Each run included one texture and one location (control) block, composed of a pseudorandom series of 10 trials. Five participants took part in a second session, repeating the tasks with the left hand. For the tenth participant, data for left and right hand exploration were collected in a single session. To limit the time in the scanner to a reasonable amount, this participant performed the texture task only. Eight texture task runs of 150 volumes were recorded in this participant, four runs using the left hand and four runs using the right hand, resulting in the same amount of texture task data for each hand as for the other participants.

\section{fMRI Data analysis}

The first two volumes of each run were discarded to remove T1 saturation effects. Standard preprocessing was performed, including slice scan time correction, motion correction, temporal smoothing (high pass filter, 3 cycles per run) and linear trend removal, as implemented in the BrainVoyager software package QX version 1.6 (Goebel et al., 2006; Brain Innovation B.V. Maastricht, the Netherlands; www.brainvoyager.com). Functional images from different runs were coregistered with the Talairach transformed anatomical images, interpolating the functional images to obtain a volume time course with a resolution of $3 \times 3 \times 3 \mathrm{~mm}^{3}$. One participant with motion correction parameters exceeding $2 \mathrm{~mm}$ was excluded from further analyses.

A fixed-effects multiple-regression analysis was performed to reveal the areas involved in tactile texture encoding, delay and matching. The design matrix included 18 predictors for each task and hand. Fifteen predictors modeled the three trial phases (encoding/delay/matching) for the five stimulus loads (0-4). Three confound predictors were added to account for the encoding, delay and matching phase of incorrectly presented trials (one for location and one for texture, in two different participants). Each predic- 
tor's box car function was convolved with a gamma distribution, accounting for the shape and delay of the hemodynamic response (Boynton et al., 1996). Separate contrasts against baseline (i.e. the inter trial interval) were computed for texture encoding, delay and matching, across loads $(1+2+3+4)$ and conjoined for left and right hand. In addition, a simple task contrast (texture minus location) was computed separately for encoding and delay, across hands and loads $(1+2+3+4)$.

Texture delay Volumes of Interest (VOIs) were defined, by running a multiple regression analysis on a single run for each hand from each individual participant, using the contrast of texture delay predictors (load $1+2+3+4$ ), conjoined for left and right hand. Only clusters which were found in all participants were included in the subsequent analysis, in which the remaining runs from each participant were used to test the following texture delay contrasts for left and right hand separately: load (load 1-4 versus load 0; later referred to as "simple load contrast"), task (texture 1-4 versus location 1-4) and accuracy (delay of trials resulting in accurate minus the delay preceding inaccurate responses). The simple load contrast and the task contrast included only delay predictors from trials resulting in an accurate response. The respective design matrix contained 18 predictors per task: five encoding, five matching, one delay load 0, one for delay loads 1 to 4 , and three covering the three trial phases for trials resulting in an inaccurate response. The encoding, delay and matching phase of the two incorrectly presented trials were modeled by three separate confound predictors. To test for modulatory effects of load in the individual VOIs, a second design matrix was used, which was almost identical to the first, the only difference being that it contained separate predictors for each delay load. This allowed multiplication of the texture delay load 1 to 4 predictor betas for the accurate trials of each hand with the parametric load contrast $(-3-1+1+3)$. The alpha level for the one-sided t-tests was set to $p=0.05$, and $\mathrm{p}=0.00625$ after Bonferroni correction for multiple testing.

\section{Results}

\section{Task performance}

Two participants were excluded from the analyses because their average percentage correct for the texture task in the scanner was below chance level (50\%). One additional participant was left out, because his head movement exceeded the $2 \mathrm{~mm}$ threshold. For the remaining participants, the average percentage correct significantly differed from chance for all combinations of load and task. The 2 (task) by 4 (load) within-subject ANOVA $(\mathrm{N}=9)$ on the percentage correct revealed a significant main effect for task $(\mathrm{F}(1,8)=9.95 ; \mathrm{p}=0.014)$ with better performance in the location control task, and an interaction between task and load $(\mathrm{F}(3,24)=3.01 ; \mathrm{p}=0.05)$. As can be seen in figure 2 , the interaction was caused by a differential response for texture and location task to the change from load 2 to 3 (within-subjects contrast: $F(1,8)=14.18$; $\mathrm{p}=0.006$ ). The 4 (load) by 2 (hand) within-subjects ANOVA on the fMRI texture task data from the subgroup of six participants who performed the texture task with both hands, revealed a significant load effect $(\mathrm{F}(3,15)=4.05 ; \mathrm{p}<0.05)$, but no significant effect of hand, nor a load by hand interaction. 


\section{Imaging Data}

Overall analysis of Texture encoding, delay and matching The conjunction of the left and right hand encoding contrast (across load 1 to 4) against the inter trial interval, thresholded at $\mathrm{t}(17521)=14(\mathrm{p}($ Bonf $)<0.0005)$, revealed activation in bilateral primary and secondary somatosensory cortices, bilateral insular cortices, medial supplementary motor area (SMA) and right ventral premotor cortex (table 1 and figure 3).

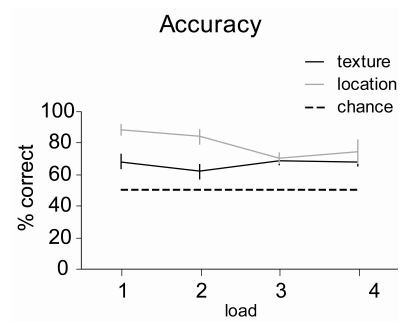

Figure 2. Average percentage correct and (between-subjects) standard error bars for each task and load, as obtained from the nine participants who performed both texture and location task, and who were included in the fMRI analyses.

The statistical map for texture delay load 1 to 4 showed activation for task performance with both left and right hand exceeding $\mathrm{t}(17521)=7(\mathrm{p}($ Bonf $)<0.0005)$ in bilateral inferior parietal lobule (IPL), medial pre-SMA and right dorsolateral prefrontal cortex (dlPFC; table 2 and figure 3). Volumes-of-Interest could be defined for all ten participants in these four regions (table 2 and figure 4; different colors represent different participants in figure 4a). Event-related averages are shown for the clusters obtained from the group analysis.

Texture matching, i.e. feeling the probe with either left or right hand and pressing the response button with the other hand, activated areas in bilateral primary and secondary somatosensory cortices, bilateral thalamus and left putamen, bilateral insular cortices, medial (pre-)SMA and right ventral premotor cortex (vPMA), with t-values at $t(17521)=7$ or higher $(\mathrm{p}($ Bonf $)<0.0005$; table 3 and figure 3$)$. The contrast of texture encoding minus location encoding, computed across hands and loads, revealed a single cluster in right secondary somatosensory cortex at $\mathrm{t}=7$ ( $\mathrm{p}(\mathrm{B}$ onf $)<0.0005$; Talairach coordinates: $60,-15,14)$. No significant differences were revealed for texture versus location in the delay phase. It was not computed for the matching phase, since this phase differed in length in the texture and location task.

Individual texture delay volume-of-interest analysis Multisubject texture delay VOI analyses revealed differential responses in right and left aIPL (figure 5), right dlPFC and preSMA (figure 6). Right aIPL showed Bonferroni-corrected significance for the simple load contrast for both hands, as well as for the accuracy and task contrast for the right hand and the parametric load contrast for left hand. Without correction, the parametric load was also significant for the right hand. Left aIPL only showed a significant effect 
for right hand performance, with Bonferroni-corrected significance for the simple load contrast, and uncorrected significance for the task and parametric load contrasts.

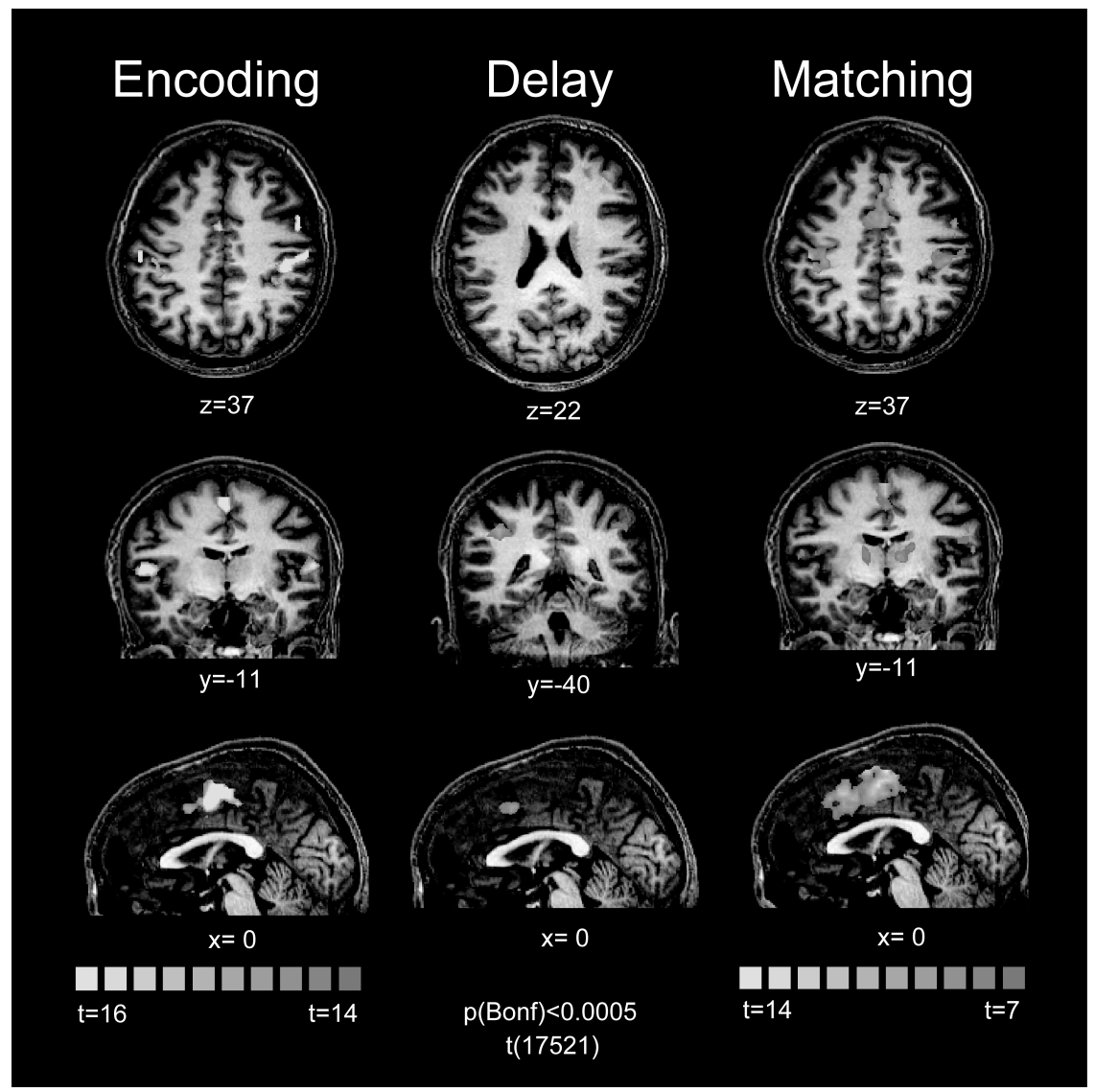

Figure 3. The activation for the encoding, delay and matching phases for the load 1, 2, 3 and 4 trials of the texture working memory task, conjoined for left and right hands. The statistical map from the fixed effects group analysis was thresholded at $\mathrm{t}(17521)=14$ for the encoding phase and at $\mathrm{t}(17521)=7$ for the delay and matching phases. Only those clusters over 250 voxels in size were projected on the anatomical image of an individual participant. Left in the picture corresponds to left in the brain.

After Bonferroni correction, right dlPFC revealed significant effects for the simple load and task contrasts for both hands, and the parametric load contrast for the right hand. For left hand task performance, the parametric load contrast only exceeded the uncorrected threshold. The pre-SMA VOIs only revealed (Bonferroni-corrected) significance for the right hand simple load contrast, which only surpassed the uncorrected threshold for the left hand. The load effects found by performing contrasts on the beta values from the multisubject VOI analysis closely correspond to the ordering of the amplitudes observed in the BOLD percent signal change time courses which were extracted from the clusters found in the group analysis on all runs (figure 4). 


\begin{tabular}{|c|c|c|c|c|c|c|c|c|}
\hline \multirow{3}{*}{$\frac{\text { Table } 1}{\text { Brain region }}$} & \multicolumn{8}{|c|}{ Tactile texture encoding } \\
\hline & \multirow[t]{2}{*}{$\mathrm{BA}$} & \multicolumn{6}{|c|}{ Talairach coordinates } & \multirow{2}{*}{$\begin{array}{l}\text { Number } \\
\text { of Voxels }\end{array}$} \\
\hline & & $\mathrm{x}$ & $\mathrm{y}$ & $\mathrm{z}$ & $\operatorname{sd} x$ & sd y & $\operatorname{sd} z$ & \\
\hline $\mathrm{R}$ postcentral sulcus (including $\mathrm{S} 1$ and $\mathrm{S} 2$ ) & 2 & 50 & -21 & 30 & 9 & 8 & 12 & 4900 \\
\hline R precentral gyrus (vPMA) & 6 & 51 & 4 & 30 & 3 & 4 & 4 & 1125 \\
\hline $\mathrm{R}$ insular cortex & & 39 & -3 & 15 & 3 & 2 & 2 & 301 \\
\hline Medial frontal gyrus (SMA) & 6 & 0 & -2 & 49 & 4 & 7 & 6 & 3934 \\
\hline $\begin{array}{l}\mathrm{L} \text { postcentral gyrus (including S2 and insular } \\
\text { cortex) }\end{array}$ & 43 & -50 & -11 & 12 & 7 & 6 & 5 & 2756 \\
\hline L postcentral sulcus (S1) & 2 & -45 & -27 & 36 & 3 & 4 & 3 & 500 \\
\hline L postcentral gyrus (S1) & 1 & -59 & -19 & 33 & 2 & 3 & 4 & 479 \\
\hline
\end{tabular}

Areas activated in the group analysis over all runs and all 10 participants, identified using the contrast of the texture load 1-4 encoding predictors, conjoined for left and right hand in those participants who performed the texture task with both hands $(N=6)$. Voxel size was interpolated to $1 \mathrm{~mm}^{3}$. Talairach coordinates are reported of the center of gravity (COG) for all clusters over 250 voxels in size, showing significant activation at $\mathrm{t}(17521)=14($ (Bonf) $<0.0005) . B A=B$ rodmann area; $L=$ left; $\mathrm{R}=$ right; $S 1=$ primary somatosensory cortex; $v P M A=$ ventral premotor area; $S M A=$ supplementary motor area; $S 2=$ secondary somatosensory cortex.

\begin{tabular}{|c|c|c|c|c|c|c|c|c|c|c|c|}
\hline Table 2 & & Tactile texture & elay & & & & & & & & \\
\hline \multirow{6}{*}{\multicolumn{2}{|c|}{$\begin{array}{l}\text { Brain } \\
\text { region }\end{array}$}} & \multirow[t]{6}{*}{ participants } & \multicolumn{6}{|c|}{ Talairach coordinates } & \multirow{6}{*}{$\begin{array}{l}\# \\
\text { of } \\
\text { Voxels }\end{array}$} & \multirow{6}{*}{$\begin{array}{l}\mathrm{ma} \\
\mathrm{p} \\
\mathrm{min} \\
\mathrm{t}- \\
\mathrm{val} \\
\mathrm{ue}\end{array}$} & \multirow[t]{6}{*}{ p-value } \\
\hline & & & $\mathrm{x}$ & $\mathrm{y}$ & $\mathrm{z}$ & sd & sd & sd & & & \\
\hline & & & & & & $\mathrm{x}$ & $\mathrm{y}$ & $\mathrm{z}$ & & & \\
\hline & & & & & & & & & & & \\
\hline & & & & & & & & & & & \\
\hline & & & & & & & & & & & \\
\hline \multirow{13}{*}{\multicolumn{2}{|c|}{ 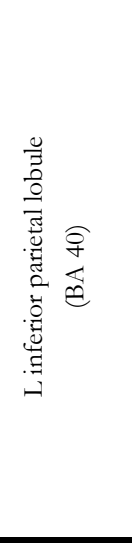 }} & group analysis & -38 & -40 & 36 & 3 & 3 & 2 & 721 & 7 & $\mathrm{p}($ Bonf $)<0.0005$ \\
\hline & & individual & -38 & -50 & 44 & 7 & 11 & 4 & 1049 & & \\
\hline & & VOI mean & & & & & & & & & \\
\hline & & 1 & -32 & -67 & 51 & 6 & 3 & 2 & 389 & 3.5 & $q(F D R)=0.03$ \\
\hline & & 2 & -34 & -37 & 41 & 2 & 1 & 3 & 193 & 4 & $q(F D R)=0.01$ \\
\hline & & 3 & -38 & -43 & 44 & 6 & 3 & 7 & 2407 & 4.3 & $q(F D R)=0.001$ \\
\hline & & 4 & -39 & -40 & 42 & 2 & 3 & 3 & 542 & 2.9 & $q(F D R)=0.05$ \\
\hline & & 5 & -39 & -54 & 47 & 7 & 5 & 7 & 3382 & 2 & $p=0.05$ \\
\hline & & 6 & -49 & -43 & 44 & 2 & 3 & 2 & 465 & 3.5 & $p=0.0005$ \\
\hline & & 7 & -44 & -54 & 46 & 2 & 1 & 1 & 92 & 1.7 & $p=0.10$ \\
\hline & & 8 & -48 & -39 & 40 & 5 & 5 & 4 & 1897 & 2.6 & $p=0.01$ \\
\hline & & 9 & -25 & -61 & 42 & 4 & 2 & 5 & 773 & 1.3 & $p=0.21$ \\
\hline & & 10 & -33 & -66 & 39 & 2 & 3 & 3 & 349 & 2.3 & $p=0.02$ \\
\hline \multirow{5}{*}{\multicolumn{2}{|c|}{ 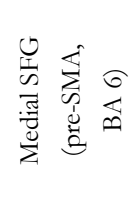 }} & group analysis & -2 & 16 & 42 & 3 & 3 & 2 & 890 & 7 & $\mathrm{p}$ (Bonf) $<0.0005$ \\
\hline & & individual & -4 & 14 & 46 & 5 & 8 & 3 & 304 & & \\
\hline & & VOI mean & & & & & & & & & \\
\hline & & 1 & -5 & 5 & 51 & 5 & 5 & 2 & 828 & 2 & $p=0.05$ \\
\hline & & 2 & 2 & 16 & 49 & 1 & 2 & 1 & 50 & 3.6 & $q(F D R)=0.02$ \\
\hline
\end{tabular}




\begin{tabular}{|c|c|c|c|c|c|c|c|c|c|c|}
\hline & 3 & -14 & 13 & 47 & 2 & 2 & 1 & 114 & 3.9 & $p=0.0001$ \\
\hline & 4 & -4 & 7 & 47 & 3 & 2 & 2 & 597 & 2.9 & $q(\mathrm{FDR})=0.05$ \\
\hline & 5 & 1 & 31 & 43 & 1 & 1 & 2 & 88 & 2 & $p=0.05$ \\
\hline & 6 & 0 & 25 & 44 & 1 & 2 & 1 & 88 & 3.5 & $p=0.0005$ \\
\hline & 7 & -3 & 14 & 44 & 2 & 4 & 3 & 324 & 1.7 & $p=0.10$ \\
\hline & 8 & -5 & 7 & 45 & 2 & 3 & 2 & 451 & 2.6 & $p=0.01$ \\
\hline & 9 & -9 & 13 & 49 & 2 & 2 & 1 & 69 & 1.3 & $p=0.21$ \\
\hline & 10 & -8 & 8 & 41 & 4 & 4 & 2 & 427 & 2.3 & $p=0.02$ \\
\hline \multirow{13}{*}{ 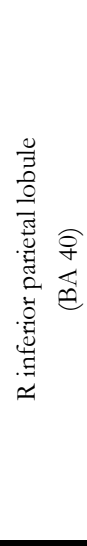 } & group analysis & 43 & -45 & 39 & 2 & 2 & 2 & 382 & 7 & $\mathrm{p}($ Bonf $)<0.0005$ \\
\hline & individual & 42 & -40 & 48 & 6 & 6 & 6 & 210 & & \\
\hline & VOI mean & & & & & & & & & \\
\hline & 1 & 51 & -36 & 55 & 3 & 4 & 1 & 80 & 4.3 & $q(F D R)=0.004$ \\
\hline & 2 & 37 & -42 & 38 & 3 & 2 & 3 & 384 & 3 & $q(F D R)=0.05$ \\
\hline & 3 & 42 & -39 & 49 & 4 & 5 & 5 & 940 & 4.3 & $q(F D R)=0.001$ \\
\hline & 4 & 35 & -41 & 47 & 3 & 4 & 3 & 545 & 2.9 & $q(F D R)=0.05$ \\
\hline & 5 & 46 & -29 & 53 & 2 & 2 & 2 & 303 & 2 & $p=0.05$ \\
\hline & 6 & 51 & -45 & 44 & 1 & 2 & 2 & 102 & 3.5 & $p=0.0005$ \\
\hline & 7 & 40 & -33 & 46 & 2 & 2 & 1 & 88 & 1.7 & $p=0.10$ \\
\hline & 8 & 44 & -44 & 45 & 4 & 3 & 4 & 882 & 2.6 & $p=0.01$ \\
\hline & 9 & 33 & -51 & 43 & 1 & 2 & 1 & 66 & 1.3 & $p=0.21$ \\
\hline & 10 & 42 & -37 & 56 & 4 & 4 & 5 & 836 & 2.3 & $p=0.02$ \\
\hline \multirow{13}{*}{ 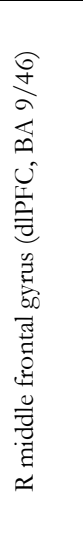 } & group analysis & 39 & 40 & 22 & 2 & 2 & 2 & 214 & 7 & $\mathrm{p}($ Bonf $)<0.0005$ \\
\hline & individual & 39 & 34 & 30 & 9 & 7 & 7 & 210 & & \\
\hline & VOI mean & & & & & & & & & \\
\hline & 1 & 32 & 38 & 28 & 1 & 3 & 2 & 122 & 2 & $p=0.05$ \\
\hline & 2 & 45 & 42 & 22 & 3 & 2 & 2 & 220 & 4 & $q(F D R)=0.01$ \\
\hline & 3 & 43 & 24 & 34 & 2 & 2 & 3 & 207 & 4.3 & $q(F D R)=0.001$ \\
\hline & 4 & 29 & 39 & 32 & 3 & 4 & 4 & 792 & 2.9 & $q(F D R)=0.05$ \\
\hline & 5 & 48 & 31 & 26 & 3 & 3 & 3 & 296 & 2 & $p=0.05$ \\
\hline & 6 & 51 & 26 & 29 & 1 & 1 & 2 & 50 & 3.5 & $p=0.0005$ \\
\hline & 7 & 23 & 29 & 44 & 2 & 1 & 2 & 77 & 1.7 & $p=0.10$ \\
\hline & 8 & 37 & 41 & 23 & 2 & 1 & 2 & 74 & 2.6 & $p=0.01$ \\
\hline & 9 & 37 & 38 & 29 & 1 & 1 & 2 & 67 & 1.3 & $p=0.21$ \\
\hline & 10 & 40 & 30 & 37 & 2 & 2 & 4 & 195 & 2.3 & $p=0.02$ \\
\hline
\end{tabular}

Results for the contrast of texture delay load 1 to 4 predictors against baseline, conjoined for left and right hand. Displayed are the clusters (over 250 voxels) from the group analysis on all runs and all 10 participants (bold), as well as the volumes of interest (VOIs) identified using a single localizer run from each participant for each hand (VOI average across participants and individual VOIs in italics). Participant number 1 to 9 performed both the texture and location task, using their right hand for tactile encoding and matching. Participant 5 to 9 repeated both tasks with their left hand. Participant 10 performed the texture task in the same session, first with the left and then with the right hand. Coordinates of each cluster's centre of gravity (COG) are reported in accordance with the Talairach reference frame. Voxel size was interpolated to $1 \mathrm{~mm}^{3} . L=l e f t ; \mathrm{R}=$ right; $S M A=$ supplementary motor area; $d I P F C=$ dorsolateral prefrontal cortex; $B A=$ Brodmann area; $S F G=$ superior frontal gyrus 


\begin{tabular}{|c|c|c|c|c|c|c|c|c|}
\hline \multirow{3}{*}{$\frac{\text { Table } 3}{\text { Brain region }}$} & \multicolumn{8}{|c|}{ Tactile texture matching and button press } \\
\hline & \multirow[t]{2}{*}{$\mathrm{BA}$} & \multicolumn{6}{|c|}{ Talairach coordinates } & \multirow{2}{*}{$\begin{array}{l}\text { Number } \\
\text { of Voxels }\end{array}$} \\
\hline & & $\mathrm{x}$ & $\mathrm{y}$ & z & $\begin{array}{l}\mathrm{sd} \\
\mathrm{x}\end{array}$ & sd $y$ & $\mathrm{sd} z$ & \\
\hline $\mathrm{R}$ postcentral sulcus (including S1 and S2) & 2 & 49 & -20 & 31 & 8 & 6 & 11 & 3943 \\
\hline R precentral gyrus (vPMA) & 6 & 54 & -2 & 30 & 3 & 5 & 4 & 637 \\
\hline $\mathrm{R}$ anterior insular cortex & & 41 & 16 & 3 & 8 & 4 & 4 & 2852 \\
\hline $\mathrm{R}$ insular cortex & & 40 & -1 & 14 & 3 & 2 & 2 & 316 \\
\hline $\mathrm{R}$ thalamus & & 13 & -6 & 11 & 4 & 8 & 4 & 1779 \\
\hline Medial frontal gyrus (pre-SMA) & 32 & 1 & 7 & 43 & 4 & 13 & 8 & 9948 \\
\hline L thalamus & & -13 & -12 & 10 & 3 & 5 & 4 & 1168 \\
\hline L putamen & & -23 & 1 & 4 & 2 & 4 & 4 & 306 \\
\hline $\mathrm{L}$ anterior insular cortex & & -33 & 18 & 2 & 3 & 3 & 4 & 748 \\
\hline $\mathrm{L}$ insular cortex & & -43 & -2 & 9 & 5 & 4 & 4 & 1316 \\
\hline $\mathrm{L}$ postcentral sulcus $(\mathrm{S} 1)$ & 2 & -42 & -25 & 38 & 4 & 5 & 4 & 1841 \\
\hline L insular cortex / lateral sulcus & & -43 & 11 & 0 & 3 & 2 & 2 & 277 \\
\hline L postcentral gyrus (S2) & $\begin{array}{l}40 / \\
43\end{array}$ & -53 & -16 & 15 & 4 & 3 & 3 & 667 \\
\hline
\end{tabular}

Areas activated in the group analysis over all runs and all 10 participants, identified using the contrast of the texture load 1-4 matching predictors, conjoined for left and right hand $(\mathrm{N}=6)$. Voxel size was interpolated to $1 \mathrm{~mm}^{3}$. Talairach coordinates are reported of the center of gravity (COG) for all clusters over 250 voxels in size, showing significant activation at $t(17521)=7$ (p (Bonf) $<0.0005)$. $B A=$ Brodmann area; $L=$ left $\mathrm{R}=$ right; $S 1=$ primary somatosensory cortex; $v P M A=$ ventral premotor area; $S M A=$ supplementary motor area; $\mathrm{S} 2$ = secondary somatosensory cortex. 


\section{WM related activity}

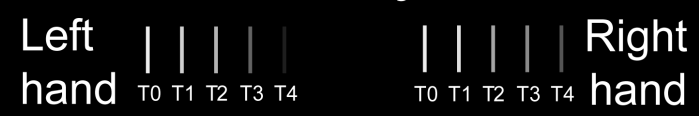

A.

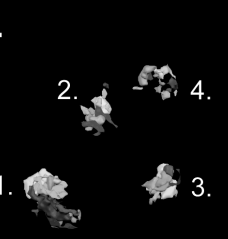

B.

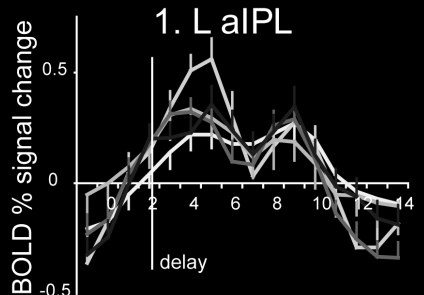

2. pre SMA
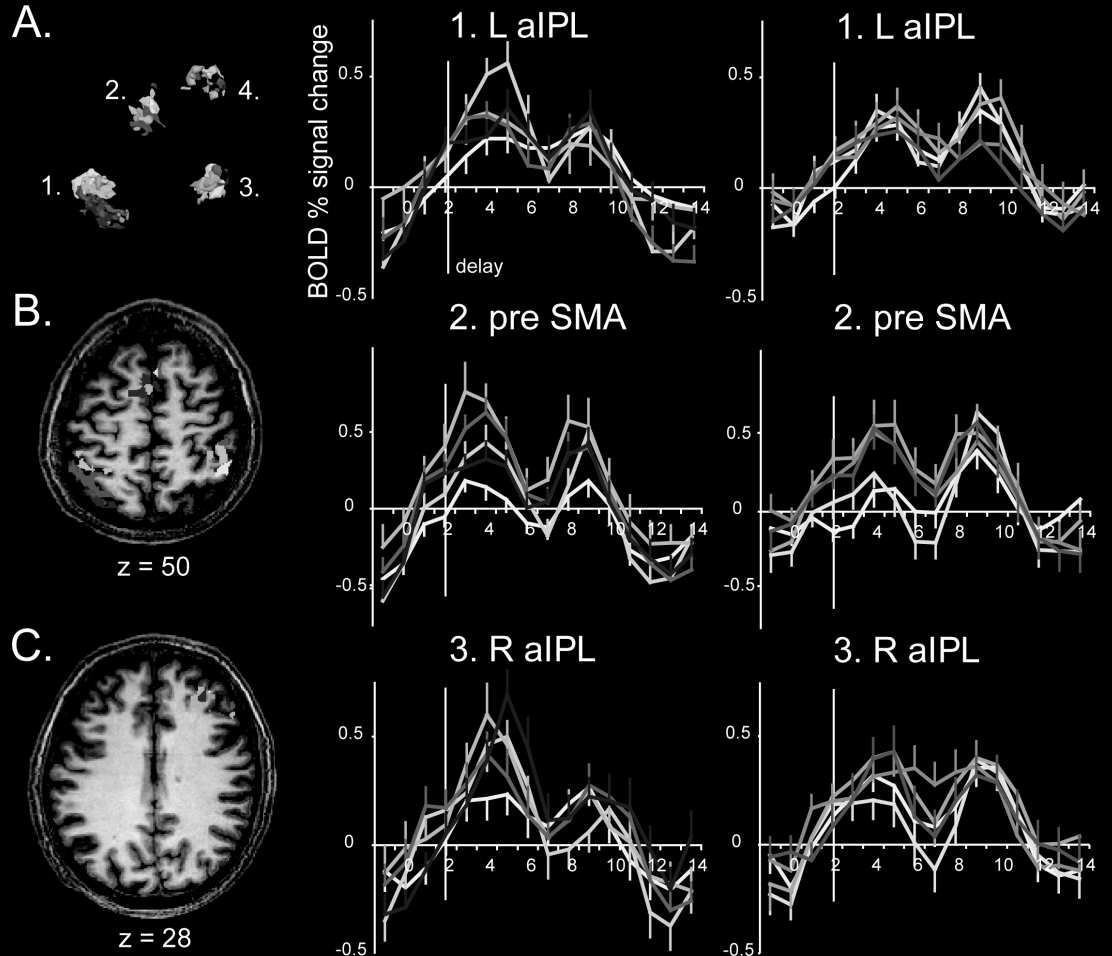

2. pre SMA

3. R alPL

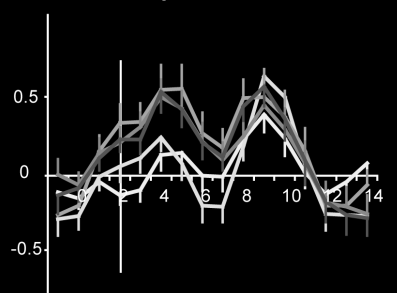

3. $\mathrm{R}$ alPL
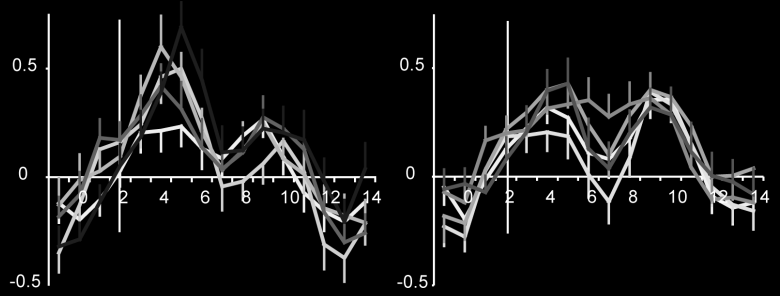

D.

4. R dIPFC
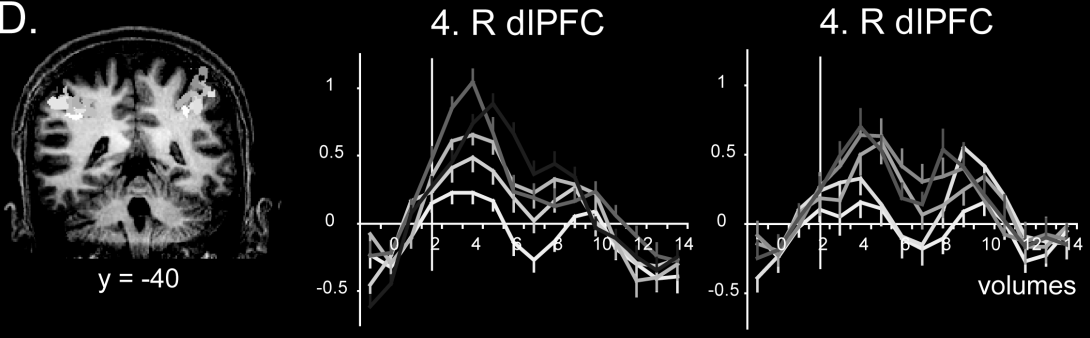

Figure 4. Event-related time courses for each cluster activated in the texture delay, revealed by the group fixed-effects GLM. The trial starts at volume zero, and the start of the delay phase is indicated by a white vertical bar (at volume=2). a: A glass brain, viewed from above, with the group delay clusters displayed in white, as well as the individual VOIs for texture delay load 1, 2, 3 and 4 versus inter trial interval. Each color represents a single participant. 1= left anterior inferior parietal lobule (L aIPL); 2= pre-supplementary motor area (pre SMA); $3=$ right anterior inferior parietal lobule $(\mathrm{R}$ aIPL); $4=$ right dorsolateral prefrontal cortex (R dlPFC). b, c and d: the individual VOIs projected on different slices of a participant's brain. Left in the picture corresponds to left in the brain. For color version, see last pages. 


\section{$\mathrm{R}$ alPL texture delay}

left hand encoding

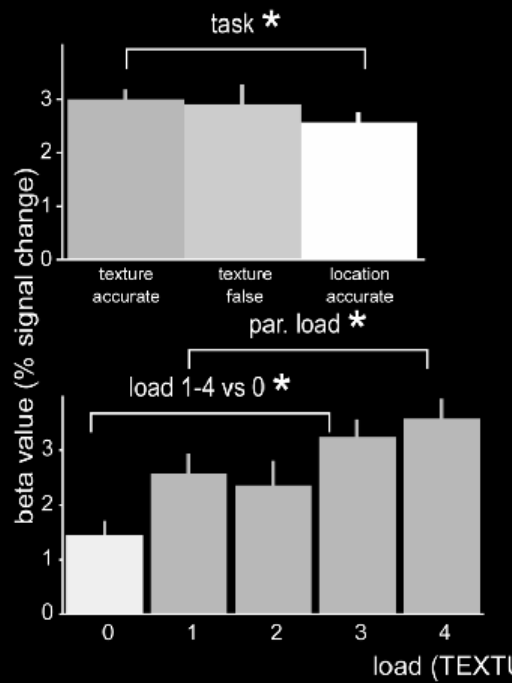

right hand encoding
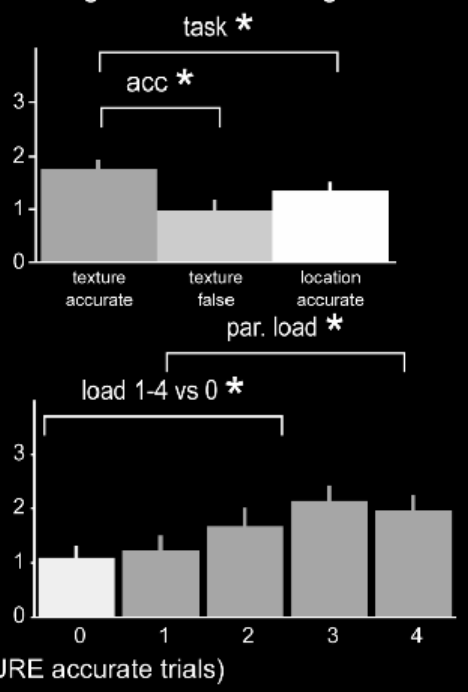

$\mathrm{L}$ alPL texture delay

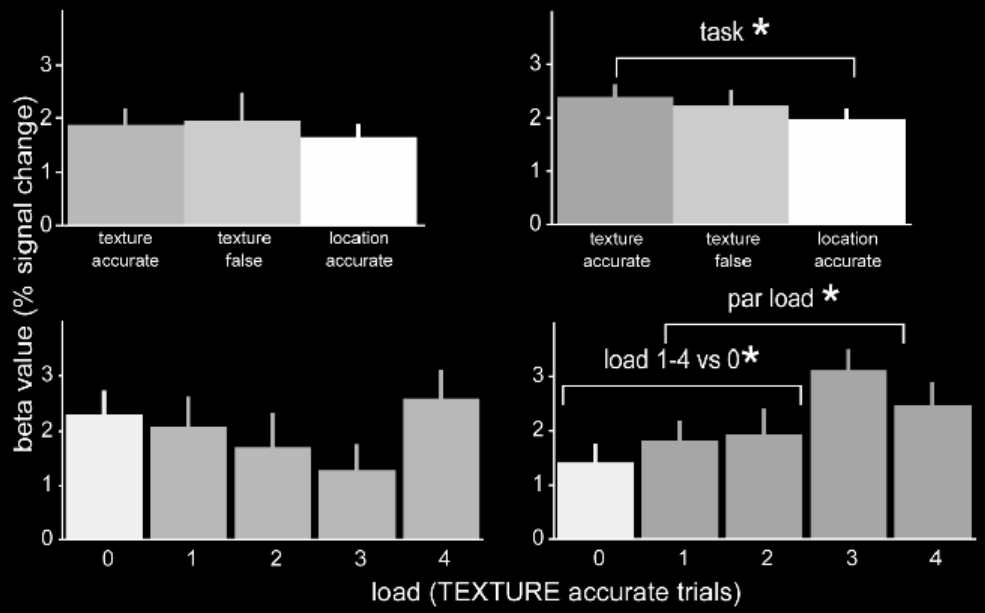

Figure 5. The beta values for the analysis on the individual texture delay VOIs for right and left aIPL (right hand: texture $\mathrm{N}=10$, location $\mathrm{N}=9$; left hand: texture $\mathrm{N}=6$, location $\mathrm{N}=5$ ). The significance of the Task, Load 1-4 versus Load 0, Parametric Load (par. load) and accuracy (acc.) contrasts are indicated by asterisks, single for significance at $\mathrm{p}$ (uncorrected) $<0.05$ and double for $\mathrm{p}<0.00625$ (Bonferroni corrected). 


\section{Discussion}

The current study investigated the neural substrate for tactile texture working memory, using a delayed match-to-sample task, in which either the texture or location of sandpaper stimuli had to be maintained in working memory during a 10 to $12 \mathrm{sec}$ delay. Memory load was varied by changing the number of stimuli from 0 to 4 .

We found activation in bilateral anterior IPL, pre-SMA and right dIPFC during the delay of the tactile texture working memory task. Right aIPL and right dlPFC showed enhanced activation with increasing tactile texture loads. Moreover, the activation level in these areas was specific for tactile texture working memory, showing a significantly higher activation level than during the delay of the location task. While the task and load effects in right dIPFC and right aIPL were independent of the hand used for encoding and matching, the left aIPL activation pattern was not. Although left aIPL did not show task or load modulation for the left hand, this area revealed significantly higher responses for higher texture loads as well as a higher activation for the texture task than for the location task when the right hand was used. However, for the right hand, only the activation level in right aIPL was predictive for subsequent matching accuracy. Pre-SMA did not show increasing responses for higher workloads, nor for texture versus location matching. Taken together, these results indicate a significant role for right aIPL and right dIPFC in tactile texture working memory.

\section{Task performance}

Previous behavioural studies revealed left hand advantages in both speed and accuracy for non-verbal tactile tasks (Milner and Taylor, 1972; Kumar, 1977; Dodds, 1978; Zuidhoek et al., 2005). Despite the lateralization of the parametric load effect and task difference for the left hand in the current study, there was no difference in delayed match-to-sample accuracy between left and right hand. The hand-independence of the behavioral accuracy could be brought about by inter-hemispheric compensation for right hand performance during the tactile working memory delay. Zuidhoek et al. (2005) found that right hand accuracy increased to the level of the left hand after a ten second delay, possibly caused by inter-hemispheric compensation processes during the delay. This might be reflected by the additional load and task effects in left aIPL, which were only observed for task performance with the right hand.

Location matching showed a significantly higher percentage correct compared to texture matching, indicating that location matching was easier. While accuracy for texture matching remained stable across different loads, location matching showed a drop in performance at load 3. This might be explained by the interference of a reversal strategy, in which the number of unfilled stimulus locations is remembered.

At load 4, location matching is trivial: since all locations were filled, the probe would always be a match. Therefore, the percentage correct for location load 4 was expected to be close to $100 \%$. However, we found that performance for this condition was characterized by a much lower average of only $74 \%$ correct, with a relatively high standard error. This result might be explained by the fact that some participants reacted to location load 4 ("all filled") in a similar way as to load 0 ("all empty"), by randomly pressing a response button. This explanation is also in accordance with self-reports given by the participants after the study. 


\section{$\mathrm{R}$ dIPFC texture delay}

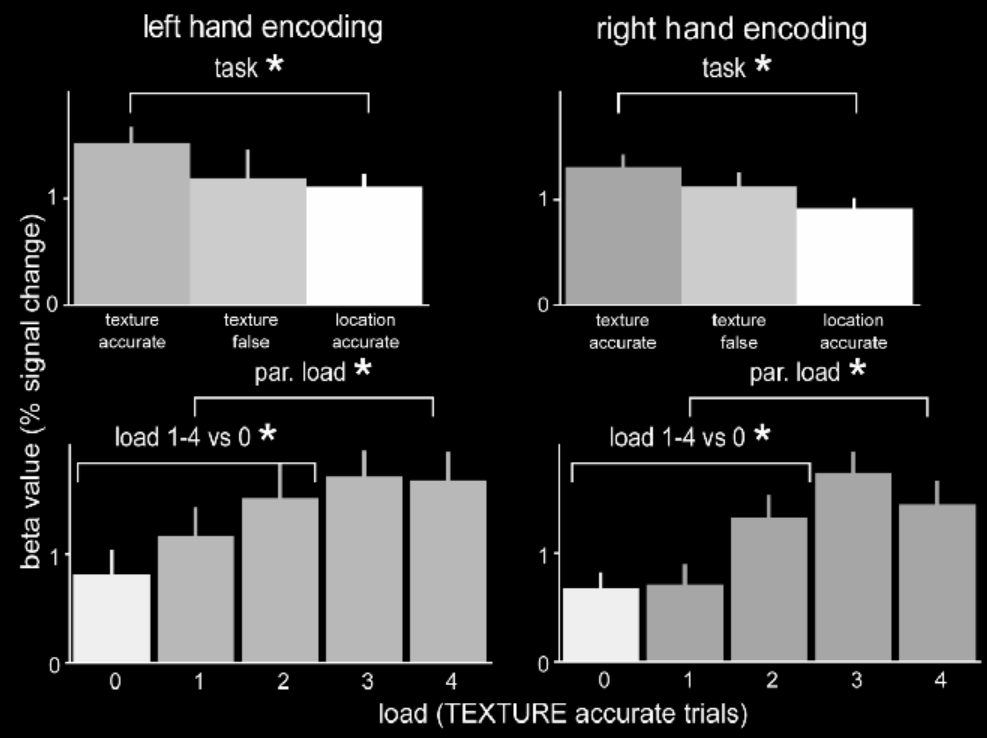

\section{pre SMA texture delay}

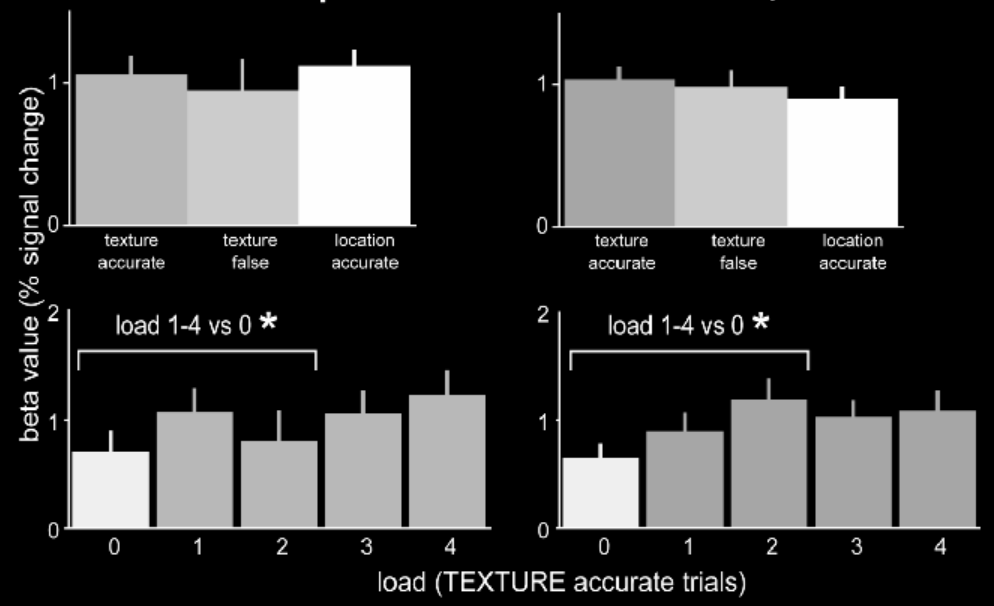

Figure 6. The beta values for the analysis on the individual texture delay VOIs for right dIPFC and bilateral pre-SMA (right hand: texture $\mathrm{N}=10$, location $\mathrm{N}=9$; left hand: texture $\mathrm{N}=6$, location $\mathrm{N}=5$ ). The significance of the Task, Load 1-4 versus Load 0, Parametric Load (par. load) and accuracy (acc.) contrasts are indicated by asterisks, single for significance at $\mathrm{p}$ (uncorrected) $<0.05$ and double for $\mathrm{p}<0.00625$ (Bonferroni corrected). 


\section{Texture encoding and matching}

Tactile texture encoding engaged bilateral primary and secondary somatosensory cortices, bilateral insular cortices, medial SMA and right vPMA. This activation corresponds to activation found in previous imaging studies of tactile discrimination (e.g. Roland et al., 1998; Stoeckel et al., 2004; Zhang et al., 2005), and to the network for tactile memory proposed by Burton and Sinclair (2000). Activation of right vPMA was previously found by Ehrsson et al. (2000), when comparing precision versus power grips, and might correspond to area F5 in macaques, involved when picking up small objects using index finger and thumb in a precision grip configuration. We propose that this region might be specifically involved in fine motor coordination of the hand configuration needed for accurate exploration of tactile stimuli.

The statistical contrast of texture versus location encoding revealed a single cluster in secondary somatosensory cortex which was significantly more activated during texture encoding. Roland et al. (1998) showed that secondary somatosensory cortex is specifically involved in tactile roughness discrimination. Therefore, the activation of this area in the texture task might confirm that participants primarily focused on processing stimulus roughness in the texture task.

Feeling the probe and pressing the response button with the contralateral hand activated regions that largely overlapped with those involved in stimulus encoding, and included bilateral primary and secondary somatosensory cortices, bilateral insular cortices, medial (pre-)SMA and right vPMA. Due to the shorter length of the matching compared to the encoding phase in the texture task (only one probe had to be felt, as opposed to four potential stimulus positions during encoding), the activation level during texture matching was lower that during texture encoding. When the different thresholds used for encoding and matching phase are disregarded, the matching phase is characterized by additional clusters in bilateral thalamus, left putamen and anterior insula, as well as the extension of the SMA cluster into pre-SMA.

The insula forms part of a pathway which relays somatosensory information arriving from S1 via S2 to the limbic structures of the temporal lobe (amygdala and hippocampus), potentially subserving tactile recognition and recall. It has been suggested that the insular cortex participates in tactile memory like the inferotemporal cortex in visual memory (Augustine, 1996; Burton and Sinclair, 2000). The anterior insula has also been described as a motor association area.

\section{Texture delay}

In the delay, a parieto-frontal network was activated, including bilateral aIPL, right dIPFC and pre-SMA. These areas were characterized by differential responses to task, accuracy and two load contrasts.

Pre-supplementary motor area (pre-SMA) Significantly higher activation was found in preSMA when comparing the texture delay predictors for load 1 to 4 to the load 0 predictor. In previous visual working memory studies, pre-SMA showed sustained activation in the delay of both spatial and non-spatial tasks. It was functionally classified as a prefrontal area, supporting modality- and effector-independent sensory-motor associations (Picard and Strick, 2001) and the selection of action sets (Rushworth et al. 2004). It is suggested to reflect "a state of preparedness for selection of a motor response 
based on the information held online" (Petit et al., 1998), which might be independent of the amount or type of information. This interpretation corresponds with our observations that pre-SMA did not reveal significant increases for higher workloads, nor for the texture compared to location delay.

Inferior parietal lobule / intra-parietal sulcus The aIPL region found in the current study was located on the lateral bank close to the fundus of the intraparietal sulcus. It might correspond to the human equivalent of the macaque anterior intraparietal sulcus (AIP). Human AIP is involved in the (crossmodal) transformation of object information for hand preshaping in grasping, and also during object holding and manipulation (Binkofski et al., 1999; Ehrsson et al., 2000; Grefkes et al., 2002; Culham et al. 2003; Frey et al., 2005). A previous study in right-handed participants found that imagining roughness of common objects induced higher activation of left and right intraparietal sulcus (IPS) than imagining the geometric properties shape and size or other material properties like hardness and temperature (Newman et al., 2005). The activation in these IPS clusters, posterior to the current findings in aIPL, were interpreted as the neural substrate of visual imagery for material properties. A more posterior cluster in right angular gyrus showed higher activation for passive microgeometric (line spacing) as compared to passive macrogeometric (grating orientation) discriminations (Sathian et al., 1997; Zhang et al., 2005). We propose that the current locus of activity in right aIPL might subserve the maintenance of surface characteristics, important for the fine calibration of forces applied to thumb and index finger in precision grips; this would be consistent with the observation by Ehrsson et al. (2000) that right anterior IPS was more active for right-handed precision versus power grips, in which the whole hand is curved around the object. They suggested that this right-sided activity reflected the additional demands on somatosensory control and sensori-motor processing in precision versus power grips.

In the present study, the left parietal activation was more extended than the righthemispheric activation for the group VOI and the individual VOIs averaged over participants. Nevertheless, the smaller right aIPL cluster showed sensitivity to load increases, as well as a texture preference for both left and right-handed task performance. Left aIPL did not show significance for the task and parametric load contrasts when the left hand was used, but these contrasts did reach significance in this area for performance with the right hand. However, for the right hand, only the ipsilateral right aIPL activation level was predictive of a subsequent accurate matching of tactile texture working memory. Therefore, our data suggest a specific importance of the right parietal cortex for maintenance of fine tactile surface textures. It could be that both left and right aIPL can maintain a representation of tactile surfaces, but additional right aIPL resources might be recruited when workload is increased. Correspondingly, the event-related time courses and beta values in figures 4 and 5 show that for the right hand, right aIPL is recruited in addition to left aIPL for maintenance of more than one texture. Stoeckel et al. (2004) found left anterior superior parietal lobule (aSPL) to support maintenance of the oblongness of a single parallelepiped explored with the right hand. During right hand exploration and discrimination of a second parallelepiped, right aSPL became involved, but only for parallelepipeds above the discrimination 
threshold. The right aIPL activation could thus be interpreted to react to the increased workload during discrimination.

The task and load-dependent modulation of the current left aIPL activity for the right hand might reflect ongoing inter-hemispheric transfer of information, communicating the result of computations in the right aIPL to the left aIPL. It might also be that tactile texture working memory for microspatial stimuli in right peri-personal and somatosensory space recruits bilateral parietal areas, analogous to the bilateral recruitment for (visuo-)spatial processing of left-hemifield extrapersonal space (Coghill et al., 2001).

Right dorsolateral prefrontal cortex Right dlPFC showed a higher response for texture versus location working memory, load versus non-load (load 0) trials and load dependent increasing responses for higher workloads. Regions in dorsolateral prefrontal cortex have been argued to contribute to executive control processes in working memory, e.g. updating, monitoring and manipulation of new information, instead of storing memory representations per se (e.g Smith \& Jonides, 1999; Petrides, 2000; Hillary et al., 2006; Blumenfeld and Ranganath, 2006). Activity in dlPFC was found in previous studies of tactile short term memory in humans (Klingberg et al. 1996; Numminen et al. 2004) and non-human primates (Romo et al. 1999). In addition, dlPFC was proposed to be involved in skilled force production for precision grips, in which isometric grip force has to be adjusted to the object's weight and surface characteristic (Ehrsson et al., 2000). Right hemispheric dlPFC might be of special importance in cognitive tasks challenging the limits of working memory capacity (Hillary et al. 2006). The current tactile texture matching task fits this description, in using relatively uncommon nonobject and non-verbal tactile stimuli.

\section{Lateralization of tactile working memory}

Several behavioral experiments testing for speeded tactile (non-verbal) discriminations have found superior left hand performance (Milner and Taylor, 1972; Kumar, 1977; Dodds, 1978; Zuidhoek et al., 2005). Some studies only found right-hemisphere dominance for male participants (e.g. Genetta-Wadley and Swiskey-Sacchetti, 1990). This might be due to the fact that, on average, the male brain is more lateralized than the female brain (Kimura, 1996; Toga and Thompson, 2003). The current study only tested male participants, eliminating the effects of gender on functional specialization of the cerebral hemispheres. It is very well possible that the right-lateralization of tactile texture working memory is less pronounced in females. Nevertheless, testing both males and females, Harada et al. (2004) found asymmetrical right hemisphere activation in right dIPFC and intraparietal sulcus during a passive tactile shape discrimination task. Knecht et al. (1996) observed bilateral impairments in four out of five righthanded male participants with focal right parietal lesions, and suggested that the right parietal cortex has a bilateral integrative function in the processing of spatiotemporally coded somesthetic information, independent from attentional or general cognitive factors. Coghill et al. (2001) reported right lateralized activation for both innocuous and painful stimulation on either side of the body, suggesting that stimuli arising from the body surface are processed by right lateralized systems, in part analogous to those processing information from extrapersonal visual or auditory space. The left parietal activation in the present study might reflect specific attention related to limb move- 
ment (Rushworth et al., 2001). Right parietal cortex activation, in contrast, has been implied in top-down visuo-spatial orienting (Corbetta and Shulman, 2002). Although participants might have oriented towards the location where the stimuli would be presented, the demands on spatial attention were equal in the different load conditions. Hence, the load modulation of the right parietal cortex most likely reflects increasing demands on tactile working memory, rather than increasing demands on spatial attention.

\section{Comparison to previous studies of active tactile working memory and lesion studies}

As opposed to previous studies of active tactile working memory (Kaas et al., in press; chapter 5; Stoeckel et al. 2003; Ricciardi et al., 2005), the tactile texture working memory delay in the current study did not recruit occipital or occipitotemporal regions, e.g. lateral occipital cortex or fusiform gyrus, traditionally associated with visual object perception and imagery. After extraction of tactile texture information by the primary and secondary somatosensory cortices, the tactile sensory trace might have been converted to a more abstract sensorimotor-related representation, which appeared to be maintained in a relatively anterior region of the inferior parietal lobule. A previous study of delayed haptic orientation matching engaged areas in parieto-occipital cortex (Kaas et al., in press; chapter 5). In both monkeys and humans, parieto-occipital regions and caudal IPS were shown to be specifically involved in the coding of 3D geometric object features, while anterior IPS responded most when the hand had to be shaped for object grasping (Shikata et al, 2003; Sakata et al. 2005). Lesion studies showed that parietal lesions resulted in tactile apraxia, i.e. an isolated disturbance of transitive (object-related) movements with preserved intransitive movements. Astereognosia (inability to make 2D/3D shape and form discriminations) was most marked for tactile apraxics with posterior parietal lesions (Binkofski et al. 2001). By varying workload and task instruction, the current experiment identified and characterized the cortical areas involved in the maintenance of tactile texture information. Our results provide evidence for the existence of specialized areas supporting the maintenance of specifically tactile object features. Whether the neural substrate for tactile texture working memory is truly distinct from the neural correlates maintaining 3D information or other tactile object properties, remains to be tested in future experiments. These experiments will need to meet the challenge to directly compare maintenance of different object features, while carefully controlling for differences in exploratory movement sequences.

\section{Conclusion}

The current study revealed activity in a parieto-frontal network including bilateral aIPL, pre-SMA and right dIPFC, supporting the maintenance of tactile texture information in a delayed match-to-sample task. Based on the modulation of activation by task instruction, workload, and response accuracy observed in right aIPL and dlPFC, we propose that these areas subserve processes specific to tactile texture working memory. While right dIPFC might support cognitive control mechanisms needed to 
meet the processing requirements for increasing workload, right aIPL might be involved in the representation of tactile surface features. Our results stress both the supramodal nature of parieto-frontal networks involved in cognitive control, as well as the particular importance of the anterior inferior parietal lobe for higher order tactile processing.

\section{Acknowledgement}

We thank Nadia Mueller, Anneke Hinzen and the technical personnel of the Universtity Maastricht for their kind assistance. In addition, we thank Joel Reithler, Judith Peters and Alexander Sack for their helpful comments.

\section{References}

Amedi A, Malach R, Hendler T, Peled S, Zohary E (2001) Visuo-haptic object-related activation in the ventral visual pathway. Nat Neurosci 4: 324-330.

Amedi A, Jacobson G, Hendler T, Malach R, Zohary E (2002) Convergence of visual and tactile shape processing in the human lateral occipital complex. Cereb Cortex 12: 1202-1212.

Augustine JR (1996) Circuitry and functional aspects of the insular lobe in primates including humans. Brain Res Brain Res Rev 22: 229-44.

Binkofski F, Buccino G, Posse S, Seitz RJ, Rizzolatti G, Freund H-J (1999) A fronto-parietal circuit for object manipulation in man: evidence from an fMRI-study. Eur J Neurosc 11: 3276-3286.

Binkofski F, Kunesch E, Classen J, Seitz RJ, Freund H-J (2001) Tactile apraxia: unimodal apractic disorder of tactile object exploration associated with parietal lobe lesions. Brain (124): 132-144.

Blumenfeld RS, Ranganath C (2006) Dorsolateral prefrontal cortex promotes long-term memory formation through its role in working memory organization. J Neurosc 26: 916-925.

Bodegård A, Geyer S, Grefkes C, Zilles K, Roland P (2001) Hierarchical Processing of Tactile Shape in the Human Brain. Neuron 31: 317-328.

Bohlhalter S, Fretz C, Weder B (2002) Hierarchical versus parallel processing in tactile object recognition: a behavioural-neuroanatomical study of aperceptive tactile agnosia. Brain 125: 2537-2548.

Bonda E, Petrides M, Evans A (1996) Neural systems for tactual memories. J Neurophysiol 75: 1730-1737.

Boynton GM, Engel SA, Glover GH, Heeger DJ (1996) Linear Systems Analysis of Functional Magnetic Resonance Imaging in Human V1. J. Neurosci 16: 4207 - 4221.

Burton H, Sinclair RJ (2000) Attending to and remembering tactile stimuli: A review of brain imaging data and single-neuron responses. J Clin Neurophysiol 17: 575-591.

Bushnell EW, Baxt C (1999) Children's haptic and cross-modal recognition with familiar and unfamiliar objects. J Exp Psychol Hum Percept Perform 25:1867-1881.

Coghill RC, Gilron I, Iadarola M (2001) Hemispheric lateralization of somatosensory processing. J Neurophysiol 85: 2602-2612.

Constantinidis C, Procyk E (2004) The primate working memory networks. Cogn Affect Behav Neurosci 4: 444-465.

Corbetta M, Shulman GL (2002) Control of goal-directed and stimulus-driven attention in the brain. Nat Rev Neurosci 3: 201-215. 
Culham J, Danckert S, DeSouza J, Gati J, Menon R, Goodale M (2003) Visually guided grasping produces fMRI activation in dorsal but not ventral stream brain areas. Exp Brain Res 153: 180-189.

Deibert E, Kraut M, Kremen S, Hart J Jr. (1999) Neural pathways in tactile object recognition Neurology 52: 1413-1417.

Dodds AG (1978) Hemispheric differences in tactuo-spatial processing. Neuropsychologia 16:247-250. Easton RD, Srinivas K, Greene AJ (1997) Do vision and haptics share common representations? Implicit and explicit memory within and between modalities. J Exp Psychol Learn Mem Cogn 23: 153-163.

Ehrsson HH, Fagergren A, Jonsson T, Westling G, Johansson RS, Forssberg H (2000) Cortical activity in precision- versus power-grip tasks: an fMRI study. J Neurophy siol (83): 528-536.

Frey SH, Vinton D, Norlund R, Grafton ST (2005) Cortical topography of human anterior intraparietal cortex active during visually guided grasping. Cog Brain Res 23: 397-405.

Genetta-Wadley A, Swirsky-Sacchetti T. (1990) Sex differences and handedness in hemispheric lateralization of tactile-spatial functions. Percept Mot Skills 70: 579-590.

Goebel R, Esposito F, Formisano E (2006) Analysis of FIAC data with BrainVoyager QX: From singlesubject to cortically aligned group GLM analysis and self-organizing group ICA. Hum Brain Mapp 27: 392401.

Grefkes C, Weiss P, Zilles K, Fink G (2002) Crossmodal processing of object features in human anterior intraparietal cortex: An fMRI study implies equivalencies between humans and monkeys. Neuron 35: 173184.

Guest S, Spence C (2003) Tactile dominance in speeded discrimination of textures. Exp Brain Res 150: 201-207.

Harada T, Saito DN, Kashikura K, Sato T, Yonekura Y, Honda M, Sadato N (2004) Asymmetrical neural substrates of tactile discrimination in humans: a functional magnetic resonance imaging study. J Neurosci 24: 7524-7530.

Heller MA (1989) Texture perception in sighted and blind observers. Percept Psychophys 45: 49-54.

Hillary FG, Genova HM, Chiaravalloti ND, Rypma B, DeLuca J (2006) Prefrontal activation of working memory performance in brain injury and disease. Hum Brain Mapp [Epub ahead of print] DOI 10.1002/hbm.20226.

Hiscock M, Inch R, Hawryluk J, Lyon PJ, Perachio N (1999) Is there a sex difference in human laterality? III. An exhaustive survey of tactile laterality studies from six neuropsychology journals. J Clin Exp Neuropsychol 21:17-28.

Jones B, O'Neil S (1985) Combining vision and touch in texture perception. Percept Psychophys 37: 66-72.

Kaas AL, van Mier HI, Goebel R. The neural correlates of human working memory for haptically explored object orientations. Cereb Cortex. In Press.

Kimura D (1996) Sex, sexual orientation and sex hormones influence human cognitive function. Curr Opin Neurobiol 6: 259-263.

Klatzky RL, Lederman S, Reed C (1987) There's more to touch than meets the eye : the salience of object attributes for haptics with and without vision. J Exp Psychol Gen 116: 356-369.

Klatzky RL, Lederman SJ, Matula DE (1993) Haptic exploration in the presence of vision. J Exp Psychol Hum Percept Perform 19: 726-743.

Klingberg T, Kawashima R, Roland PE (1996) Activation of multi-modal cortical areas underlies shortterm memory. Eur J Neurosci 8: 1965-1971.

Knecht S, Kunesch E, Schnitzler A (1996) Parallel and serial processing of haptic information in man: Effects of parietal lesions on sensorimotor hand function. Neuropsychologia 34: 669-687.

Koch C, Fuster JM (1989) Unit activity in monkey parietal cortex related to haptic perception and temporary memory. Exp Brain Res 76: 292-306. 
Kumar S (1977) Short term memory for a nonverbal tactual task after cerebral commissurotomy. Cortex 13: 55-61.

Merabet L, Thut G, Murray B, Andrews J, Hsiao S, Pascual-Leone A (2004) Feeling by sight or seeing by touch? Neuron 42: 173-179.

Milner B, Taylor L (1972) Right-hemisphere superiority in tactile pattern-recognition after cerebral commissurotomy: evidence for nonverbal memory. Neuropsychologia 10: 1-15.

Newman SD, Klatzky RL, Lederman SJ, Just MA (2005) Imagining material versus geometric properties of objects: an fMRI study. Cog Brain Res 23: 235-246.

Numminen J, Schurmann M, Hiltunen J, Joensuu R, Jousmaki V, Koskinen SK, Salmelin R, Hari R (2004) Cortical activation during a spatiotemporal tactile comparison task. Neuroimage 22: 815-821.

Oldfield RC (1971) The assessment and analysis of handedness: The Edinburgh inventory. Neuropsychologia 9: 97-113.

Petit L, Courtney SM, Ungerleider LG, Haxby JV (1998) Sustained activity in the medial wall during working memory delays. J Neurosci 18: 9429 - 9437.

Petrides M (2000) Dissociable roles of mid-dorsolateral prefrontal and anterior inferotemporal cortex in visual working memory. Neurosci 20: 7496 - 7503.

Picard D (2006) Partial perceptual equivalence between vision and touch for texture information. Acta Psychol 121: 227-248.

Picard N, Strick PL (2001) Imaging the premotor areas. Curr Opin Neurobiol 11: 663-672.

Pietrini P, Furey ML, Ricciardi E, Gobbini MI, Wu WH, Cohen L, Guazzelli M, Haxby JV (2004) Beyond sensory images: Object-based representation in the human ventral pathway. Proc Natl Acad Sci U S A 101:5658-5663.

Prather SC, Votaw JR, Sathian K (2004) Task-specific recruitment of dorsal and ventral visual areas during tactile perception. Neuropsychologia 42: 1079-1087.

Randolph M, Semmes J (1974) Behavioral consequences of selective subtotal ablations in the postcentral gyrus of Macaca mulatto. Brain Res 70: 55-70.

Reales JM, Ballesteros S (1999) Implicit and explicit memory for visual and haptic objects: cross-modal priming depends on structural descriptions. J Exp Psychol Learn Mem Cogn 25: 644-663.

Ricciardi E, Bonino D, Gentili C, Sani L, Pietrini P, Vecchi T (2005) Neural correlates of spatial working memory in humans: a functional magnetic resonance imaging study comparing visual and tactile processes. Neuroscience [Epub ahead of print] doi:10.1016/j.neuroscience.2005.08.045.

Roland PE, O'Sullivan B, Kawashima R (1998) Shape and roughness activate different somatosensory areas in the human brain. Proc Natl Acad Sci U S A. 95: 3295-3300.

Romo R, Brody CD, Hernandez A, Lemus L (1999) Neuronal correlates of parametric working memory in the prefrontal cortex. Nature 399: 470-473.

Rushworth MFS, Krams M, Passingham RE (2001) The Attentional Role of the left parietal cortex: the distinct lateralization and localization of motor attention in the human brain. J Cog Neurosci 13: 698-710.

Rushworth MFS, Walton ME, Kennerley SW, Bannerman DM (2004) Action sets and decisions in the medial frontal cortex. Trends Cogn Sci 8: 410-417.

Sadato N, Ibañez V, Deiber M-P, Hallett M (2000) Gender difference in premotor activity during active tactile discrimination. Neuroimage 11: 532-540.

Sakata H, Tsutsui K-I, Taira M (2005) Toward an understanding of the neural processing for 3D shape perception. Neuropsychologia 43: 151-161.

Sathian K, Zangaladze A, Hoffman JM, Grafton ST (1997) Feeling with the mind's eye. Neuroreport 8: 3877-3881. 


\section{4 | Chapter 6}

Shikata E, Hamzei F, Glauche V, Koch M, Weiller C, Binkofski F, Buchel C (2003) Functional properties and interaction of the anterior and posterior intraparietal areas in humans. Eur J Neurosci 17: 1105-1110. Smith EE, Jonides J (1999) Storage and Executive Processes in the Frontal Lobes. Science 283: 1657-1661. Stoeckel MC, Weder B, Binkofski F, Buccino G, Shah NJ, Seitz RJ (2003) A fronto-parietal circuit for tactile object discrimination: An event-related fMRI study. Neuroimage 19: 1103-1114.

Stoeckel MC, Weder B, Binkofski F, Choi H-J, Amunts K, Pierperhoff P, Shah NJ, Seitz RJ (2004) Left and right superior parietal lobule in tactile object discrimination. Eur J Neurosc 19: 1067-1072.

Stoesz MR, Zhang M, Weisser VD, Prather SC, Mao H, Sathian K (2003) Neural networks active during tactile form perception: common and differential activity during macrospatial and microspatial tasks. Int $\mathrm{J}$ Psychophysiol 50: 41-49.

Toga AW, Thompson PM (2003) Mapping brain asymmetry. Nat Rev Neurosci 4: 37-48.

Zangaladze A, Epstein CM, Grafton ST, Sathian K (1999) Involvement of visual cortex in tactile discrimination of orientation. Nature 401: 587-590.

Zhang M, Mariola E, Stilla R, Stoesz M, Mao H, Hu X, Sathian K (2005) Tactile Discrimination of Grating Orientation: fMRI Activation Patterns. Hum Brain Mapp 25: 370 -377.

Zhou YD, Fuster JM (1996) Mnemonic neuronal activity in somatosensory cortex. Proc Natl Acad Sci U S A 93: 10533-10537.

Zuidhoek S, Kappers AML, Postma A (2005) Effects of hand orientation and delay on the verbal judgment of haptically perceived orientation. Perception 34: 741-755. 


\section{Summary and Conclusions}

$\mathbf{T}$

he current project investigated the neural bases of tactile object manipulation, using behavioral measures and functional magnetic resonance imaging (fMRI), a noninvasive technique measuring task-related changes in brain activity. Two main research questions were addressed. Firstly, we investigated the reference frames used when performing spatial matching tasks by active touch (haptics). The results of this investigation were reported in chapters 2, 3 and 4. Secondly, we used fMRI to study which brain areas are involved in touching and remembering large or small scale object features, i.e. (micro spatial) roughness or (macro spatial) orientation, for a few seconds. The findings from the fMRI experiments were presented in chapters 5 and 6.

\section{Reference frames in haptic spatial matching}

Accurate representations of peripersonal space (the space around our bodies) seem indispensable for successful object manipulation. However, haptic spatial representations were found to suffer from systematic egocentric errors. These errors are especially striking in haptic orientation matching tasks, in which participants turn a test bar to the orientation of a reference bar, using only active touch. In parallel matching, the instruction is to turn the test bar to a parallel orientation (/ /). In contrast, in mirror matching, the test bar is turned to an orientation which is the mirror image of the reference bar $(/ \backslash)$ in the midsagittal plane of the body. In principle, veridical parallel matching requires an allocentric reference frame, independent of body position, preserving the spatial relations in extrapersonal space. Conversely, mirror matching can be performed accurately in both allo- and egocentric reference frames. In an egocentric reference frame spatial representations are fixed to the body.

In previous studies large systematic errors have been observed in haptic parallel matching at inter manual distances of $60 \mathrm{~cm}$ or more; the error size and direction were correlated to the difference between the natural orientation of the hand at the location of the reference and test bar. Yet, close to veridical performance has been observed in haptic mirror matching. Based on these results, a common 'intermediate' reference frame has been proposed for parallel and mirror matching, built from a participantdependent, weighted average of an egocentric, hand-centered frame, and an allocentric, world-based frame. On the other hand, it has also been argued that parallel matching promotes the use of an extrinsic, visual reference frame, whereas haptic mirror matching endorses an intrinsic, hand-centered frame. 
The experiments described in chapters 1, 2, and 3 further explored the nature of the reference frames dominating haptic parallel and mirror matching, studying the effect of different task manipulations on matching accuracy in right-handed participants. In particular, the influence of inter manual distance, gender, visual information, body posture and hand used for exploration and matching was investigated, based on the rationale that when one of these parameters changed matching accuracy, it would indicate that the reference frame underlying performance is dependent on that parameter. All participants were right-handed. Unless mentioned otherwise, the reference bar was explored with the left hand for 1.5 seconds, and the test bar was turned with the right hand within 2 seconds. Reference and test bars were located at symmetrical positions with respect to the body-midsagittal plane, and a $60 \mathrm{~cm}$ inter manual distance was used.

Chapter 2 compared haptic matching accuracy of right-handed male and female participants, and reported the effects induced by variation of inter manual distance $(30 \mathrm{~cm}$ or $60 \mathrm{~cm})$ and delay $(0.5,5$ or 10 seconds) between haptic exploration of the reference orientation and matching of the test bar.

As expected, parallel matching induced large systematic errors which increased with distance. Overall error was significantly smaller in the mirror task. The task difference also held for the vertical orientation at $60 \mathrm{~cm}$ distance, even though this orientation required the same response in both tasks, showing a marked effect of task instruction. There was no significant change in performance for longer delay lengths. In addition, men outperformed women on the parallel task. Finally, contrary to our expectations, systematic errors were found in the mirror task, predominantly at $30 \mathrm{~cm}$ distance.

Based on these findings, we suggest that men are more apt at taking into account the orientation difference between the hands. In addition, we propose that haptic performance in near peripersonal space might be governed by different mechanisms than those which dominate at intermanual distances over $60 \mathrm{~cm}$. Short inter manual distances near the body correspond better to the dimensions of workspace for everyday manual tasks. At these distances, the orientation difference between the hands is relatively small, reducing the need to create an allocentric representation. The remaining errors might reflect the effect of the hand's rotational abilities and global perceptual factors.

Chapter 3 described the effect of adding visual information during the exploration phase on the orientation matching accuracy and time in haptic parallel and mirror tasks. Half of the participants used the right hand for exploration and the left for matching, and the other half vice versa. There was a fixed 5 second delay between exploration and matching, and visuo-haptic congruency was parametrically varied.

In line with our hypotheses we found that parallel matching parametrically varied with the level and direction of incongruent visual information. While visual information with an incongruence direction compatible with the direction of the egocentric bias decreased parallel matching accuracy, a moderate visual deviation in the opposite direction improved parallel matching accuracy. In contrast, mirror matching accuracy was unaffected in runs with incongruent visual information, while it was even reduced during runs with only congruent visual information. There was no general beneficial 
effect of visual information on parallel matching accuracy, neither for congruent visual runs, nor for incongruent visual runs.

These results clearly show that there is no effect of visual input per se, but that the effect of visual information on behavior depends on the type of haptic task, the stability of visuo-haptic correspondence and the visuo-haptic incongruency level. Moreover, it demonstrates that haptic tasks make flexible use of different strategies, either visual or somatosensory, with a specific susceptibility to multi-sensory integration.

In Chapter 4, female participants performed haptic parallel and mirror matching either with their body in a frontal position, or turned sideward, the legs at a 90 degree angle with the midsagittal plane of the head, which was maintained in a frontal position. Participants had six seconds to explore of the reference bar and simultaneously match its orientation by turning the test bar. In half of the trials, the left hand was used for exploration and the right hand for matching, and vice versa in the other half.

In line with our expectations, we found that mirror matching accuracy was reduced in the body sideward condition, while parallel matching was unaffected. In-depth analyses revealed that this effect was most pronounced in those participants who maintained their body in a leftward position, when they used their right hand for exploration of the reference bar. These results indicate that mirror matching performance, but not parallel matching performance, critically depends on body-centered representations.

\section{Tactile working memory for object orientation and surface roughness}

Whereas object orientation is important for grasp preparation, surface roughness is important for adequate scaling of grip force, given that a smooth object will slip from our hands when fingertip forces exceed surface friction. Different neural substrates have been shown to underlie the discrimination of macro spatial and micro spatial object features, such as object orientation and surface roughness. Tactile orientation discrimination was previously demonstrated to involve areas of the visual pathway, whereas roughness discriminations were shown to activate brain regions belonging to the tactile pathway. The studies described in chapters 5 and 6 of this thesis investigated which brain areas underlie tactile working memory, a process which maintains information online for upcoming tasks. Working memory for macro spatial bar orientations and micro spatial surface textures is important for manipulation and tactile recognition of objects. Right-handed participants were recruited for both studies.

Chapter 5 presented the imaging data obtained from 4 female and 3 male participants while they were performing a haptic parallel and mirror matching task inside the magnetic resonance scanner. They explored the orientation of the reference bar with the left hand and memorized its orientation. After a variable delay (0.5, 5 or 10 seconds), participants used their right hand to match the orientation by rotating another, identical (test) bar. No significant differences were found in the accuracy for parallel and mirror task, and event-related analysis of the BOLD response during exploration, delay and matching phase did not reveal any differences in the activation for parallel and mirror task.

In both parallel and mirror task, right sensorimotor cortex was active in the first seconds after the end of stimulus exploration, whereas clusters in left anterior prefrontal 
cortex (Brodmann area 10) became dominant two seconds after the end of exploration, showing sustained activity for several seconds. In contrast, left parieto-occipital cortex was involved at the end of the delay interval.

Our results indicate that the processing of haptico-spatial information in the delay between stimulus exploration and orientation matching is subserved by a dynamic network of brain areas. We propose that the contralateral somatosensory cortex supports the first tactile memory stage, as described by Burton and Sinclair (2000). This haptic sensory trace is transformed into a more abstract haptico-spatial representation early in the delay. The second memory stage might be reflected by the overlap of decreasing activation in primary somatosensory areas and upcoming activity in anterior prefrontal cortex. The third memory stage might engage anterior prefrontal cortex and parieto-occipital cortex. Anterior prefrontal cortex possibly integrates the spatial and motor components of haptico-spatial working memory, while parieto-occipital cortex might be involved in orientation imagery, supporting working memory and the preparation of haptic matching.

Chapter 6 describes the neural substrate for tactile texture working memory. Male participants performed a delayed match-to-sample task, in which 1, 2, 3 or 4 sandpaper stimuli had to be maintained in working memory during a 10 or $12 \mathrm{sec}$ delay interval. FMRI analyses revealed activation of primary and secondary somatosensory cortices during tactile encoding and matching, as well as delay-related activity in bilateral anterior inferior parietal lobule (aIPL), pre-supplementary motor area (pre-SMA) and right dorsolateral prefrontal cortex (dlPFC). Individual volume-of-interest analyses showed specific texture load dependent responses in right aIPL and right dlPFC, independent of the hand used for tactile encoding and matching. Left aIPL showed load-dependent responses and a texture preference for the right hand only. However, only on these right hand trials, did right aIPL show additional higher delay activation for accurate versus inaccurate trials. Pre-SMA neither revealed a higher response when the texture match-to-sample task was compared to a location control task, nor modulations for different texture loads.

Our results indicate a right-hemispheric dominance for tactile texture working memory. We propose that right dlPFC might subserve abstract cognitive monitoring processes and organization of multiple items in working memory. Right aIPL activation, on the other hand, might reflect domain-specific maintenance of tactile surface texture representations.

\section{Conclusions}

Chapters 2, 3 and 4 present the effects on haptic parallel and mirror matching of inter manual distance, gender, visual information, body posture and hand used for exploration and matching. We found differential effects for inter manual distance, body posture and visual information on parallel and mirror matching. Based on these findings, we conclude that haptic orientation matching might use a reference frame which is a weighted combination of body-centered and visual reference frames; a body-centered coding was dominant during mirror matching, whereas coding in a visual, eye- or headcentered frame was more important during parallel matching. 
Parallel matching showed dependence on inter manual distance and an error direction which corresponded to the natural orientation of the hand in space. This indicates that haptic spatial representations do not suffiently take into account the orientation of the hand in space. The fact that we found better parallel matching in males, suggests that they might be more skilled in accounting for hand orientation.

Parallel matching improved in the presence of visual information which subtly counteracted the ego-centric bias. This hand-dependent effect of visual incongruence direction, together with the pattern of errors in blindfolded parallel matching, demonstrates adding vision per se does not resolve the insuffiency in taking account of hand orientation in space. Mirror matching, in contrast, deteriorated in the presence of stable, congruent visual information. Under these circumstances, mirror matching might be forced to adopt a visual strategy, although detrimental to performance. However, the informational value of visual input decreases in a condition with varying visuo-haptic congruence. Therefore, incongruent information might have been ignored in the mirror task, which unlike the parallel task, can be performed with high accuracy using a body-centered representation alone.

Based on the results from the fMRI studies investigating the neural substrates of haptic working memory for macro spatial orientation and micro spatial surface texture, we conclude that haptic orientation working memory is subserved by a neural network including primary somatosensory cortex, anterior prefrontal cortex and parietooccipital cortex, while tactile texture working memory might be right-lateralized in men, supported by right inferior parietal cortex and right dorsolateral prefrontal cortex. Although obtained from different experiments, using different experimental designs and contrasts, our results are in accordance with the involvement of different neural substrates for the maintenance of micro spatial and macro spatial information, confirming the role of visual areas in the latter. Taken together, the fMRI results and the behavioral findings for haptic spatial matching might point to a specific role of the occipito-parietal areas in the representation of macro-spatial orientation, potentially in a visual, i.e. eye-centered or head-centered reference frame. On the other hand, right anterior inferior parietal lobe and right anterior prefrontal cortex are most likely of utmost importance for tactile microspatial working memory. 


\section{Samenvatting en Conclusies}

Dit proefschrift onderzocht de neurale basis van tactiele object manipulatie met behulp van gedragsmetingen en functionele magnetische resonantie (functional magnetic resonance imaging; fMRI), een niet-invasieve techniek die taakgerelateerde veranderingen in de hersenactiviteit in beeld brengt. Twee centrale onderzoeksvragen werden behandeld. Ten eerste bestudeerden we de referentiesystemen die gebruikt worden bij het uitvoeren van ruimtelijke taken met behulp van actieve tast (haptics). De resultaten van dit onderzoek werden gerapporteerd in hoofdstuk 2, 3 en 4 . Ten tweede maakten we gebruik van fMRI om te onderzoeken welke hersengebieden betrokken zijn bij het voelen en enkele seconden onthouden van klein- en grootschalige objectkenmerken, te weten oriëntatie (macrospatiëe) en ruwheid (microspatiëel). De bevindingen van deze fMRI experimenten werden gepresenteerd in hoofdstuk 5 en 6.

\section{Referentiesystemen in haptische ruimtelijke taken}

Een nauwkeurige voorstelling van de peripersoonlijke ruimte (de ruimte om ons lichaam) lijkt een noodzakelijke voorwaarde voor het succesvol hanteren van objecten. Desondanks werd gevonden dat haptische ruimtelijke voorstellingen behept zijn met systematische egocentrische fouten. Deze fouten zijn bijzonder opvallend in haptische oriëntatie-aanpassingstaken, waarin proefpersonen een teststaafje draaien dat de oriëntatie overeenkomt met die van een referentiestaafje, daarbij alleen gebruikmakend van actieve tast. In de paralleltaak is de instructie om het teststaafje naar een parallele oriëntatie $(/ /)$ te draaien. In de spiegeltaak daarentegen, wordt het teststaafje naar een orientatie gedraaid die het spiegelbeeld is van het referentiestaafje $(/ \backslash)$ in het midsagittale vlak van het lichaam. In beginsel vereist het correct parallel zetten van twee staafjes een allocentrisch referentiesysteem, onafhankelijk is van de lichaamspositie, waarin de ruimtelijke verhoudingen van de peripersoonlijke omgeving behouden blijven. De spiegeltaak, daarentegen, kan zowel in een ego- als een allocentrisch referentiesysteem nauwkeurig worden uitgevoerd. In een egocentrisch referentiesysteem is de ruimtelijke representatie gekoppeld aan het lichaam.

In eerdere studies zijn grote systematische fouten waargenomen in haptisch parallel zetten met $60 \mathrm{~cm}$ afstand tussen de handen; de grootte van de fouten en hun richting waren gecorreleerd met het verschil tussen de natuurlijke oriëntatie van de hand op de lokatie van de referentie- en teststaaf. In de spiegeltaak zijn echter bijna-waarachtige prestaties waargenomen. Op basis van deze resultaten is een gemeenschappelijk 
'tussenliggend' referentiesysteem voorgesteld voor de parallel- en spiegeltaak, opgebouwd uit een proefpersoon-afhankelijk gewogen gemiddelde van een egocentrisch, hand-gecentreerd systeem en een allocentrisch, extern-gebaseerd systeem. Aan de andere kant is ook gesuggereerd dat de paralleltaak het gebruik van een extrinsiek, visueel referentiesysteem bevordert, terwijl de haptische spiegeltaak een intrinsiek, handgecentreerd systeem bekrachtigt.

De experimenten beschreven in hoofdstuk 1, 2 en 3 zetten de verkenning voort van de eigenschappen van de referentiesystemen die haptisch parallel zetten en spiegelen domineren, door het effect van verschillende taakveranderingen op de nauwkeurigheid van rechtshandige proefpersonen te bestuderen. Met name het effect van de afstand tussen de handen, geslacht, visuele informatie, lichaamshouding en exploratie- en testhand werd bestudeerd, uitgaande van de rationale dat wanneer één van deze parameters de nauwkeurigheid van de oriëntatie-aanpassingen zou veranderen, dit een indicatie zou zijn dat het aan de taakuitvoering ten grondslag liggende referentiesysteem van deze parameter afhankeljk is. Alle proefpersonen waren rechtshandig. Tenzij anders vermeld, werd de referentiestaaf met de linkerhand gedurende 1,5 seconde verkend, en de teststaaf binnen 2 seconden met de rechterhand gedraaid. Referentie- en teststaaf waren op symmetrische posities geplaatst ten opzichte van het lichaamsmidsagittaalvlak, met een onderlinge afstand van $60 \mathrm{~cm}$.

Hoofdstuk 2 vergeleek de nauwkeurigheid van haptische oriëntatie-aanpassingstaken bij rechtshandige mannelijke en vrouwelijke proefpersonen, en rapporteerde de effecten veroorzaakt door het veranderen van de afstand tussen de handen $(30 \mathrm{~cm}$ of $60 \mathrm{~cm})$ en het tijdsinterval $(0,5,5$ of 10 seconden) tussen haptische exploratie van de referentieoriëntatie en draaien van de teststaaf.

Zoals verwacht leidde de paralleltaak tot grotere systematische fouten, die toenamen met afstand. Over het geheel genomen waren de fouten significant kleiner in de spiegeltaak. Het taakverschil hield ook stand bij de verticale orientatie op $60 \mathrm{~cm}$ afstand, ondanks het feit dat deze orientatie bij beide taken dezelfde respons vereist, waarmee een duidelijk taakeffect aangetoond werd. Er was geen significante prestatieverandering voor langere tijdsintervallen. Bovendien presteerden mannen beter dan vrouwen. Tot slot vonden we, in weerwil van onze verwachtingen, systematische fouten in de spiegeltaak, voornamelijk op $30 \mathrm{~cm}$ afstand.

Op basis van deze bevindingen concluderen we dat mannen beter in staat zijn rekening te houden met het oriëntatieverschil tussen de handen. Verder stellen we voor dat haptische taken die dichtbij het lichaam worden uitgevoerd mogelijkerwijs door andere mechanismen gedomineerd worden dan taken die worden uitgevoerd met een onderlinge afstand tussen de handen vanaf $60 \mathrm{~cm}$. Een situatie met de handen op korte onderlinge afstand dichtbij het lichaam komt beter overeen met de afmetingen van de werkruimte die bij alledaagse handelingen gehanteerd wordt. Op deze afstanden is het oriëntatieverschil tussen de handen relatief klein, waardoor er een beperkte noodzaak tot het vormen van een allocentrische voorstelling zou zijn. De resterende fouten zouden het draaivermogen en globale perceptuele factoren kunnen weerspiegelen. 
Hoofdstuk 3 beschreef het effect van visuele informatie tijdens de exploratiefase op de nauwkeurigheid en benodigde tijd voor de oriëntatie-aanpassing in de haptische parallel- en spiegeltaak. De helft van de proefpersonen gebruikten de rechterhand voor exploratie en de linkerhand voor het aanpassen van de teststaaforiëntatie, de andere helft vice versa. Er was een vast 5 seconden interval tussen exploratie en de oriëntatieaanpassing, en visuo-haptische congruentie werd parametrisch gevarieerd.

In overeenstemming met onze hypothesen vonden we dat nauwkeurigheid bij de paralleltaak parametrisch varieerde met de mate en richting van de incongruente visuele informatie. Terwijl de nauwkeurigheid in de paralleltaak verminderde bij visuele informatie met een incongruentierichting die overeenkwam met die van de egocentrische neiging, verbeterde de nauwkeurigheid juist door een gematigde visuele incongruentie in de tegengestelde richting. De nauwkeurigheid bij de spiegeltaak was daarentegen onveranderd in de reeks met incongruente informatie, terwijl zij zelfs verlaagd was in de reeks met enkel congruente informatie. Er was geen algemeen bevorderlijk effect van visuele informatie op de nauwkeurigheid in de paralleltaak, noch voor de congruente, noch voor incongruente experimentele reeks.

Deze resultaten tonen duidelijk dat er geen effect is van visuele informatie per se, maar dat het effect van visuele informatie afhankelijk is van het type haptische taak, de stabiliteit van de relatie tussen visuele en haptische informatie en de mate van visuohaptische incongruentie. Bovendien toont het aan dat er bij haptische taken flexibel gebruik gemaakt wordt van verschillende strategieën, visueel of somatosensorisch, met een specifieke gevoeligheid voor multisensorische integratie.

In Hoofdstuk 4 voerden vrouwelijke proefpersonen een haptische parallel- en spiegeltaak uit met hun lichaam in een frontale positie of gedraaid naar één kant, met de benen in een hoek van 90 graden ten opzichte van het midsagittale vlak van het hoofd, dat in een frontale positie gehouden werd. De proefpersonen hadden zes seconden om het referentiestaafje te verkennen en tegelijkertijd de oriëntatie van het teststaafje aan te passen. In de helft van de trials werd de linkerhand gebruikt voor exploratie en de rechterhand voor het draaien van de teststaaf, en vice versa in de andere helft.

Overeenkomstig met onze verwachtingen vonden we dat de nauwkeurigheid van de spiegeltaak verlaagd in de conditie waarin het lichaam gedraaid was, terwijl nauwkeurigheid bij de paralleltaak niet beïnvloed werd. Verdere analyses wezen uit dat dit effect het meest uitgesproken was in die proefpersonen waarbij het lichaam linkswaarts gedraaid was, en wanneer zij hun rechterhand gebruikten voor het verkennen van de referentiestaaf. Deze resultaten vormen een aanwijzing dat de spiegeltaak een kritische afhankelijkheid vertoont van lichaamsgecentreerde representaties.

\section{Tactiel werkgeheugen voor object-oriëntatie en ruwheid}

Terwijl object-oriëntatie belangrijk is voor het voorbereiden van een grijpbeweging, is ruwheid belangrijk voor een gepaste afstemming van de grijpkracht, gegeven het feit dat een glad object uit onze handen zal glippen als de kracht van onze vingertoppen de wrijving van het oppervlak overschrijdt. Van verschillende neurale gebiede is aangetoond dat ze bijdragen aan het onderscheiden van macro- en microspatiële objectkenmerken, zoals oriëntaie en ruwheid. Het op de tast onderscheiden van oriëntatie doet een beroep op gebieden van de visuele verwerkingsroute, terwijl het onderscheiden van 
ruwheid hersengebieden activeert van de tactiele verwerkingsroute. De studies beschreven in hoofdstuk 5 en 6 van dit proefschrift onderzochten welke hersengebieden ten grondslag liggen aan tactiel werkgeheugen, een process dat informatie toegankelijk houdt voor aanstaande taken. Werkgeheugen voor macrospatiële staaforiëntaties en microspatiële oppervlakteruwheid is belangrijk voor het hanteren en op de tast herkennen van objecten. Voor beide studies werden rechtshandige proefpersonen gerecruteerd.

Hoofdstuk 5 presenteert de hersenactiviteit van 4 vrouwelijke en 3 mannelijke proefpersonen die gemeten werd terwijl zij een haptische parallel- en spiegeltaak in de magnetische resonantie scanner. Zij verkenden de oriëntatie van het referentiestaafje met de linkerhand en memoriseerden de oriëntatie. $\mathrm{Na}$ een variabel tijdsinterval $(0,5,5$ of 10 seconden) gebruikten de proefpersonen hun rechterhand om de orientatie van een ander identiek (test)staafje aan die van het eerste staafje aan te passen. Er werden geen significante verschillen gevonden tussen de nauwkeurigheid in de parallel- en de spiegeltaak.

Onze resultaten geven aan dat de verwerking van de haptico-spatiële informatie in het tijdsinterval tussen stimulusverkenning en het aanpassen van de oriëntatie ondersteund wordt door een dynamisch netwerk van hersengebieden. We stellen voor dat de contralaterale somatosensorische cortex betrokken zijn bij het eerste stadium van tactiel geheugen, zoals beschreven door Burton and Sinclair (2000). Deze sensorische geheugenindruk wordt vroeg in het geheugeninterval omgevormd tot een meer abstracte haptisch-ruimtelijke voorstelling. Het tweede geheugenstadium zou gevormd kunnen worden door de overlap van afnemende activatie in de primaire somatosensorische gebieden, en opkomende activiteit in de anterieure prefrontaalcortex (aPFC). Het derde geheugenstadium zou de anterieure prefrontaalcortex en de parietaaloccipitaalcortex kunnen activeren. Het is mogelijk dat de anterieure prefrontaalcortex de spatiële en motorische componenten in het haptisch-ruimtelijk werkgeheugen integreert, terwijl de parietaal-occipitaalcortex betrokken zou kunnen zijn bij het zich visuele voorstellen van de oriëntatie, ter ondersteuning van het werkgeheugen en de voorbereiding van de haptische oriëntatie-aanpassing.

Hoofdstuk 6 beschrijft de neurale grondslagen van tactiel geheugen voor weefselstructuur in de vorm van ruwheid. Mannelijke proefpersonen voerden een uitgestelde vergelijkingstaak uit, waarin kenmerken van 1, 2, 3, of 4 stukjes schuurpapier gedurende een interval van 10 of 12 seconden in het werkgeheugen gehouden moesten worden.

De analyse van functionele magnetische resonatiebeelden onthulde activiteit in de primaire en secundaire somatosensorische cortex tijdens het voelen en vergelijken van de stukjes schuurpapier, en activiteit gerelateerd aan het geheugeninterval in de linker en rechter anterieure inferieure parietaalkwab (aIPL), pre-supplementaire motorische gebieden (pre-SMA) en de rechter dorsolaterale prefrontaalcortex (dlPFC). Analyse van individueel gedefiniëerde interessegebieden toonden aan dat de activiteit in de rechter anterieure inferieure parietaalkwab en de rechter dorsolaterale prefrontaalcortex specifiek was voor het onthouden van ruwheid en toenam met het aantal ruwheden, onafhankelijk welke hand gebruikt werd voor het voelen en het vergelijken. De linker anterieure inferieure parietaalkwab liet alleen bij gebruik van de rechterhand een hogere 
activiteit voor ruwheid en een aantal-afhankelijkheid zien. Daarbij vertoonde de rechter anterieure inferieure parietaalkwab alleen bij het gebruik van de rechterhand voorafgaand aan correcte antwoorden

hogere activiteit dan voorafgaand aan incorrecte antwoorden. De pre-supplementaire motorische gebieden openbaarden geen hogere respons voor de ruwheidvergelijkingstaak vergeleken met een lokatievergelijkings-controletaak, noch lieten ze een modulatie zien door het aantal te onthouden ruwheden.

Onze resultaten duiden erop dat de rechter hersenhelft dominant is bij tactiel werkgeheugen voor ruwheid. We stellen voor dat de rechter dorsolaterale prefrontaalcortex een rol speelt bij abstracte cognitieve controleprocessen en het organiseren van meervoudige items in het werkgeheugen. Aan de andere kant zou de activatie van de rechter anterieure inferieure parietaalkwab de handhaving van een domein-specifieke voorstelling van tactiele weefselstructuur kunnen weerspiegelen.

\section{Conclusies}

Hoofdstuk 2, 3 en 4 presenteren de effecten van afstand tussen de handen, geslacht, visuele informatie, lichaamshouding en de hand gebruikt voor verkenning en aanpassing van de staaforiëntatie op een haptische parallel- en spiegeltaak. We vonden disctinctieve effecten voor handafstand, lichaamshouding en visuele informatie op de parallel- en de spiegeltaak. Op basis van deze bevindingen concluderen we dat haptische oriëntatie-aanpassingstaken mogelijk een referentiesysteem gebruiken dat een gewogen combinatie is van lichaams-gecentreerde en visuele referentiesystemen; lichaamsgecentreerde codering had de overhand tijdens de spiegeltak, terwijl codering in een visueel oog- of hoofd gecentreerd referentiesysteem van groter belang was tijdens de paralleltaak.

De paralleltaak vertoonde een afhankelijkheid van de afstand tussen de handen en een foutenrichting die overeenkwam met de natuurlijke oriëntatie van de hand in de ruimte. Dit duidt erop dat haptiscje ruimtelijke voorstellingen niet genoeg rekening houden met de ruimtelijke orientatie van de hand. Het feit dat mannelijke proefpersonen beter presteerden op de paralleltaak suggereert dat zij beter in staat zijn om met handoriëntatie rekening te houden.

De prestaties op de paralleltaak verbeterden in de aanwezigheid van visuele informatie die op subtiele wijze tegen de egocentrische richtingsafwijking inging. Dit handafhankelijke effect van de visuele incongruentierichting, samen met het foutenpatroon bij het geblinddoekt uitvoeren van de paralleltaak, tonen aan dat het toevoegen van visuele informatie per se niet genoeg is om de onvolkomendheid in het meeberekenen van handoriëntatie te compenseren. Presetaties on de spiegeltaak verslechterden daarentegen in de aanwezigheid van stabiele, congruente visuele informatie. Het is mogelijk dat proefpersonen onder deze omstandigheden gedwongen zijn om ook voor de spiegeltaak een puur visuele strategie te kiezen, hoewel deze de prestaties negatief beïnvloedt. De informatieve waarde van visuele input vermindert daarentegen in een conditie met wisselende visuo-haptische congruentie. Om die reden zou incongruente informatie genegeerd kunnen worden in de spiegeltaak, die in tegenstelling tot de paralletaak op basis van enkel een lichaamsgecentreerde voorstelling met grote nauwkeurigheid kan worden uitgevoerd. 
Op basis van de resultaten van de functionale magnetische resonantie-studies die de neurale grondslagen van haptisch werkgeheugen voor macrospatiële oriëntatie en microspatiële ruwheid, concluderen we dat haptisch werkgeheugen voor oriëntatie ondersteund wordt door een neuraal netwerk bestaande uit de primaire somatosensorische cortex, anterieure prefrontaalcortex en de parietaal-occipitaalcortex, terwijl bij tactiel werkgeheugen voor ruwheid de rechter hersenhelft, en met name de rechter inferieure anterieure parietaalkwab en de rechter dorsolaterale prefrontaalcortex een belangrijke rol spelen (in mannen). Hoewel deze resultaten in afzonderlijke experimenten verkregen werden, met verschillende experimentele opzet en statistische contrasten, zijn ze in overeenstemming met de betrokkenheid van verschillende neurale gebieden bij het vasthouden van micro- en macrospatiële informatie, en bevestigen ze de rol van visuele gebieden met betrekking tot de laatstgenoemde objectkenmerken. Samengenomen duiden de resultaten verkregen met functionele magnetische resonantie en de gedragsresultaten voor haptisch oriëntatie-aanpassingstaken erop dat de occipito-parietale gebieden een specifieke rol zouden kunnen spelen bij de representatie van macrospatiele oriëntatie, mogelijk in een visueel, oog- of hoofd-gecentreerd referentiesysteem. Aan de andere kant zijn bij tactiel werkgeheugen voor microspatiële objectkenmerken waarschijnlijk juist gebieden in de rechter anterieure inferieure pariëtaalkwab en de rechter anterieure prefrontaalcortex van het grootste belang. 


\section{Epiloog}

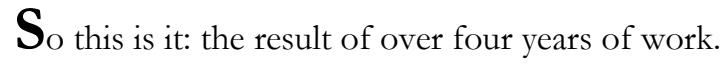

I am very proud that I finished it. It took great effort, more than I ever put into any project before in my life, shaping me both as a researcher and as a person. I have learned so much on the way. I am sure that this learning can be a fertile basis for many more scientific and other projects in the coming years.

Een kleine tien jaar geleden, toen ik nog niets van universiteiten of wetenschap wist, was het woord 'promotie' voor mij geassocieerd met bevorderd worden naar een meer verantwoordelijke functie door een hogergeplaatste. Een tamelijk passief gebeuren dus, ook al vindt het zijn basis in getoonde kwaliteiten en vermoede talenten. Maar bij een promotie in academische zin gaat het om de actieve betekenis van het woord: voordat de dag van je promotie aanbreekt heb je als het goed is zelf een beweging naar voren doorgemaakt, op wetenschappelijk, maar ook op persoonlijk vlak. De voorwaartse beweging die ik in de afgelopen jaren heb gemaakt had niet kunnen plaatsvinden zonder de hulp van vele mensen om mij heen...

Hanneke van Mier, my most reliable and fervent supporter at the UM, your meticulous reading guarded me from over-spontaneous submissions and helped me get over my aversion of proofreading my own manuscripts. I learned that finishing a paper is like biking to Friesland across the Afsluitdijk: you already see where you will be going after the first curve, but then it still takes a lot of patient pedaling against the wind to get to the other side... you insisted that I'd continue pedaling, and I discovered the value of going all the way.

Rainer Goebel, your enthusiasm for neuroscience is absolutely contagious and attracts people to Maastricht from all over the world. You have a heart for science and for people, which makes it a joy to be part of your group and your world.

Big thanks to the people of BrainInnovation, who were always prepared to repeat Rainer's fast-forward clicking through Brainvoyager in slomo. Thanks, Armin, for your invaluable statistical support, and Hester for being so attentive. Henk, it was nice meet- 
ing someone with a similar weakness for Germans and music. Thanks for the guitar lessons and the technical support both in- and outside of the university building.

De vreemdsoortige relikwieën in mijn kamer op de UNS40 zijn de stille getuigen van de hulp van de mensen van de technische dienst bij het realiseren van mijn ietwat ongebruikelijke stimulusmateriaal. Johan Gielissen, Ron Hellenbrand, Jacco Ronner en Huub Hamers, bedankt! Sven Gijsen, Erik van den Bergh en Paul Gaalman (F.C. Donders centrum), bedankt voor de hulp bij het scannen.

Alle studenten die met mij hebben samengewerkt, speciaal Maya Visser, Johan Lataster en Nicolette Siep: bedankt voor jullie inzet! Naast het feit dat jullie de dataverzameling een stuk lichter gemaakt hebben, waren jullie vragen en interesse een bron van inspiratie.

Elia Formisano, your preparedness to sit down with desperate fMRI-newbies and look at their raw data are unequalled, just like your pasta. I hope I will have the opportunity to work with you and learn even more from you in the future. Peter de Weerd, mede Brel-fan, jouw nauwkeurige wetenschappelijke manier van redeneren, je oprechtheid en pianistische vaardigheden zijn bewonderenswaardig. Hartelijk dank voor de tijd die je geïnvesteerd hebt in het geven van gedetailleerde feedback op mijn manuscript. Bernadette Jansma, bedankt dat je oog en oor hebt voor je medewerkers en echt je best voor ze doet. Annemie Jeukens, bedankt voor al je goede zorgen, speciaal in de laatste fase toen ik al in Frankfurt aan de slag was maar mijn manuscript nog ingebonden en opgestuurd moest worden aan de leescommissie.

Joel Reithler, het spreekwoord stille wateren hebben diepe gronden moet wel voor jou bedacht zijn... dankjewel voor je mentale en inhoudelijke steun de afgelopen jaren. Ik bewonder je precisie, en wenste me dat ik zo geduldig kon zijn als jij. Ik blijf nieuwsgierig naar je composities...

Alard, Anke, Anton, Bettina, Erik, Federico, Francesco, Giancarlo, Hanna, Holger, Ingrid, Jessica, Judith, Leo, Lisa, Lars, Lourens, Marjo, Mart, Niko, Noel, Riny, Teresa, Vera, Wijnand, Wilfred en alle andere mensen die de afdeling Neurocognitie en de faculteit Psychologie bevolkten in de afgelopen jaren, bedankt voor de positieve werksfeer, de bereidheid om te helpen, het kletsen in de koffiehoek en de feestjes. Michael, ex-buurman, jij bent met afstand de beste man om mee te koffieleuten. Vincent, ik hoop op nog vele gitaarpauzes in de toekomst.

Milene, Nienke, Sandra en Fraukje, jullie verdienen een eigen alinea. Collega's maar vooral vriendinnen om mee te praten, te lachen, te drinken, te dansen... Ik hoop dat we met onze weekendjes weg een traditie hebben gezet. Sandra, die halve marathon hebben we niet gered, maar met die promoties komt het helemaal goed! Fraukje, heerlijk om je te horen lachen en met jou te genieten van absurde situaties.

Lieve paranimfen: de driehoek is rond! Milene, wat ben ik blij dat jij (mét Rachmaninov) mijn kamergenoot was. Dat waren flinke golven de afgelopen maanden... maar we zijn er nog, en hoe. Jouw felheid en sociale zintuig, die ondeugende blik in je ogen als je weer iets op het spoor bent, zijn een onweerstaanbare combinatie. Nienke, exhuisgenoot, doelgericht en verstandig, maar wel met een wijntje! Ik bewonder je standvastigheid, en leerde van jou dat geluk ook een keuze is. Dank voor je gastvrijheid, de weekendjes Texel. Wanneer organiseren we weer eens een feestje? 
Thanks also to Sarah Weigelt, Lars Muckli and my other new colleagues at the Max Planck Institute and Brain Imaging Centre in Frankfurt, for their support during the last months. Franca Symalla, Danke für Deine Hilfe mit den Referenzen.

Mijn NWO projectgroep, in het bijzonder Astrid Kappers, Ruud Meulenbroek, Mary Klein-Breteler, bedankt voor het meedenken. Sander Zuidhoek, het is altijd weer leuk jou tegen te komen en te discussiëren over (haptische) experimenten en andere zaken. Ester Reijnen, jouw aanwezigheid in Maastrich maakte het werken aan mijn eerste fMRI experiment een stuk leuker. Cornelia Stoeckel, the first person I met at an fMRI conference with a similar interest in the tactile system. Thanks for inviting me to Düsseldorf, maybe that joint tactile project will come one day... Jody Culham, thanks for accepting the invitation to give a talk at the Object Manipulation symposium in Nijmegen. It was great talking to you and meeting the woman behind the fMRI for Dummies - oops - Newbies website.

In Maastricht kon ik ook mijn onwetenschappelijke kanten uitleven. Johan van Amersfoort, de mensen van mijn dramagroep: het was heerlijk om bij jullie de creativiteit te voelen borrelen en aan den lijve te ondervinden dat communicatie eigenlijk maar weinig met woorden te maken heeft. En Johan, de belangrijkste ontwikkelingen worden inderdaad door kritiek en weerstand in gang gezet. Lucas Blommers, het streven naar perfectie remde gelukkig niet tijdens die tweewekelijkse zanglessen ... Dank ook aan de mensen van Lingua e Musica, Jo, Tineke, Carla, Rozemarie, voor de fijne donderdagen andere avonden, de betoverende werking van het zingen in de Cellebroederskapel.

Katinka, Gaby en Nolda, dank voor de etentjes; Nolda ook voor Tommie die helaas jammerlijk in een modderstroom ten onder is gegaan en niet te vergeten voor de sleutel van je huis. 'Tricia en Niels, dank voor het regelmatige note-bashing, jullie interesse in mijn werk, en de leuke discussies over van alles en nog wat; Niels, ik blijf genieten van jouw chocolademousse en de originele manier waarop je de mensen om je heen laat weten dat ze speciaal zijn.

Jorg - voor al uw problemen en voor een ander perspectief - fijn dat we onze dramatische persoonlijkheidsstroornis tot muziek kunnen sublimeren.

In de verte maar toch dichtbij waren Lody, Karin en Willemijn. Ik ben blij dat we elkaar niet uit het oog verloren zijn, ondanks die autistische periode in het laatste jaar van mijn promotie...

Danielle Tisserand, we ontmoetten elkaar op mijn eerste werkdag, ik heb genoten van onze wetenschappelijke discussies om 8 uur 's ochtends tijdens het hardlopen, en later van mijn bezoekjes aan jou in Canada, en de reis met Anne en Pascal. Mijn meubilair in Frankfurt stamt voor een groot deel uit jouw huis op het Dopplerdomein.

Marieke van Asselen, ik heb veel gehad aan jouw nuchtere kijk op wetenschap en promoveren. Die berg in Bolivia was één van de meest intense ervaringen in mijn leven. Ik verheug me op nog veel meer bezoekjes aan Lousa en Coimbra...

Alex, Dein Vertrauen hat mir unglaubliche Stärke gegeben. You taught me to never give up, never to stop believing that everything will be alright in the end. Your prag148 
matic approach to life softened my fastidious nature, focused my uncoordinated stream of ideas and canalized my Brownian motion through (academic) space. Zonder jou waren de afgelopen twee jaar zoveel zwaarder geweest, had ik nooit deze eindsprint kunnen maken.

Bernard, Ik ben heel blij dat ik zo'n fijne broer heb. Ik geniet ervan om je over je werk te horen praten, en mijn ideeën te testen naar jouw natuurkundige maatstaven. Hopelijk vergeef je me dat ik - ondanks jouw verwoede anti-campagne - nog steeds Microsoft-gebruiker ben en mijn proefschrift in Word en niet in LaTeX is gemaakt. Doen we nog een keer een weekje Alpen als jij klaar bent met jouw proefschrift?

Pap en mam, zelf al meer dan dertig jaar op hetzelfde adres hielpen jullie mij ontelbare keren verhuizen, en kwamen graag op bezoek in weer een nieuwe stad. Jullie hebben me altijd gestimuleerd en mijn keuzes nooit direct proberen te beïnvloeden. Doen wat je leuk vindt was het devies, en of dat nu betekende een jaar naar Mexico of psychologie gaan studeren terwijl leraren me dat sterk afraadden, jullie accepteerden het zonder blikken of blozen. En met de jaren merk ik steeds meer dat motivatie echt zwaarder weegt dan talent.

Oma, als kind kwam ik elke zaterdagochtend bij u theedrinken en vertelde u me over vroeger, hoe jammer $\mathrm{u}$ het vond dat $\mathrm{u}$ niet verder kon leren na de lagere school. $\mathrm{U}$ te zien glimmen van trots toen ik vertelde dat ik van plan was mijn proefschrift aan $u$ op te dragen was een heel bijzonder moment. 


\title{
Curriculum vitae
}

\begin{abstract}
Amanda Kaas was born on October 9, 1975 in Alkmaar, The Netherlands. In 1994 she completed her secondary school education at the Murmellius Gymnasium in Alkmaar, after which she spent a year in Mexico as an exchange student. In 1995 she started her Psychology study at Leiden University. In 1996 she continued her studies at the University of Amsterdam (UvA), where she completed a minor in Mathematics between 1999 and 2001. After internships at the department for developmental psychology, the methodological student advisory board at the UvA and the MEG (magneto-encephalography) center of the academic hospital of the Free University in Amsterdam, she obtained her Master's degree in Psychonomics specializing in quantitative psychological research. In December 2001, she started her Ph.D. research at the Department of Cognitive neuroscience at Maastricht University. She is currently working as a postdoc at the department of Neurophysiology of the Max Planck institute for Brain Research in Frankfurt am Main, Germany.
\end{abstract}

Amanda Kaas is op 9 oktober 1975 in Alkmaar geboren. In 1994 behaalde zij haar VWO diploma aan het Murmellius Gymnasium in Alkmaar, waarna zij een jaar als uitwisselingsstudent naar Mexico verbleef. In 1995 begon ze haar studie Psychologie aan de Rijksuniversiteit Leiden. $\mathrm{Na}$ het behalen van haar propedeuse zette ze haar studie in 1996 voort aan de Universiteit van Amsterdam (UvA), alwaar ze in de periode 1999-2001 de pas opgezette minor Wiskunde voltooide. Na stages bij de afdeling Onwikkelingspsychologie, de Methodenwinkel (een statistisch en methodologisch adviesburo van de UvA), en het MEG (Magneto-encepohalografisch) centrum van het academisch ziekenhuis van de Vrije Universiteit in Amsterdam, studeerde zij in februari 2001 af in de richting Psychonomie met als studieroute kwantitatief psychologisch onderzoek. Zij begon haar promotieonderzoek aan de afdeling Neurocognitie van de Universiteit Maastricht in december 2001. Op dit moment is zij werkzaam als postdoc bij de afdeling Neurofysiologie van het Max Planck Instituut voor Hersenonderzoek in Frankfurt am Main, Duitsland. 


\section{Appendix}

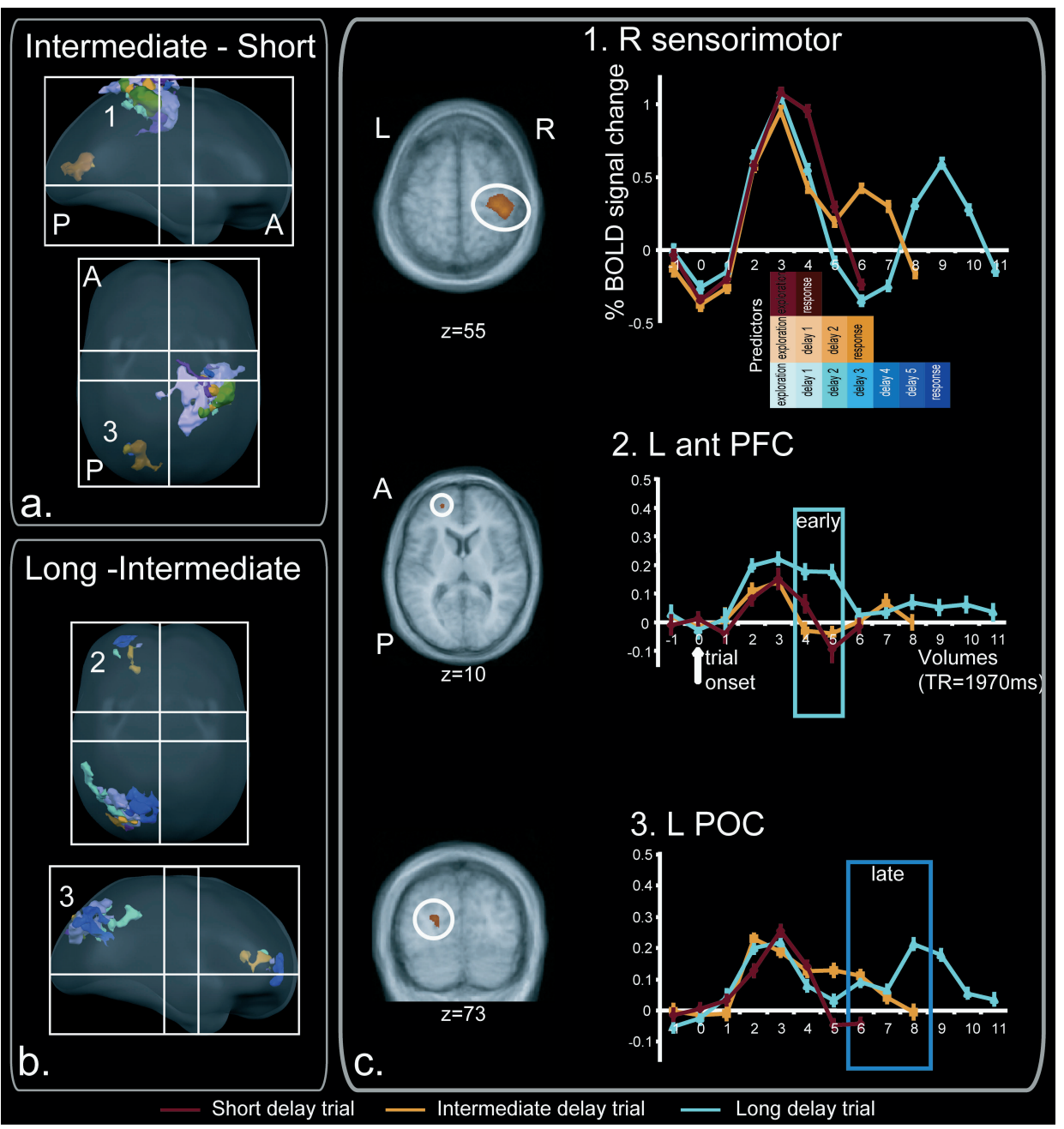

Figure 5. (chapter 5) Selected delay-length dependent areas from single-subject (a. and b.) and group analyses (c.). Individual regions of interest for the contrast of intermediate minus short trials (a.) and long minus intermediate trials (b.) are projected in a glass brain (right and top views). 1 = right sensorimotor cortex; 2 $=$ left anterior prefrontal cortex (aPFC); $3=$ left parieto-occipital cortex (POC). Clusters c.: The right sensorimotor cluster was obtained from the contrast of intermediate minus short delay trials (group: $t>2.9$, $\mathrm{q}(\mathrm{FDR})<0.05$; single-subject: $\mathrm{t}>2, \mathrm{p}<0.05)$, and the left aPFC cluster from the contrast of long minus intermediate trials (group: $\mathrm{t}>3.3, \mathrm{q}(\mathrm{FDR})<0.05$; single-subject: $\mathrm{t}>2, \mathrm{p}<0.05$ ). A similar left POC cluster was found in both contrast analyses; displayed is the time course from the cluster found by subtracting short from intermediate trials. A square window indicates the time points of interest for the left aPFC and left POC. The group clusters were projected on the group average of the Talairach normalized brains. Note that the slight spilling of the clusters from the glass brain surface is due to the fact that the surface does not reach until the superior end of Talairach space. 


\section{WM related activity}

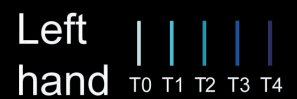

Right

A.

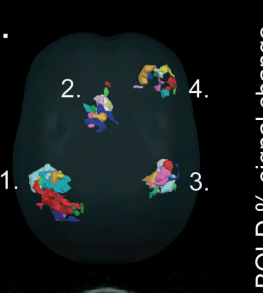

B.

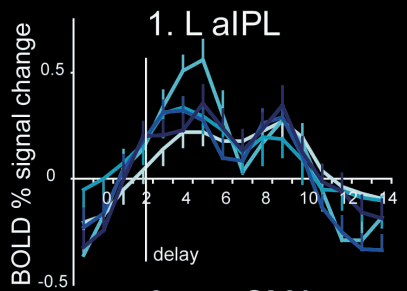

2. pre SMA

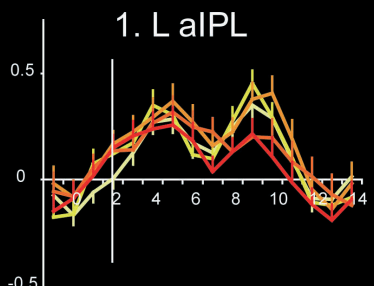

2. pre SMA
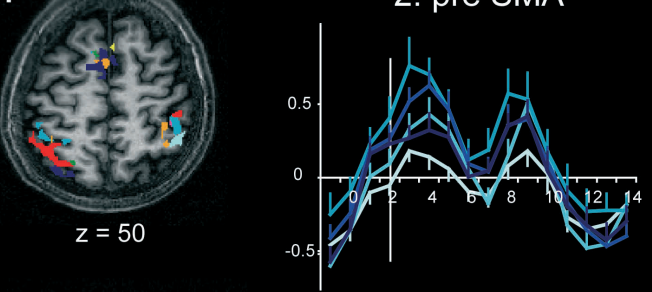

3. R alPL

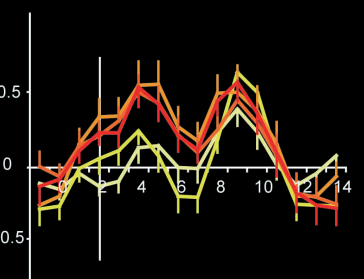

C.
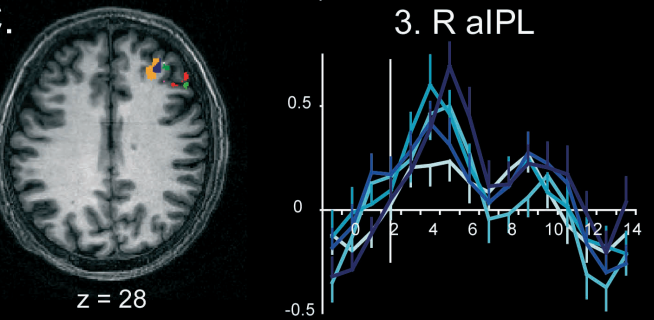

3. $\mathrm{R}$ alPL

D.

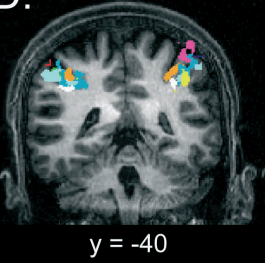

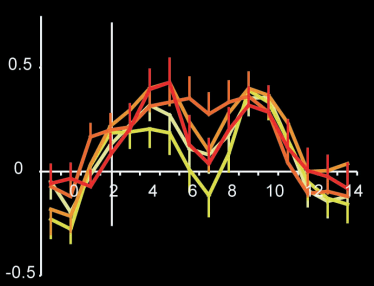

4. R dIPFC
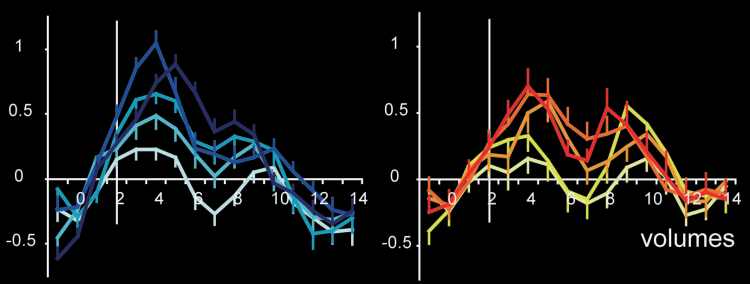

Figure 4. (chapter 6) Event-related time courses for each cluster activated in the texture delay, revealed by the group fixed-effects GLM. The trial starts at volume zero, and the start of the delay phase is indicated by a white vertical bar (at volume=2). a: A glass brain, viewed from above, with the group delay clusters displayed in white, as well as the individual VOIs for texture delay load 1, 2, 3 and 4 versus inter trial interval. Each color represents a single participant. 1= left anterior inferior parietal lobule (L aIPL); $2=$ presupplementary motor area (pre SMA); $3=$ right anterior inferior parietal lobule (R aIPL); 4= right dorsolateral prefrontal cortex (R dlPFC). b, c and d: the individual VOIs projected on different slices of a participant's brain. Left in the picture corresponds to left in the brain. 